LBL -31639

DE92 017105

\title{
PREPARATION AND CHARACTERIZATION OF LOW-DEFECT SURFACES
}

\author{
TIMOTEY OREN ROBINSON \\ Ph.D. Thesis \\ COJIEGE OF CHEMISTRY \\ University of California \\ and \\ MATERIAIS SCIENCE DIVISION \\ Iawrence Berkeley Laboratory \\ University of California \\ Berkeley, CA 94720 \\ DECEMBER， 1991
}

This work was suppoxted by the Director, Office of Energy Research, office of Raslc Energy sciencer, Materials Sciencen Diviaion, of the U.S. Department of Energy under Cantract. Wo. DE-AC03-765F00098 


\title{
Preparation and Characterization of Low-Defect Surfaces
}

\author{
Copyright @ 1991
}

by

Timothy Oren Robinson

The U.S. Department of Energy has the right to u.se this thesis for any purpose whatsoever including the right to reproduce all or any part thereof 
Preparation and Characterization of Low-Defect Surfaced

by

Timothy Oren Robinson

Abstract

silver cryatal surfaces with low defect densities were prepared electrochemically from queous solutions using capillary-growth techniques. These aurfaces had low rates for the nucleation of new silver layers. The impedance of these inert silver/aqueous bilver nitrate interfaces was used to determine silver adatom concentration and water dipole reorientation energetics. At room temperature, the normsized silver adaton arface coverage is low, $\theta=2.7 \times 10^{-3}$, and

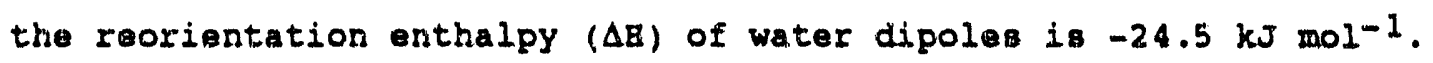
Growth of silver crystals that contained redox-inactive, nucleation enhancing defects resulted in unusual temporally-deterministic monolayer growth events. Study of the waiting time between these growth events allow determination of surface diffusion coeficients for silver adatoms. We calculate $D_{B}=(1.2 \pm 0.3) \times 10^{-7} \mathrm{~cm}^{2} \mathrm{~s}^{-1}$ at $\mathrm{T}=20^{\circ} \mathrm{C}$, and $D_{g}=(2.5 \pm 0.9) \times 10^{-7} \mathrm{~cm}^{2}$ at $\mathrm{T}=50^{\circ} \mathrm{C}$.

Computational techniques were applied to the determination of time-dependent microscopic growth rates and to the simulation of diffusive mass transport at low concentrations in a chemical potential gradient. Monolayer growth resulting from expansion of a circular growth front into an elliptical boundary was analyzed and algorithmo were developed for converting experimental observables into the initial location of the nucleation site. For a boundary with minor axis length 
$77 \mu m$ and an eccentricity of 0.65 , the methods determine the nucleation site to within a mean deviation of $540 \mathrm{~nm}$ from the actual site. The mass transport simulatione were performed on a finite ono-dimensional lattice and the resulte were extended to adatom trangport across macroscopic surfaces.

Techniques and instrumentation for acanning tunneling microscopy and pectroscopy were developed. Simultaneously acquired topography and differential conductance data were visualized by texture-mapping colorcoded conductance data onto a three-dimeneional representation of the topography. This visualization technique allowa correlation of electronically unique surface features with local structure. For example, terrace region on polycrystalline platinum surfaces denonstrated work function differences on the oxdar of $1 \mathrm{eV}$. Steps on highly oriented pyrolytic graphite (BOPG) were observed to have significantly lower work function than surrounding terrace regions by 1 $\pm 0.5 \mathrm{eV}$. Faulted and unfaulted surface layors of BOPG were found to exhibit different differential conductance, consistent with all work function variations between the surface structures. 
DEDICATION

This thesis is dedicated to the memory of my mother,

Eelen Louise Robinson 


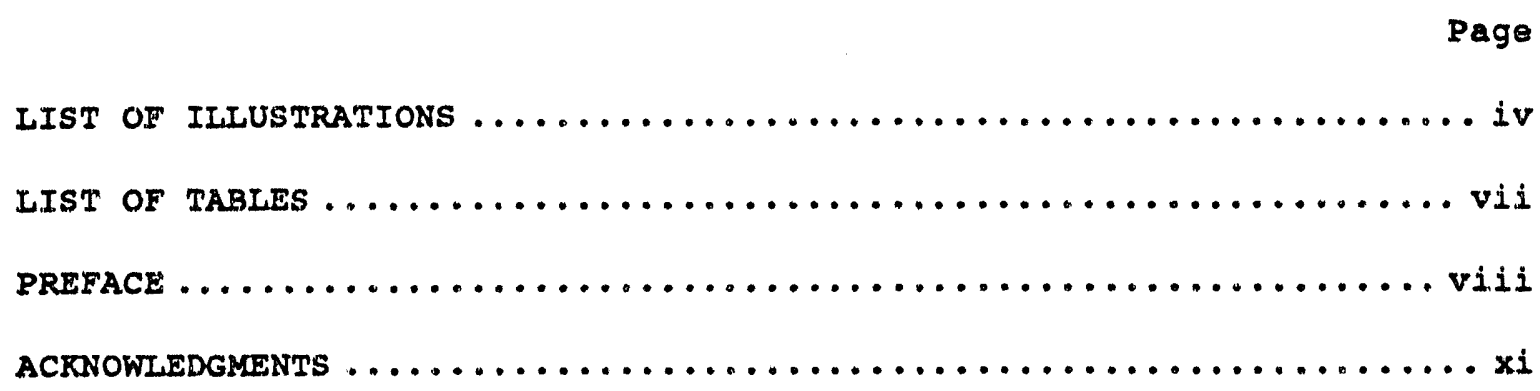

1. PREPARATION AND CBARACTERIZATION OF LOW DEFECT SILVER CRYSTAL

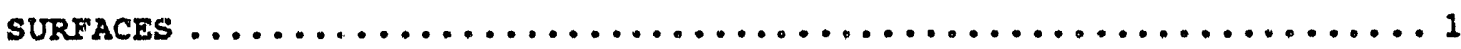

2. COMPUTATIONAL MODEIING OF GROWTE IN SIMPLE SYSTEMS ........... 70

3. SCANNING TUNNELING MICROSCOPY AND SPECTROSCOPY OF REALISTIC

SURFACES ........................................ 117

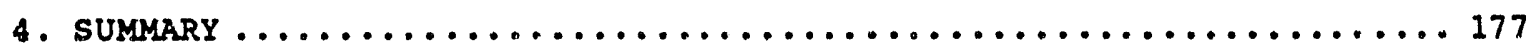

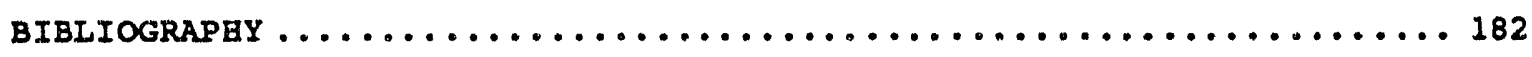

APPENDIX A.......................................... 189

APPENDIX B......................................... 223

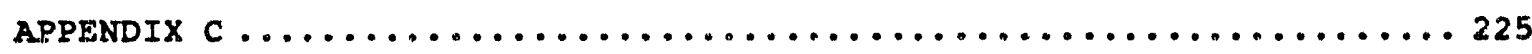

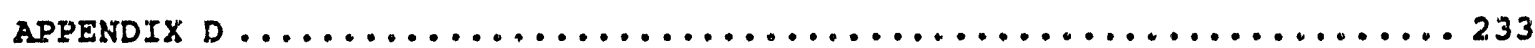


1. Control signal generation circuit ..................51

2. Two buffer circuits ............................52

3. Throe-electrode potentiostat. .....................53

4. Two-electrode cell control circuit ...................54

5. sketch of experimental cell ..................... 55

6. Preparation of short capiljaries ....................56

7. Thermal control configurations ....................57

8. Oscilloscope traces during growth ................... 58

9. Geometry of active portion of aurface .................59

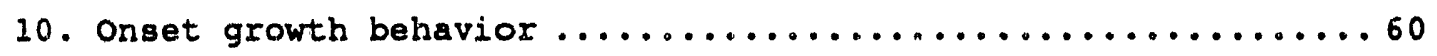

11. Nucleation rate veraus overpotential ................61

12. Admittance of inert uurface ....................... 62

13. Interfacial dielectric constant vs. $1 / \mathrm{T} \ldots \ldots \ldots \ldots \ldots \ldots$

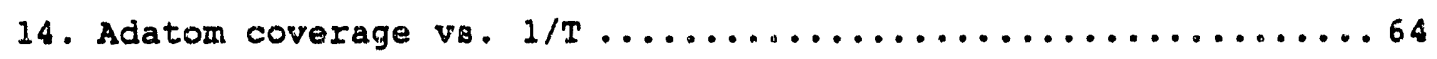

15. single growth event $\ldots \ldots \ldots \ldots \ldots \ldots \ldots \ldots \ldots \ldots \ldots \ldots \ldots \ldots \ldots \ldots \ldots \ldots \ldots \ldots \ldots$

16. Long time series of growth ......................66

17. sketch of active surface in capillary ...............67

18. Subset of data in illustration $16 \ldots \ldots \ldots \ldots \ldots$

19. Microfacet size Vo. square root of nucleation waiting time..69

20. Examplen of symetry-equivalent sites ............... 103

21. critical points in a growth event .................. 104

22. Three simulated growth events .................... 105

23. Two growth events within an elliptical boundary ........ 106 


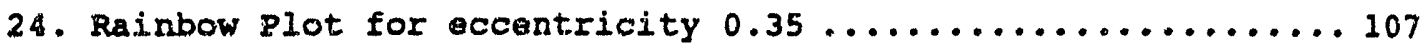

25. Intermediate construction illustration $\ldots \ldots \ldots \ldots \ldots \ldots \ldots$

26. Comparison of initial and calculated nucleation sitas

$e=0.35$

27. Comparison of initial and calculated nucleation sites

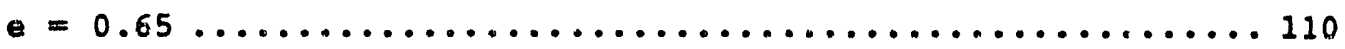

28. Boundary and terms in experimental modeling calculations ... 111

29. Model curves for different eccentricities of boundary ..... 112

30. Model curves for different nucleation site locations ...... 113

31. Impact on uncertainty in d on position determination ....... 114

32. Methodology of surface diffusion simulations ............ 115

33. Regults from a set of diffusion simulations $\ldots \ldots \ldots \ldots \ldots \ldots 116$

34. Junction electronic band structure ............... 152

35. Physical mount for microscope apparatus $\ldots \ldots \ldots \ldots \ldots \ldots \ldots$

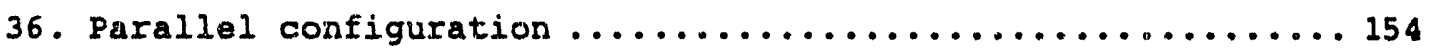

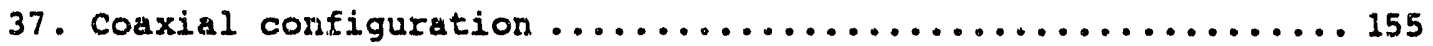

38. Configuration of piezoelectric Glements in scanning head ... 156

39. Line representation of a gold surface topograph ......... 157

40. Sketch of conductance measurements ................. 158

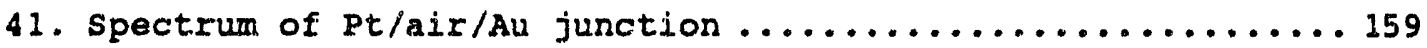

42. Spectrum of Pt/air/Cds/Au junction .................. 160

43. Bopg topography and conductance linescans ................161

44. Normalized conductance vs. bias; terrace and step ........ 162 
1. Calibration image of Bopg ..................................

2. Gold surface by nilver tip ......................... 166

3. Gold surface by platinum tip ...................... 168

4. Platinum surface by platinum tip .................... 170

5. Platinum surface without and with conductance texture ...... 172

6. BOPG Burface with conductance texture ................. 174

7. BOPG twist boundary defect ...................... 176 
LIST OF TABLES

\section{Table}

1. Gain and phase response of optimized experiment controi circuit $\ldots \ldots \ldots \ldots \ldots \ldots \ldots \ldots \ldots \ldots \ldots \ldots \ldots \ldots \ldots \ldots \ldots \ldots \ldots \ldots \ldots \ldots \ldots$

2. Model circuit values from admittance experiments on an inert

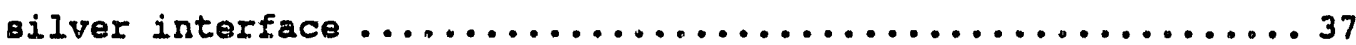

3. Comparison of experimental results and molecular dynamica results scaled to silver surface self-diffusion ..........48

4. Simulation results for several diffusion rogion lengths and

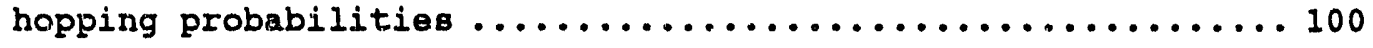


PREFACE

The focus of this work is the study of defects at surfaces and interfaces. Here, defects are defined as deviations from ideal ourface structure in the form of point and larger structural defects, steps and one-dimensional defects at steps, and physical adsorbates or chemical inhomogeneities which modify surface behavior. Defects are important because they are often more chemically reactive than other (more ideal) regions of Burfaces. An understanding of the nature and reactivity of defects is essential to the understanding of "realiatic" surfaces and interfaces that contain a mixture of ideal and defective regions. The approach here is to apply surface-sensitive detection techniques to study surfaces and interfaces with low numbers of defects in order to discover chemical, physical, or electronic information about those defects.

In general, our tool for probing surfaces and interfaces is the electron. Bere, electrons are not employed as surface sensitive scattering tools, as they are commonly used in surface science. Rather, high-precision electron counting is applied as a surface and interfacial probe. Present technology allows detection of electrons in "blocks" for sustained periods or as single electrons up to a limited, but considerable, charge. We apply the former technology and use special circumstances to attain high surface-sensitivity. This work presents two types of experiments in which electrons are counted by the currents that they generate. 
The first type of experiment uses the transfer of electrons to and from localized electronic states to measure the stages of a surface chemical reaction. Low-defect crystals of oilver are prepared by growing silver crystals down a capillary tube. These low-defect ourfaces are characterized by an interfacially sensitive technique, impedance spectroscopy. The surfaces of these crystals are then used as subetrates for the study of monolayer nucleation and growth. study of the reaction rate during discrete monolayer growth events net only reveals the kinetics of the growth reaction, but also information about defect site location and reactivity. These studies have also allowed what we believe to be the first direct determination of the diffusion coefficient of a metal atom at a solid/liquid interface.

The second type of experiment uses the elastic tunneling of electrons between delocalized electronic states to measure the structure and properties of conducting materials. These experiments fall into the categories of scanning tunneling microscopy and spectroscopy (STM/S), with which the reader may be familiar. We have constructed a sTM/s instrument that is optimized for acquiring simultaneous images of topography and conductance. Due the ambiguity in the interpretation of topographic data alone, we have found that the application of complementary techniques is required to obtain chemical, physical, and electronic information regarding defects at surfaces or interfaces. This thesis addresses these experiments in the following manner. Chapter one presents the background, experimental protocol, and results of the silver crystal growth experiments. chapter two focuses on computational issues that developed from the work in chapter one; 
namely, simulations of monolayer growth and arface diffusion under low aurface concentrations. Chapter throe describes our ucanning tunneling microscope and some experiments on "zealistic" arfaces in air. A sumary of all the work and some additional coments axe provided in the closing chapter, chapter four. 


\section{ACKNOWTEDGMENTS}

I would like to thank professor John D. Porter for his guidance and extensive coments on this manuscript. I would also like to thank professor Eugene Haller and Gabor somorjai for reviewing this thesio. special thanks go to the Porter research group and rina Rouse for their encouragement, and Maria E. Orive for hex apport, patience, and helpful comments on this manuscript. Funding for this work was provided by the Department of Energy through grant number DE-AC03-76sF00098, and by the Graduate Division in the form of a Regents Fellowship. Inage processing was conducted at the U.C. Herkeley College of chemistry Computer Graphica Facility, funded in part by NIB award 8sorRo5G5 IA. 
CHAPTER 1

\section{PREPARATION AND CEARACTERIZATION OF INOW DEFECT SILVER CRYSTAL SURFACES}

\section{Introduction:}

Experimental work in the field of ourface science has greatly added to the Eundamental understanding of many practical chumical syoteme1,2. Visualizing physical surfaces as more than mere planar boundaries between solids and other phases has been crucial to these advances. In the plane of the surface, the presence of deviations from ideal packing (defects) often leads to dramatically different chemical and physical behavioj in romparioon to the ideal ourface. It is very important, chen, to characterize the number densities, structuro and reactivity of aurface defects. Application of results from vacuua surface science to the understanding of real interfacial eystems can often be hindered by the comploxity of typical surfacen and interfaces. Details which must be considered when thinking about or describing such a aystem are numerous, making sample preparation and the choice of system of study critical. What is needed are experimente which can probe more complex systems yet still retain enough aimplicity to provide fundamental underatanding.

Defects at surfaces can dominate catalytic chemical bohavior in many circumstances. One class of examples are found in the area of nucleation and growth ${ }^{3-8}$. The rate of formation of a new phase from a supersaturated solution can be dramatically enhanced by macroscopic or microscopic defects. To model growth and deposition of thin films, it 
is important to understand the formation and reactivity of defect sites that promote growth.

Growth-promoting sites actually can take many physical forns such as point defects, atomic steps, kinks in oteps, and general stacking faults. Information regarding these sites can come from different types of experiments. At present, work in many areas has begun to address the roles of defect sites in broad terms ${ }^{5-8}$. In most systems, microscopic details of defect structure and reactivity are atill unknown. In modeling a real system, knowledge of the number density of these sites and their activity are critical clues to the time evolution of the gystem. Another key piece of informaticn to obtain is a physical map of the locations of these sites. Such a map could be a useful tool in the structural characterization of defect sites and investigation of structure-reactivity relationships. Our experimental work has been focused on the study of surfaces with almost perfect structure and the characterization of the remaining defects in these ideal systems. In the growth of homogeneous (like-upon-like) systems, meaningful information can be obtained about defect sites and their reactivity. These interfaces, either solid/gas or solid/liquid, have been the subject of research for a considerable period 4 . The most detailed experimental work studying microscopic growth issues has been done rather recently 9,10 , ccmpared to the historical interest in the field 11 . The process of growth can be summed up as the condensation of smald growth subunits from a supersaturated phase into the solid phase. supersaturation may be created in several ways. In the gas phase, a vapor or partial pressure of the atoms may be exposed to the substrate, 
resulting in bulk growth. In liquids, an electrochemical potential can provide a driving force for net deposition. The process of initiating bulk growth is the nucleation of a new solid phase on the substrate. Usually, certain types of defect sites on the surface activate growth via reducing the critical cluster size required for nucleating a new phase ${ }^{3}$. In this case, even modest supersaturations produce growth which proceeds in a regular, deterministic manner. occasionally, the defects on the surface can act as catalysts for growth through much larger critical clusters. Bere, the correct supersaturation conditions produce net growth by a series of many microscopic events. It is through the study of these microscopic events that defect reactivity and system growth dynamics can be determined.

Historically, microscopic growth models have centered around the dimensionality of the net growth ${ }^{12}$. One category of growth is characterized by the three dimensional growth of isolated islands on top of the substrate. This type of growth is known as volmer-weber growth. Another growth mechanism is the two dimensional expansion of atomic or thin flat layers. In this case, the new phase makes a homogeneous 2-D layer over the old phase. Note that this growth model does not stipulate that the growth in a particular layer must be completed before the growth in a new layer begins; it simply excludea the presence of isolated islands. This is the nature of Frank-van der Merwve type growth. Experimental evidence also exists to support a mix of these two categories. In this mix, the Stranski-Rrastanov mode, a few layers (2-D) are uniformly deposited over the substrate before the growth of the 3-D 
islands comences. One recently published example with good references can be found in the work by Taylor et al 13 .

selection between growth mechanism is often cletermined by the chemical system involved 12,13 . The surface energy differences between phases and the degree of supersaturation play key roles in determining the dominant growth mechanism. One cain generaily describe the competition of various growth models as a balance of lateral and vertical growth rates. In the growth of many homogeneous systems, surface defect type and amount of driving force for growth combine to determine the growth mechanism. It is possible to force systems into one mechanism or another via external. means which affect these variables. For heterogeneous systems, the same can apply. However, the surface energy differences between the two solid phases (substrate and new growth) control the early stages, producing in many cases, layer-bylayer growth followed by island formation.

one very important subset of the layer-by-layer growth model is the self-propagation of screw dislocations emerging at the surface of a crystal. A seminal work on this mechanism was published by Burton, Cabrera and Frank in $1951^{3}$. Screw dislocation defects are members of the class of defects which can promote growth via very small critical cluster size. The atomic step line extending from the emergence point of a screw dislocation provides a catalytically active site for growth. Growth may proceed at screw dislocations at significantly lower driving force or supersaturation than on planar defect free crystal surfaces. In general, auto-catalytic growth of steps proceeds at lower supersaturations then the nucleation of entire new phases. 
our system of study is the growth by electrochemical reduction of silver ions to form silver single crystal surfaces,

$$
\left.\mathrm{Ag}_{(\mathrm{ag})}++\mathrm{e}^{-}=\mathrm{Ag}_{(\mathrm{g})}{ }^{0} \quad \mathrm{E}_{0}=0.799 \mathrm{~V} \text { (VB.S.H.E.) }\right)^{14} \text { (1). }
$$

This is a simple and useful system for several reasons. The electrochemical reduction is quite reversible under typical conditions and is well behaved electrochemically. The very positive reduction potential of $\mathrm{Ag}^{+}$also tends to prevent co-deposition of many metal contaminants. Finally, large amounts of work on the silver reduction system has been conducted because it is perceived as a model system. General study of silver polycrystalline surfaces has been done by several other groups $9,15-20$, indicating interest in the system and its relevance. Work by the Bulgarian Academy of sciences towards low surface defect preparation of silver crystals has been conducted for several years $10,21-29$.

Research by Budevski and coworkers on silver single crystal growth has substantiated the claim that ultra-low defect single crystals may be prepared by capillary growth techniques 10,21,26. Silver single crystals (usually Miller index (100) planes) were placed against the mouth of 200 micron diameter capillary tubes. While electrochemically depositing silver on the surface, growth down the capillary was observed. In some cases, the authors observed that the growth rate in some cepillary tubes lowed dramatically. By studying the instantaneous rate of growth (the reduction current, the growth process was observed as a series of discrete events. Each growth event consiated of nucleation and layer 
growth processas. The layer heights were found to be monatomic by counting the number of electrons in each growth step. Our interests in this system center on reproducing these results and studying the microscopic growth of these thin metal films in detail.

Information which can be obtained from experimental studies of these silver surfaces falls into the following areas; adsorption, energetics and dynamics of adsorbed species, growth rates of thin films, and details of number density and activity of surface defects that affect growth. We have used a.c. impedance 30 techniques to measure equilibrium values for silver adatom populations and organization energetics of the interfacial double layer. Analysis of nucleation and growth events has revealed layer growth rates, shape of expanding growth islands and defect site locations. Finaliy, by studying sets of growth events, we have observed signatures of surface diffusion and quantified the results at two temperatures.

\section{Experimental:}

Three parts comprise this experimental section; electronics design, capillary cell design, and experimental techniques. Since the electronics used in these experiments were made in-house and reflect an original application of low noise analog circuit design and digital interfacing, a careful description will be provided. The experimental cells used here are of common design ${ }^{31}$; minor modifications are made to accomodate the capillary experiments. Experimental techniques discussed here cover both the preparation of low defect surfaces and their characterization in situ. Characterization techniques in this 
experimental context include a.c. impedance studies, equilibrium measurements of the nucleation process, and time dependent monitoring of the microscopic growth process.

In order to operate over widely varying time scales, considerable effort must be applied toward minimizing noise in the electronic circuitry. Fundamentally, these electrochemical experiments are based on the control and measurement of electrical potentials and responses to the potentials in the form of current. Noise in these signals will degrade the quality of the experiments and the results, so this is a very important issue in the design of the experimental apparatus. Two of the best defenses against laboratory electrical noise are careful shielding and good circuit design 32,33 . System noise can also be reduced by limiting the response window of interest, both in signal size and bandwidth of the signals. Our research in the design of these types of experiments has required the application of all of these tools and some linear averaging to reduce noise. Using the temporal noeds of a particular characterization method to determine the exact combinstion of the above techniques has been crucial in the battle between signal and noise. The system design required considerable flexibility, so the designs presented here not only represent a good solution to the problem at hand but also show ideas ready for ganeralization.

The basic problems which the experimental apparatus must solve are multiple and coupled. To control the electrochemical cell, a potentiostatic circuit with low noise is required. These experiments with silver nucleation and growth require a range of applied d.c. potentials of $\pm 100 \mathrm{mV}$ with a precision of $10 \mu \mathrm{V}$. Measuring the rate of 
growth is done by converting the reduction current into a voltage. For capillary tubes on the order of 100 microns in diameter, the total charge passed in the deposition of a monolayer metal film is about 10 nc. Over tine growth period of a Becond, this is an average of $10 \mathrm{nA}$. Our apparatus needed to handle currents up to $200 \mathrm{nA}$ with a precision of $0.05 \mathrm{nA}$. Previous experiments 10,21,26,27,29 of this type have used chart-recorder type technology to record long time series of growth events. In our work, we have interfaced a computer to the apparatus to automate cell control and data analysis. While this addition provideci, significant advance in the accessible data siot oize and enhanced data analysis capabilities, removal of the electrical noise caused by the computer required extra effort due to our small signal sizes.

Computers, fortunately, axe well suited to handling large volumes of data. Our expectations based on the previous work were that time series data should be acquired at rates of 1,000 to 10,000 times per second, for extended time periods. With the help of an ATsT 6300 plus (AT compacible) we were able to record up to 240,000 points at a time. The conversion from analog to digital signals was accomplished by a Labmaster (Scientific solutions) experiment interface board. signals to the analog-to-digital conversion system were locally buffered, preventing the computer inputs from providing an easy path for noise introduction. Our maximum rate of conversion, retrieval and storage of data points was at $10 \mathrm{kBz}$. This gave 24 seconds of run time for our best temporal resolution. Although adequate for recording a few growth events with very fine resolution, recording time series with many growth events required the use of a slower sampling rate. It is interesting to 
note that a personal. computer of similar price today would be able to attain higher sampling rates by at least a factor of 4 and total data point storage per experiment sixteen times larger. Our computer generated the experiment control signals via the Labmaster board. The digital-to-analog conversion was done with 12 bits of precision (i part in 4096), however the electrical noise of the computer contaminated the lowest 2-3 bits in wideband output situations. This was solved by increasing the rise time of the output signal to $10 \mathrm{~ms}$ via a passive low-pass filter. An additional step to reduce the impact of computer generated noise included the design of an internal adjustable voltage source, allowing the computer-based potential control to be disconnected during some experiments.

The small electrical circuits for the apparatus were constructed on industry standard double-sided copper-clad polycarbonate boards. All of the electronic circuits were placed on boards by signal type and function. Input potentials and local control signals were generated on the top-level board. This board had the most direct contact with the "outside" world. Immediately below, a board with two buffering circuits amplified small signals from the precision experiment control board to high levels. The experiment control board housed the potential control and current monitor circuits. In the initial experimental configuration, the experimental cell was also housed on this circuit board. The power supplies for these circuits were two 6 amp-hour motorcycle batteries. These chemical voltage sources provided atable and very low noise power for several days to the electronics. 
The electronics, experiments and power supplies were enclosed within a 1 cubic foot faraday cage. The Faraday cage was constructed out of copper-clad $1 / 4^{\prime \prime}$ mild steel plate and had holes drilled into the back with one inch spacing for input and output of electrical signals and thermal control fluid. This cage determined the design definition of "inside" and "outside" world signals. To prevent contamination of "inside" signals through direct and apacitive coupling, noise reduction techniques and careful shielding were applied on all signals from tho "outside" world heading in. The boards were mounted within the Faraday cage on a series of interconnected 15 pin connectors. The top-level card was connected to the "outside" world through BNC bulkhead mounts in the back of the Faraday cage. The buffer stage was connected to the top level card and the small gignal stage, and physically resided between those boards, just below the top-lavel card. In the following paragraphs I will present schematics and explanations of the building blocks for these experiments. The pin numbering is included here for completeness, but detailed descriptions of the individual integrated circuits are omitted since they are well discussed elsewhere ${ }^{33}$.

Illustration 1 is a schematic of the top level board. The task that this board was designed to accomplish is the control of potentials applied to the experiment. One circuit produces a square wave signal centered about zero volts. It uses a 555 timer to generate a $50 \mathrm{~Hz}, 360$ mV peak-to-peak signal when the relay enabling its output is closed. The timer is powered by the relay control signal, turning the oscillator on only when its signal is allowed to pass to the experiment. The output stage divider with $1.1 \mathrm{k} \Omega$ to ground is also the input for the 
computer based potential signal. The series resistance of that signal is $110 \mathrm{k} \Omega$ producing a 100 times division of the input signal. The combination d.c. signal from the computer and the square wave signal are propagated to the experiment control board together. The other component of this board is an adjustable negative voltage regulator. This produces a signal of 2 to $20 \mathrm{mV}$ at the output with correct sign to drive the reduction of silver ions onto the working crystal surface. A local potential source of this type provides very low noise and low drift operation by eliminating the need for external potential input. This signal is not combined with the other potential control signals on this board because an active component would be required to correctly add the small level d.c. signals. Part of the control small Bignal electronics conducts the summation.

Illustration 2 is a schematic of the two functional blocks on the buffer board. These are both simple operation amplifier circuits. The industry standard 741 amplifier is configured as an offset nulled noninverting $40 \mathrm{~dB}$ amplifier. This circuit buffered the output from the potential monitor on pin 6 . With nulling of this stage, measurement of the millivolt signals of the potentiostat could be accomplished with an accuracy of $5 \mu \mathrm{V}$. The adjustable feedback loop amplifier was used as both adjustable bandwidth and adjustable gain amplifiers during these experiments. As drawn, the relay position determines the effective bandwidth of the amplifier, keeping the amplifier gain constant. This amplifier acted as the buffer for the output of the current to voltage converter on the small signal stage. The basic purpose of this stage is to condition the outgoing signal toward the appropriate response 
bandwidth and noise level of the characterization technique. The relay position which selects the path containing the 2000 pF parallel capacitor is used for recording time-based, narrow bandwidth, low d.c. noise current data. The other position enabled faster response of this stage and served as the setting for the a.c. impedance experiment output source.

Illustrations 3 and 4 depict the circuits which required the most design effort. Illustration 3 is the three electrode potentiostat circuit used in most of the work reported here. The second complete board design represents the optimal circuit design for this type of experiment and was developed in steps during the experimental work. Both represent very simple, low noise solutions to experimenta which need to make precision current measurements in electrochenical systems. The three electrode circuit supports the typical counter, reference and working electrodes and works well with currents on the order of nA, controlling potentials with microvolt precision. The quality of the input signals and reference electrode type will usually determine the precision, rather than the circuit itself. This circuit maintains stability by using the cell as part of the feedback for the potential control amplifier. This suming amplifier takes inputs of d.c. and a.c. voltage and generates an output within the experimental cell that produces a match to these inputs at the location of the reference electrode. The control amplifier in this design is able to source $10 \mathrm{~mA}$ and $10 \mathrm{v}$ to accomplish this task. The amplifier connected to the reference electrode acts as a buffer to the signal returned from the experimental cell. The output of this buffer goes to pin 6 where it is 
amplified to "outside" world levels. The amplifier attached to the working electrode convert the current running through the cell into a voltage. The transimpedance gain of this block is $2 \times 10^{7} \mathrm{~V} / \mathrm{A}$ while the mall "ignal response bandwidth in around $2 \mathrm{kgz}$. Bowever, this responde widts strongly depends on the total reaigtance in the experimental cell. When the cell configuration wa modified to reduce the capillary length and its resistance, this circuit design proved to be too slow in response to allow frequency domin expariments. Despite its slow performance, the analog devicen AD549 low input current amplifier delivered outetanding time domain response and exceeded our needs for a nA current measurement device by a fair margin.

The original three electrode potentiostat circuit evolved into the two electrode apparatus in illustration 4. Our symetric and chemically simple aystem made the use of a reference electrode redundant when little or no current was being passed through the cell. By using local foedback to the suming control amplifier, the noise above $1 \mathrm{kHz}$ to the cell was dropped by about 5 times. The amplifier used in the current measurement otep in a very fast, low input current uncompensated op-amp. This renulted in oignificantly widening the bandwidth of the current meanurement. The high impedance lead from the experiment had to be Lurther shielded to prevent thi bandwidth widening from significantly degrading the final output quality. The additional shielding was accomplished by bootstrapping a guard around the Bignal laad to the potential of the lead. This is a common trick used to decrease the apparant impedance of a line and reduce its antenna-ilike behavior. This converted the original coaxial wire, (inner bignal with an outer ground) 


\begin{tabular}{|c|c|c|c|c|c|c|}
\hline$R_{\text {Cel1 }}$ & \multicolumn{2}{|c|}{$0 \Omega$} & \multicolumn{2}{c|}{$50 \mathrm{k} \Omega$} & \multicolumn{2}{c|}{$100 \mathrm{k} \Omega$} \\
\hline $\mathrm{f} / \mathrm{Bz}$ & $\mathrm{Gain} / \mathrm{dB}$ & phase & Gain/dB & phase & Gain/dB & phase \\
\hline 10 & 0.0 & 1.2 & 0.0 & 1.0 & 0.0 & 0.8 \\
\hline 100 & -0.027 & -2.0 & -0.033 & -2.3 & -0.0059 & -2.3 \\
\hline $1 \mathrm{k}$ & +0.011 & -2.6 & -0.049 & -4.5 & -0.071 & -4.6 \\
\hline $5 \mathrm{k}$ & +0.76 & -9.6 & -0.58 & -11.6 & -0.71 & -11.7 \\
\hline $10 \mathrm{k}$ & +0.53 & -31 & -1.3 & -16.6 & -1.6 & -15.5 \\
\hline $20 \mathrm{k}$ & -3.3 & -50 & -2.4 & -22.6 & -2.5 & -19 \\
\hline $50 \mathrm{k}$ & -8.9 & -53 & -4.6 & -31.9 & -4.1 & -27.5 \\
\hline $100 \mathrm{k}$ & -12 & -58 & -6.9 & -44.7 & -6.0 & -40.8 \\
\hline
\end{tabular}

Table 1. Gain and phase response of optimized experiment control circuit. The gain is reported in dB shift from low frequency values. Phase is reported in absolute shift in degrees from the input signal.

to a triaxial wire of signal, guard, and then ground. The response bandwidth of this design was orders of magnitude better with only a factor of 3 increase in d.c. noise for very low cell resistances. A sample of the response curves vs. Frequency for a model experiment characterized by a simple resistor in parallel with the input $10 \mathrm{k} \Omega$ resistance is shown in Table 1. The $10 \mathrm{k} \Omega$ resistor is used to set a bound on the instrument response which can be calibrated. The flat, 6 to $12 \mathrm{~dB} l 08 \mathrm{~s}$ over $100 \mathrm{kBz}$, response is nearly the best possible for circuits which set out to measure currents on the order of nA and smaller. However, the application of this design is limited to aystems where two electrodes are sufficient and the presence of an uncompensated resistance of $10 \mathrm{k} \Omega$ is acceptable. 
To close the description of the electronic apparatur, an outline of the computer operations and software follows. As mentioned earlier, the computer controlled the experiments and data acquisition. computer control cane in the form of both analog signals to the cell and drive signals for the relays on the circuit boards. The bulk of the data acquibition here was retrieval of digital data from an analog to digital converter. This data was saved in the computer's memory. Once the memory capacity was filled the data were written to files on a hard disk. The hardware link between the computer and experiment was established with the Labmaster board. This board was mounted within the computer and provided the digital Bignals, analog outputs and inputo noeded. The digital oignalo were amplifiad in capacity by transistors to drive the relays. One of the board's analog outputs was filtered and sent to control cell potential. The analog inputs converted both current and applied potential signals into digital values with 12-bit precision. The software to operate the hardware link was written and complied in the Microsoft QuickBasic 4.0 environment. source code for the experiment operation, data acquisition and data viewing is included in Appendix A. A program designed to control and monitor the early stages of growth of the crystal down the capiliary was developed, GROWSAMP.BAS. A single integrated program WRRSPCE.BAS ran all experiments and controlled steady atate cell conditions via a menu interface. The size of the control program was kept emall to allow for maximum storage within the computer memory space. The control program ran potential step and constant potential growth experiments directly. It sent control signals and ran experiments with a Princeton Applied 
Research model 5208 lock-in amplifier through a GPIB interface card (National Instruments PC-IIA). Another program, FILEPLOT.BAS was employed to read and scan through saved time-series data. This program generated output on a Bewlett Packard $7475 \mathrm{~A}$ plotter via the GPIB interface.

The experimental cell used in these experiments was a threaded teflon cap with a glass bottom, providing a total volume of $10 \mathrm{~mL}$ (see illustration 5). The teflon cap was machined to accomodate three $1 / 16^{\text {" }}$ diameter tube swagelock fittings. Two holes were on the side of the cap; these served as inlet ports for the counter and reference electrodes. The last hole was in the center of the cap, allowing a struight path through the cap for the working electrode. The base of the cap had a $6 \mathrm{~mm}$ diameter hole which provided a mounting aite for the capillary tubes. The glags and teflon parts were easy to clean and provided inert containers for the concentrated silver nitrate solutions used in the experiments. During the course of experiments, reference electrode configuration and capillary type were varied.

The counter and reference electrodes were constructed from $0.25 \mathrm{~mm}$ diameter silver wire (Alfa 99.98). The counter electrode was a bare wire inzerted through the teflon cap aftex cell filling. The reference electrode evolved during the course of the experiments. Originally, all but the tips of the silver reference electrodes were sealed in glass. A bend was placed in the glass to position the end of the electrode at the capillary mouth. Most of the glass seals leaked, however, and the reference electrode in later experiments was another bare wire bent towards the capillary mouth. In fact, potential control through the 
reference was at times unreliable; this issue is analyzed in the discussion.

The working electrodes were designed to provide an electrochemically inert surface that would act as a substrate for the growth of silver seed crystals. One good material for this is platinum. platinum wires of small diameter (less than $.20 \mathrm{~mm}$ ) are easy to seal in ordinary or hard glass. Oux working electrodes were constructed of short lengths of .125 m diameter pt wire sealed in glass tubes. One end of the wire was completely embedded in glass while the other end was attached to a larger diameter silver wire. The silver wire extended out the unsealed end of the glass tube, providing contact to the Pt electrode. The embedded end of the Pt wire was exposed by a series of polishing steps. The coarse step was done with 400 to 600 grit sandpaper until a regular disk of platinum was exposed and surrounded by glass. The remaining steps were polishing this surface with a series of decreasing size alumina particles. The particle used ranged in size from $1.0 \mu \mathrm{m}$ down to $0.3 \mu \mathrm{m}$ and they were supported on ordinary filtex paper. Methanol was used to prevent the particles from sticking together. The polishing process was checked for completeness between steps with a $100 x$ optical microscope. The final surfaces appeared mostly flat, with some detectable scratches. Overall, the surfaces had a fair mirror finish after 20 minutes of polishing. A well polished and cleaned Pt electrode resisted nucleation of silver crystals to overpotentials of $30 \mathrm{mV}$ with respect to a silver polycrystalline wire. These surfaces were sufficiently inert to allow the formation of only a single crystallite, and rot multiple crystals during seed growth. 
Two capillary configurations were used. Initially, capillary tubing from wilmad Glass was employed. These tubes had a total length of $0.5^{\pi}$, an outer diameter of $6 \mathrm{~mm}$, and a bore diameter of $76.2 \mu \mathrm{m}$. When filled with electrolyte, the capiliary resistance to current flow of these tubes was quite large because of their length. To obtain capillary tubes with lower resistance, short capillary tubes were made by spinring the end of $6 \mathrm{~mm}$ Pyrex glass tubing in a torch flame. In the flame the end of the tube melted and collapsed, producing capillaries of length 1-2 $\mathrm{mm}$ with bores comparable to the commercial capillary tubes. The capiliary tube had an hourglass shape after formation (see illustration 6). Black wax was pulled into the capillary to prevent damage to the tube during abrading and polishing. The capillary was abraded until the region with the narrow bore was exposed at the end. The end of the capillary was then polished to a smooth finish. Finally, the wax was removed from the capillary by combustion in a flame. The capillary tubes of both types were cleaned first in methanol saturated with potassium hydroxide ( $\mathrm{KOH} / \mathrm{MeOH}$ ) and then in agua regia. After this the capillaries were rinsed with ultrapure water and exposed to dichlorodimethylsilane $\left(\mathrm{Cl}_{2} \mathrm{Me}_{2} \mathrm{Si}\right)$ by placing them in a $150 \mathrm{mI}$ beaker with one drop of the solution and covering with parafilm. After 6 to 24 hours the capillaries were hydrophobic, as verified by failure of capillary action with water.

Temperature control of the experiments was obtained by exposing the cell to a solution circulated in a thermostat bath (Neslab model RTE-5DD). The solution used in the bath was a $1: 1$ Prestone anti-freeze to water mixture. This bath solution was circulated around the cell two 
ways in these experiments. Illustration 7 shows the cooling/heating schemes. The plexiglass jacket probably provided better temperature control since there was direct contact between the bath solution and the experimental cell. The copper coil worked by proximity thermal control alone and required additional insulation (cotton gauze was wrapped around the coil). However, use of the copper coil and the short capillaries allowed optical observation of the growing silver crystals throughout a given experiment. The objective of a $100 x$ microscope was placed in one open end of the copper coil. This microscope could focus over the entire length of a propexly positioned and polished short capillary. This configuration significantly enhanced observation and quality control while meeting all of the other experimental design parameters.

Initial seeds for the experiments at the Bulgarian Academy were large silver single crystals. To place the erystals close to the mouths of the capillary tubes, however, these high quality crystals were sanded to fit. This rough treatment can damage the crystal significantly due to ailver's low energy of activation for slip plane $\mathrm{f}$ ropagation 34 . We developed techniques to grow small low defect seed crystals ex and in situ on platinum and other small electrodes. For silver, these high quality single crystals can be grown to dimensions on the order of a few hundred micrometers across. On Pt disk electrodes sealed in glass, the seeds were grown from an aqueous solution of 3-5 $\mathrm{AgNO}_{3}$ containing 0.1 mM $\mathrm{BClO}_{4}$. A single microcrystalline seed was formed on the Pt electrode by the application of a $-30 \mathrm{mV}$ potential (with respect to a polycrystalline silver wire) to the clean electrode until current begins 
to flow. Typically, the background current is less than one nA, and once the silver crystal begins to grow, the current ribes above $50 \mathrm{nA}$. Higher initial potentials tended to produce multiple separate cryotals. Once the current started flowing, good crystal geometries were formed for growth potentials between -15 and $-20 \mathrm{mv}$. Total charge passed per seed crystal was between 1.0 and $20 \mathrm{mc}$. The seed crystal morphology and quality were checked with a $100 x$ optical microscope. Optically, the seed crystals appeared to be bounded by flat facets with sharp boundaries between facets. Six sided facets usually dominated these microcrystals although some rectangular to square facets were present. These crystals were also grown on a small diameter carbon fiber but the adhesion to the Pt surface was much better. For seeds grown on platinum, it was possible to lift the whole glass electrode by placing the tip of a razor blade against the seed crystal. This turned out to be a convenient check of the adhesion of the seeds, although it was usually used to check preparation techniques rather than test individual seeds.

The cell equipment was cleaned in a saturated potassium hydroxide in methanol solution followed by aqua regia. Parts were rinsed in ultra-pure water (Millipore Milli-Q system) and the teflon cap was kept in $\mathrm{B}_{2} \mathrm{O}$ overnight to leach out chloride ions adsorbed while in the aqua regia. The experimental cell was filled with a solution of concentrated silver nitrate by a syringe. All of the experiments used $5.0 \mathrm{M} \mathrm{AgNO}_{3}$ prepared from pure crystals (Fisher A.C.S.) and ultrapure water. Near the end of the experimental work, varying amounts of porchloric acid were added to study low $\mathrm{pH}$ versus nominal $\mathrm{pH}$. In addition to varying 
$\mathrm{pH}$, the oxygen content of some of the solutions was reduced by bubbling with $\mathrm{N}_{2}$ for 10 to 20 minuter. Before filling, the cell. was assembled by screwing on the teflon cap with capillary tube and reference electrode installed. A teflon syringe tube was passed through the counter electrode entry hole to fill the cell. To get the solution to enter the hydrophobic capillaries, a small amount of vacuum was applied to the top hole in the teflon cap, sucking the solution up the capillary. After the cell was filled, the counter electrode and the working electrode wi:n seed crystal were installed. Care had to be exercised to prevent bumping the working electrode and shearing the seed off the end of the electrode. For the long capillary tubes, the seed and capillary could not be seen after assembly because the end of the capillary was within the teflon cap. With the shorter tubes, guality control was much better.

Once the seed was placed at or near the capillary mouth, the cell was mounted within the Faraday cage and connected to the control electronics. A d.c. potential was applied to produce net growth while a larger amplitude square waveform was applied at a frequency of $50 \mathrm{kz}$. some experiments assessed the effects of potential applied and the frequency and magnitude of the oscillating potential. The effects of cell temperature on growth morphology in the capillary were studied in the range of 0 to $60^{\circ} \mathrm{C}$. Typical conditions used in the initial growth segment were applied potential of $-25 \mathrm{mv}$, oscillator amplitude $200 \mathrm{mV}$ peak-to-peak and thermostat bath temperature $40^{\circ} \mathrm{C}$. The crystal was grown down the capillary until signs of reduced surface defect density appeared in the current response. In the long capillary tubes, thi.s 
occurred over few millimeters from the capillary mouth. In the shorter capiliary tubes, the crystal often grew through the full length of capillary and out into the main solution volume. The ideal capillary length is probably on the order of 2-5 mm, between the lengths that we had at our disposal.

The current response to the applied square wave was one guide used to determine the reactivity and defect density on the growing cryatal. The response of a simple resistive cell to the input square wave would be a muare response plus a current from double layer charging at the interface. For a surface with a large number of active sites or a few exceptionally active sites, this type of response was observed. When the number or activity of the growth promoting sites decreased, the response to the applied square wave changed. Illustration 8 shows a typical comparison of these two situations as viewed by an oscilloscope. The change in response is due to a change in the growth mechanism. When nucleation of new layers or nucleation of pits at the interface become an important part of the net reaction, the temporal response takes on a slower, delayed form.

Another guide that we used to study defect density and reactivity was descriptive statistics regarding the instantaneous growth rate. During the growth of the crystal within the capillary, the computer changed the applied potential from $-25 \mathrm{mV}$ to $-6 \mathrm{mV}$ and turned the oscillator off at regular intervals. After a relaxation period of four minutes, the computer acquired a large number (ca. 5000) of readings of the current over the course of a minute. The program then printed out the applied potential, the mean current, the standard deviation and some 
other descriptive statistics. After printing out the data, the growth experiment was resuned at the previous conditions. The computer automated long experiments through this growth data sampling program. Usually, the data sets were acquired every 30 minutes with total run time of the experiment in the long capillaries of a few days to one week. By looking at the mean current, a quick estimate of the reactivity of the surface could be obtained. Bowever, this one value did not describe the time-based behavior. For example, one could not tell the difference between alow growth caused by a broken contact vs. slow growth due to an inert wurface. During periods of slow growth on low defect surfaces, a major component of the growth current was due to fluctuations caused by microscopic nucleation and growth events. The normalized deviation and skewness of the data were used to measure the magnitude of these fluctuations and their deviations about the mean. Armed with these tools and techniques, we went in search of the "fake-atom smooth"21 surface.

First observations of non-typical interfaces appeared in the form of non-reactive or inert surfaces. This behavior was first recorded and studied by slow potential sweep experiments. A computer program was written to look for signs of the onset of growth activity of these interfaces. The program started at a small reductive potential, -1 to $-3 \mathrm{mV}$, and incremented the potential in small steps with a few seconds between each step. This produced a steady state potential sweep experiment. From these experiments it was possible to measure the overpotential required to trigger growth. These data also allowed an estimate of the rate of supercritical cluster formation at the onset 
potential. The inert interfaces were oubjected to large amplitude potential pulses of short temporal duration to "jump-start" the growth process.

Inert interfaces were also characterizad by impedance spectroscopy techniques. An oscillating perturbation of $33 \mu \mathrm{V}(\mathrm{rms})$ was applied to the counter electrode in addition to the d.c. overpotential. The current response to this signal was detected by a Princeton Applied Research two-channel lock-in amplifier (PAR model 5208). The response signal was recorded as the $X$ and $Y$ admittance or, equivalently, as a magnitude $|Y|$ at a phase angle $\theta$. To obtain a response spectrum, the frequency of the a.c. perturbation was varied from $3.6 \mathrm{Bz}$ to $100 \mathrm{kHz}$. Usually, three to four points per decade of frequency were acquired by hand. A computer routine was later added to the growth control program which measured five to ten points per decade of frequency. Either way, the total time required to measure the impedance spectrum was about 15 minutes. During temperature dependence studies of the impedance spectrum, about 15 minutes were allowed for equilibration at the new temperature. Background instrument response to simulated experiments were measured with the substituted calibxation circuit and electrical leads positioned as during the experiments.

Observation of transient growth events and fluctuations was not uncommon in our experiments. However, surfaces which grew by distinctly separate events along a background of zero reactivity were very rare. These surfaces were recorded in action by the growth control program described earlier. In a few cases, several minutes of growth events occurring on a very low current background were recorded. Analysis of 
these series of growth events showed that the active ourface area could be best described as in illustration 9 . For studies of these minisurfaces, we applied mall constant potentials with the cell under thermostatic control. Attempts to change temperature while these metastable systems were active were unsuccessful because their period of activity was very short. In systems showing fluctuations in the growth process, some potential dependance otudies were attempted. Again, the difficulties of maintaining the activity of these metastable systems prevented significant insight.

Besulte and Discusgien:

In the study of growth in this system, we varied the growth parameters and tried to verify effects that these changes caused. visual and electronic clues were used to optimize growth conditions in order to produce large, flat single crystal surfaces within the capillaries. These surfaces were four and six sided, corresponding to low Miller index surfaces $(100)$ and (111) in the face-centered-cubic (fcc) system. Usually, side facets of the other orientation were observed bounding a central plane and forming the contact with the capillary wall. The regults in the following section degcribe our observations and conclusions on these close-packed surfaces. The crystallographic orientation of the active and inactive surfaces was not directly obtained within the capillary; rather, visual clues based on surface morphology were used.

The flat crystal planes within the capillary demonstrated three classes of growth. Often, the surfaces behaved like a typical 
polycrystalline surface, showing reversible, regular growth and dissolution around the equilibrium potential of silver. A small number behaved as inert surfaces, showing no reactivity over a range of potentials around the silver equilibrium potential. A few surfaces fell between these limits, demonstrating isolated, monolayer and multilayer growth events at small overpotentials. These three classes of growth appear to be tied into the presence or absence of growth enabling sites on the surface. The inert surfaces were characterized by the use of steady-state potential ramps, growth inducing potential pulses, and impedance spectroscopy. The slightly active surfaces were studied through observation of time-dependent growth rates at constant overpotentials.

The experimental conditions used by previous workers to prepare low defect silver crystal surfaces have almost an empirical "recipe" quality10,21-29. Consequently, we investigated a small range of growth parameters around the values traditionally used in order to obtain insight on the physical processes involved. To obtain the filling of any region, the relative horizontal (transverse to capillary) and vertical (along the capillary) growth rates are important. The horizontal growth rate should be much larger than the vertical rate to ensure that capillaries are filled as the crystal grows down the capillary. One of our interests then, was to explore conditions which enhanced the horizontal growth rate with respect to the vertical growth rate. Parameters which were studied in growth experiments were the cell temperature, applied potential, oscillator signal, and solution composition. These are ordered based on total observed effect on growth 
behavior. Temperature had the greatest impact on the overall quality of the crystals and capillary filling in these experiments.

Temperatures were varied over the range of 0 to $60^{\circ} \mathrm{C}$. The lower limit for cell solution freezing was around $-6^{\circ} \mathrm{C}$. Since the cell was surrounded by aif at roam temperature, temperatures above $60^{\circ} \mathrm{c}$ were not explored because of the large temperature difference between the cell and the ambient. Additionally, the significant changes to observed growth reached limiting values above $50^{\circ} \mathrm{C}$. There were very striking differences in the horizontal growth rate between room temperature and about $40^{\circ} \mathrm{C}$. These differences were observed in the rate of growth of sratli isolated ourface clusters and in the overall growth response. The current transients describing the microscopic growth processes on the surface appeared sluggish below $20^{\circ} \mathrm{C}$ and quite fast above $40^{\circ} \mathrm{C}$. Actual kinotic parameter for monolayer expansion rates were difficult to determine because of geometric effecta. Qualitatively, for the same applied potential, the horizontal growth rates scaled in a linear manner with temperature in degrees celsius. A change of a factor of 2 is significant when observed in the time domain, our method of observation for bytem demonstrating growth activity. As long as the temperature was held above $40^{\circ} \mathrm{C}$, capillary filling was acceptable. Not all crystals filled the capillary at this temperature, but this temperature marks a point of diminishing returns for further temperature increases. At the high growth rates used in the beginning of the experiments, this temperature kept the growing crystal from becoming a thin strand in the capillary bore. At lower growth rates, it was posible to maintain a filled capillary with lower temperatures. 
Applied potential also had a strong effect on growth rates, observed surface morphology and capillary filling. The magnitude of the growth overpotential determines the relative roles of horizontal step expansion in layers and the nucleation of new layers. The competition here is complex but a gualitative comparison is possible. At very low applied potentials, growth by the propagation of a single screw dislocation produces whiskers of 10-50 $\mu \mathrm{m}$ diameter. These potentials are in the range of -1 to $-2 \mathrm{mV}$ with respect to equilibrium. At low overpotentials, ( -3 to $-10 \mathrm{mV}$ w.r.t. equilibrium) the nucleation af two dimensional surface layers can successfully compete with screw dislocation propagation. These potentials were sufficient to obtain moderate capillary filling. At larger overpotentiala, growth is rapid within the capillary, and the extent of filling is reduced. This could be due to the tendency to shift from two dimensional nucleation of layer to the three dimensional nucleation of clusters on the ourface. During the early stages of growth from the seed erystal, growth was conducted at potentials of -15 to -30 w.r.t equilibrium. Final capillary filling and two dinensional growth studies were carried out with overpotentials of -3 to $-10 \mathrm{mv}$.

Physically, the application of and control of these potentials was not triviad. This was caused by two important factors, capillary resiatance and definition of an "equilibrium" potential. capillary resistance was merely caused by resistance to jonic charge transport along the very narrow capillary bore. A capillary of diameter $76.2 \mu \mathrm{m}$, length $0.5^{\prime \prime}$ filled with 5.0 AgNO 3 has a capillary resistance of about $130 \mathrm{k} 2$. Any net current through the cell produces a change in the 
actual applied potential at the silver/aqueous solution interface by Ohm's law. For a $20 \mathrm{nA}$ current, this potential drop is about $2 \mathrm{mV}$. The effect on growth kinetica of this voltage drop in maximized at the current maxima of the growth events. The events which we analyze here were observed at lower peak currents and smaller capillary resistance, unlike previous work $10,21-29$. The other problem observed was that the crystals were about 5 to $7 \mathrm{mV}$ more stable than the silver wire reference electrodes. While the crystal was growing readily within the capillary, the equilibrium potential could be measured by decreasing the overpotential until current ceased to flow. The instability in equilibrium potential could arise from several possibilities. Most likely, changes in the close-packed surface facets' contributions to the exchange current is the cause. On a low defect surface where anodic current (stripping) must be catalyzed by nucleation of pits, one would expect greater stability than on defect-rich polycrystalline surface. In light of these equilibrium uncertainties, it was only possible to compare small potential changes within an experiment. Overall, comparison of applied potentials between sets of experiments was not possible.

An alternating potential in the form of a square wave was usualiy applied during the process of growing the crystal down the capillary. Previous workers had used a large ratio between the net growth potential and the peak oscillator potential22,23. Typical signal ratios were in the range of $8: 1$ for pulse height to applied growth potential. We studied ratios in the range of $2: 1$ to $10: 1$ for these signals. Pulse rates of $1 \mathrm{gz}$ and $50 \mathrm{gz}$ were studied. The overall effect of the 
oscillator was subtle; however, it did prove to be a useful diagnostic tool at $50 \mathrm{kz}$ as discussed earlier. At the lower frequency, it was much more difficult to isolate the behavior of low activity surfaces since most of their unique kinetics are finished in 10-20 ms. We found that the ratio of the applied square wave magnitude to the growth potential was not very important. However, the absolute magnitude of the pulses had to be above $100 \mathrm{miV}$ peak to peak to suppress serew dislocation propagation and encourage capillary filling. By allowing potentials large enough to nucleate a new layer or a pit, the pulses can decrease the significance of screw dislocation propagation at low overpotential. The amplitude of the pulses required to accomplish this will depend of the temporal duration of the pulse. For the $20 \mathrm{~ms}$ wide pulaes here, 100 mV peak-to-peak was oufficient.

Cell solution composition was briefly investigated. While retaining a constant high concentration of $\mathrm{AgNO}_{3}$, both the oxygen content and the olution $\mathrm{pg}$ were varied. Solutions were deoxygenated before experiments by bubbling dry nitrogen for up to 10 minutes. After assembly, the cell was sealed to prevent contamination by oxygen. No diffexences were observed in crystal morphology or extent of capillary filling between experiments where the solution was dooxygenated and experiments where the solution was untreated. The reduction potential of oxygen in solution was negative enough so that oxygen reduction was not observed, preventing background currents in these experiments. The effects of low to moderate $\mathrm{pH}$ were studied in later experiments. High PE conditions were avoided because of the tendency of some ailver crystalline faces e.g. (110) to form silver oxide in alkaline 
solutions 14,18. The $p$ B was lowered by spiking with a solution of high purity perchloric acid. Again, no atriking differences were observed over a range of solution $p$ from 3 to approximately 7 . During the course of experiments, the low $\mathrm{pH}$ solutions were used to provide a controlled and low hydroxide ion concentration.

The observation of nucleation and growth on inert surfaces generally consisted of two techniques. Short timescale experiments provided information on the limiting kinetic processes and long timescale experiments yielded equilibrium measurements of growth onset. In these long timescale experiments, growth effectively "turned-on" over a window of a few millivolta. In our work, the activation of an inert surface could be classified as a catastrophic event occurring at a large overpotential. This differs from previous work where fluctuations were seen in growth at lower applied overpotential 10,26,27,29. Two possible models which can explain our observation of catastrophic events follow. One explanation may be that the surface is contaminated, resulting in a deactivation of surface sites. Another explanation consistent with previous work is that growth within the capillary has eliminated a clas? of surface sites which are active at low overpotential. In either case, it is completely reasonable to analyze our results at these two timescales to obtain kinetic and energetic data on these inert. interfaces.

Illustration 10 shows results from a pair of runs of the long timescale experiment. The top curve is a plot of the physical limit, $V=i R$, where $R$ is the capillary resistance $(109.7 \mathrm{k} \Omega)$. At large potentials, both of the experimental curves approach this limit. one of 
the experimental curves is the growth of a polycrystalline wire limited by a resistor equal to the capillary resistance. The other curve is the growth of the inert surface. On this logarithmic current scale, the observed growth is "zero" until some leakage charge begins to pass. These charges are fractions of a monolayer and may reflect the adBorption and charge transfer of precursors to growth. Possible precursors would be silver adatoms and their surface concentration should increase with potential. Once the potential exceeds a critical value, the growth current makes a sharp transition to the physical limit.

The growth of the polycrystalline wire, on the other hand, does not show onset behavior. These experiments were conducted with the same Bolution, in a comparison of the working electrode (cryatal in capillary) and the reference electrode. These two different results would inply that surface contamination from solution species is not playing a significant role in the observation of inert behavior. For kinetic analysis, we are able to acquire single data point, the onset potential and an estination of the nucleation rate at this potential. The large amplitude pulse experiments to study limiting nucleation rates were simple two-step experiments. From an initial potential where no current was flowing, a 50 to $300 \mathrm{mv}$ pulse was applied. After a short period, the potential was returned to the initial potential and the current was recorded to look for growth triggered by the pulse. The slow pulses between 1 and $50 \mathrm{~ms}$ long were generated by the tontrolling computer and shorter pulses were made with the help of a waveform generator. Large potentials are applied to form surface precursors 
which combine to form clusters on the surface. When a cluster is large enough to continue its growth even at the lower potential, a new phase has successfully nucleated and the cluster will grow and produce a current. By stepping to arbitrarily large potentials, we attempt to measure the absolute rate of cluster formation, independent of surface precursor issues. For the range of potentials that we usad, the typical pulse width that produced a growing cluster on more than 508 of the pulses was 1-5 ms. Extending this to an arbitrarily large potential, the limiting rate is on the order of $10^{10}$ per second $\mathrm{cm}^{2}$.

The two results outlined above can be applied to nucleation theory to determine energetic information relevant to the solid/liquid interface. A simple derivation from an equilibrium view of the surface gives the following expression for the rate of nucleation of a new twodimensional phase of height $h^{8}$. (See appendix $B$ for an outline of the derivation)

$$
A=A_{0} \cdot \exp \frac{\pi \frac{v}{h} \cdot \varepsilon^{2}}{\left[\frac{y}{h} \cdot \sigma_{13}-z e \eta\right] k T}
$$

where $A_{0}$ is the limiting rate of nucleation in nuclei per second $\mathrm{cm}^{2}$, $E$ is the edge energy of the new phase in $\mathrm{J} / \mathrm{cm}, \eta$ is the overpotential, and $\sigma_{13}$ (in $\mathrm{J} / \mathrm{cm}^{2}$ ) is the Burface tension between the substrate and the new phase. For an experiment that probes the nucleation of a like phase on a clean, defect free Burface, this surface tension term will be identical to zero. The surface energy term can be stabilizing or destabilizing for a non-j.deal surface and unsymetric systems. Taking 
our results to be representative of the former, we can calculate the edge energy of a two-dimensional cluster of silver. We obtain $\varepsilon=12.7$ $\pm 0.2) \times 10^{-13} \mathrm{~J} / \mathrm{cm}$. The error bar is small because the nucleation rate is explosive with potential, fixing $\varepsilon$ to a high degree even with oignificant experimental uncertainty. This result is similar to resulto obtained by Budevski et. al. in silver screw dislocation propagation experiments $\varepsilon \sim 2.1 \times 10^{-13} \mathrm{~J} / \mathrm{cm}^{21}$ and $\varepsilon-2.7 \times 10^{-13} \mathrm{~J} / \mathrm{cm} 10$. Illustration 11 shows the onset data point and its uncertainty and the model curve for our $\varepsilon$ and net $\sigma_{13}$ equal to zero. The vertical axis in this graph is the logarithm of the nucleation rate; our onset data is rate of about one event on the surface in 10 seconds. At lower applied potentials, the model curve based on this data falls sharply, producing very long time periods, > $10^{10}$ seconds, between nucleation events on these inert surfaces at applied potentials less than $8 \mathrm{mV}$. To place our results in the context of previous work, we propose that nucleation rates are enhanced by the atabilization of aurface clusters at defect sites. Previously published results on silver are schematically represented by the ellipse in the upper left of the graph in illugtration 11. A line of the model equation (2) with our determined $\varepsilon$ and $\sigma_{13}=-4 \times 10^{-2} \mathrm{~N} / \mathrm{m}$ passes through this region. This stabilization energy is a 58 change in silver's bulk surface energy, yet it produces a rate enhancement of many orders of magnitude. These results provide new information regarding the microscopic balance which affects nucleation and growth. The interpretation of growth through an energetically distributed active site model is also being purbued by 
Deutscher and Fletcher in the modeling of growth on assemblies of electrodes 7 .

The inert silver interface also provides an important starting point for other investigations of interfacial properties. The key to this is the low chemical reactivity of the surface. Impedance spectroscopy is a useful tool for examining interfaces. An impedance spectrum of one of these inert silver surfaces is shown in illustration 12. We have acquired these data over several temperatures and analyred the experimental response in terms of physically reasonatle processes at the solid/liquid interface. The low chemical reactivity of these silver crystals greatly assisted in parameter fitting and analysis. As measured, the spectra contained both experimental signal and instrumental distortion. The instrumental distortion was corrected via calibration circuits with known response. Calibration circuits were designed to approximate the response of the experiment based on leas distorted parts of the impedance spectrum. The differences between the observed response and the calculated response of the calibration circuit were used to correct the instrumental distortion. If required, this corrected data set was used to create an additional model circuit of greater accuracy or sophistication. The validity of this technique was checked through the measurement of a complex, yet known, test circuit. Later versions of the electronics were designed to reduce instrumental distortion. Studies with these improved electronics were done to verify that our experimental model circuit was appropriate. As this was found to be the case, experiments over a wide range of temperatures were not repeated. 
The interfacial admittance is shown in illustration 12 as logarithmic in magnitude and linear in phase anale (secondary vertical axis) vergus the logarithm of the perturbation freguency. When using a range of frequencies this form of plot is significantly more informative than the more traditional plot of imaginary impedance vs. real impedance. We are able to model the response of the inert interface over the temperature range of 0 to $45^{\circ} \mathrm{C}$ with the following equivalent circuit:

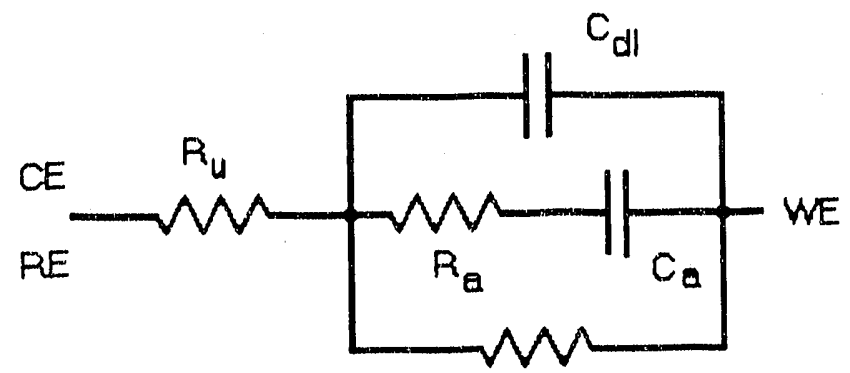

$R_{\text {ct }}$

In order to interpret the admittance data, the various elements of the circuit above are linked to physically relevant processes at the interface. The series resistive term $R_{u}$ corresponds to the "uncompensated" resistance in the capillary to charge flow. The interface itself is modeled by three parallel processes; charging the electrical double layer at the solid/liquid interface $\left(C_{d l}\right)$, adsorption of a secies at the intorface accompanied by charge transfer $\left(R_{a}, C_{a}\right)$, and net charge transfer by chemical reaction (Rct). Table 2 shows best fit quantities to most of these values obtained over the temperature range studied. The data in the table were determined by fitting the data based on analytical response of the model circuit. The high frequency limit of the admittance allows quick determination of the 


\begin{tabular}{|c|c|c|c|c|}
\hline$T /{ }^{\circ} \mathrm{C}$ & $R_{\mathrm{u}} / \Omega$ & $\mathrm{R}_{\mathrm{a}} / \Omega$ & $\mathrm{c}_{\mathrm{a}} / \mathrm{nF}$ & $\mathrm{c}_{\mathrm{dl}} / \mathrm{nF}$ \\
\hline 0 & $1.132 \times 10^{5}$ & $3.98 \times 10^{5}$ & 1.256 & 0.593 \\
\hline 5 & $1.121 \times 10^{5}$ & $4.06 \times 10^{5}$ & 1.118 & 0.745 \\
\hline 10 & $1.115 \times 10^{5}$ & $4.46 \times 10^{5}$ & 1.117 & 0.912 \\
\hline 15 & $1.114 \times 10^{5}$ & $4.93 \times 10^{5}$ & 1.020 & 1.102 \\
\hline 20 & $1.113 \times 10^{5}$ & $5.74 \times 10^{5}$ & 0.931 & 1.322 \\
\hline 25 & $1.110 \times 10^{5}$ & $7.36 \times 10^{5}$ & 0.836 & 1.556 \\
\hline 30 & $1.102 \times 10^{5}$ & $1.00 \times 10^{6}$ & 0.756 & 1.779 \\
\hline 35 & $1.099 \times 10^{5}$ & $1.97 \times 10^{6}$ & 0.808 & 2.064 \\
\hline 40 & $1.092 \times 10^{5}$ & $2.52 \times 10^{6}$ & 0.872 & 2.372 \\
\hline 45 & $1.082 \times 10^{5}$ & $2.33 \times 10^{6}$ & 1.230 & 2.612 \\
\hline
\end{tabular}

Table 2. model circuit values from admittance experimenta on an inert silver interface, perturbation magnitude $33 \mu \mathrm{v}$, overpotential $-4 \mathrm{mv}$.

series resistance because it is the dominating circuit element. The rolloff point and frequency at phase shift of $45^{\circ}$ provide estimates for $c_{d l}$. The low frequency portion of the admittance can be fitted to a sum of $c_{d l}$ and $c_{a}$.

From these starting estimates, the experimental data were fit by a graphical, iterative process. The uncertainty in the $R_{u}$ values were fairly large due to the instrumental distortion and corrections applied. The error here is a systematic shift rather than a precision error between the results at individual temperatures. The largest uncertainty in the determined values above is found in the $R_{a}$ values. These nimbers are valid to one figure since their impact on the observed admittance response was small. The $R_{C t}$ values were all quite large yet detectable 
through the low frequency limit of the phase angle in the admittance spectra. Limits can be placed on the maximum $R_{\text {ct }}$ value based on the steady state potential increase experiments. We estimate $R_{c t} \leq 2.5 \times$

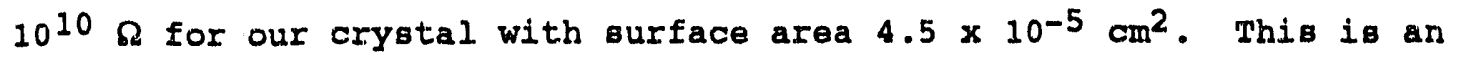
exchange current that is 10 orders of magnitude smaller than a typical polycrystalline electrode. The uncompensated resistance term varied consistently with temperature. Converting the values to concentrations revealed values consistent with the molar conductivities of $\mathrm{Ag}^{+}$and $\mathrm{NO}_{3}{ }^{-"}$. Solution diffusion coefficients from these conductivity values were on the order of $10^{-5} \mathrm{~cm}^{2} / \mathrm{s}$.

Measurements of the double iayer capacitance $\left(c_{d l}\right)$ are usually interpreted in terms of a series of three components 31 . These components are the electronic and ionic contributions $\left(C_{e l}\right)$, the contribution from rearranging water dipoles at the interface $\left(C_{D}\right)$, and the diffuse layer contribution $\left(C_{\text {diff }}\right)$. In these concentrated electrolyte solutions, the capacitance for the diffuse layer is effectively infinite. Our experiments are done on a silver surface around the equilibrium reduction potential, placing the surface at a potential very positive with respect to the point of zero charge. In this case, the capacitance from the electronic and ionic contributions should be much larger that the capacitance from dipole reorientation. Therefore, a series combination of these three components is dominated by the capacitance caused by the water dipoles:

$$
c_{d I}^{-1}=c_{e}^{-1}+c_{D}^{-1}+c_{d i f}^{-1} \cong c_{D}^{-1}
$$


In light of this, our results show the expected response with temperature, namely, the capacitance $c_{d l}$ increased with increasing temperature. Measurement of the dipole contribution to the interfacial impedance has historically been hindered by the presence of other faradaic processes (usually ret charge transfer). These inert interfaces provide negligible faradaic reactions at high surface charge, creating ideal conditions for measurement of $C_{D}$. Previous direct measurements of this quantity on low-defect structureless metals has been restricted to the liquid metals ${ }^{35}$.

A simple relation correlates the dipole capacitance per unit area to the effective dielectric function of the interface:

$$
\varepsilon=\frac{C_{D^{g}}}{A \varepsilon_{0}}
$$

This is the result for a parallel plate capacitor with thickness 8 and area $A ; \varepsilon_{0}$ is the permittivity of free space $\left(8.85 \times 10^{-12} \mathrm{~F} / \mathrm{m}\right)$. Since the closest approach of ions sets the boundary of the dipole capacitance region, the hard aphere diameter of water is a good approximation to $\mathrm{s}$ A plot of the temperature dependance of this quantity is shown in illustration 13. The dielectric constant of the interface at low temperature is very low in comparison to bulk water. This value approaches the limit of the polarizability of a "frozen" water molecule at the interface. At room temperature, the experimental dielectric constant is 10 while the bulk value is 78 (ref. 36). Our data suggest that the reorientation of the first layer is an activated process. The enthalpy of reorientation we measure is $\Delta H=24.5 \mathrm{~kJ} / \mathrm{mol}$. This term should reflect a combination of water-water molecule interactions 
through hydrogen bonding and water-metal bonding. The majority of this reorientation energy is from this second term.

The adsorption of apecies at the surface was included in the model in order to obtain the best fit to the admittance spectra. We assign the adsorption parameters extracted from the data to the adsorption of charge neutral silver adatoms. These adatoms would be free to move along the interface and desorb with accompanying charge transfer. Changes in surface concentration of these species due to a perturbation in potential appear as a capacitance $c_{a}$ and thei: rate of formation will produce a resistance $R_{a}$ in series with this capacitance. For full charge transfer (one electron lost per adsorbed atom), the surface coverage of silver adatoms $\Gamma_{A g}$ is related to the capacitance by;

$$
\Gamma_{\mathrm{Ag}^{0}}=\frac{\operatorname{RTC}_{\mathrm{a}}}{\mathrm{AF}}
$$

near the equilibrium potential. $R$ and $T$ have their usual meanings, $A$ is the surface area, $F$ is raraday's constant and the units of $\Gamma_{A g}$ are mol $/ \mathrm{cm}^{2}$. A plot of the natural logarithm of this quantity against $1 / T$ is shown in illustration 14 .

At the higher temperatures, the fit.s to the data were not as strongly dependant on the adsorption components, producing less confidence in the values from $35^{\circ} \mathrm{C}$ to $45^{\circ} \mathrm{C}$. The coverage of the species on the surface is fairly low at room temperature, $\theta=2.7 \times 10^{-3}$, or one adatom per 400 surface atoms. The adspecies formation energy over low temperatures is $\Delta B=-8 \mathrm{~kJ} / \mathrm{mol}$. For a ailver adatom, this energy 
reflects the both the loss of solvation, and stabilization by surfaceadatom bonding. The reaction of adatom formation as it is discussed here is different from the bulk deposition of Bilver, which may or may not be preceded by the formation of a silver adatom. The physical difference between deposition and adsorption at the interfacis is that deposition of an atom always results in a site for potential future growth. When a species adsorbs at a surface or interface, an adsorption site is occupied and will not be released to another species until the adrorbing species deposits or desorbs.

Our experimental study of the interfacial impedance of these inert interfaces represents the application of traditional impedance characterization to a new type of system. There has been previous impedance characterization experiments conducted on si.lver electrodes, however they were conducted at only at room temperature and in the presence of large faradaic currents 15,17,19. This now, inert, eystem has allowed some modification of the typical approach. Dive to the negligible net faradaic reaction of growth at the surface, we were able to measure the interfacial admittance over several decades of frequency. This wide frequency range of data increases our confidence in the ability of the model circuit to describe the relevant processes at the interface. In making conclusions regarding dipolar orientation energy and ilver adatom formation we had to assume that their signatures were appearing in $C_{D}$ and $C_{a}$ respectively. However, these are the most aensible assignments based on the physics and chemistry of the surface under study. 
When active, the silver ourfaces demonstrated a range of response cheracteristics depending upon cell potential, cell temperature and surface morphology. The most interesting and tractable results we observed in the growth of these silver surfaces were when the growth shifted from regular growth (amall normalized deviation of current) to periodic growth (large normalized deviations). Interpretation of the net current flowing through the cell is simple; it is always related to a reaction rate multiplied by an active site number. complexity appears for systems which have a number of site clasees, each with a different rate of reaction. In general, the growth current can be expressed as;

$$
i \propto \sum_{j} k_{j} N_{j}
$$

with $k_{j}$ being the rate of resction and $N_{j}$ being the number of sites of a particular class, j. This general expression describes typical growth, a sum of a number of microscopic processes producing a total reaction rate which is an average of the individual rates weighted by the number density of sites. Since a large number of sites can contribute to tho growth, the observed current is regular and has emall fluctuations. When a limited number of classes are involved in growth, it is feasible to correlate the instantaneous current with the microscopic rate of reaction $k_{j}$. For example, the growth of a aingle isolated twodimensional cluster will produce a growth surrent proportional to the peripheral length of the cluater. The periphery of the cluster provides the potential future growth bites, so as the cluster expands, the reaction rate increases; 
$i \propto \mathrm{knl} \quad(I=2 \pi r$ for a circular growth front $)$

where $I$ is the peripheral length and $n$ is the number of active growth sites per unit length of periphery. Previous work has demonstrated the utility of such an interpretation 26 . Essentially, the current resulting from a simple growth event can be used to define the geometric constraints involved in the event. We were able to observe growth events that corresponded to a fraction of a monolayer in total coverage (1 $\times 10^{9}$ atoms), describing activity on a subset of the entire surface within the capillary. From a simple geometric interpretation of equation 7, we were able to determine the time dependent peripheral length from the current for a constant $k$ and locate the site of initial cluster formation.

A typical growth event which we observed is shown in illustration 15. Each growth event begins with a linear region of growth. The alope of this region io identical to other growth events appearing in the same experiment. This portion of the growth corresponds to a cluster expanding freely on the surface. In our experiments, the growing cluaters are bounded on one side by the edge of the cryatal next to the capillary wall. Illustration 9 shows the configuration; the cluster begins growth at nucleation site $N$ and expands with rate $v(v=k n)$. When this expanding layer strikes the edge of the facet, the active peripheral length decreases as long as spillover onto the other facet does not occur. Since the cluster's peripheral length is decreasing, the current flowing through the cell drops while the expanding layer 
completely covers the facet. The solid line in illustration 15 is a best fit model curve based on this simple geometric interpretation, assuming geometry as in illustration 9. The cluster appears to grow as circle, under kinetic rather than thermodynamic control. From the growth events, the isotropic rate of expansion $v$ is determined from the initial linear region. The distance $h$ in illustration 9 is calculated in two ways; from the capillary radius and the charge passed in the growth event and from the time required for the clubter to reach the facet edge at growth velocity v. This provided a self-consistency check of the geometric interpretation. For a given transient, the agreement in these factorn was good to a few hundred nanometers (about 58 of the total distance, $h$ ). The origin of the cluster is determined as a sift from a mirror plane of symetry by the fit of the moded to the tail of the transient. A self-consistency check can also be applied to this location by interpretation of a Beries of experiments and will be described later in this chapter. of courbe, this geometric interpretation was selected from many posibilitiea. In fact, model curves for a number of possibilities were calculated, one section of chapter 2 presents a very important example of the growth of a circle into an elliptical boundary. Along with the consideration of other possible models, extensive data from growth events of slightly varying geometries were applied in the determination of the best description of the surface morphology. A longer portion of the time series which contains the data in illustration 15 is presented in illustration 16. During this experiment, sets of small events are separated by large events. Each of these large events amounts to the 
passage of several monolayers of surface charge. The large events also produced changes in the size of the surfuce region active in the small growth events. From these data, a model of the surface was constructed, a pictorial representation is in illustration 17 . The side view in this illustration shows in the shaded region the material added in a large growth event and its effect on the length $h$. Optical observations of silver crystals growing in capillaries confixmed the presence of this type of surface morphology. In illustration 18 the small events appear in a regular fashion. overall, we observed this type of behavior guite frequently even though it has not been discussed in previous work in this system. Previous analyses of microscopic growth events on silver. surfaces within capillaries have described eventa which appear as a stochastic time series. The events in those previous otudies 26,27 were stochastic in both arrival time between events and in the apparent location of the nucleation events. The presentation of stochastic observations was used to establish that the ourfaces were defect free. on the other hand, we find that the surfaces prepared in capillaries contain lower defect densities than free standing single crystals yet are far from defect free. A signature of the presence of interfacial defects are temporally and spatially deterministic events as in illustration 18 . In fitting sets of transients like illustration 15 we observed two important points. Firstly, all of the nucleation events occurred at approximately the same location. Secondly, when observing several microfacet sizes, $h$, the time between growth events varied in a regular manner. These two observations of periodic growth represent significant departures from the reported behavior of this system. 
Illustration 19 Bhows a sumary of the fitted results over several minutes of observation. The values for each $h$ and $\tau$ (waiting time) are calculated by averaging the fitted values for each set of small events. The waiting time $\tau$ was extracted from the growth events by moasuring the time between the peak of a growth event and the time to the start of the next growth event. The data in this illustration are from the $20^{\circ} \mathrm{C}$ data, the data acquired at $50^{\circ}$ also showed this linear behavior. The strong linear relation between facet length and square root of time implies a relation of the form:

$$
h^{2}=c \tau
$$

This is the expected result when transport via surface diffusion is the rate determining step in the nucleation process! The diffusion length here is identical to the length from facet edge to nucleation site. At very short times, the intercept of the plot is another measure of the location of the active nucleation site. This was an unexpected result since the defect site that catalyzes the formation of critical clusters appears to rely upon the arrival of surface species in the initial stages of cluster growth. In sumary, these experiments observe nucleation at a single aite within our ability to locate it via geometric analyois, and the arrival times of these nucleation ovents appear to be related to surface transport.

The combination of two non-linear processes, mass transport and nucleation, have produced another chemical oscillator ${ }^{37}$. Solution diffusion of reactants has been eliminated as candidate for the 
limiting mass transport mechanism by the high concentration of silver ions and their mobility. A fluctuation in the local concentration created by the oudden passage of the charge in the transients would be replenished in a few milliseconds. We turn to surface-based mass transport to provide the reactants for a nucleation event. Other aurface regions should have some oquilibrium coverage of adsorbed silver atoms as measured by the interfacial impedance studies. We also know from those studies that the rate of formation of the adsorbed species is very alow. Using the impedance results for $C_{a}$ and $R_{a}$ at $20^{\circ} \mathrm{C}$, the rate of adatom formation is so slow that an equilibrium concentration on a amall, narrow surface region could be established faster by diffusion from a nearby large source.

To convert the observed slopes in the $h \mathrm{vs} . \tau^{1 / 2}$ plots into a surface diffusion coefficient, the experiment was computationally modeled. The model details and an extensive discussion of the calculation are in chapter 2. The simple diffusion equation 38 ;

$$
\left\langle x^{2}\right\rangle=2 D_{B} t
$$

could not be applied to the observed results because this system is driven by a concentration gradient. Each layer that grows across the interface depletes the surface concentration of adsorbed ailver atoms. Diffusion from the facet edge begins when the new layer comes in contact with the bounding edge. The computational model treated the "rest of the crystal" as a source of surface concentration as determined by the interfacial impedance work. After each growth event, hypothetical 
adatoms were allowed to diffuse from the equilibrium source until they were detected at the nucleation site. The microfacet region constructed a "time-of-flight" experiment where adatoms cepart from a source, diffuse across a region of known length, and are detected at a sink by the growth event which they cause.

The simulations provided correction factors that were used to convert the linear relation between the region length and the diffusion time to a diffusion coefficient of the usual sense. Table 3 presents our results and a comparison to two previous molecular dynamics calculations for a close-packed fcc metal surface in contact with a vacuum 39,40 . For silver, previously available techniques for measuring metal surface self-diffusion coefficients (e.g. field emission microscopy 41,42 , have not been practical. This is the first direct measurement of a self-diffusion coefficient for the surface of silver exposed to any medium. It is also the first direct measurement of diffusion of metal atoms at the metal/water interface.

\begin{tabular}{|l|l|l|l|l|}
\hline System & $D_{0} / 10^{-4}$ & $E_{\mathrm{a}} /$ & $D_{\mathrm{B}} /\left(20^{\circ} \mathrm{C}\right)$ & $D_{\mathrm{g}} /\left(50^{\circ} \mathrm{C}\right)$ \\
& $\mathrm{Cm}^{2} \mathrm{~s}^{-1}$ & $\mathrm{~kJ} \mathrm{~mol}-1$ & $10^{-7} \mathrm{~cm}^{2} \mathrm{~g}^{-1}$ & $10^{-7} \mathrm{~cm}^{2} \mathrm{~g}^{-1}$ \\
\hline $\mathrm{Ag}(111) / \mathrm{B}_{2} \mathrm{O}$ & 2.7 & 19 & $(1.2 \pm 0.3)$ & $(2.5 \pm 0.9)$ \\
\hline $\mathrm{fcc}(111)$ & 3.4 & 12 & & \\
\hline $\mathrm{fcc}(111)$ & 5.3 & 13.3 & & \\
\hline
\end{tabular}

Table 3. Comparison of experimental results and molecular dynamics results scaled to silver surface self-diffusion.

For our two temperatures, it is possible to calculate an activation barrier and intercept for a thermally activated diffusion 
process;

$$
D=D_{0} \exp \left(\frac{-E \mathbf{a}}{\mathrm{RT}}\right)
$$

We calculate a significantly larger activation barrier than the molecular dynamics simulations. Since the molecular dynamics calculations have provided good agreement with other experiments on fcc close-packed surfaces in ultra-high vacuum, portion of the disagreement could be due to the role of liquid etructuring at the interface. From the interfacial impedance studies, we predict a moderate reorientation energy for the water dipole. The activation barrier to silver diffusion is a bit smaller, suggesting that along with the metal-metal interaction normally present, the water layer io slightly distorted by the passing adatom from its equilibrium position. This conclusion is consistent with work suggesting that structured water overlayers on metals are arranged in a loose hydrogen-bonded network 43. These experiments suggest that the presence of water at the surface mildly perturbs the energetics of normal surface diffusion. Additional results at other temperatures are not reported due to the difficulty of these experi lents. Additional experiments to measure surface diffusion around these temperatures would be nice, but their utility is limited oince the experimentally available window of $1 / T$ is narxow. For a narrow region of $1 / T$, the determined activation energy and intercept will always have a high uncertainty. 
The study of inert and slightly active surfaces allowed determination of quantities which have previously been experimentally problematic. We have observed the reorientation of water molecules through impedance spectroscopy. Nucleation rates for inert surfaces and the observed experimental results of multiple workers have been placed in a context of surface free energy terms and defect site stabilization factors. Finally, the impact of surface diffusion on microscopic growth processes ware observed. From temporal analysis of isolated growth events, surface diffusion coefficients for silver surface atoms were determined. One interesting direction for further study on this oystem would be the use of defect site location information of as a guide to the study of the defect surface structure. A map of several sites with different cluster nucleation rates could be used to correlate surface structure with energetics and reactivity. 


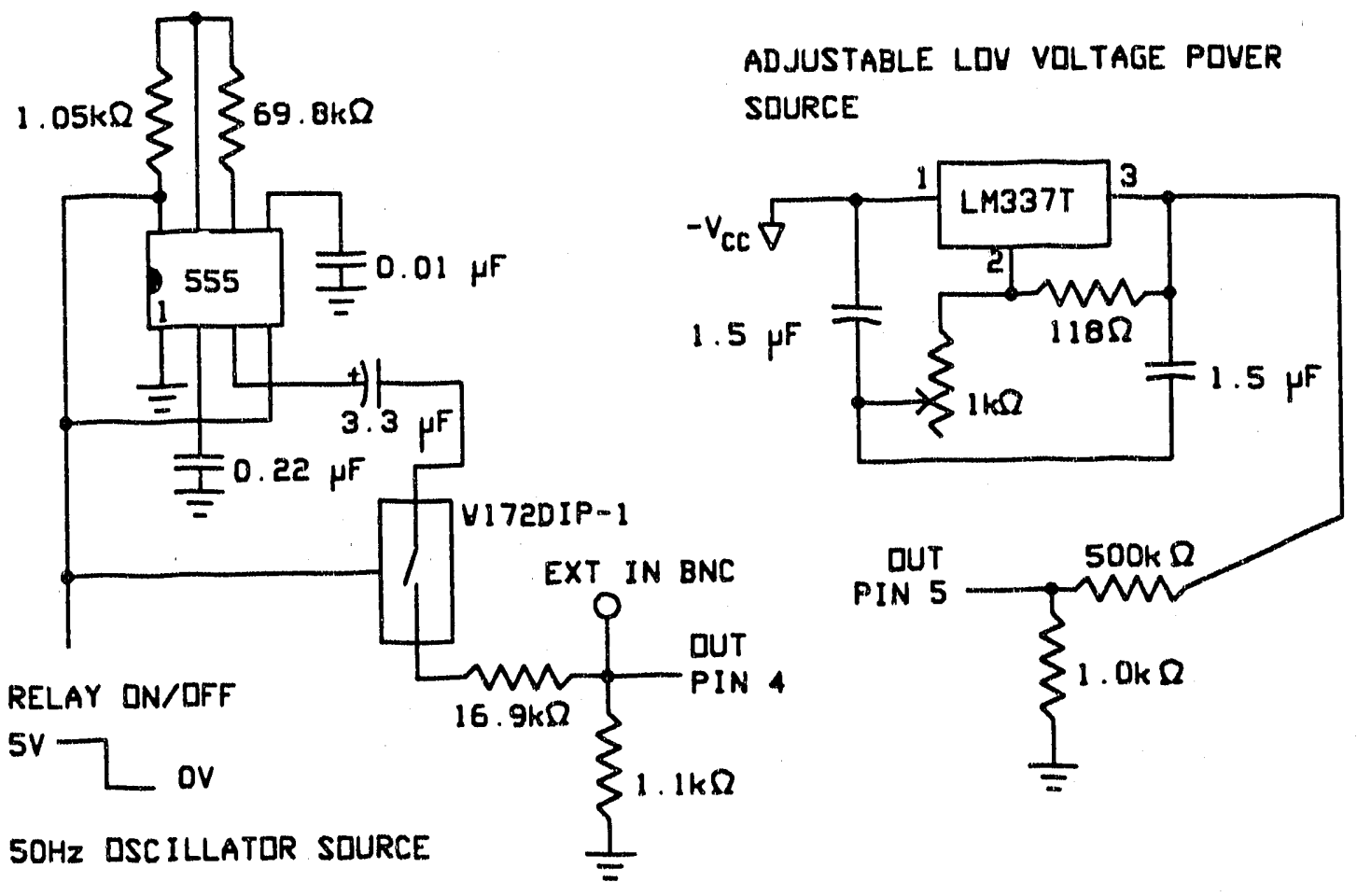

illustration 1:

Control aignal generation circuit. At left is the square-wave oscillator subsystem, to the right is the adjustable precision voltage circuit. 
×100 NON-INVERTING BUFFER

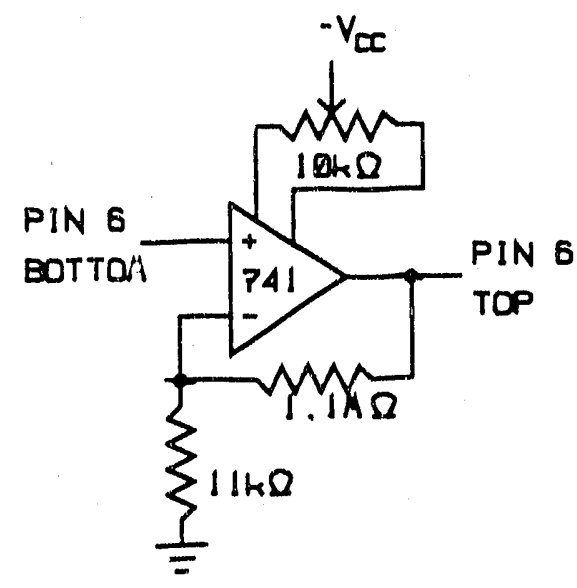

ADUSTABLE BANDWIDTH INVERTING BUFFER

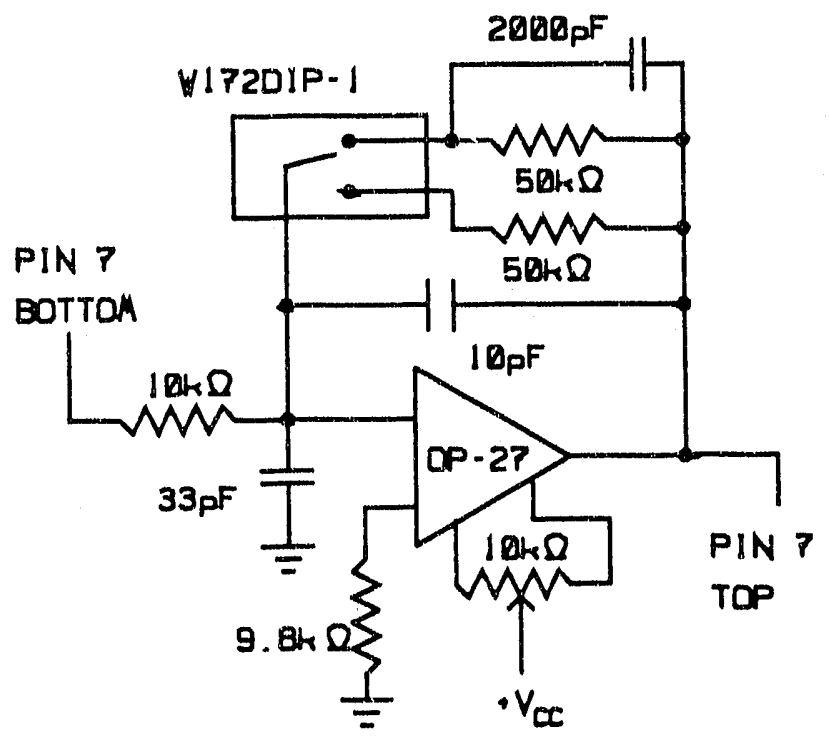

illustration 2:

Two buffer circuits. At left is a fixed gain non-inverting amplifier used to monitor the applied potential. The adjustable amplifier raises the current signal to high levels, and performs time averaging of the signal. 


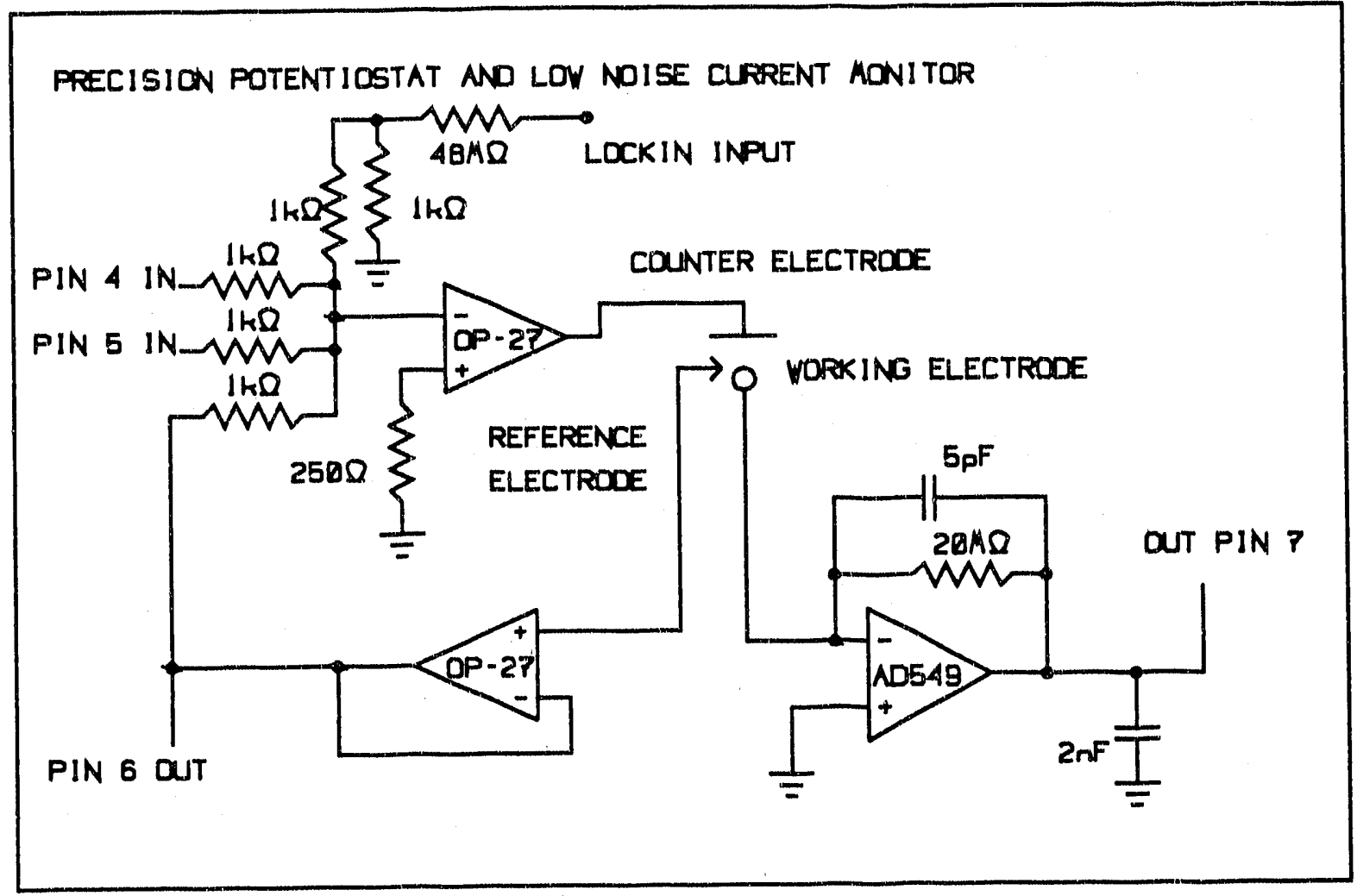

illustration 3:

circuit design for a simple three-electrode potentiostat. The useful current range goes down to $\mathrm{pA}$, with current gain at pin 7 of $20 \times 10^{6} \mathrm{~V} / \mathrm{A}$. 


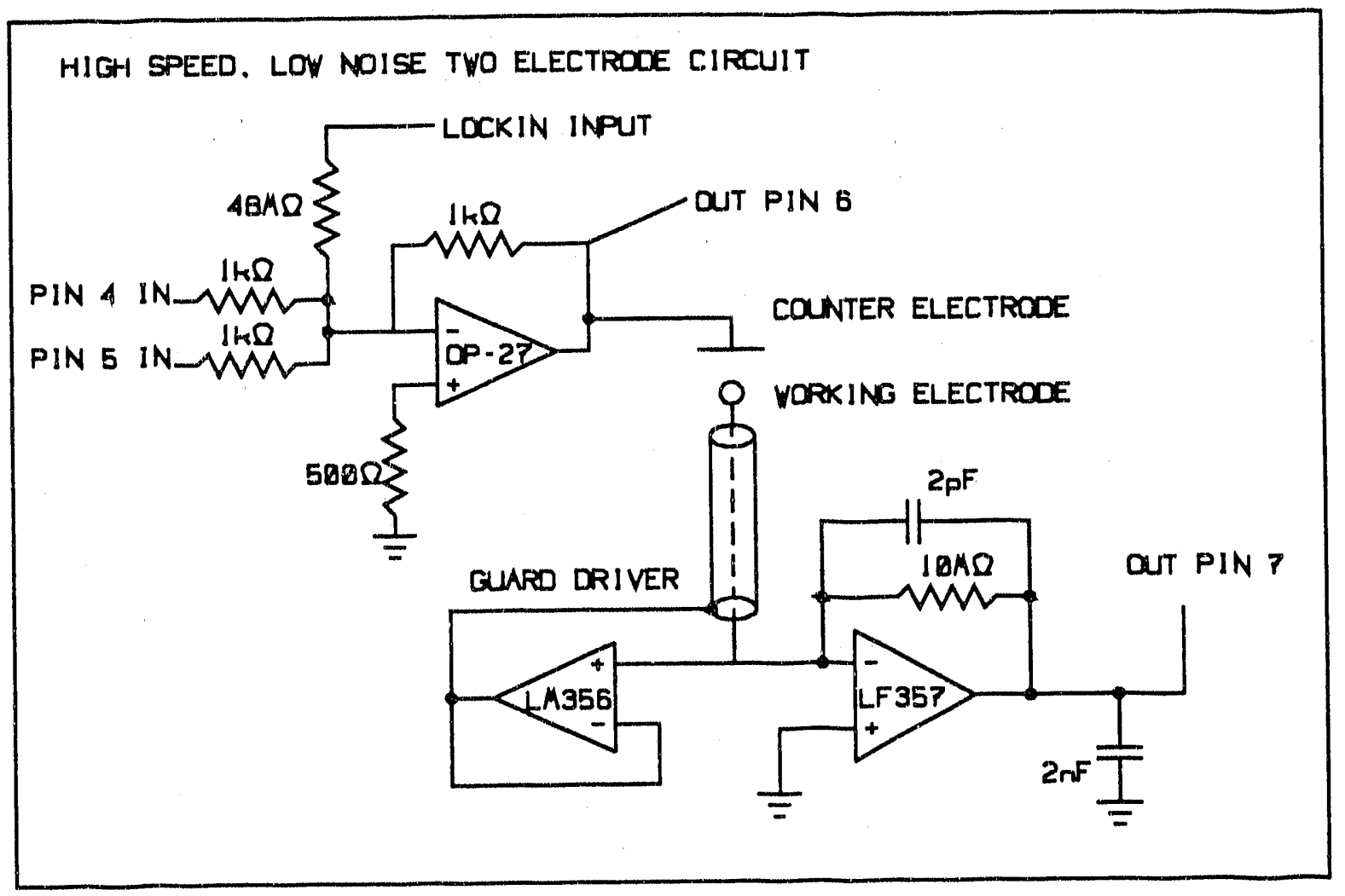

illustration 4:

Bigh-speed two-electrode electrochemical cell control circuit. The useful current range goes down to 100pA, with current gain at pin 7 of $10 \times 10^{6} \mathrm{~V} / \mathrm{A}$. 


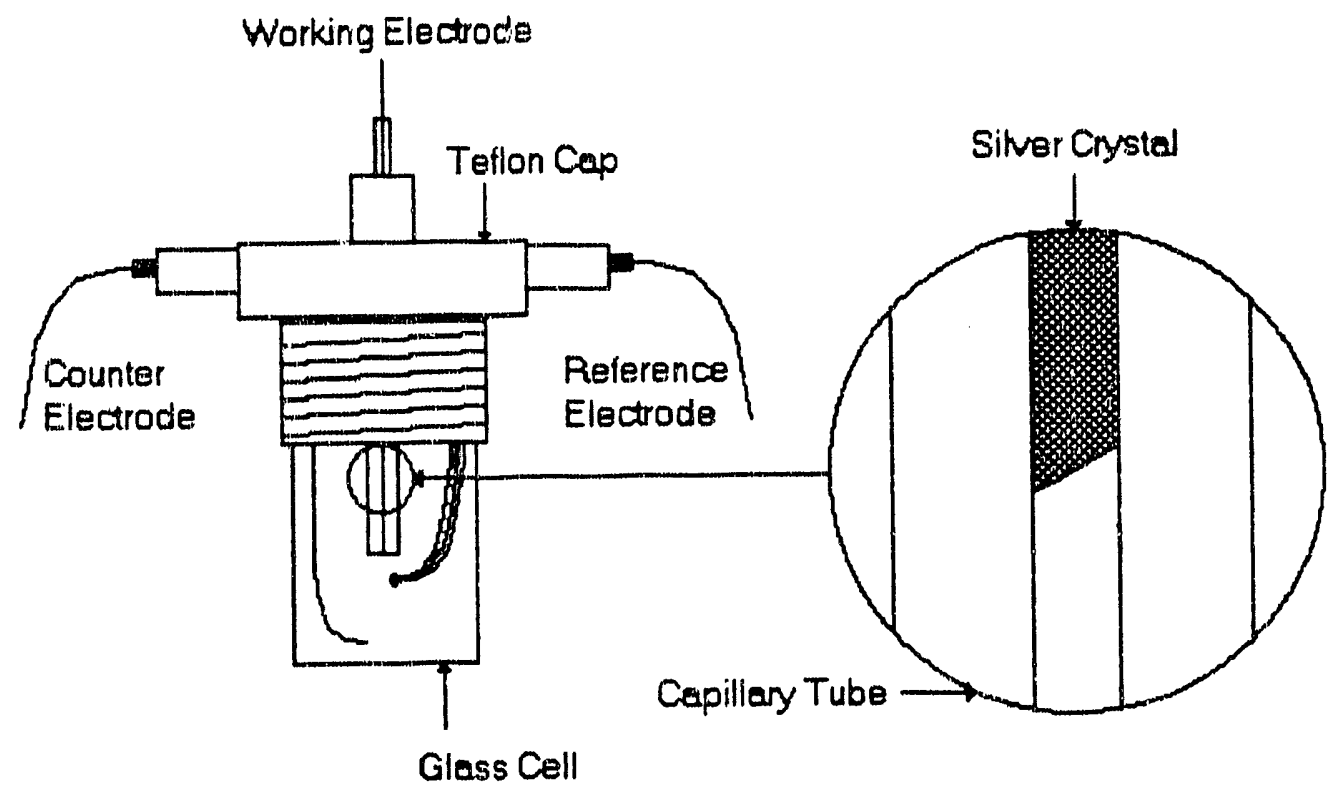

illustration 5:

sketch of experimental cell. The long cormercial capillary and sealed reference electrode are shown here. The exploded view showe the capillary bore and silver cryetal growing within. 


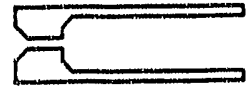

Initial Shepe

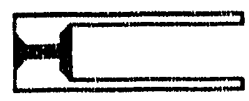

Filled with Wox

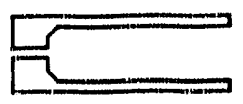

Final Shape

illustration 6 :

preparation of short capillaries. At left is the shape resulting from rotating within the torch flame. The eapillary is filled with black wax to protect the bore while polishing. The end is poliehed until a uniform diameter tube is present at the end of the capillary and then the wix is removad. 
Thermal Control Apparatus:

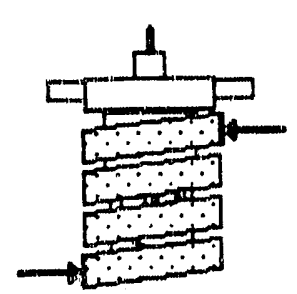

Copper tube coil

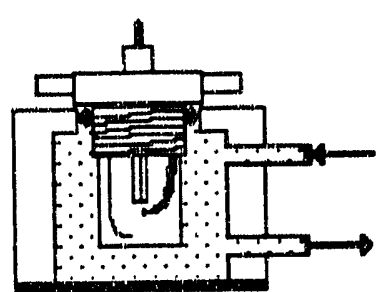

Plexiglass Jacket

\section{illustration 7:}

Two configurations used for thermal control. The copper coil was wrapped with cotton gauze for additional insulation. For both configurations, cotton gauze was placed over the top part of the cell. 


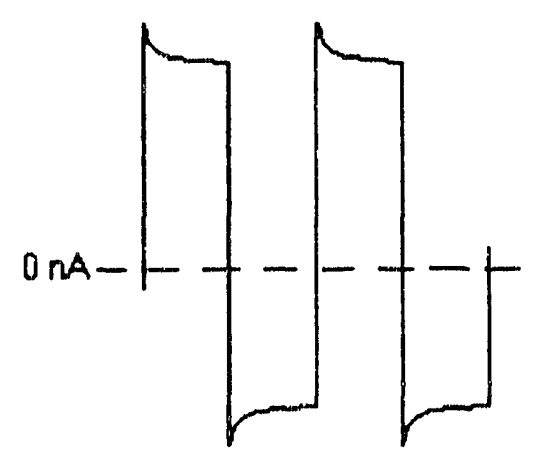

a) initial response

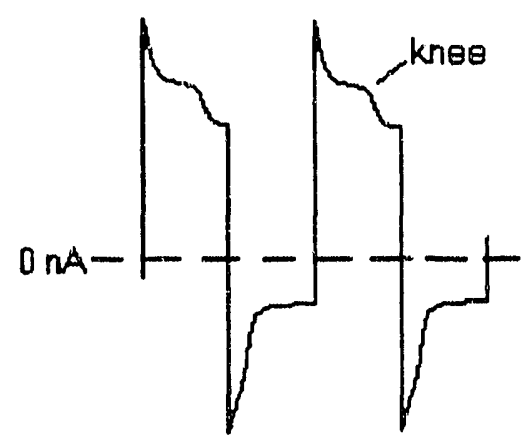

b) low reactivity surface

i.l.lustration 8:

sketches of oscilloscope traces observed during growth. A net overpotential towards growth produces the asymetry in the peak current values. The shape of the knee was exaggerated as the aurface raactivity decreased. 


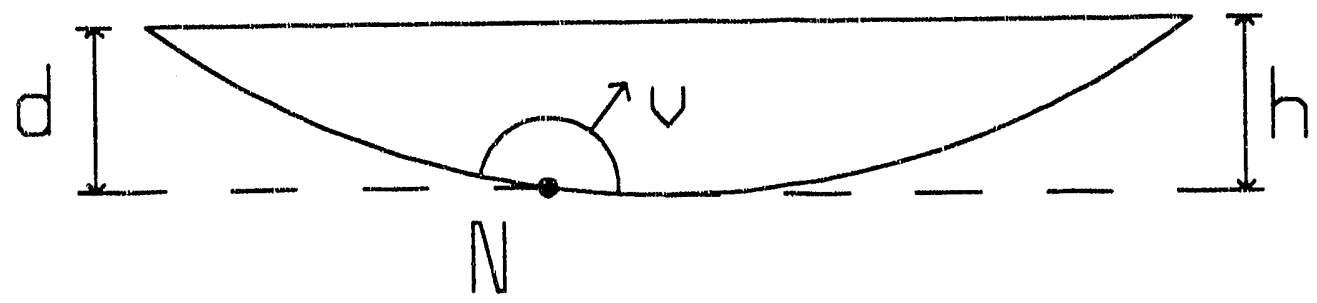

illustration 9:

Geometry of active portion of surface during a set of experiments at $20^{\circ} \mathrm{C}$. N denotes the site at which all growth events began. The distance of the microfacet edge to the active site is d while the maximum distance from the capillary wall to the microfacet edge is $\mathrm{h}$. Modeling of growth events as isotropically expanding circles allows calculation of the expansion rate, $v$ and the nucleation site $N$ from the experimental data. 


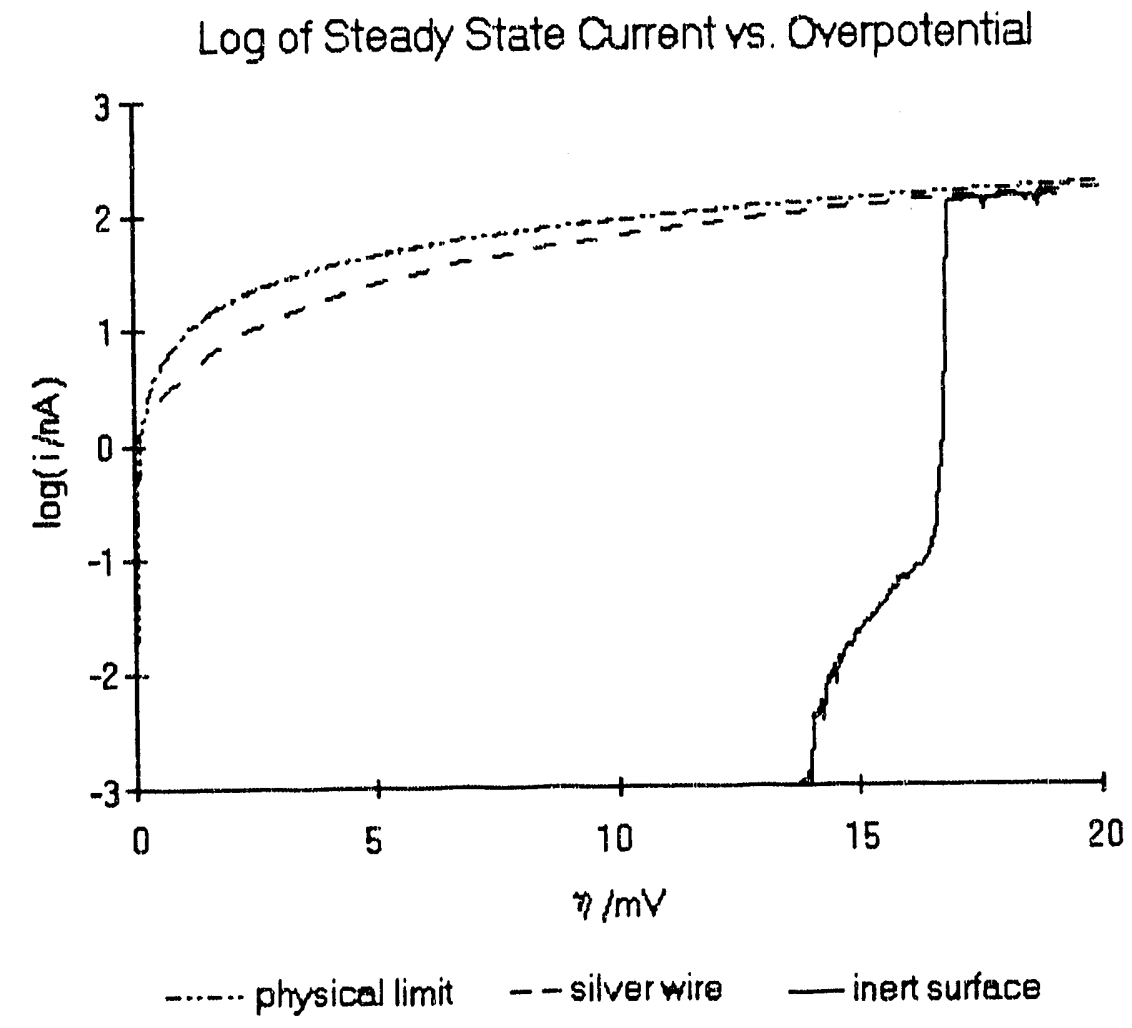

illustration 10:

Steady state current response at $45^{\circ} \mathrm{C}$ for a silver wire and an inert silver surface. The physical limit, ohrn' law $v=i R_{u}\left(R_{\mathrm{l}}=110 \mathrm{k} \Omega\right)$, is included for comparison. 
Log of Nucleation Rate vs. Overpotential

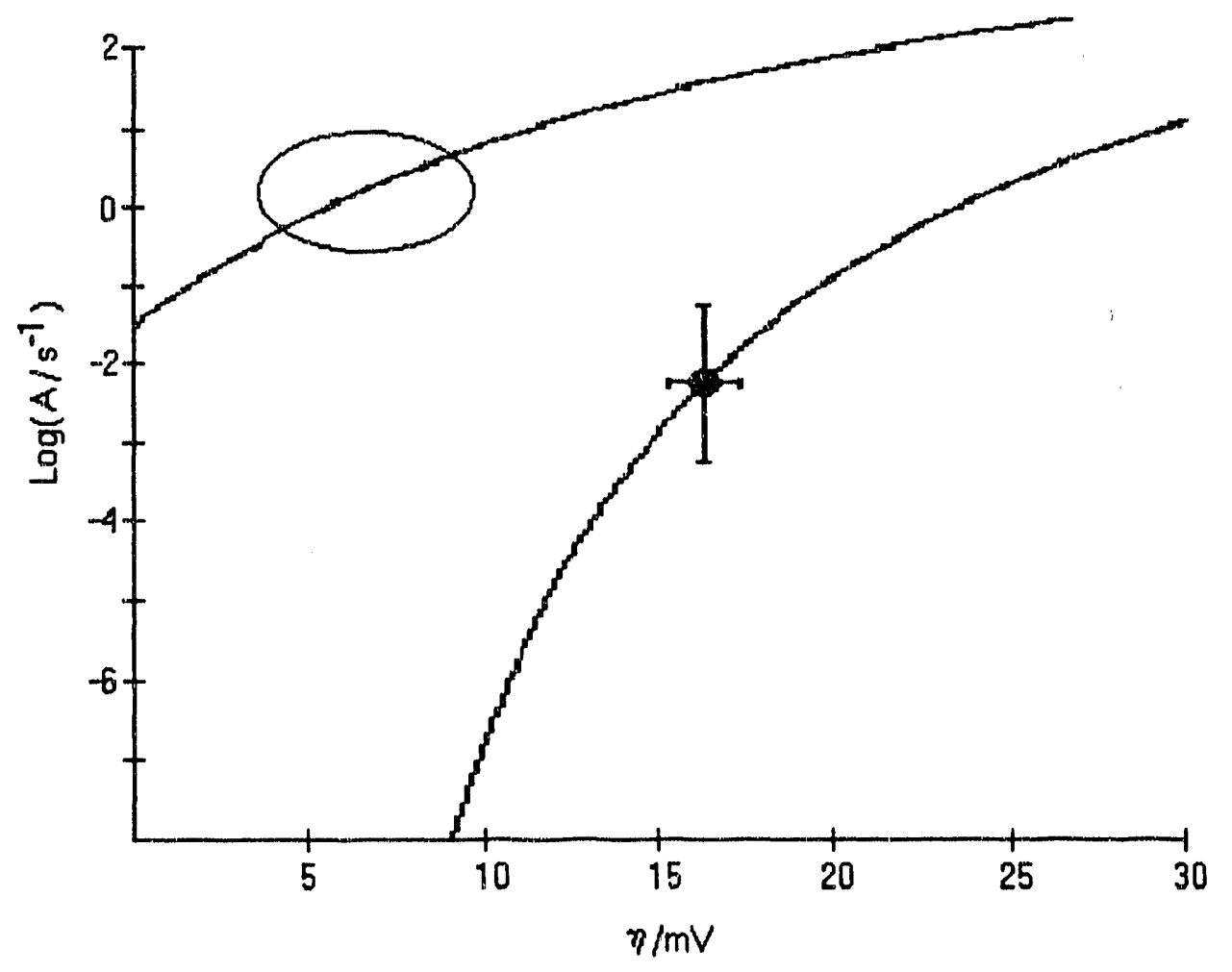

illustration 11:

Nucleation rate versus overpotential. Solid curves are models calculated at two stabilization factors $\sigma_{13}$. The experimental point with error bars is drawn from the data shown in illustration 10. The model curve through this point uses cluster edge energy $\varepsilon=2.7 \times 10^{-13}$ $\mathrm{J} / \mathrm{cm}$ and $\sigma_{13}=0$. The elliptical region at the upper left bounds previously reported random nucleation and growth events. The model curve through these data is calculated with cluster stabilization term $\sigma_{13}=-4 \times 10^{-2} \mathrm{~N} / \mathrm{m}$. 


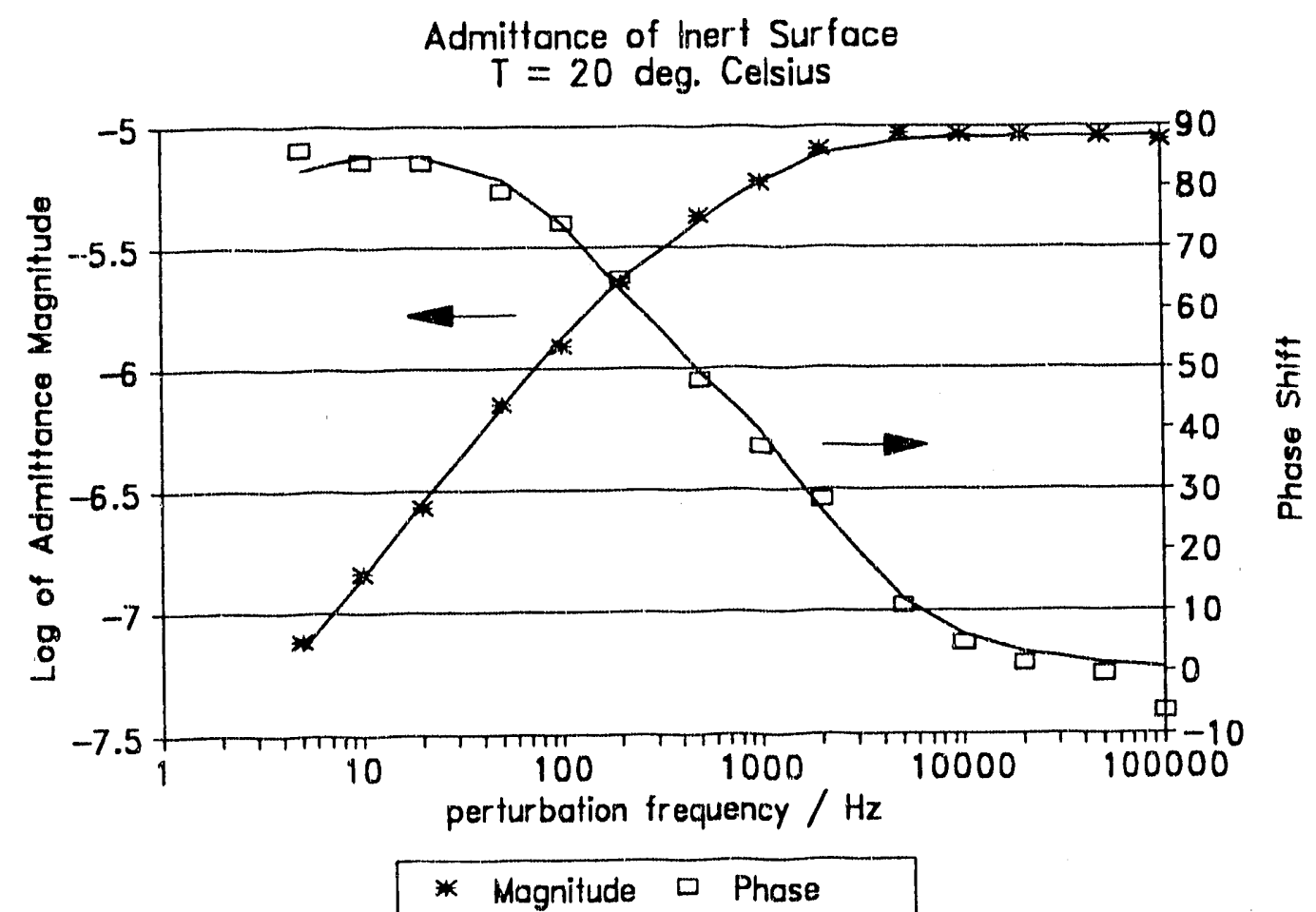

illustration 12 :

A plot of the admittance at one temperature and the best fit model curve. Cell admittance i.s the reciprocal of the cell impedance. The experimental data has been corrected for instrumental distortion as described in the text. Perturbation magnitude $33 \mu \mathrm{V}$ and applied potential $-4.0 \mathrm{mV}$. The low frequency limit illustrates the inert nature of the surface, the falling admittance magnitude indicates a negligible d.c. conductance. At high frequencies, the cell response is dominated by the capillary resistance $R_{u}$. 
Log Plot of Interfacial Dielectric

Constant vs. $1 / T$

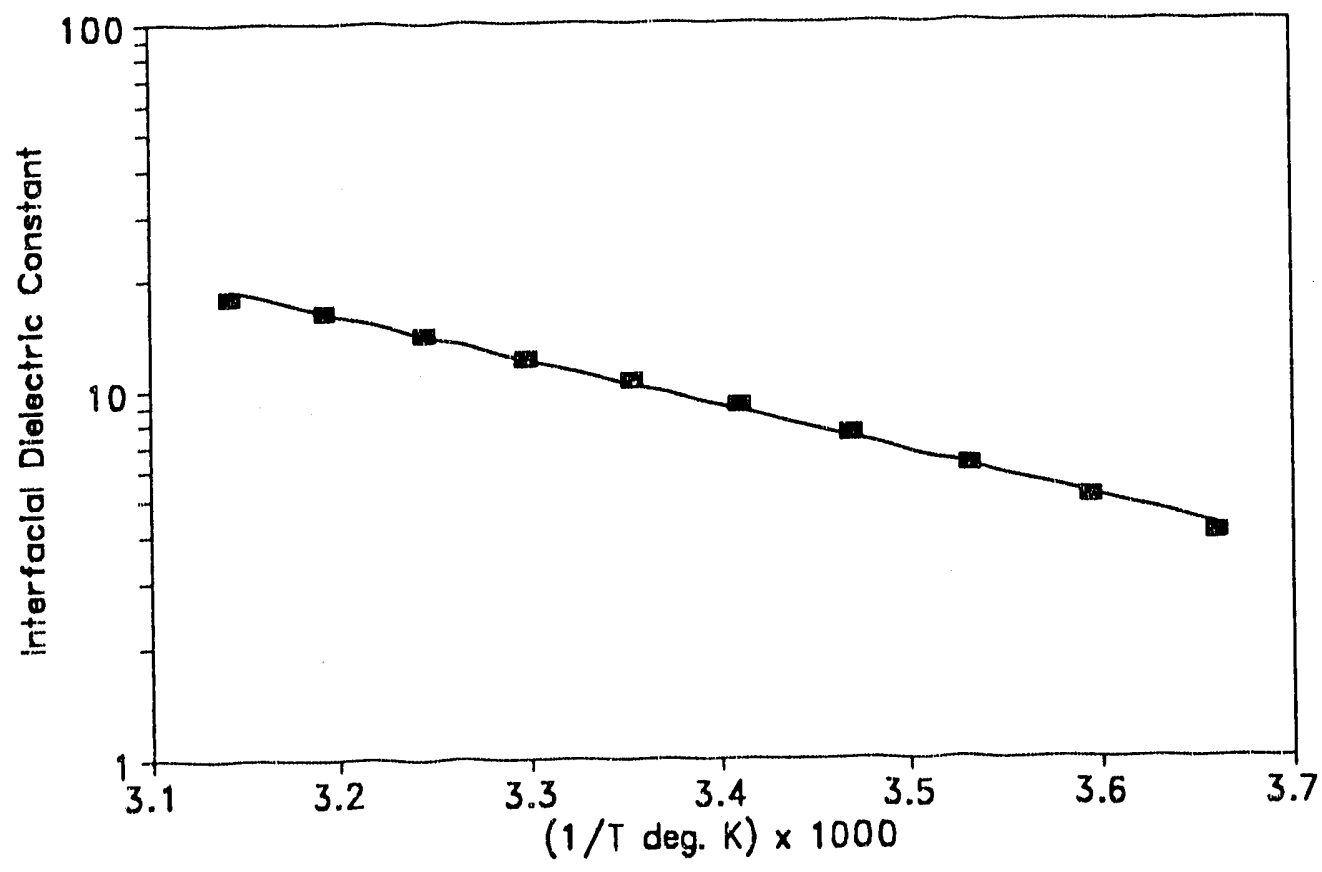

illustration 13:

Interfacial dielectric constant $(\varepsilon)$ determined from the double layer capacitance $c_{d l}$. Values assume a parallel plate capacitor with thickness of the water hard sphere raduis. This distance approximates the distance of closest approach of ions within solution. The interfacial dielectric constant at $25^{\circ} \mathrm{C}$ is significantly less than the bulk value of 78 . Least squares line shown through the data, $r^{2}=0.996$. 


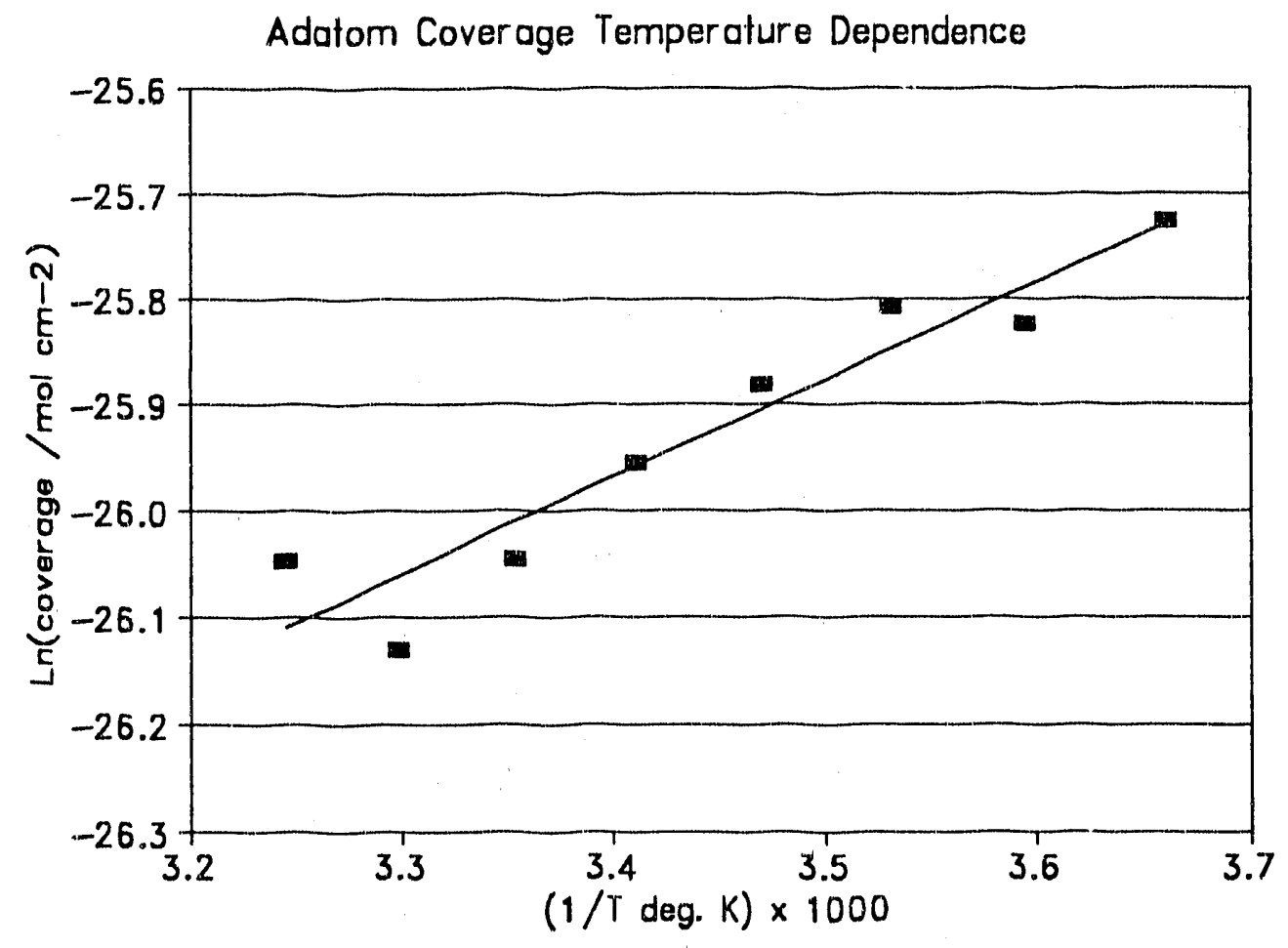

\section{illustration 14 :}

Adatom coverage determined from adsorption capacitance plotted against reciprocal temperature. High temperature results were hindered by signal resolution in the admittance data. Activation energy from lower temperature least squares fit is $\Delta \mathrm{\theta}=-7.6 \mathrm{~kJ} / \mathrm{mol}, \mathrm{r}^{2}=0.903$. 
Single Growth Event and Model Curve

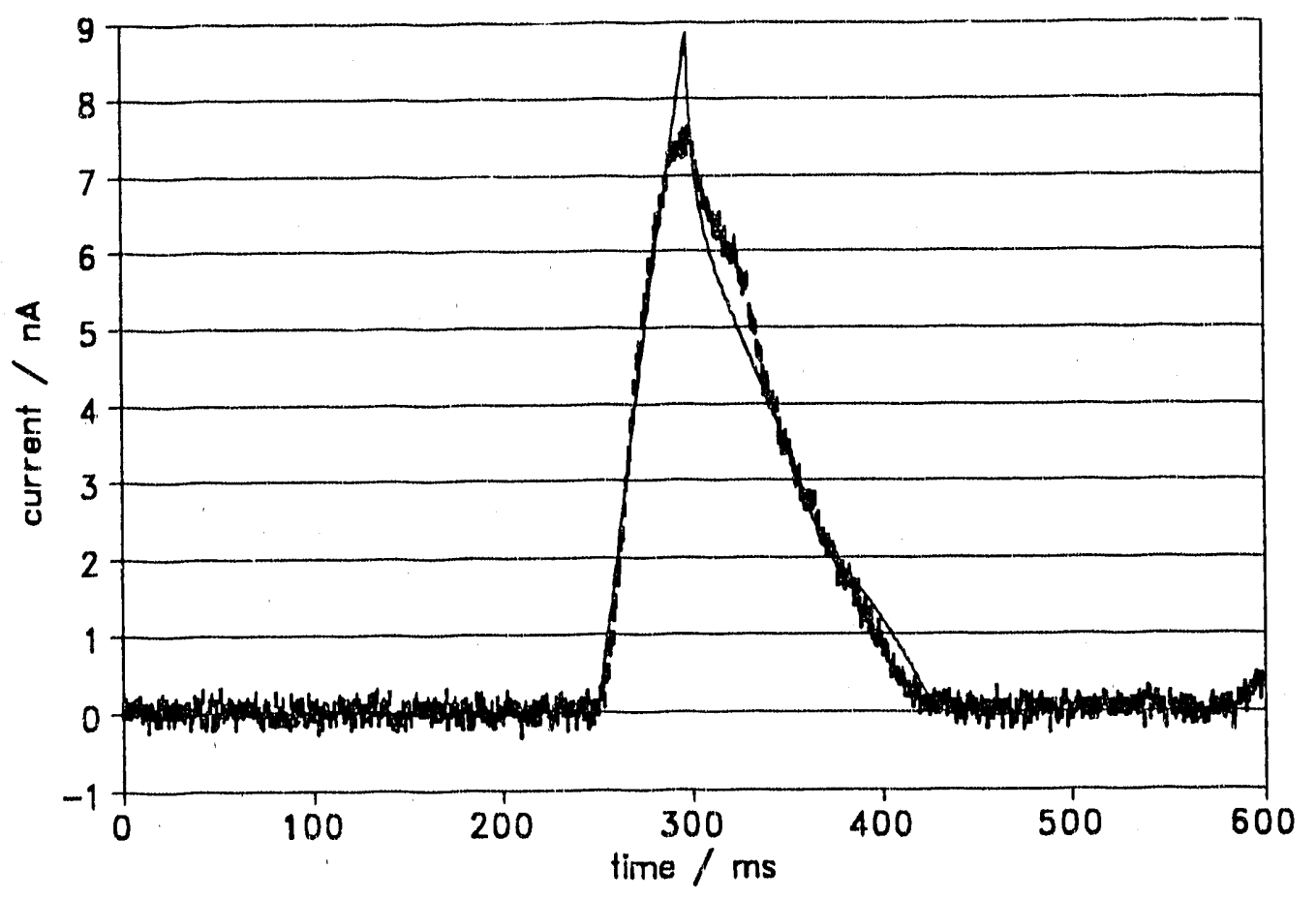

illustration 15 :

single growth event and beat fit model curve. Solution temperature $20^{\circ} \mathrm{C}$, overpotential $\eta=-1.0 \mathrm{mv}, 1 \mathrm{kHz}$ data sampling rate. Total growth charge $0.582 \mathrm{nc}$, expansion rate $\mathrm{v}=1.8 \times 10^{-2} \mathrm{~cm} \mathrm{~s}^{-1}$, microfacet size $h=8.2 \mu \mathrm{m}$, assuming Ag(111) surface packing density. Several of these small events were observed in a regular sequence. 


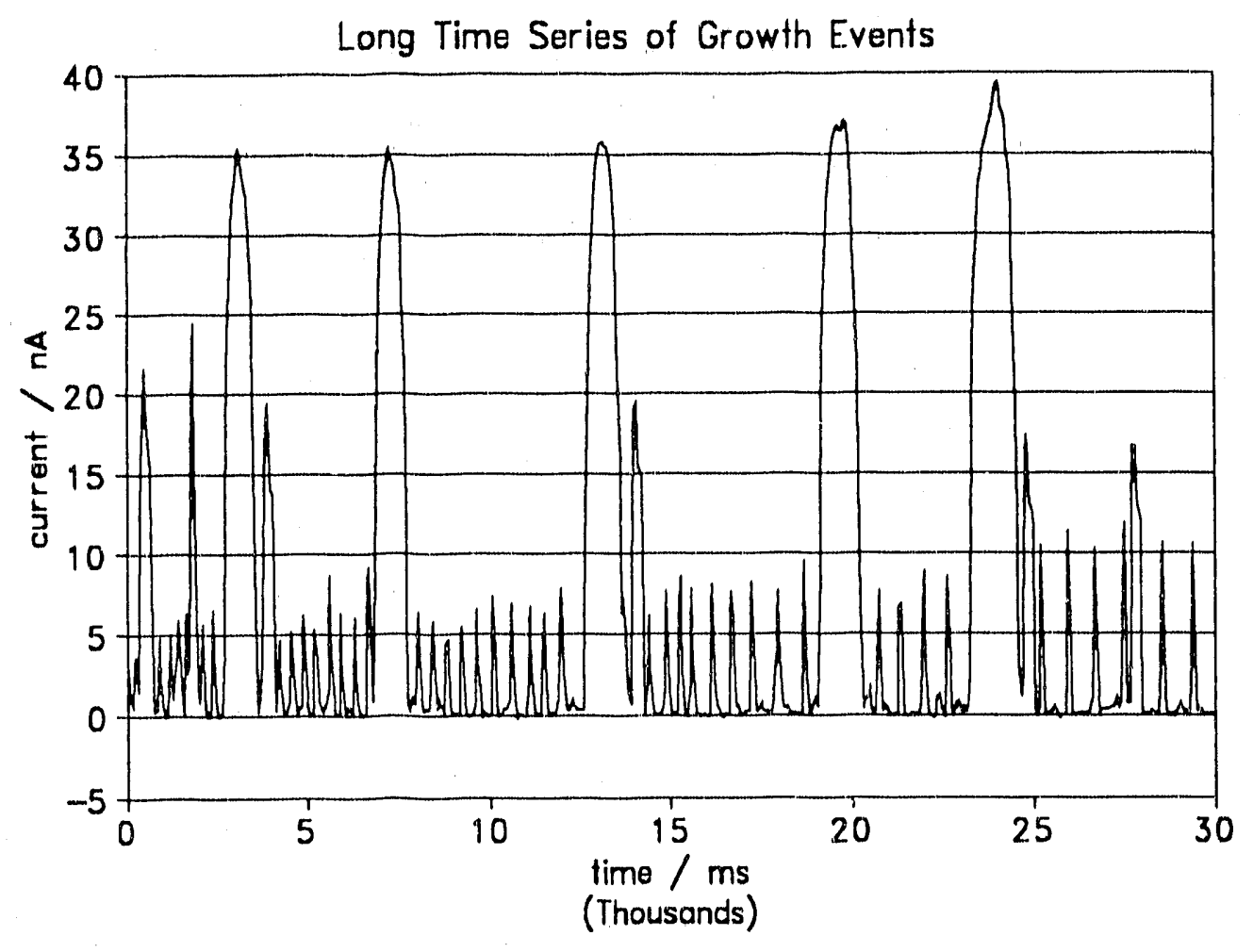

ilustration 16 :

Long time series, 30 seconds, showing a mix of large catastrophic events and the small regular events. The large events consist of 40 to 60 times greater charge passed than the small eventa. The period change between the regular events caused by the catastrophic events is quite clear. Growth conditions as in illustration 15. 


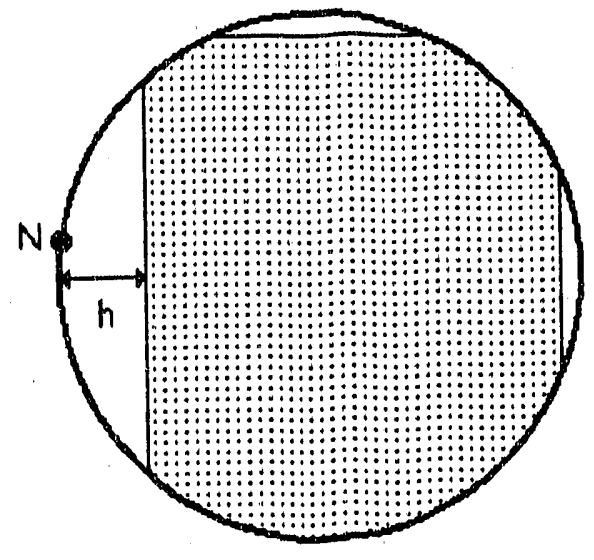

a) top view

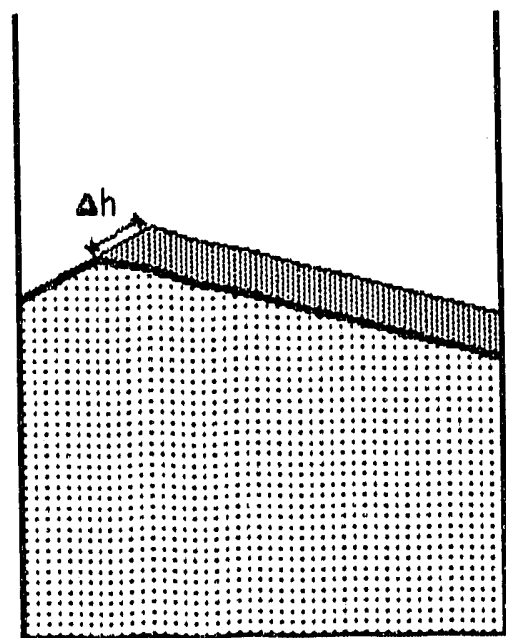

b) side view

\section{illustration 17:}

sketch of active surface inferred from the experimental data in illustration 16. A large Ag(100) crystal surface, Bquare grid, occuppies the major portion of the capillary. The sides bound omall facets which are bounded by the circular capillary boundary. One of these small (111) surfaces is the location of the emall regular eventi. For the data shown in illustration 15 , the nucleation site is denoted by $N$ in this figure. The side view shows a possible mechanism by which the catastrophic events can increase the microfacet size h. Scale exaggerated for clarity. 
Five Regularly Spaced Growth Events

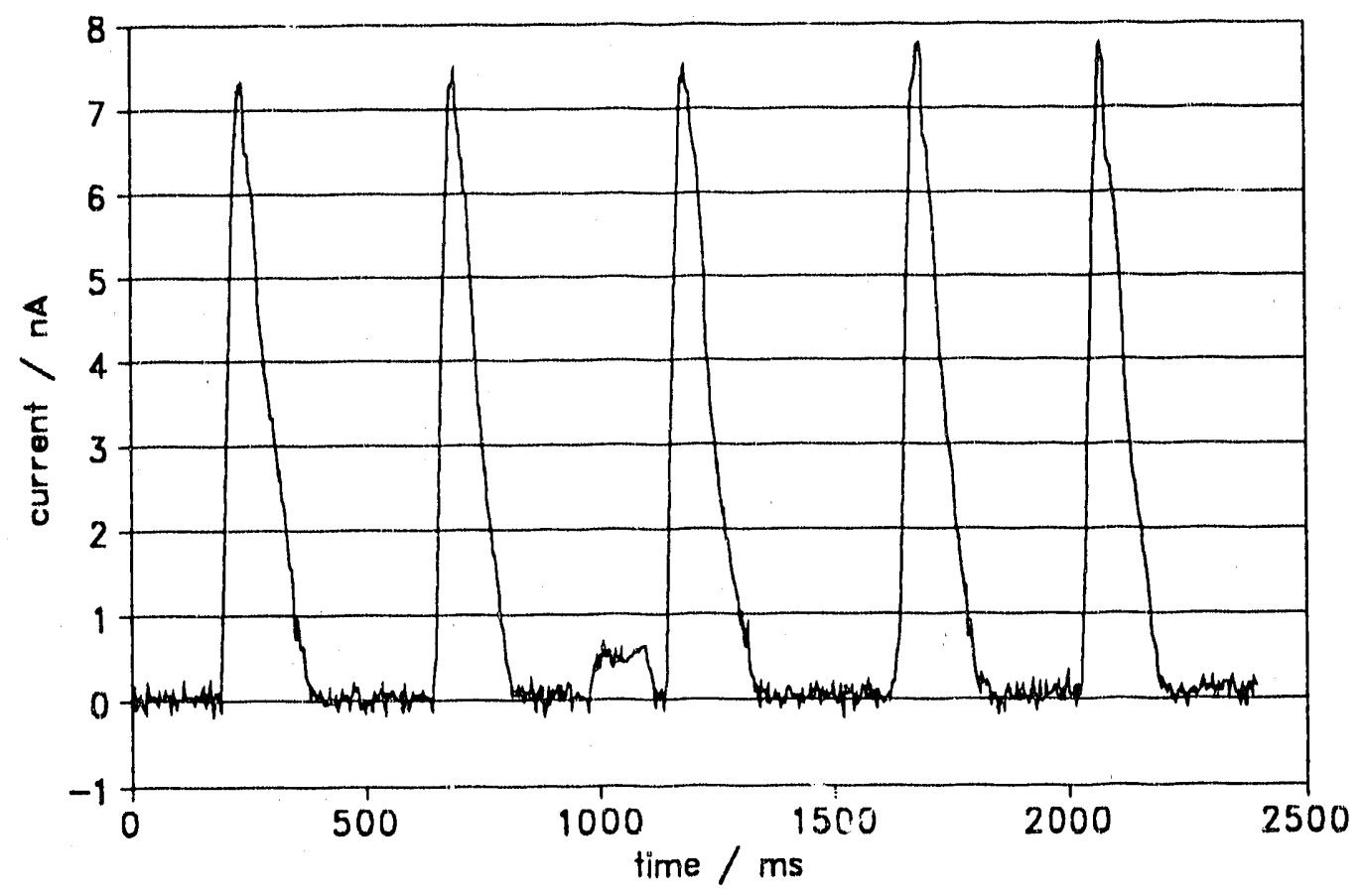

illustration 18 :

Subset of illustration 16 showing a regular series of five growth events. The small event appearing around $1000 \mathrm{milliseconds}$ into the plot is from activity on one of the other small facets exposed to the solution, see illustration 17 a). Growth conditions here are as in illustration 15. Each of these growth events occurs on a similar surface geometry with a microfacet size $h$. The waiting time discussed in the text is the time delay between the contact of a growing layer to the microfacet edge (peak of growth event) to the start of the next growth event. 


\section{Microfacet Size vs Square Root of}

Waiting Time

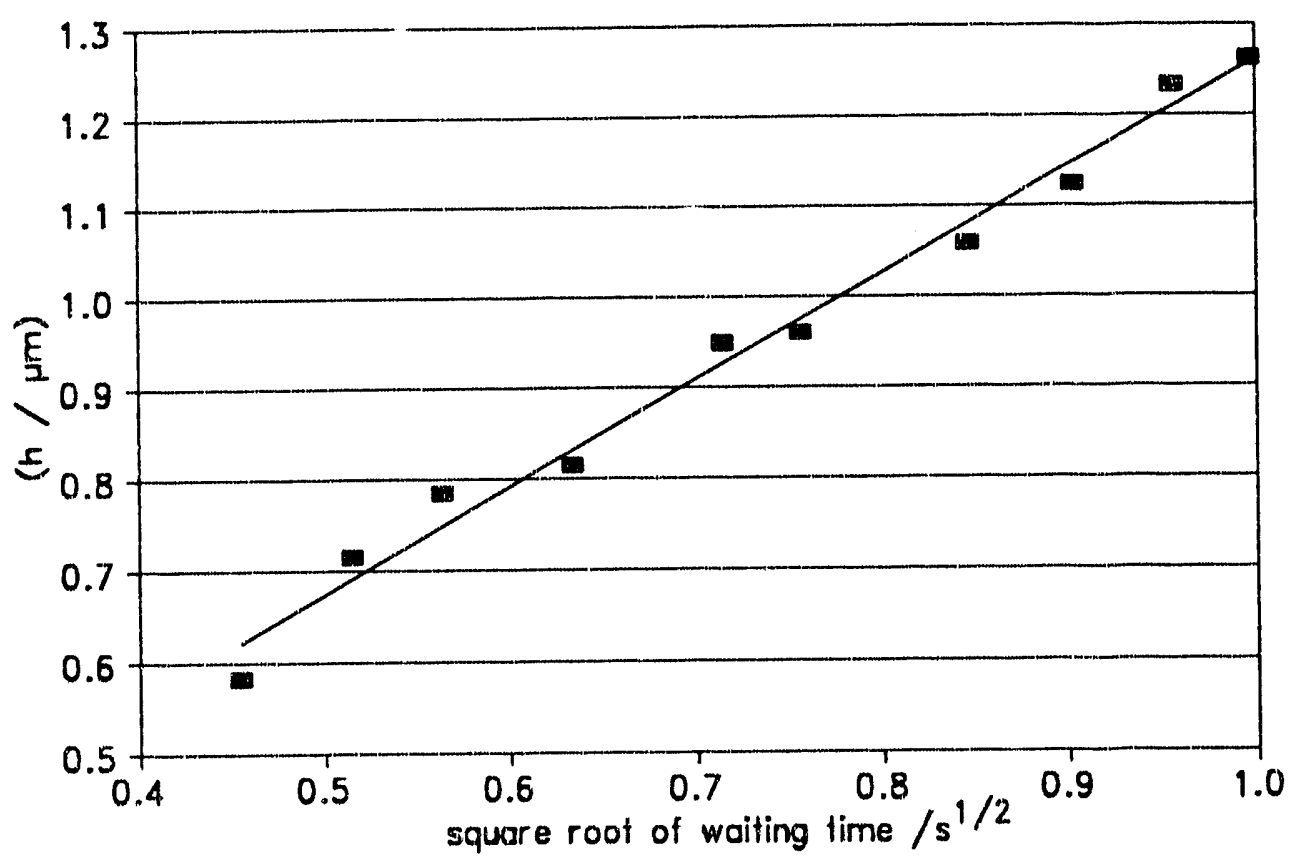

\section{illustration 19:}

sumary of deterministic growth observations from approximately 60 growth events at $T=20^{\circ} \mathrm{C}$. Individual series like illustration is are compiled together by averaging waiting time and microfacet aize of 5 to 10 growth events. The least squares fit line is shown; $r^{2}=0.998$. The non-zero vertical intercept provides an independent manure over the data set of the distance $\langle h-d\rangle$ as defined in illustration 9 . The 958 confidence range for this intercept contained the typical value used to nake the best-fit model curves as illustration 15. 


\section{CEAPTER 2}

COMPUTATIONAI MODELING OF GROWTA IN SIMPLE SYSTEMS

\section{Introduction:}

Geometric analysis of microscopic growth events has been used previcusly to locate initiating sites and determine kinetic parameters $26,27,29,44$. Previously published work has determined analytical and approximate descriptions of expected total growth rates given an initial configuration and other relevant parameters $7,45-50$. Although we focus here on measuring total growth rates at the interface through electrical current, there are other methods of monitoring surface and interfacial reactions. The discussions here and elsowhere can be extended to any technique that is capable of observing a total rate of reaction at the surface or interface. When the dimensionality of chemical reaction is restricted to that of the surface, geometric interpretations of the total rate of reaction can be very powerful tools. It is possible to derive the expected time-dependent total reaction rate for a given configrration. Additionally, inversion of the total reaction rate vB. time can be accomplished analytically in opecial cases to extract the geometric configuration. These derivations and inversions for the case of two-dimensional growth have been reatricted to very simple cases for algebraic reasona.

The most simple case of two-dimensional growth, the growth of a circular phase upon a circular substrate, has been treated by previous work. Analytical results for simple boundaries and single clusters 50 
have been derived as well as approximate results for multiple numbers of clusters ${ }^{4-49}$ and, recently, for multiple electrode arrays 7 . The observable quantity is a total reaction rate as a function of time. These model calculations are an effort to convert this observable quantity into parameters which describe issuss involved in microscopic growth. For the case of a single circular cluster, the expected total reaction rate as a function of time is easily derived for boundaries of a circle, equare, or other simple polygons. The time-dependent reaction rate depends on both the microscopic expaneion rate of the cluster and the initial location of the cluster. For these simple geometries, it is possible to invert the entire time series of the reaction rate for a growth event to extract an initial location. However, the analysis of time series of this type is usually performed through analysis of critical points in the time series. Analytical attempts have so far failed to determine the time-dependent total rate of reaction when the boundary is an ellipse. This is due to the complexity of the model when expressed as completely general two-dimenoional problem.

We have applied computational simulation to this problem and to the diffusion of species under arbitrary boundary conditions. For the growth of a circle into an ellipse, a number of techniques were applied with varying degrees of approximation. Along with simulations of the expected reaction rate, algorithms were developed to automate analysis of data. These algorithms used the simulated growth curves and extracted critical points for a number of simulated growth events. The set of critical points was then used to calculate the eccentricity of the original boundary and the initial locations of the growth events. A 
subset of the simulation of the growth of a circle into an ellipse is the modeling of the time series which we observed experimentally (see chapter 1).

simulations of random walks were run on a computer to study both single event arrival times and statistically averaged quantities. The random walk simulation probed cases where diffusion from an equilibrium region fills a population-depleted region. This chapter is divided into three sections to cover these subjects. The first section presents a discussion and simulations of a circular cluster growth into an elliptical boundary. The second section shows how the experimental time series were modeled. The last section presents our techniques and results in the random walk simulations.

\section{Section 1: circle into Elliose}

The ability to analyze a growth event at a surface or interface and extract kinetic and positional parameters is very important. The microscopic expansion rate of a cluster can be a tool for the investigation of the mechanism of the growth reaction. Very simple experiments, or at least conceptually simple experimenty, can be employed to determine how microscopic kinetics and mechaniem are affected by factors such as temperature and supersaturation. Positional parameters are useful in the study of growth events in many ways. For example, series of events can be studied for positional correlation. strong positional correlation between events can indicate that opecific sites at the surface or interface are promoting nucleation. Even the 
spatial propagation of the nucleation promoting site can be followed when a small number of sites are active. Another use of the nucleation position information is as a guide for subsequent high resolution surface probes. This potentially enables atomic resolution of a growth promoting site if the growth experiments can be conducted in the presence of an atomic force microscope 51 or scanning tunneling microscope52. Previous anelyses of growth of silver thin films have shown at least moderate agreement between experimental and geometric models, and have demonstrated potential for further study in location and structure of nucleation promoting sites.

The agreement of the experimental results and simple models has been large'y due to the experimental conditions. Under the growth conditions used in these solution phase experiments, the growth is under kinetic rather than thermodynamic control. Rinetic control causes the flat two-dimensional clusters expanding on the aurface to take a circular shape and grow with an isotropic expansion rate. As the clusters approach boundaries, they continue to behave like circles and the interaction between the new layer and the wall remains aimple. When the system under study follows these approximations, simple geometric models as discussed here and elsewhore are valid.

Positional analysis of growth events by geometric interpretation of total reaction rates is reatxicted by oymetry in the growth oystem. The total reaction rate of an isolated growing cluster can be expressed as a function of a microscopic rate multiplied by the total cluster peripheral length. Initial positions on the oubstrate which produce identical peripheral lengths as a function of time are imposaible to 
distinguish. Illustration 20 shows two examples, that of a circular cluster growing within a rectangle and within a circle. For a rectangle, all the positions where growth initiated are mapped onto one quadrat of the total surface. For a circle, the symetry of the system maktwa problem one-dimensional, producing a map of radial positions and no angular information. For positional analysis, mapping the data into a quadrant is obviously useful. Radial information has been used to check for signs of non-random nucleation 26 ; however, the power of this interpretation is limited. Single crystal planes within a circular capillary will produce an elliptical boundary if the surface normal does not point in the same direction as the capillary bore. The eccentricity of this ellipse breaks the circular symetry and allows mapping positional information to a quadrant of an ellipse. Therefore, for positional analyses of growth events occurring on substrates within circular capillaries, a little misorientation can be beneficial.

Previous analyses of growth events have applied special regions of growth events, critical points, to distill geometric information from the data 44 . The analysis process with this technique has two major steps. Several of the observed growth events are pulled at random from a data set of potentially hundreds or more events. The pulled growth events are studied for characteristic feutures. Useful regions are periods of linear growth, regions of disconkinuity (critical points), and overall curvature during the growth etwent. The characteriatic features are used to develop a self-consistent geometric nodel for the boundary and the groving phase. Once a reasonable geometric model is 
developed, it is possible to completely describe all the growth events in the data set by reducing the data to critical point information.

Data set reduction by critical point analysis is important in these eystems due to the number of points which are recorded in a growth event. With typical data acquisition rates, a single growth event occurring on a $5 \times 10^{-4} \mathrm{~cm}^{2}$ silver crystal surface can create a data curve with 400 to 40,000 raw data points. To obtain a thorough analysis of a system many (tens to thousands) of these growth transients should be recorded and analyzed. For systems where analytical inversions from reaction rates to geometric information exist, it would be very computationally intensive to handle every data point. The best argument for data set reduction via critical point analysis, however, is that it work very well. Once a geometric model is shown to be descriptive of a system, any observed growth event may be simulated with a small number of input conditions. The key to this analysis is the conversion of the critical points as extracted into initial position and kinetic parameters.

As an brief example, the simulation of a circular cluster growing into a circular boundary will be presented here. Illustration 21 shows a new circular layer in various stages of growth on a circular boundary and the resulting growth current signal. There are three critical points in the growth reaction. The first is the start of the growth event. The second occurs when the cluster makes its first contact with the boundary. The final critical point occurs when the substrate is completely covered. For this curve, two critical times, $t_{1}$ and $t_{2}$, are defined as the time from the first critical point to the second and 
third critical points respectively. The initial slope of the growth event is geometrically related to the microscopic expansion rate by (the total growth current gives reaction rate);

$$
\frac{d i}{d t} \propto 2 \pi v^{2}
$$

where $v$ is as defined in chapter 1. A combination of the initial slope and one of the critical times, $t_{1}$ or $t_{2}$, is sufficient to determine the position on the surface and completely define the simulated curve in the illustration for any type of units. To complete this example, illustration 22 shows three growth events resulting from three different initial positions.

For boundaries with a large number of edges, e.g. a square or irregular hexagon (both experimentally common) ${ }^{21}$, the number of critical encounters of the new layer with the boundary increases, increasing the number of critical points. Since the microscopic expansion rate is the velocity of the expanding layer across the surface, the critical times $t_{1}, t_{2}, t_{3}, \ldots, t_{n}$ can be related to distances. These correspond to the distance from the origin of the new layer to the surrounding edges of the boundary. In the case of a circular boundary, the second critical time is redundant; however, this is not the case when the boundary is rectangular or elliptical.

A useful feature of critical point analysis is the possibility for computerized automation. Although visual assessment is the most efficient method for correlating the observed growth events to a surface geometry, computer driven analysis can quickly process a long time 
series of data. an experimental time series of slow growth on silver single crystals consists of regions of zero current and current fluctuations resulting from nucleation and growth of two-dimensional layers. A critical point analysia program could skip through regions of zero current and find growth events by a level-crossing test. Once the growth event is found, least squares fitting of the early values generates the initial slope and the time position of the origin. sharp deviations or discontinuities can be correlated to the critical points. Finally, the tail of the transient can be fit to determine the final critical point.

The details of modeling the growth of a circle into an ellipse and the computer-based analysis of these simulations have been studied here. simulations were written and run in the ASYST scientific programing environment (Macmillan software). The simulations utilized both the array math and graphics capabilities of this programming environment. simulations were Iun on an AT\&T 6300 plus IBM compatible computer. The computer used an $80286 \mathrm{CPU}$ at $12 \mathrm{MHz}$ and an 80287 math coprocessing chip. Three basic algorithms were investigated in the modeling of growth in this system. Cxiteria for a succesaful model were the ability to generate accurate reaction rate vo. time curves and a short total run time. Oncr: a model was selected, several hundred growth events were calculated under different boundary conditions. The analysis techniques were developed on these data sets. The source code used for simulating growth events and the code for the analysis routines are included here in appandix $c$. 
The first simulation algorithm attempted to describe the surface as a square grid of points. Each site on the surface was assigned a non-zero value if it was populated by the new layer. The difference in the sum over the grid at $t_{i}$ and the sum over the grid at $t_{i+1}$ gave the "charge" passed in the time step. A circular or elliptical mask could be used over the grid to provide the appropriate boundary conditions. These simulations were hampered by speed and memory considerations. These restrictions fixed the grid sizes at 100 by 100 points or smaller. Typical simulation runs required five to fifteen minutes to complate for a single growth event. With this grid size, the interactions with the boundary produced a sawtooth shape on portions of the simulated reaction rate curves. This was caused by the inability of a coarse square grid to smoothly reproduce a circle. Real crystals within macroscopic boundaries do not show this effect because the atomic grid is three orders of magnitude smaller.

The second simulation algorithm used a shell of points at a radius x. The shell points were checked at each time step to verify the number within tho boundary. Between each time step, I was increased. The points within the shell were angularly positioned at random before the start of the growth event. The reaction rate was a function of the radius I multiplied by the number of shell points within the boundary. Typically, 100 shell points were used and the results of ten runs were averaged together to produce a simulated growth event. A single run required three minutes. The ten run average was effective in removing sampling errors; however, a simulated growth event required thirty minutes of computer time. 
Converting the shell technique from a set of randomly positioned points to an equally angularly spaced grid eliminated the need for averaging of the runs. With 360 points with $1^{\circ}$ spacing, a growth event oimulation required five to eight minutes. The radial resolution in these simulations produced data which was one tenth of tho expected experimental resolution. This resolution was estimated by the precision of our current measurement and the temporal spacing between experimental data points. Each grid point in the simulations corresponds to a surface area containing $10^{5}$ atoms. A pair of simulated growth events created by this algorithm is shown in illustration 23 . These two simulations show the difference in growth event shape when the initial clugter position is along the major and minor axes of an elliptical boundary. Typically, growth event initial positions were chosen at random by the computer. Series of transients for a set surface boundary were calculated in blocks of 100 and saved as binary files.

In order for a computer to perform critical point conversion to locations, the boundary must be determined. For an elliptical boundary, the length of one axis and the eccentricity are sufficient to describe the boundary. one of these factors, the minor axis length, is always known. Wuen the surface normal is aligned along the capillary, the boundary is a circle with a diameter equal to that of the capillary. As the surface normal rotates off the direction of the capillary bore, the intersection of the plane of the surface with the capillary gives an ellipse, and the minor axis is the capillary diameter. To determine the eccentricity of this elipse, the relative length difference between the 
major and minor axis can be used. This is obtained from the experimental data by constructing what we call a rainbow plot.

These rainbow plots are constructed from the sums of the critical times extracted from the growth events. Illustration 24 shows a rainbow plot for a set of 100 growth events which originated rit random surface positions. The spread in growth times is a result of the different distances over which the new layer expands. The number of events which occur for a particular $t_{1}+t_{2}$ takes on the shape of an arc for a theoretically perfect distribution. This "total" growth time is a crude measure of the angular position. The events which take the longest to complete started at small angular deviations from the major axis. The events which have the shortest time to completion began near the minor axis. Along these two axes, multiplying the expansion velocity by the time $t_{1}$ gives the distance from the nucleation site to the nearest wall, and multiplying the expansion velocity by the time $t_{2}$ gives the distance to the farthest wall. By adding these two times together, a value which is proportional to the distance of the axis is obtained. This is true for all radial positions. The endpoints of the rainbow plots determine the relative difference between the major and minor axes lengths.

There were three steps then, in the conversion of a simulated or experimental data set into an original position. First, a computer routine scanned over a large number of growth events, extracting the critical times. Second, these critical times were used to determine the relative difference in length between the major and minor axes, providing an approximate boundary shape. Third, a computer algorithm 
used the critical times for a particular growth event and the determined boundary to calculate the nucleation site for that growth event.

The computer analysis program used to analyze the simulations did not have to construct Iinear fits to the start of the events because the starting point of each event was the first point in the run. The time $t_{1}$ relative to the starting point was determined by looking for the first deviation from linear behavior. Once this first point had been determined, the computer scanned the data set until the simulation data dropped below a pre-set value. The previous five data points were fit by least-squares linear regression. From the regression coefficients, the intarcept signaling the end of the growth event was determined. This gave the critical time $t_{2}$.

The special significance of the critical times as discussed earlier is that they define the radius of the expanding layer when important interactions with the boundary occur. The first critical time, $t_{1}$, is the time of first interaction of the cluster with the boundary. The cluster radius at this time, $I\left(t_{1}\right)=v t_{1}$, is the distance from the cluater origin to the nearest point on the boundary. Given $t_{1}$ and a boundary shape, a set of possible nucleation sites can be constructed; this is shown in illustration 25. At the second critical time, $t_{2}$, the growing layer has reached the point on the boundary most distant from the nucleation site. The computer analysis algorithm used this second time to select the origin from the possible sites ohown in ilustration 25 .

The position determination algorithm modeled the set of possible nucleation sites as shown in illustration 25 as an ellipse. The 
eccentricity of the ellipse was determined by the possible sites along the major and minor axes of the original boundary. The second critical. time was converted into a distance. The computer then searched for the interior normal to the boundary with this distance to the ellipse of possible nucleation sites. The normal to the ellipse approximates the farthest point from the nucleation site and is exactly correct along the major and minor axes. Previous algorithms for circle-into-circle position extraction used a line that passed through the center of the circle to describe the length $v \cdot\left(t_{1}+t_{2}\right)$. This is rigorously correct because of the radial symmetry. For an elliptical boundary, this approximation was tried and it provided significantly poorer agreement when comparing the initial and calculated nucleation sites.

From our analysis of the simulated growth as described above, we concluded that the extraction of initial positions from experimental data is feasible. A small number, 50 to 100 , of simulated grovith events randomly distributed on the surface were found to allow accurate determination of the eccentricity of the boundary. This came as a surprise since it was expected that a large number of growth events would be required to sample the surface. The rainbow plots presented a distribution close to the theoretically expected distribution with a small number of runs. A set of 300 simulated events gave agreement to the known eccentricity within 18. Since previous experimental work has been successful in capturing several hundred growth events over a shoxt period, experimental deternination of the surface boundary should be possible. 
Converting the critical times extracted by the computer into coordinates by the algorithun developed was time efficient and accurate. Illustrations 26 and 27 show quadrants of a simulated surfiace at two accentricitiea. The initial and calculated nucloation sites are shown for 25 simulated runa. The radial agreement of the calculated position and the initial positions is very good. The angular agreement is not as good near the major and minor axes. Some of the deviations are due to the approximationg in the position extraction algorithm. The ansumption that the net of posaible initial locations can be described as a mecond ellipre fails for long $t_{l}$. This approximation oeems to have the atrongest impact on determining positions near the major and minor axes. Near the boundary, the angular coordinates are affected by the precision used in determining the intersection of the surface normal with the set of possible positions.

Bigher precision in the calculations is possible for computer generated data seta. Bowever, the precision used in determining the positions shown in illustrations 26 and 27 are consistent with presently available experimental revolutions. From these simulations of growth events we have drawn several conclusions.

Simple computer modelo can successfully simulate growth evento for arbitrary and unusual boundaries. Models were able to determine curves with close to exparisantal resolution in short periods of time. This allows user-driven interactive fitting of data if required. The application of acientific computing workstation would allow interactive simulation of data at the experimental resolutione. Computer based analysis of these events was succesaful due to the 
distinetive nature of the critical points within the events. In the case atudied here, numerical conversion from critical times to surface coordinates is fairly ouccessful. We find that radial positioning is well-determined for the growth of circles into ellipses. The angular resolution is not as good, yet still good enough to reveal the approximate positions. Poaitional location within such a boundary opens the posaibility for microscopic study of Bites which are responsible for nucleation at the surface.

Yocieling Experimental Datai

The shape of the growth event and the total charge passed in the growth events described in chapter 1 implied that a subset of the area within the copillary was the active ourface. Several potential surface geometries and cluster shapes were explored in a manner oimilar to that of the previous section in order to find a sensible geometric model to describe the data. The model which was most consistent with the experimental data is shown in illustration 9 and reproduced with appropriate labelo for this section in illustration $2 \mathrm{Ba}$. The surface region which is active is a sector of a circle or ellipse. A computer. prograrn written to plot the data was modified to include a model calculation of the growth of a circular cluster on this active aurface. The adjustable parameters in the model plot were selected by the uBer. The modeling program constrained the location of the initial cluster to the region at the capillary wall. The critical times used to generate the model were input by the user through picking points on the 
experimental curves. The eccentricity of the region and the shift along the capillary towards the facet edge ( $h$ - d) were selected by the user and adjusted for the best fit with the experimental data.

Illustration $28 \mathrm{a}-\mathrm{d}$ shows the basis for the model calculations and shows the various geometric regimes within the model. Since the geometry was restrictive, it was feasible to derive simple expressions for the current as a function of time. The form of the expresaion varied with the growth regime. The model program uses three regimes and calculates (numerically) the intersection point between the boundary and the growing layer. The growth current under this model is proportional to the radius of the cluster and the angular separation of the intersection points.

The first regime is shown in illustration $28 \mathrm{~b}$. Here, the cluster is unbounded on one side and is in contact with the edge of the crystal at the capillary wall. Thi period of the growth cycle in identical to the initial growth period shown in illustration 22 (curve c). Using the intersection points shown in illustration $28 \mathrm{~b}$, the current is given by:

$$
i \propto r \cos ^{-1}\left[1-\frac{\left(x-x^{\prime}\right)^{2}+\left(y-y^{\prime}\right)^{2}}{2 r^{2}}\right]
$$

The intersection points $x, y$ and $x^{\prime}, y^{\prime}$ are determined numerically for a particular I by finding the value:

$$
x_{e}=\sqrt{A^{2}\left(1-\left(\frac{y}{B}\right)^{2}\right)}
$$

within $\varepsilon$ of the value: 


$$
x_{c}=\frac{\left(\frac{I}{B}\right)^{2}-\left(\frac{I}{A}\right)^{2}+2\left(x_{0}-Y_{0}\right)+2 y\left(1-\left(\frac{I}{B}\right)^{2}\right)}{2\left(1-\left(\frac{Y}{B}\right)^{2}\right)}
$$

(14).

The deviation value typically used was $\varepsilon=0.001 \mu \mathrm{m}$; in the two expressions above, $x_{\theta}$ is from the shape of the elliptical bourdary and $x_{c}$ results from the intersection of a circle of radius $r$ located at $x_{0}, y_{0}$ with that boundary. As $x$ increases, the two solutions to these equations, $x, y$ and $x^{\prime}, y^{\prime}$, move away from the growth origin.

The second regime is shown in illustration $28 \mathrm{c}$. In this period of the growth cycle, there are four intersection points of the critical cluster with a boundary: $x, y ; x^{\prime}, y^{\prime} ; x^{\prime \prime}, y^{\prime \prime}$; and $x^{\prime \prime \prime}, y^{\prime \prime \prime}$. The firat two are given by equations 13 and 14 , as in the first regime. The other two intersection pointe result from interaction with the facet edge and they are given by:

$$
\begin{aligned}
& x^{\prime \prime}=x^{\prime \prime \prime}=h-h \\
& y^{\prime \prime}=y_{0}+\sqrt{r^{2}-\left(x^{\prime \prime}-x_{0}\right)^{2}} \\
& y^{\prime \prime \prime}=y_{0}-\sqrt{r^{2}-\left(x^{\prime \prime \prime}-x_{0}\right)^{2}}
\end{aligned}
$$

The current is given by:

$$
i \propto r\left[\cos ^{-1}\left[1-\frac{\left(x-x^{\prime}\right)^{2}+\left(y-y^{\prime}\right)^{2}}{2 r^{2}}\right]-2 \cos ^{-1}\left(\frac{x^{\prime \prime}-x_{0}}{r}\right)\right]
$$


The third regime is shown in illustration $28 d$. At this point, the new layer has expanded over most of the surface and has completely stopped growing on one side. The current resulting from filling the remaining portion of the substrate is given by:

$$
i \propto r \cos ^{-1}\left[1-\frac{\left(x^{\prime}-x^{\prime \prime \prime}\right)^{2}+\left(y^{\prime}-y^{\prime \prime \prime}\right)^{2}}{2 r^{2}}\right)
$$

where $x^{\prime}, y^{\prime}$ and $x^{\prime \prime \prime}, y^{\prime \prime \prime}$ are as defined above; this result is analogous to equation 12 .

The modeling program calculated the expected current for a hypothetical clustex as a time series otarting at the user input origin. The program increased the size of the cluster in each experimental time step by the distance indicated by the initial slope of the experimental data. The radius of the cluster at each time step was compared to the gennetry to determine the relevant growth regime. The appropriate equation above was then used to determine the model instantaneous current. For the simulations of growth events occurring on these approximately $10 \mathrm{\mu n}$ wide crystal facets, the total time to generate 300 model points was approximately 4 minutes.

The model for the surface and growing cluster used in this section was selected on the basis of its match to known geometric constraints and its flexibility. Some example output from this modeling routine is provided in illustrations 29 and 30 . The variation in basic form as the eccentricity increasea is shown in illustration 29. These model curvos are dispiayed in arbitrary time units to emphasize the changes in the 
position of the peak of the transient with respect to the start and end time. As the eccentricity increases, the first regine growth signal deviates from linear behavior. Comparing models of growth events of equivalent charge, it was found that the first critical time, the time to the peak of the event, increased with increasing eccentricity. A change in eccentricity is indicative of change in the tilt of the active surface. For all series which we observed, the eccentricities were gelf-consistent from growth event. to growth event. Bowever, analysis of individual series corresponding to different active surfaces revealed a range of experimental eccentricities. This it best explained by the presence of multiple crystal facets, with different angular orientations (c.f. illustration 17a).

Illustration 30 shows the effect of moving the nucleation site along the edge of the capillary. For simplicity in the model, the ahift in position is represented as distance shifts with respect to the microfacet size h. Angular position is determined by solving for position using the known boundary geometry. shifts in the initial position of the cluster toward the facet edge cause longer total growth times and make the extent of the final growth regime longer.

Illustration 15 from chapter 1 shows on experimental growth event and a model curve. The total charge under the model curve is equal to the growth event charge. The model curve was generated at an eccentricity of 0 , meaning that this active surface had a normal close to the direction of the capillary bore. By adjusting the position of the initial cluster, the final portion of the growth event is properly fit. Typically, a range of distance shifts $(h-d)$ produced equally 
good fits. The range of values was around 308 of the total distance shift. Although the experiment and model do not show an exact point-topoint correlation, the experimental and model curves show the same number of growth regimes, in approximately the same places. The quality of the fits between the experiments and model is better in this work than in work reported elsewhere $26,27,29$, this is due to the flexibility and appropriateness of the geometric model.

The range in distance shifts of the initial cluster generates two rather wide portions of possible nucleation site locations. Illustration 31 shows this shortcoming. For the set of events shown in illustration 18 , the average shift is $630 \mathrm{~mm}$ and the range is $\pm 100 \mathrm{~mm}$. This creates a region along the boundary about $1 \mu \mathrm{m}$ in length of possible positions. The actual active site is probably not that large, our data quality simply prevents interpretation with better resolution. Based on the deterministic rature of the observations, we have been able to conclude that a single portion of the surface was active; however, we are not able to count the number of sites within this region.

The quality of the fit obtained to the experimental data by a model which treats the new layer as a circular entity is intriguing. Under these conditions, the new layer seems to be growing under kinetic control. Thermodynamic control. would produce small polygons on the surface. The polygons should provide significantly different eritical behavior than what is observed. Within our experimental resolution, kinetically limited growth with a single isotropic expansion rate is the best explanation of the observed growth behavior. Near the maximum current in the data, there is a deviation from the model curve. This 
deviation may be signalling the fajlure of the circular cluster model or may reflect a deviation from a straight line at the facet boundary. This is the major shortcoming of this modeling technique; in the end, there are basic assumptions which must go into the model before interpretations can be made. However, the strength of the growth and surface event analyses presented in the previous two sections is that they can quickly provide information about active Burface sites and kinetic parameters. Future work may be able to use these analyoir techniques as the starting point for probes which have finer resolution but are unsuitable for application to realistic surfaces because of their size.

Modeling surface Diffusion:

The surface motion of absorbed species plays an important role in many aspects of chemistry and physics at surfaces and interfaces. The behavior of our observed regular growth events as discussed in chapter 1 appears to be another eximple. Species transport by diffusion is a topic which has been well developed. Previous analytical and computational treatments of surface self-diffusion have invertigated concentration effects and adsorbate interactions53-55. The most common description of atomic or molecular diffusion is based on a random walk. In this random walk, the particle's trajectory is determined by motion f. Jm site-to-site. The probability of hopping from one site to another is independent of the direction of the hop and no memory of the previous hop direction is maintained. Solution of Fick's Second Law under the 
appropriate boundary conditions for a free standing particle(s) leads to:

$$
\left\langle I^{2}\right\rangle=2 \mathrm{dDt}
$$

where d is the dimensionality of the system, $\left\langle r^{2}\right\rangle$ is the mean mquared displacement of the particle(s) at time $t$ and $D$ is the diffusion coefficient. The mean squarad displacement is used as a descriptive quantity because the hop statistics constrain the mean displacement to zero.

This functional form is consistent with our obrervations; however, it does not provide the correct conversion from the slope of the size vo. square-root of waiting time fits to the diffusion coefficient. In the experiment, the boundary conditions are consistent with diffusion from an area of oquilibrium concentration across a depleted region. The capillary wall produces a boundary which prevents motion in one directiol, beyond a certain point. The experiment does make a clear and simple measurement of the transport between these two boundaries. Bowever, since one boundary is alightly elliptical, derivation of a simple two-dimensional analytical result proved quite difficult. The regular growth events provide the data from pseudo-onedimensional diffusion experiments. The equilibrium region acts as source. The nucleation site at the capillary wall creates a growing cluster which is detected by the steady-state current measurement. The time from a growing layer's contact with the facet edge to the atart of 
the next event determines the time required for surface species to diffuse from the source to the nucleation site.

The waiting time between growth events of similar total charge (hence, similar facet eize) varied slightly. Variations in the waiting time can be caured by the nucleation process or by a epread in surface species arrival times. To determine the magnitude of these variations and their implications for the nucleation process, groups of events of the same facet size were compared. The waiting time was divided into two parts; the minimum time required for surface apecios arrival, and a variable eluster arrival time. The typical variable arrival time was about 108 of the minimum waiting time. As stated earlier, the cluster arrival time technically reflects both the time variance of the nucleation process and surface diffusion. The variable cluster arrival time can be used to place an upper bound on the critical cluster oize in the nucleation process. The variance of the variable cluster arrival time is equal to the nucleation rate, under the assumption that the nucleation process can be described as a Poisson process.

The upper bound to the nucleation rate indicated that very omall critical clusters were involved in the nucleation process. In fact, an atomistic treatment of the nucleation process might be more appropriate to determine the actual critical size because the bajance of energies nucleation equation predicts a critical cluster with less than 5 atoms. Regardless, the ypeer bound in critical cluster size is very small, implying that the diffusion process need only provide a few surface species before the cxitical cluster forins and growth begins. We have tried computational simulation as an approach for studying the effecto 
of random surface apecies motion with respect to this problam. The simulations studied the arrival times of hypothetical diffusing species moving in a random walk. By conducting actual simulations, it was possible to select the arrival times for the first, second, third, etc. surface species. In the simulations, the hopping probability and the diffusion length were varied in order to study the spread in arrival times resulting from the diffusion process.

For surface diffusion of a small numbec or even a single species to be a viable explanation of the experimental observations, it needed to be shown that a random walk model produced a low epread in arrival times under the appropriate conditions. Our experimental results which measured the surfice transport of a species demonstrated only a 108 variation in the arrival time. In the limit of a mall number of hopping epecies diffusing over a short region, it is intuitive that the arrival time could fluctuate significantly. By modeling large systems, the efects on the arrival time statistics could be studied when the number of hop attempts moved into the area of $10^{6}$ to $10^{7}$.

The diffusional process was modeled by a random walk by applying a computer Monte Carlo method to each surface species. This method was chosen due to its ability to treat very large systems quickly. The total probability of morement in a time step was varied from 0.8 to 0.002 . The lower value represents the typical hop success based on the activation barrier to diffusion for fcc(111) silver as determined by molecular dynamics calculations 39,40

Both two-dimensional and one-dimensional simulations of the Burface were conducted. The resulto from the two-dimensional study will 
not be reported here. This is because the small number of surface sites required to make these calculations temporally accessible produced arrival times with a very large spread. The one-dimensional simulations provide a reasonable approximation to the problem since the diffusion region is a short line across the wide strip of the active facet, see illustration 32 .

A computer program was written in FoRTRAN to run sets of simulations. The simulations were run on IBM compatible parsonal computers with 80286 and 80386 central processors. Typical simulation times ranged from a few minutes up to a few hours. The program could be adjusted to allow verification of the simulation technique by equation 20. The simulation code is quite short and is included here:

C THIS IS TEE SOURCE CODE FOR TORSD5.FOR, A COMPLETE AND

C WORKING SIMUIATION PROGRAM. MICROSOFT FORTRAN 4.1

C TIM ROBINSON LAST REV $12 / 15 / 89$

BLOCK DATA DIFFUSION

COMMON /INFO/ ISURF(5000), BEOP, FBOP, IFAP, IEQ, ISEQ INTEGER* 1 ISURF INTEGER IFAP, IEQ, ISEQ

REAL BHOP, FHOP

DATA IFAP $/ 1 /$, IEQ $/ 3 /$, BHOP $10.200 /$, FHOP $/ 0.100 /$

DATA ISURF /5000*0/, ISEQ /40/

END

C BERE'S AN EFFECTIVE PORTABLE RANDOM NUMBER GENERATOR:

C FROM "NUMERICAL RECIPES", W. A. PRESS ET.AL., CAMBRIDGE

C UNIVERSITY PRESS, 1986, P. 195. NEGATIVE IDUM RESETS (See Ref. 56)

REAL FUNCTION RAN1 (IDUM)

PARAMETER ( $M=714025, I A=1366, I C=150889, \quad R M=1 . \quad / M)$

INTEGER * 4 IRADUM, IDUM

IF (IDUM .GT. O) IRADUM = IDUM

IRADUM $=$ MOD (IRADUM*IA+IC, $M$ )

RAN I = IRADUM $*$ RM

END

C INITIAIIZATION SUBROUTINE SUBROUTINE INITIAL 
COMMON /INFO/ ISURF (5000), BEOR, FBOP, IFAP, IEQ, ISEQ INTEGER*I ISURF

WRITE(*,*) 'INPUT HOPPERS, EQ. HOX SIZE, ROP PROB.'

READ (*, I) IEQ, ISEQ, PRYOP

QHOP $=$ PRROP

THOP = BEOP / 2 .

END

C SIMULATION ARRAX INITIAIIZATION SUBROUTINE

SUBROUTINE SETUP

COMMON /INYO/ YSURF(5000), BHOP, FEOP, IFAP, IEQ, ISEQ IWTEGER* 1 ISURF

DATA ID $/-1 /$

DO $10 I=1, I E Q$

$L=\operatorname{INT}(\operatorname{RAN} I(T D) * I S E Q)+1$

IFAP $M A N(L, I F A P)$

$10 \operatorname{ISURH}(L)=1$

END

C BORFING SUBROUTINE FOR FIRSTBOX

SUBROUTINE TIRSTEOP

COMMON /INFO/ ISUR (5000), BEOP, FHOP, IFAP, IEQ, ISEO INTEGER* 1 ISURE

DATA ID $/-1 /$

IMET = ISURY (1)

DO $10 I m 1, I M E T$

IF (RAN 1 (ID) .IT. FEOP) TEEN

ISURF (1)=ISURF (1) -1

$\operatorname{ISURP}(2)=\operatorname{ISURP}(2)+1$

END IF

10 CONTINUE

END

C EQUILIBRIUM SUBROUIINE

SUBROUTINE EQUIIIBRIUM

COMMON /INFO/ ISURF(5000), BEOP, FEOP, IFAP, IEQ, ISEQ INTEGER* 1 ISURF

DATA ID $\mid-1 /$

C COUNT BOPPERS IN EQUILIBRIUM REGION

ISUM $=0$

DO $10 I=1, I S E Q$

$10 \quad$ ISUM $=$ ISUH $+\operatorname{IEURF}(I)$

IDIFF " IEQ - ISUM

C ADD OR SUBTRACIS BOPPERS TO EEAD TOWARDS TEE EQUILIBRIUM VAIUTE IF (IDIFY. $\mathrm{EQ}$. O) THEN

IF (ISURFI(1) .GM. O) CAIL EIRSTHOP

ELSEIF (IDIFF .EQ. I) TEEN 


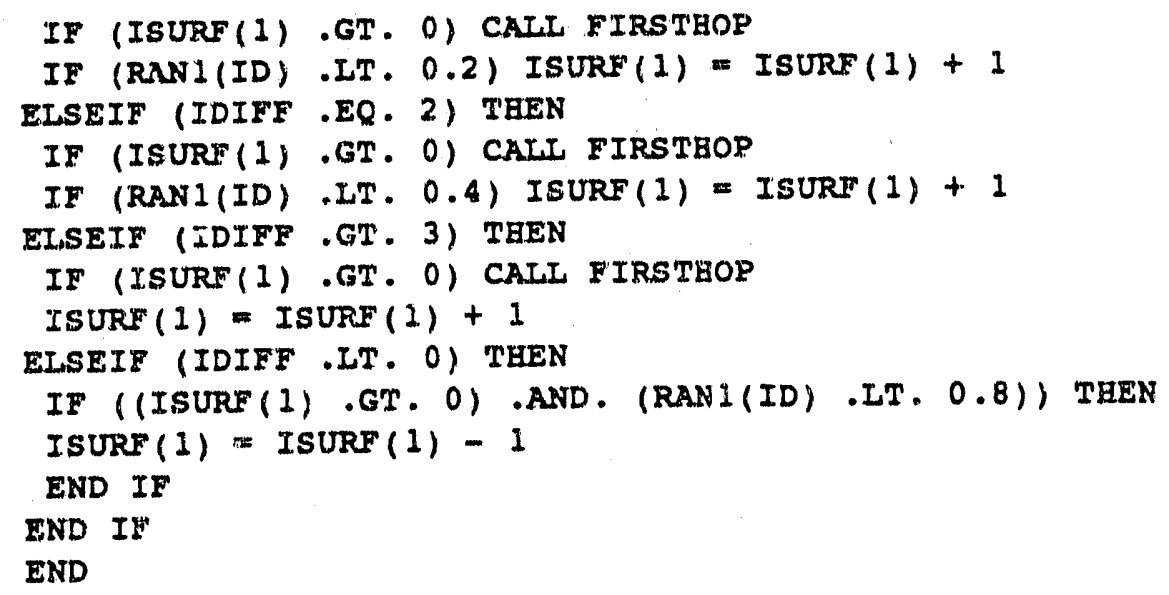

C MOVEMEN' SUBROUTINE

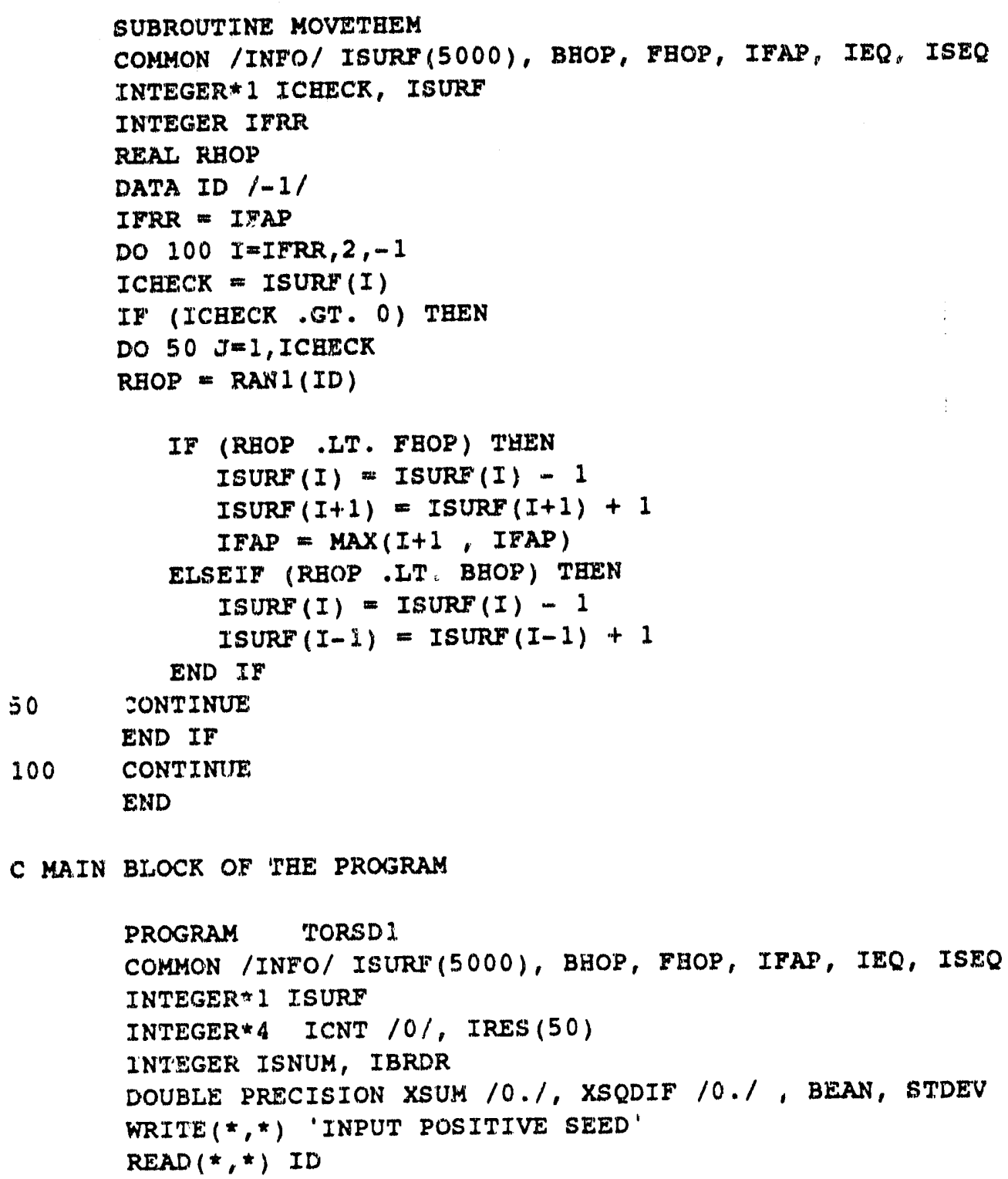

C MAIN BLOCR OF ITEE PROGRAM

PROGRAM TORSDI

COMMON /INFO/ ISURF(5000), BHOP, FHOP, IFAP, IEQ, ISEQ INTEGER* 1 ISURF

INTEGER*4 ICNT $10 \%$ IRES (50)

INTEGEK ISNUM, IBRDR

DOUBLE PRECISION XSUM $10 . /$, XSQDIF $/ 0 . /$, BEAN, STDEV WRITE $(*, *)$ 'INPUT POSITIVE SEED'

READ (*,*) ID 
$X=R A N \perp($ ID)

WRITE $(*, *)$ 'INPUT " 9 " TO CAANGE DEFAULTS'

$\operatorname{READ}(*, *)$ IANS

IF (IANS .EQ. 9) CAIL INITIAL

WRITE $(*, *)$ 'NUMBER OF SIMULATIONS (<*50)'

$\operatorname{READ}(*, *)$ ISNUM

WRITE $(*, *)$ 'LOCATION OF BORDER'

$\operatorname{READ}(*, *)$ IBRDR

IBRDR = IBRDR + ISEQ

C MAIN SIMUIATION BLOCK

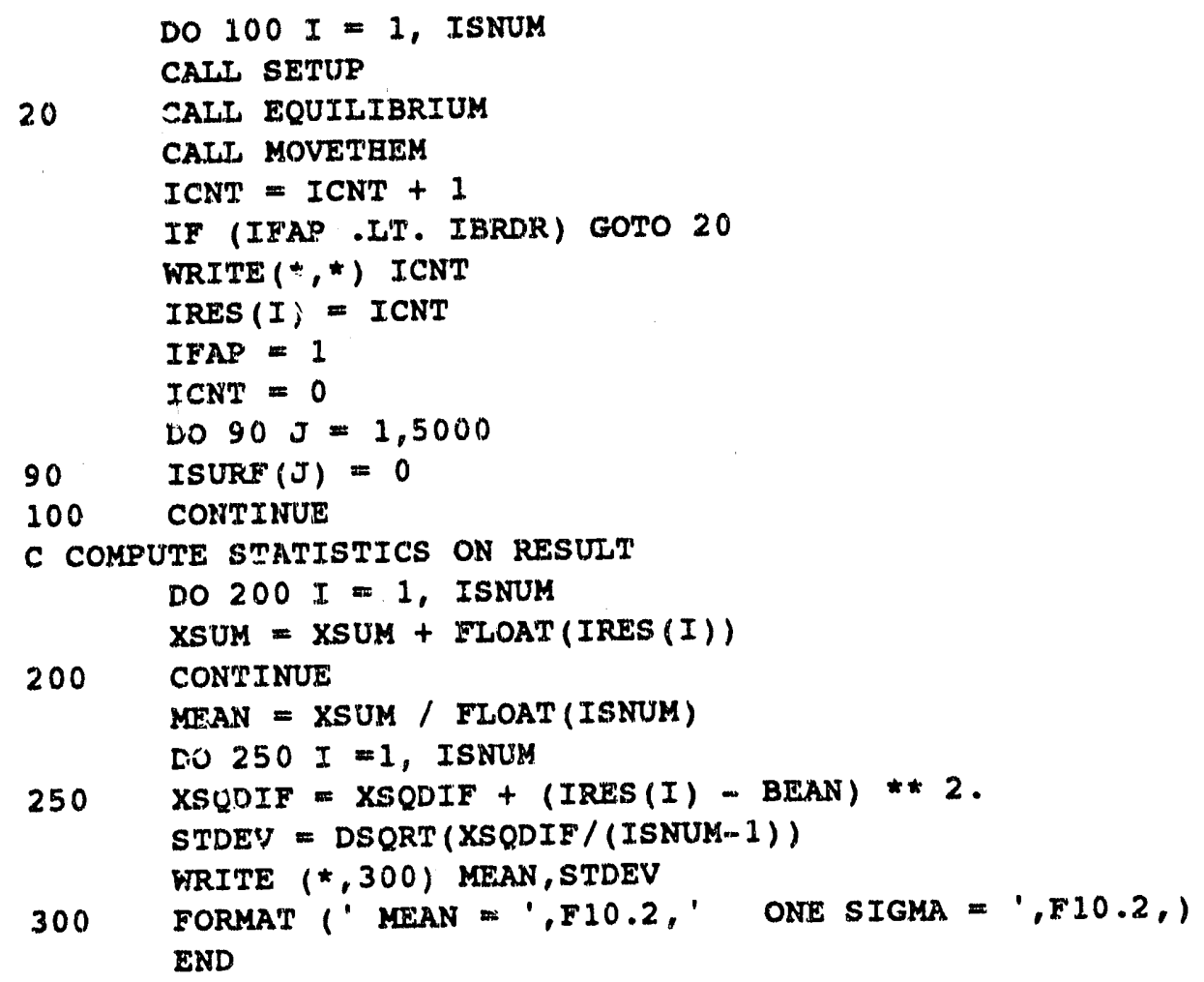

The one-dimensional surface region is modeled as an array of byte values (array ISURF), see illustration 32. The value contained in each array element is the number of surface species at that site. An adjustable size equilibrium region is located at one end. One subroutine in the program checks the population within this region at the start of each time step. The population is adjusted by introducing more surface species or removing them at the edge of the equilibrium 
box. All surface species were given equal probability of hopping to the left or right as in illustration 32 .

The simulation code stops the run when the farthest raach of one of the atoms stxikes the border region. Until then, the movement routine starts at the outlying region and works toward the equilibrium region, conducting hop attempts for all species at a surface aite. In developing simulation code, the effects of three sampling methods ware studied. The first simulation programs allowed a maximum of only one hop per surface species per time step. The overhead required to account for each surface species, individually, significantly slowed execution. The other two algorithms either scanned from the equilibrium region, making hop attempts for all species at a site, or scanned towards the equilibrium region as the code here does.

The problem encountered with the latter techniques was the occasional observation of double hops in a time step. At low hop probabilities, the chance of a double hop was reduced. At total hop probatilities of 0.2 or less, these latter two algorithms with the same input seeds produced arrival times within 108 of each other. After the majority of the simulations were completed, an additional algorithm was considered. It retains most of the processing speed of the code shown here and eliminates the sampling errors.

Instead of maintaining one array of values holding the contents of the surface sites, two arrays are used. On all the odd numbered time steps, the first array is used as the number of surface species at a site. The species after successful or unsuccessful hop attempts are placed in the appropriate location in the second array. On the even 
numbered time steps, the second array is used as the source for site contents and the results of the hop attempts get placed back in the first array. Between the time steps, the target array is cleared so that simple suming at sites produces the correct number of species at each site. This approach would lend itself well to massively parallel computing systems. In the near future, it should be reasonable to simulate this type of problem with atomic resolution.

our simulations used a range of diffusion lengths (b). Site numbers ranged from 50 to 1,000 from starting point to detection sita. The actual number of surface sites over a $10 \mu \mathrm{m}$ strip of a silver closepacked plane is around 50,000 . The results of simulation runs for the various diffusion lengths were expressed as a ratio;

$$
\beta=\frac{D}{D_{o b s}}
$$

where $D_{o b s}$ is the diffusion coefficient observed from the simulation and $D$ is the diffusion coefficient expected in the lack of a concentration gradient. The resulting $\beta$ values were plot.ted as a function of $1 /$. These plots showed a linear behavior over the values gtudied. Extending the results to zero $1 / /$ provides a nunerical estimate of $\beta$ for the experimental data.

simulations revealed that $\beta$ was a function of hopping probability, concentration within the equilibrium region and, as mentioned above, the diffusion length. These conclusions are consistent with accepted models of diffusion under similar conditions. A set of simulations for a given $l$ at various hopping probability were extended linearly to get the $\beta$ 
value used in the $1 / /$ plots. Table 4 summarizes values for $\beta$ for a range of hopping ptobabilities and diffusion lengths, using the equilibrium concentration of surface species as deternined by a.c. impedance spectroscopy in chapter 1. Illustration 33 shows the results extended to zero hopping probability and plotted v8. $1 /$.

\begin{tabular}{l|l|l|l|l|l|l|}
\multicolumn{5}{l|}{} & \multicolumn{5}{|l}{$\beta$ at Bopping Probability: } & Low Pr. \\
\hline length & 0.4 & 0.3 & 0.2 & 0.1 & 0.05 & limit \\
\hline 50 & 0.851 & 0.912 & 1.002 & 1.022 & & 1.098 \\
\hline 75 & 0.626 & 0.668 & 0.774 & 0.834 & 0.856 & 0.897 \\
\hline 100 & 0.496 & 0.555 & 0.593 & 0.633 & & 0.681 \\
\hline 200 & 0.330 & 0.368 & 0.411 & 0.380 & & 0.412 \\
\hline 500 & 0.225 & & & .250 & & \\
\hline 1000 & & & 0.218 & & & \\
\hline
\end{tabular}

Table 4. Simulation results for several diffusion region lengths and hopping probabilities. The right-most column contains the extrapolations to the low hopping probability limit used in illustration 33 .

The spread i.. arrival times for the first surface species fell with decreasing hop probability and with increasing diffusion length. For a hop probability of 0.2 and diffusion lengths of 50 , the standard deviation in arrival times for 30 runs was on the order of the average arrival time. For a diffusion length of 200 hops the standard deviation was half of the mean arrival time. The normalized deviation decreased linearly with the square-root of the diffusion length. This projects a standard deviation of 38 in the arrival times for the number of hops required in the experiment. The difference in arrival time between the 
first and the first few ourface species became very small when linearly extended to the experimental diffusion length. In all, the simulations supported the model proposed in analyois of the experimental data, in both of these areas. It appears that a system of randomly hopping species can demonstrate a low deviation in arrival times and sipply a amall number of surface precursors to the nucleation process. our simulations provide the means for converting the observed constant Dobs from the experimental data,

$$
<h^{2}>=2 D_{o b s} t+(h-d)^{2}
$$

to the traditionally reported surface diffusion coefficient $D_{B}$. The second term in expression 22 accounts for shifts of the nucleation site along the capillary which make the nucleation site closer to the facet edge. For the equilibrium surface concentration at $\mathrm{T}=20^{\circ} \mathrm{C}$, wo calculate $\beta=0.19 \pm 0.02$ and for $T=50^{\circ} \mathrm{C}$, we calculate $\beta=0.20 \pm$ 0.02 . The error bars reflect uncertainty in the fits of the $\beta$ vo. $1 / 6$ plots and the systematic errors resulting from the simulation algorithras. Uaing these coefficients, experimental results as shown in illustration 19 are converted to the diffusion coefficients in Table 3 , chapter 1 .

\section{Sumary:}

This chapter has presonted results of computational modeling of growing ourface systems. It was shown that computer modeling of the 
growth of a circular layer onto an elliptical substrate is feasible, and the results of these simulations were used to develop positional analysis routines. Most importantly, these positional analysis routines were largely successful in determining the nucleation site of a growth event given the time dependance of the growth reaction rate. Experimentally observed growth events were modeled in the same manner. Finally, one-dimensional hopping simulations were used to verify assumptions in tine analysis of regular growth events and provide corrections to observed diffusion coefficients. 


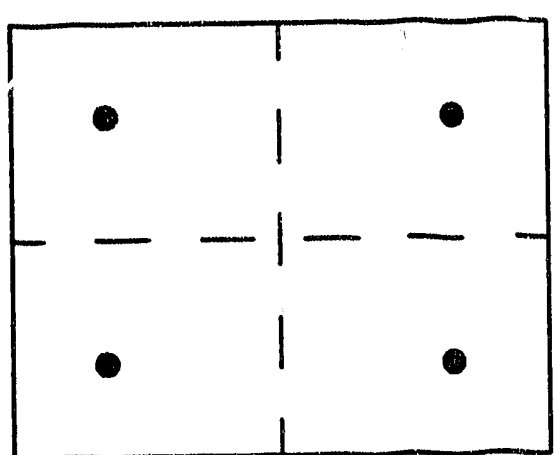

rectongle with mirror plones

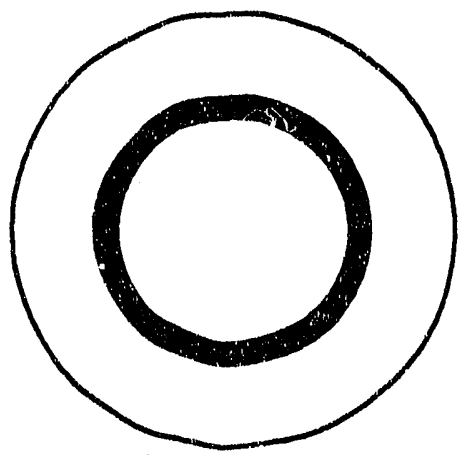

circle

illustration 20: Exanples of symetry-equivalent sites resulting from critical point analysis. For the rectangular surface, all events are mapped into one quadrant even though they could have originated in any of the four quadrants. Only radial information is accessible for events on a circular surface. 


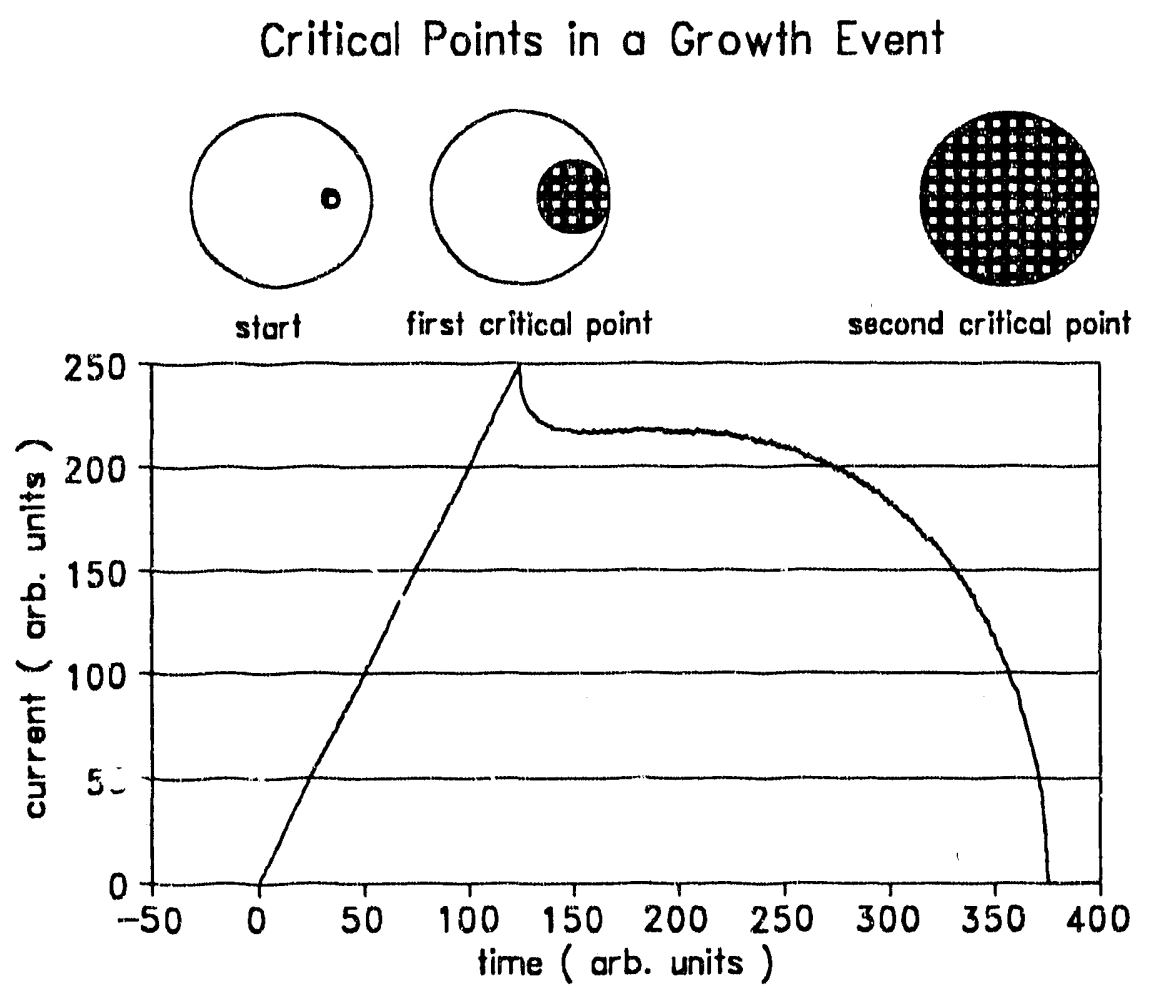

illustration 21: Time-dependent current signal of a growing twodimensional layer. The inset figures show the boundary and the new layer (dark hatching) at the three critical points in the time series. For this calculated event, the growing cluster was initially placed at a radial position half-way between the center of the cixcle and the boundary. 
Three Simulated Growth Events With Different Nucleation Sites

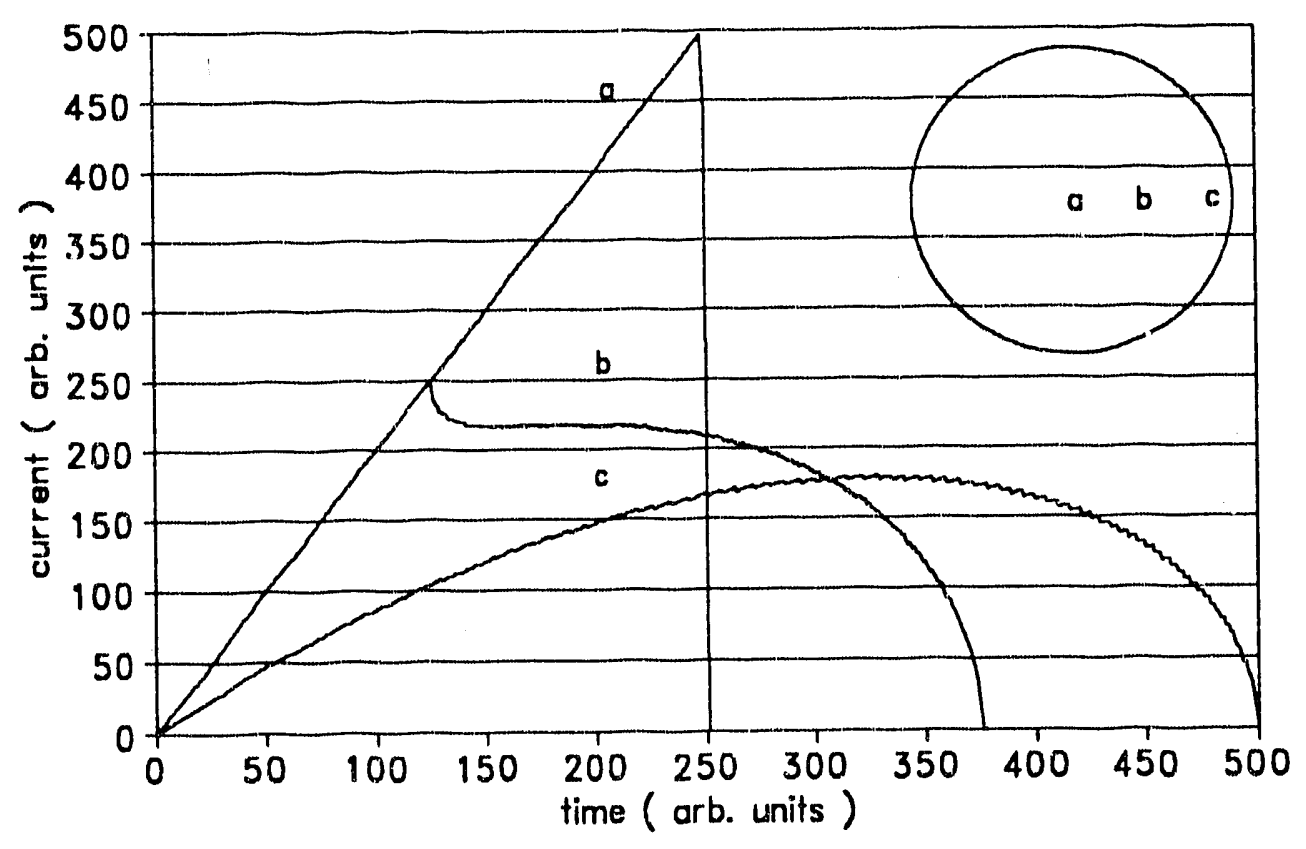

illustration 22: Three simulated growth events showing the variation in the time dependent current. Curve $b$ is from the same position as in the previous illustration. All three growth events are plotted on the same current scale and the charges under all curve are identical. For growth events starting at the boundary edge, the initial slope is exactly half of the other growth events. 


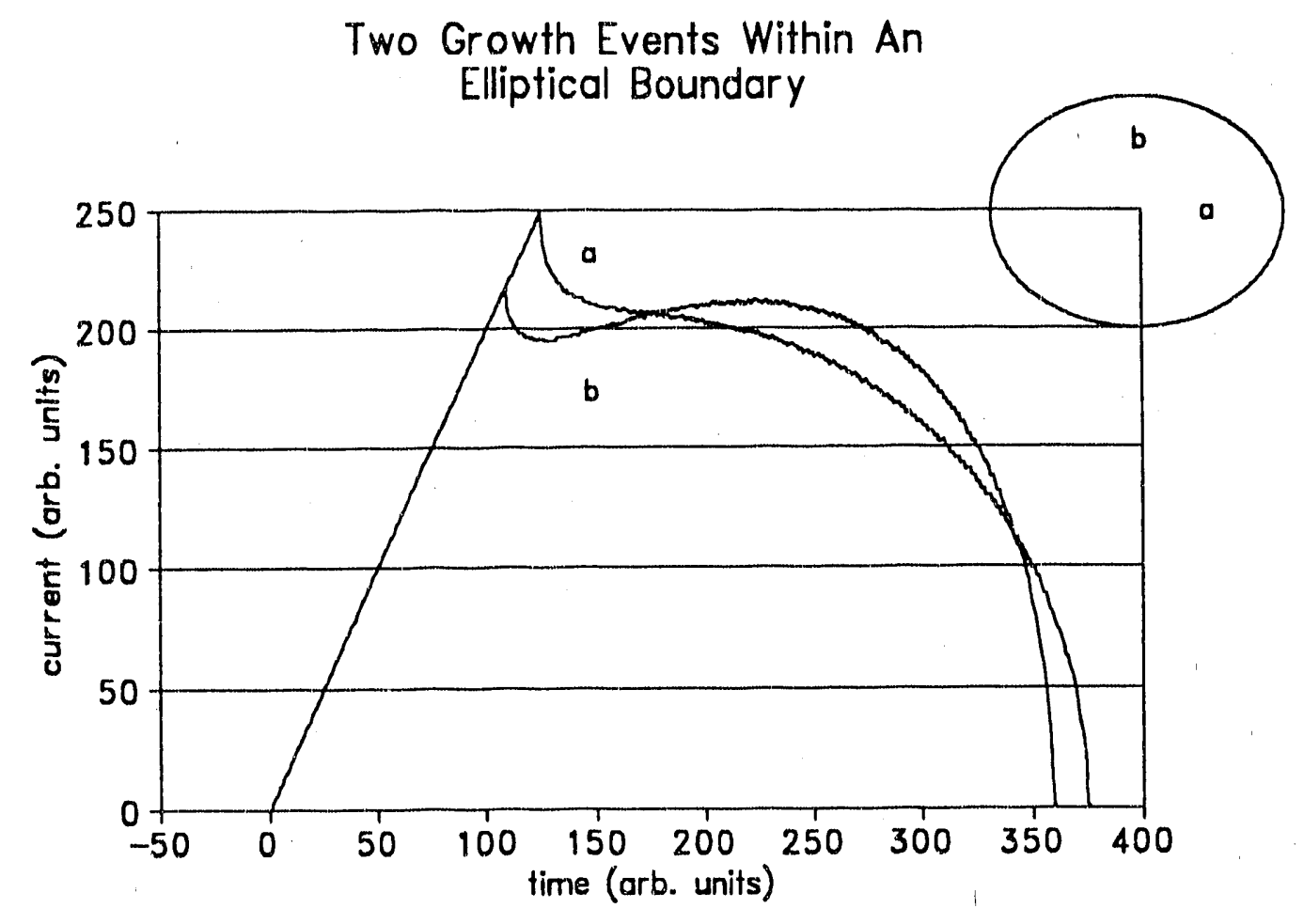

illustration 23: Growth events simulated on an elliptical boundary. The initial positions are equal distance from the centroid of the ellipse. The curves are identical until the layer has the first interaction with the capillary boundary. Curve a results from an initial position along the major axis. This curve reaches a higher maximum and ends after curve b. 


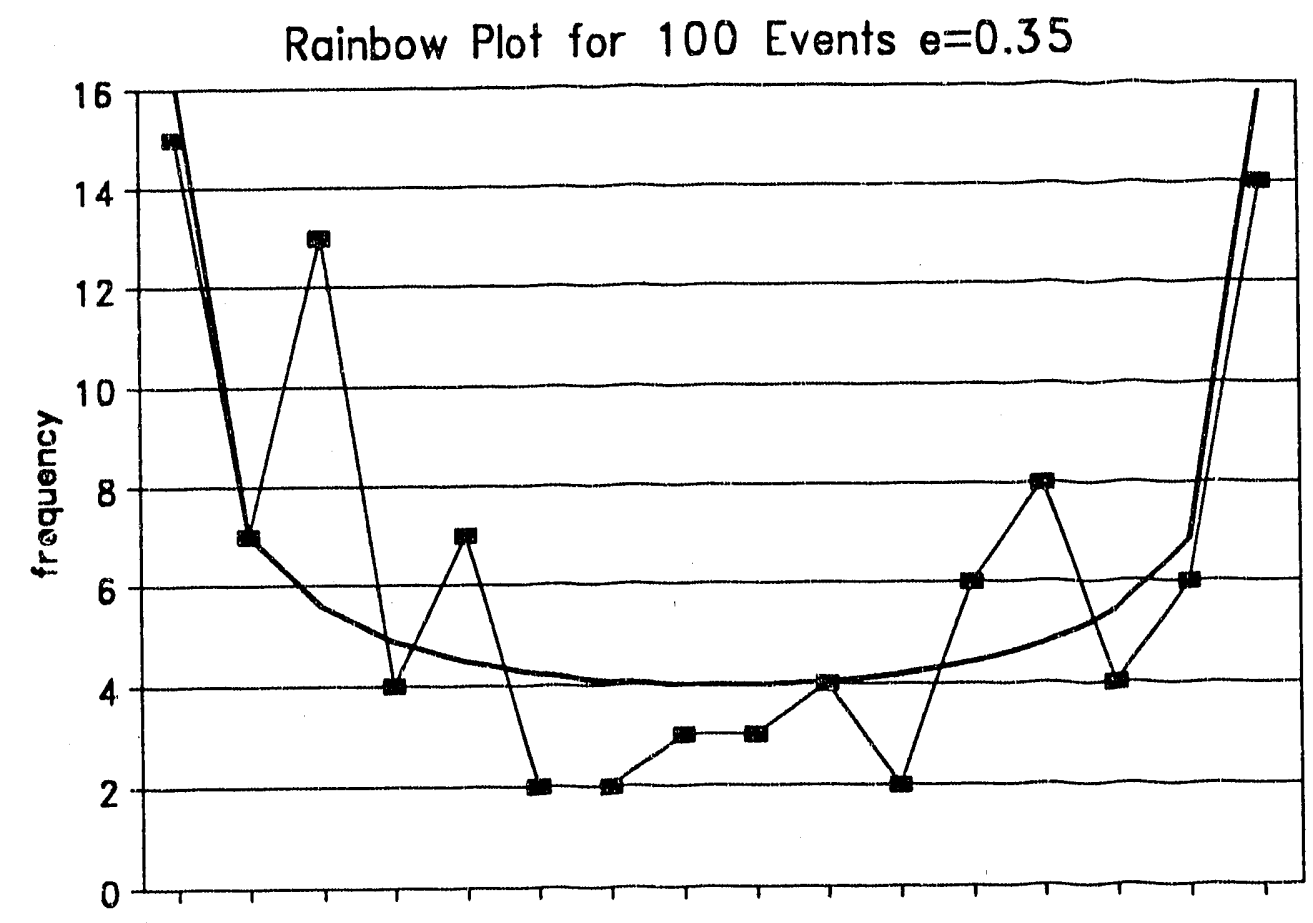

$+1+12$

illustration 24: Histogram of summed growth time $t_{1}+t_{2}$ for 100 growth events and an approximate model curve. The summed times (squares) are placed in 16 equally spaced bins for clarity. The heavy solid line is an approximate curve derived under the assumption that the line from the initial site to the final critical point passes through the origin. The left-most bin, smallest $t_{1}+t_{2}$, is from events which are close to the minor axis. The width of the "rainbow" with respect to the magnitude of $t_{1}+t_{2}$ reveals the eccentricity of the boundary. 


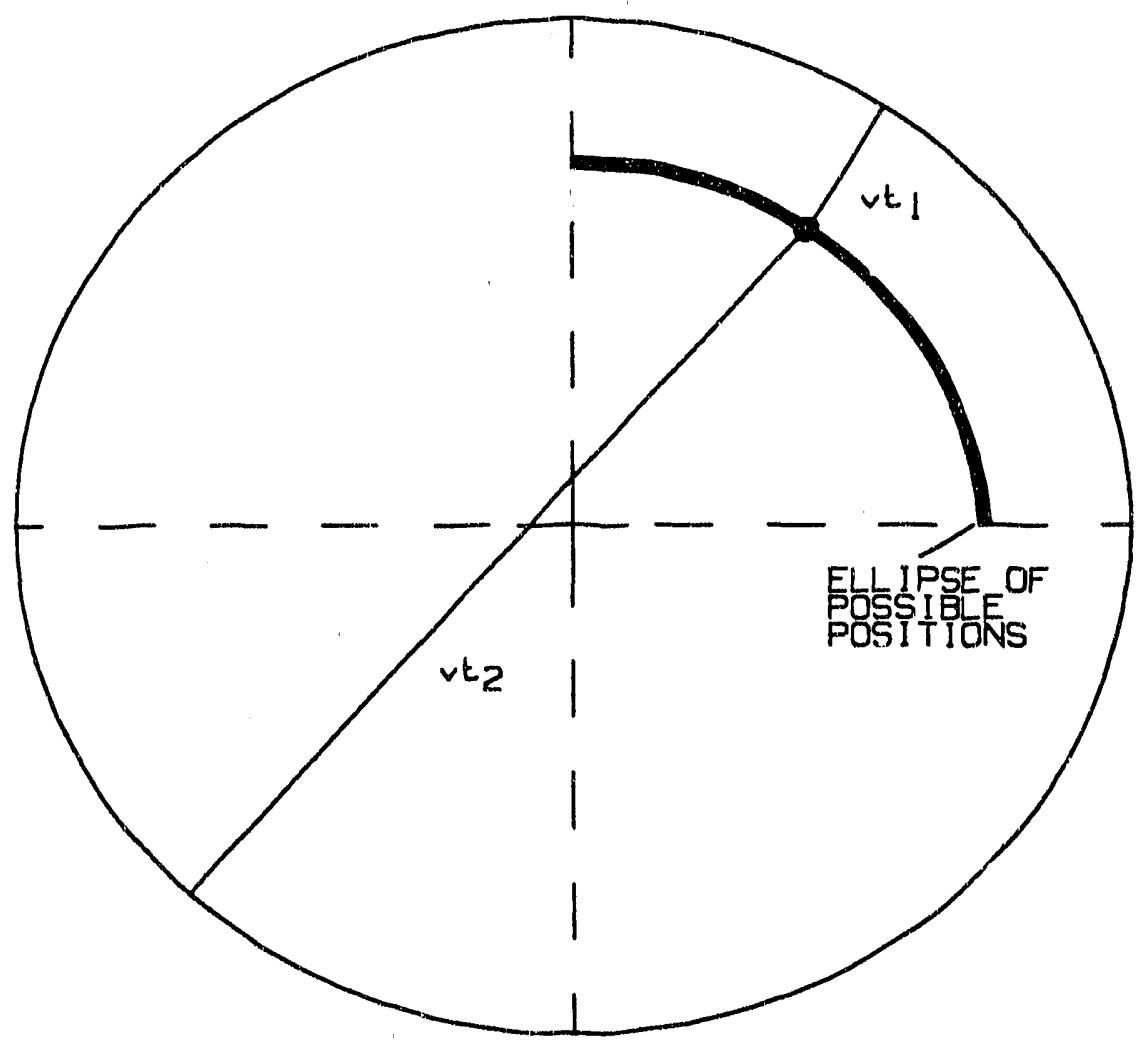

illugtration 25: Representation of the intermediate construction used in conversion of critical times into positions. The first critical time multiplied by the expansion rate generates a strip of potential initital sites. The distance which results from the second critical tine determines the nucleation site from the set of potential sites. 
Initial and Calculated Positions $e=0.35$

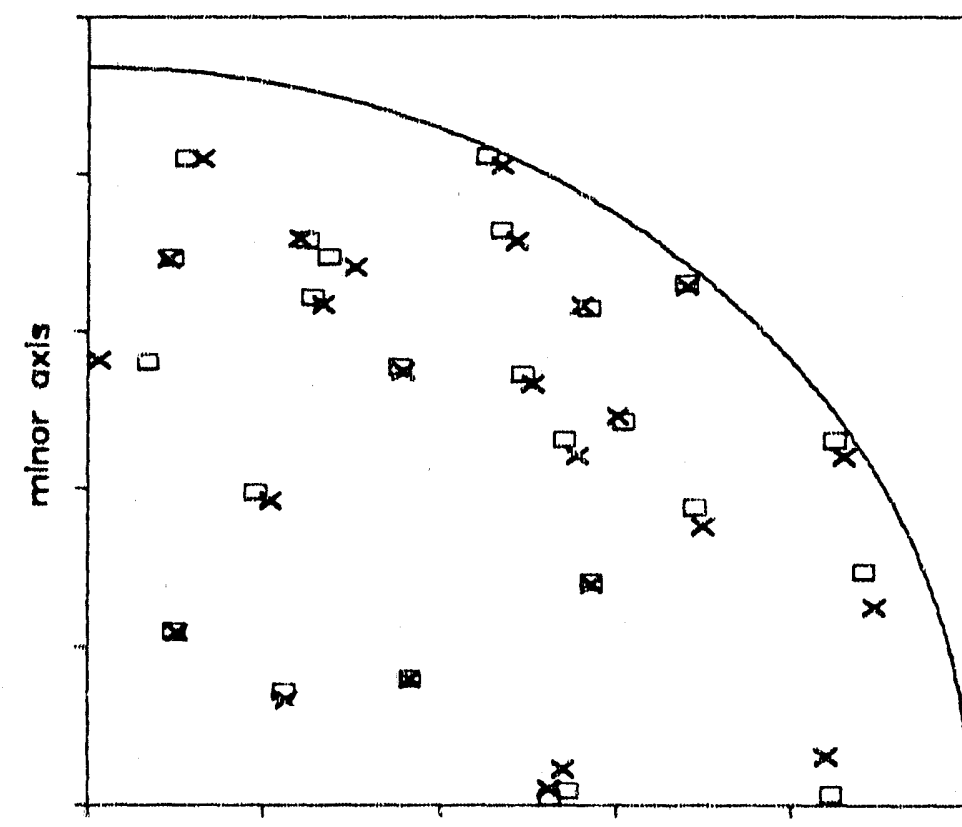

$x$
initiol
$\square$
calculated

major axis

illustration 26: Comparjoon of initial positions for simulations and calculated positions based on the growth events. A set of 25 poeitions selected at random on the surface is shown here. The mean displacement between initial and calaulated position is $800 \mathrm{~nm}$ for a capillary disuneter of $76.2 \mu \mathrm{m}$. 
Initial and Calculated Positions $e=0.65$

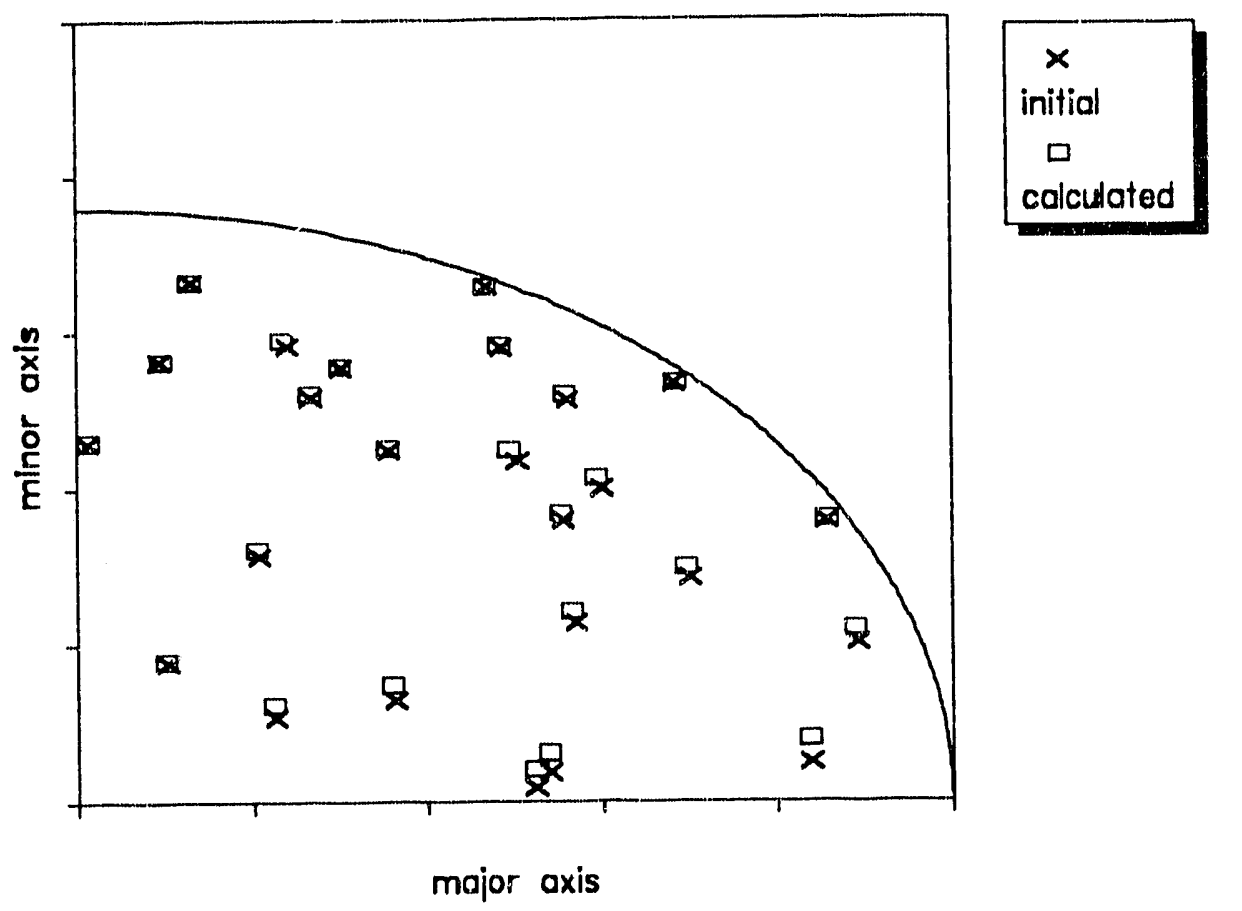

illustration 27: Comparison of initial positions for simulations and calculated positions based on the growth events. A set of 25 positions selected at random on the surface is shown here. The mean displacement between initial and calculated positions is $540 \mathrm{~nm}$ for a capillary diameter of $76.2 \mu \mathrm{m}$. 


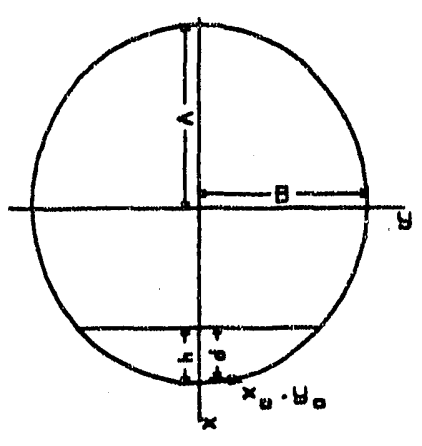

a)

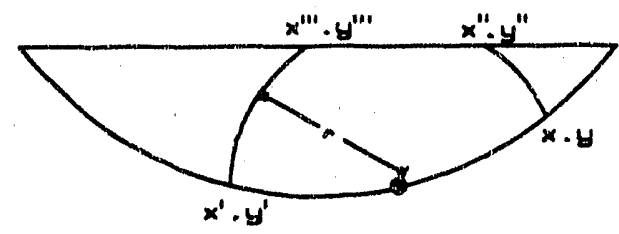

c)

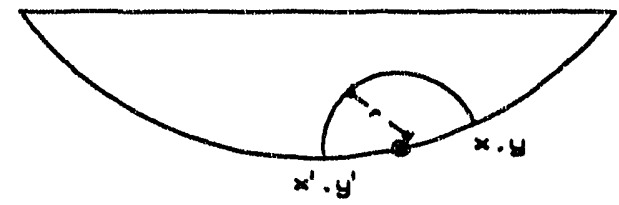

b]

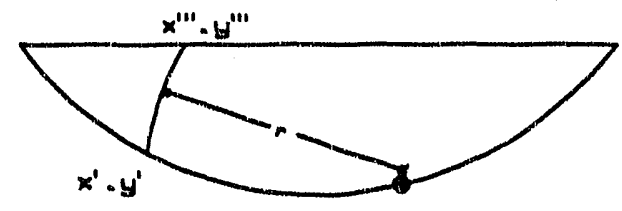

d]

il.Iustration 28: sketch of elliptical boundary, active surface area, and the three growth regimes in this system. The active sector shape is shown in a with total sector width $h$, and distance from facet edge to nucleation site d. The coordinate axes; major axis A, and minor axis B, are shown for reference. The layer in the first growth regime has two boundary intersections and $i s$ shown in $b$. This regime holds until the radius of the new cixcular layer, $x$, is equal to or greater than $d$. At this point, growth regime 2 is entered $c$, followed by growth regime 3 , d. The third growth regime is eliminated when $d=h$. 


\section{Model Curves for Different Eccentricities}

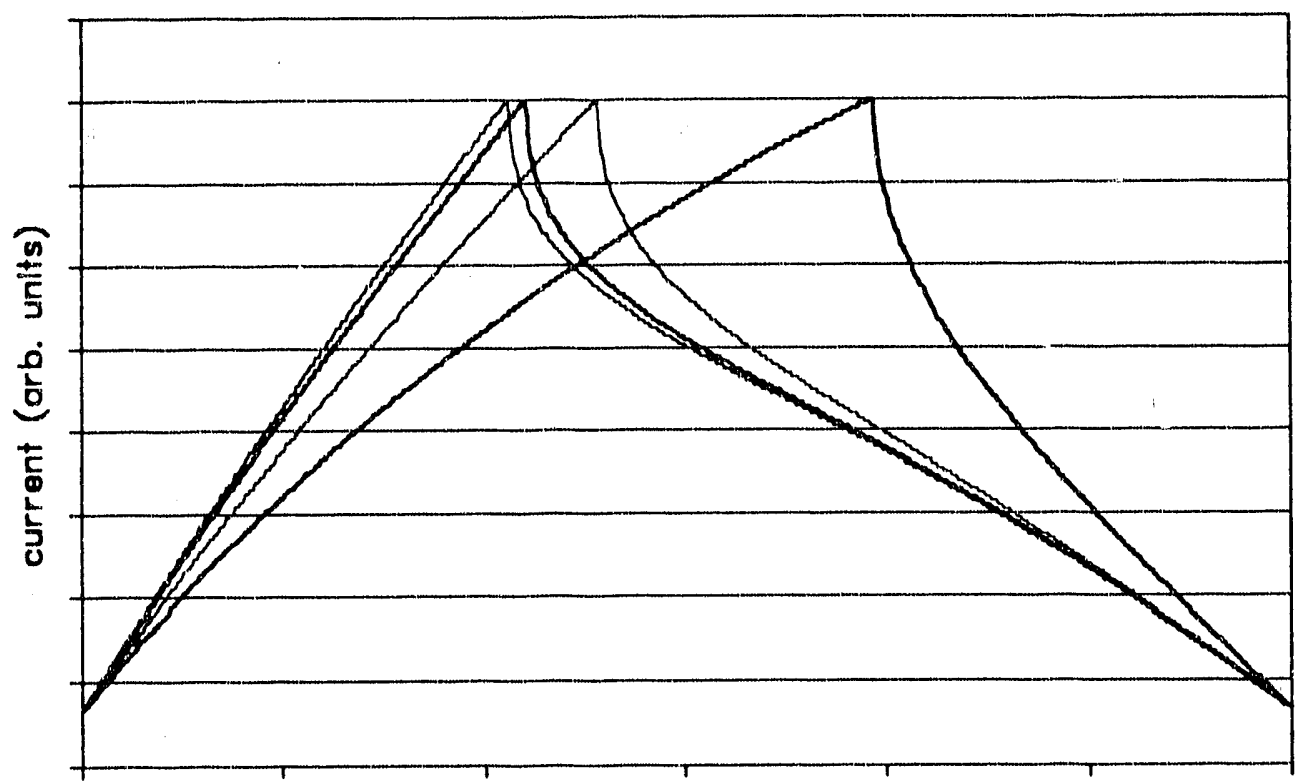

time (orb. units)

illustration 29: Four simulated events originating on boundary with varying eccentricity. The events are for surface regions with eccentricity; $0.0,0.3,0.6$, and 0.9 . The event on the circular boundary peaks first, followed by the event on $a=0.3$ boundary and the others with the latest peak from the $=0.9$ boundary. These curves are plotted on arbitrary charge scales and time scales with respect to each other to emphasize the differences resulting from boundary eccentricity. The most notable difference is that the eventis on the more eccentric boundaries show a significant deviation from linearity. A secondary effect is the shift in the peak position with respect to the total growth time, this effect is exaggerated by the normalization conditions shown here. 
Model Curves for Different Initial Positions

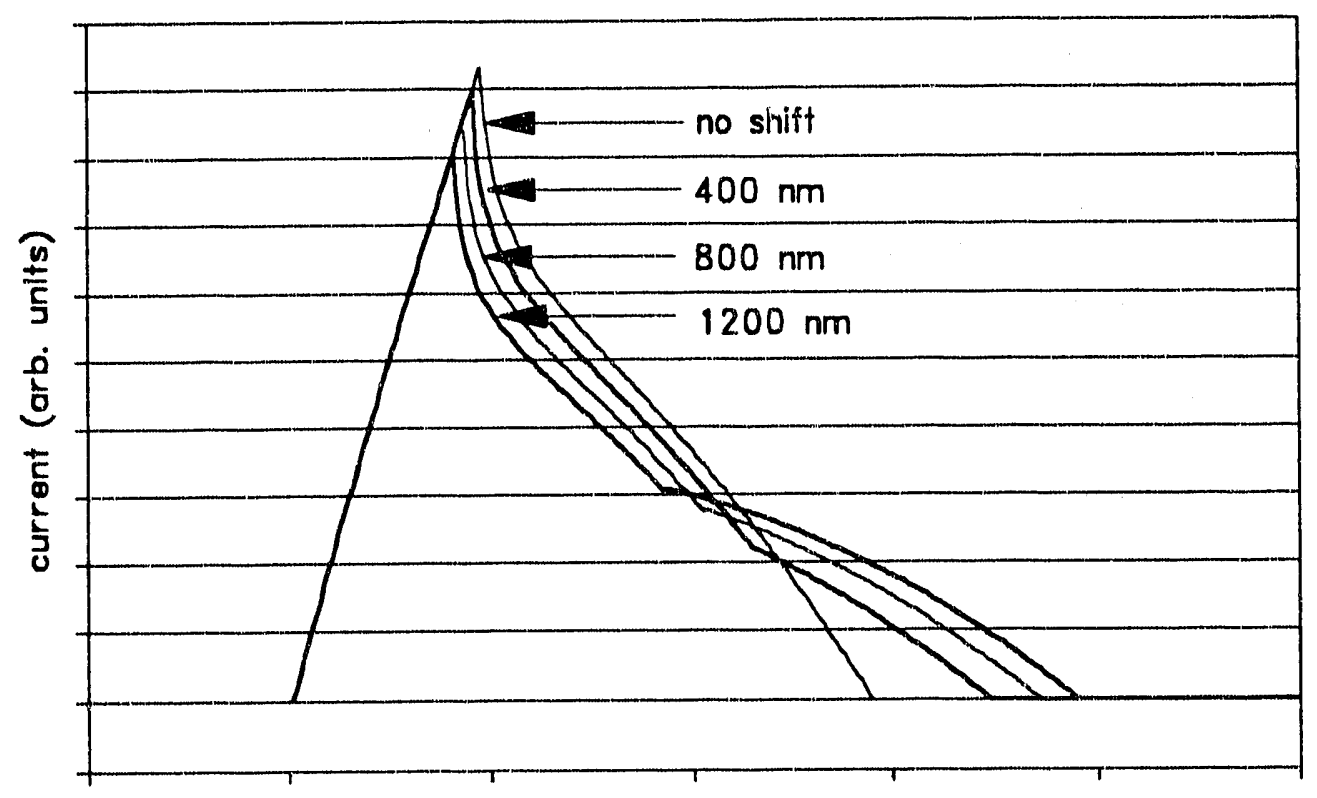

time (arb. units)

illustration 30: Four model curves for different values of $h-d$. The more an event is shifted toward the facet edge, the earlier the peak current is reached. For no shift, the transition into the third regime is not observed. The shifta plotted here bracket the approximate range in shapes observed over a number of experimental observations. 


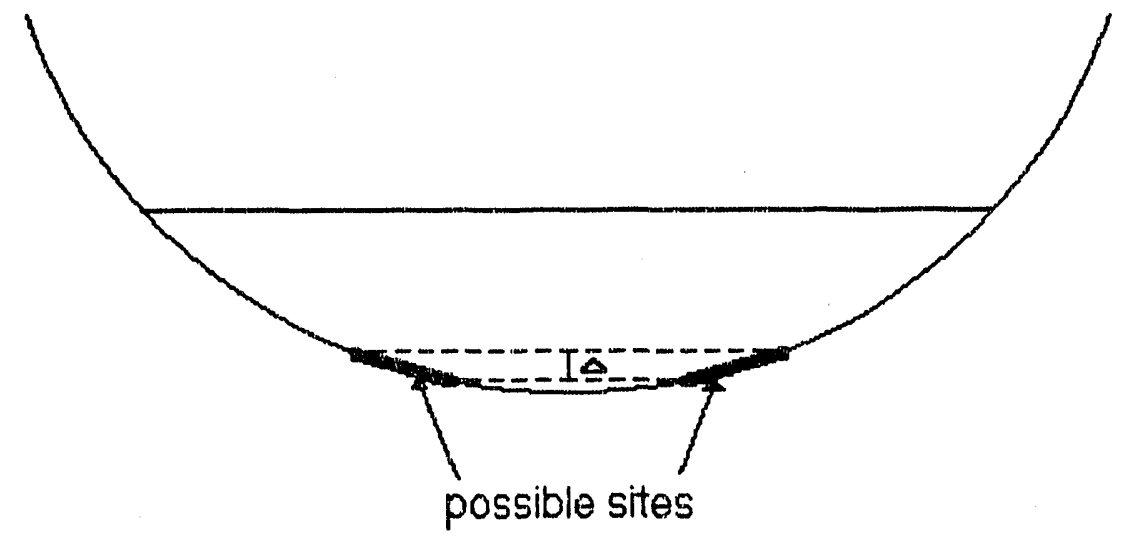

illustration 31: The impact of uncertainty in $d$ on the position determination. For the experimental data, the shift (h - d) was vary small when compared to h. This makes errors in d map onto large portions of the circular or elliptical boundary. 


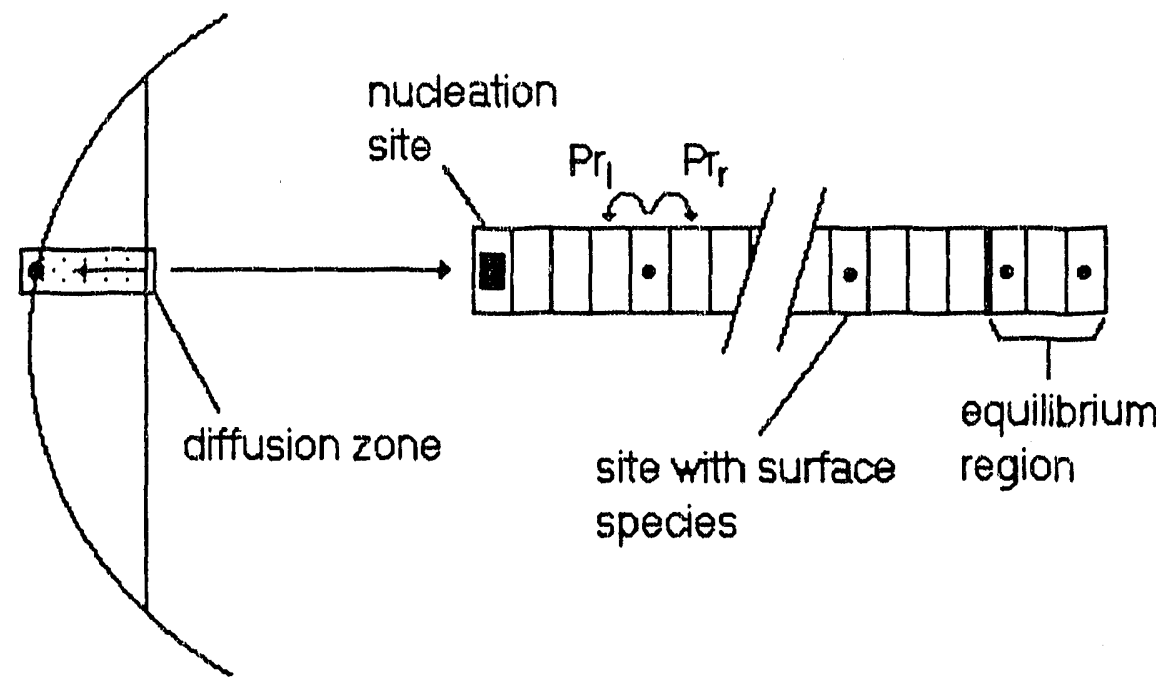

illustration 32: Methodology of surface diffusion simulations. The surface is modeled as a narrow diffusion zone, and local periodic boundary conditions allow direct extension to a one-dimensional simulation. At left is a schematic of the array used for the simulation. The concentration in the equilibrium region is exaggerated for clarity. The probabilities of a hop to the left $\left(P_{1}\right)$ and a hop to the right $\left(P r_{Y}\right)$ were half of the total hop probability. 


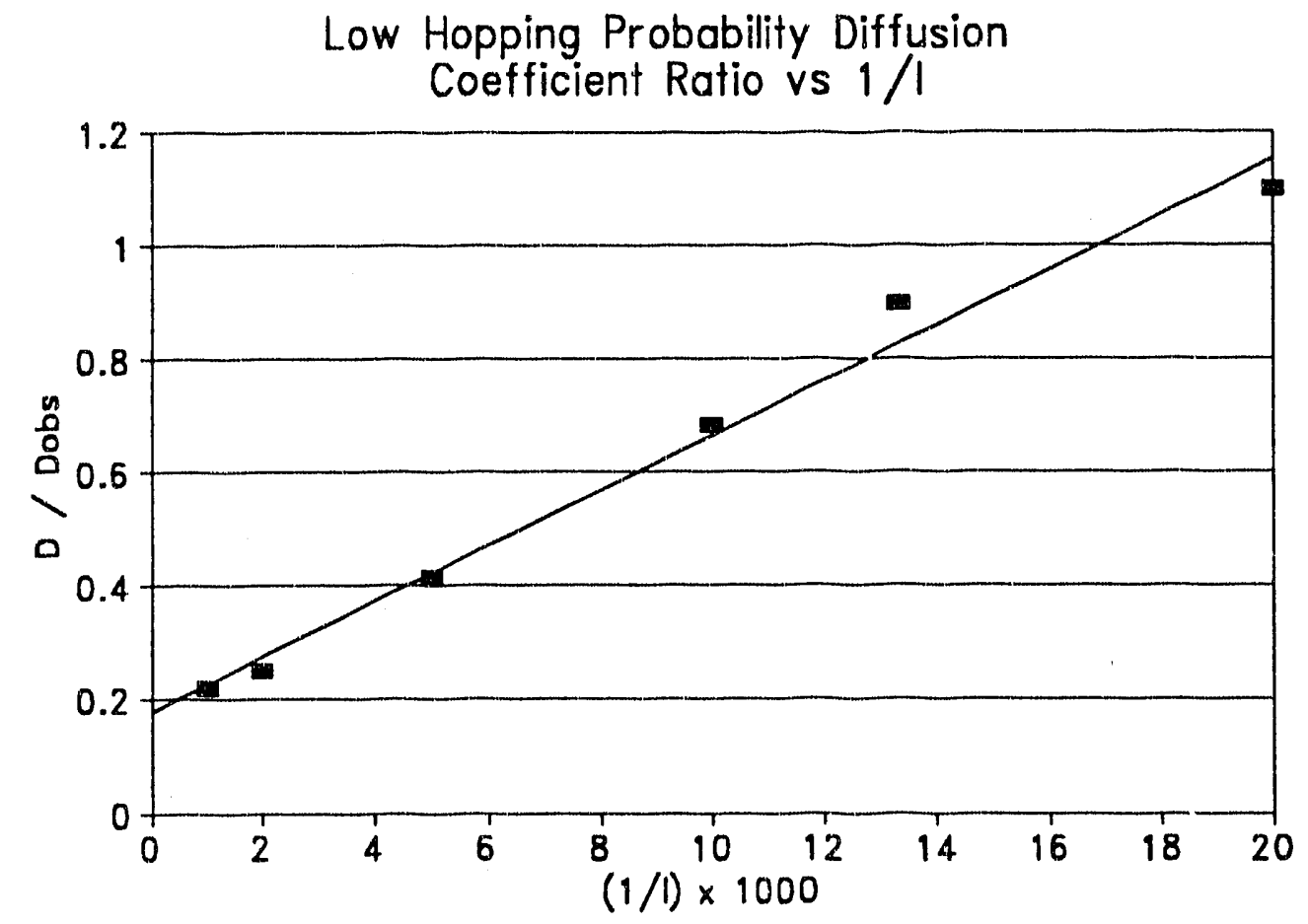

illustration 33: Results from a set of diffusion simulations. The equilibrium region for these simulations is from the $T=20^{\circ} \mathrm{C}$ results of the a.c. impedance studies. The best-fit linear regression line is shown; $r^{2}=0.986$. 
CHAPTER 3

SCANNING TUNNELING MICROSCOPY AND SPECTROSCOPY OF REALISTIC SURFACES Introduction:

The scanning tunneling microscope (STM) introduced by Binnig et al.52 in 1982 has led to an explosion in studies of local microscopic surface structure. The STM and a force-based analog, the atomic force microscope (AFM 51 , allow study of ourface physical and electronic structure on atomic length scales. The STM and AFM are actually quite flexible instruments with respect to surface feature resolution. our use of a STM has focused on phyaical features less than a nanometer to several tens of nanometers in size. The sTH allows study of physical and electronic defects that are present on realistic surfaces in ange of environments. Surface defects and their effect on local electronic and physical structure are the area of interest of this work. A special emphasis has been placed on developing techniques which can make independent, simultaneous measurements of the electronic and physical etructure of surfaces on the nanometer length scale.

The basics of scanning tunneling microscopy will be discussed here. There are several reviews of this topic that can provide additional information $57-62$. Historically, electron tunneling physics has been investigated through the use of solid state devices 63,64 . The solid state devices attained a stable tunneling current through the use of large contact areas. The earliest stable solid state tunneling devices have been attributed to Esaki65. These devices consisted of 
narrow semiconductor pn junctions. This work spurred interest in the fabrication of devices of metal strips separated by a thin insulating layer, and semiconductor/metal tunneling devices. Fisher and Giaever 66 are credited for constructing the first stable metal/insulator/metal junction. Their work and the work of others used very low junction temperatures, on the order of a few degrees Relvin.

Vacuum electron tunneling was first observed in field emission studies using sharp metal tips. A review of work in this area has been witten by Good and Mijllex 67 . It was not until the work by Binnig et al.52, however, that a stable scanning vacuum junction had been prepared. These junctions were observed to be stable over a range of temperatures, up to and exceeding room temperacure. Scanning tunneling junctions were fabricated from a very sharp metal tip and a conducting substrate. Piezoelectric devices capable of precise positioning held the tip over the sample at distances on the order of nanometers. The sharp tip allowed positioning and tunneling over very mall surface areas. This sharp tip is the key to the use of these junctions as devices capable of measuring the structure of microscopic surface features.

The STM bases its operation on the elastic tunneling of electrons. The basic one-dimensional electron tunneling problem applied to the STM has been treated theoretically by Tersoff and Bamann 68 under the Wentzel-Kramers-Brouillin approximation by the Bardeen 69 tunneling formalism. When two surfaces with delocalized electronic states approach one another, the exponentially-decaying evanescent wavefunctions from each surface can overlap at short separations. 
Although the local potential barrier between the two surfaces is on the order of 4-5 ev in vacuo, it is possible for electrons to tunnel through this classically forbidden region. Illustration 34 provides a pictorial representation of the electronic structure of the junction. Under these conditions, Tersoff and Bamann have shown that the tunneling current is:

$$
i_{t} \propto v_{B} \cdot \rho_{t}\left(E_{f}\right) \cdot \rho_{B}\left(E_{f}+e v_{B}\right) \cdot \exp \left[-\frac{s \cdot \sqrt{8 m \phi_{e f f}}}{h}\right]
$$

Where $\rho_{t}\left(E_{f}\right)$ is the density of tip states at the Fermi energy, $\rho_{g}\left(E_{f}+\right.$ $e V_{s}$ ) is the density of saniphe states at the Fermi energy plus the oample bias and $\phi_{\text {eff }}$ is the effective tunneling barrier. Their derivation assumes a tip with spherical symmetry and low sample bias comparad to the barrier height. A subsequent treatment by chen 70 has extended their approach to a tip of parabolic symmetry, with qualitatively aimilar results.

Experimentally, scanning tunneling junctions are operable in two modes. In the first, the scanning tip is moved in space without regard to the presence of the sample. This is the "open-10op" mode. Typically, the tip is scanned roughly parallel to the plane of the surface over a small, square, surface region, that may be tens to a few hundred of angstroms on a side. The tunneling current fluctuates during the scan and is recorded as a function of tip $(x, y)$ coordinate. The fluctuations in tunneling current can be correlated to electronic and physical surface structure through equation 23. This mode of oferation is often called constant-height or fixed-height, a slight misnomer. In the second mode of operation, the tip position is controlled by an 
active feedback loop designed to maintain a constant quantity of a measured signal. The most common input signal for the feedback circuitry is the current flowing through the junction. When the tip is scanned over the surface, the vertical deflections required to maintain the feedback parameter (e.g. the tunneling current) constant are recorded. These deflections can be roughly correlated to the surface topography. In the limit of constant surface composition and tunneling transmission factors, the apparent topography is directly related to the physical structure of the surface. This mode is often referred to as the constant-current or fixed-current mode. More generally, the feedback method can be described as the "closed-loop" mode.

Along with scanning probe experiments at a constant junction bias, recently low temperature "spectroscopic" techniques have also been applied by several researchers to these experiments performed at room temperature. Hamers 60 has published a review of recent work in scanning tunneling spectroscopy (STS). At room temperature, thermal energy has a significant impact on the electronic state occupancy of electrons close to the Fermi level. This broadens the energy resolution of tunneling experiments to roughly $\pm 2.5 \mathrm{kT}^{63},( \pm 65 \mathrm{mv}$ at room temperature $)$, preventing the observation of low-energy inelastic vibrational transitions, for example. However, spectroscopic techniques have been successfully applied to study electronic band structure at the surfaces of semiconductors and metals. Spectroscopic information can be acquired in both one-dimensional and two-dimensional experiments. The smallsignal conductance $(\mathrm{di} / \mathrm{dV})$ at constant bias is commonly used in STS following the work performed on solid-state devices $60,63,64$. 
The small-signal conductance is experimentally measured in one of two ways. One type of conductance measurement attempts to hold the tip and sample at fixed-height while tho bias is linearly ramped over the range of several ev. The measured $i(v)$ curve is differentiated with respect to bias to yield the conductance as a function of bias. The conductance can also be measured directly with lock-in amplifier techniques under conditions of constant separation and d.c. current. The first type of measurement is faster than the second type of measurement; however, there are two significant problems with the technique. The bias ramp can be damaging to the physical state of the tunneling junction. Recent work has demonstrated the power of the tip to cause surface modification ${ }^{71}$. These modifications are triggered by high bias or fast voltage sweeps, and until the mechanism is understood fully, the possibility that the tip is modifying the surface during the acquisition of the $i(V)$ curve cannot be eliminated. The $i(v)$ curve method also sacrifices dynamic range at low bias and current, because of the extremely small currents passing between tip and sample at low bias. The form of (di/dV) as a function of bias is dependent on several junction parameters. In practice, the small-signal conductance is normalized by the d.c. conductance to reduce contributions from biasdependent tunneling transmission terms related to changing the effective barrier under the conditions of the experiment ${ }^{72}$. Lang ${ }^{73}$ has demonstrated that $(\mathrm{di} / \mathrm{dV}) \cdot \mathrm{V} / \mathrm{i}$ is roughly proportional to the density of states in certain systems. The true functional form is probably closer to that given by Hamers (ref. 60 page 542 ): 


$$
\frac{d[\ln (i)]}{d[\ln (V)]}=\frac{\rho_{g}\left(E_{f}+e V_{B}\right) \cdot \rho_{t}\left(E_{f}\right)+A\left(V_{B}\right)}{B\left(V_{B}\right)}
$$

where $A\left(V_{B}\right)$ is related to the integral over energy of the derivative of the junction transmission terms with respect to bias and $B\left(V_{B}\right)$ is the integral over energy of the transmission terms. starting with the result from Tersoff and bamann (equation 23 here) it can be shown under our experimental conditions that the effective barrier and the tunneling area of the junction play a major role in the ratio $A\left(V_{B}\right) / B\left(V_{B}\right)$.

The STM has been very successful in elucidating for the first time the atomic structure of many ourfaces 57,58 . Moving beyond the determination of atomic scale corrugations to the atudy of electronic properties, or larger structural features, or adsorbed molecular species has been complicated due to ambiguity in interpretation of STM images. The theoretical treatments summarized above are valid only for physically and electronically simple systems and under very low applied bias. Study of many realistic surfaces in air has revealed that most surfaces retain a morphology that is dominated by bumps and hillocks $61,62,74$. It is difficult to determine where the substrate surface ends and contaminants or intentionally deposited species begin. At this point in the development of the subject, a better understanding of how the microscope actually operates and the application of additional complementary techniques are both required in order to make progress in the development of the STM as a chemicaljy surface-sensitive tool.

Our research has focused on developing instrumentation that is optimized for acquiring simultaneous images of topographic and 
spectroscopic information. Once the raw experimental data has been obtained, it must then be analyzed quantitatively in a number of ways and visuaized in an insightful manner, so these computational. technigues have boen developed an well in this research program. our experimental apparatus measures the spectroscopic information at a aingle bias while scanning over the surface. One advantage of this approach is that numerical differentiation of an often noisy signal is avoided. Another advantage is that the tunneling junction is not axposed to the high voltage-Bweep rates required to obtain currentvoltage curves "on the fly". A significant experimental disadvantage of our approach is that the time to accuire a true conductance apectrum over the surface is longer because an entire image needs to be acquired at each bias.

By combining simultaneous images of topographic and spectroscopic information, local changes in the small-bignal conductance can be correlated with the apparent surface atructure 75 . This approach has allowed us to obtain additional inaight into the electronic properties of platinum and graphite surfaces in air. In the present work, the length scales of interest are on the order of nanometers to tenn of nanometera. Fox imaging in this window, a reusable type of tip was developed for use along with more traditional sTM tips. The rest of this chapter covers the basice of the microscope constructed during the thesis research and experimental observations of avaporated gold films, polycrystalline platinum, and highly orianted pyrolytic graphite. 


\section{Experimental:}

\section{Apparatus and Instrumentation Overview:}

The hardware for the scanning tunneling microscope consists

of four basic parts: mechanical mounting and vibration isolation apparatus; piezoelectric scanning system; tip and sample mounts; and customized electronics for microscope control and data acquisition. The main mounting apparatus includes two jayers of acoustic foam shielding and is suspended by elastic cords. Two piezoelectric devices, one composite scanning drive with three elements and one single-element drive, were employed. The tips were about $1.2 \mathrm{~cm}$ long with a mounting post at one end. Samples were about $5 \mathrm{~mm} \times 5 \mathrm{~mm}$ in size. The electronics for the instrument contained three subsystems; scanning and experiment control, experiment monitoring circuitry, and data acquisition system. Most of these aspects were developed as part of the thesis work.

Mounting and Isolation Bardware: Illustration 35 depicts the basic mounting system of the microscope. A plywood board (chosen for its lossy acoustic transmission properties) supported by a unistrut frame serves as the mounting plane for a small vibration-isolation table. The plywood board is suspended by elastic (bungee) cords from a unistrut framework hung from the ceiling. The mechanical resonant frequency of this base plane when the apparatus is fully installed is on the order of one Hertz. The resonant frequency of the vibration-isolation table (Newport model Ts.-23) is 
between $20 \mathrm{Bz}$ and $50 \mathrm{Bz}$ depending on the pressure of gas in the table supports. The frequency mismatch between the plywood plane suspension system and the vibration isolation table effectively eliminates propagation of building vibrations to the experiment. This suspension design borrows from Bansma 76 and others. Acoustic isolation is provided in two ways: a plexiglass enclosure that reflects sound waves and waffled composite fiber foam mounted on the underside of the plywood board and on the inside of the plexiglass box. A second layer of acoustic isolation is provided around the microscope itself by a amall. plexiglass box with foam on its inside walls. Each acoustic isolation layer provided about $20 \mathrm{~dB}$ of attenuation of laboratory noise. The overall vibration and acoustic isolation outlined here reduced the effects of laboratory noise sources (in a laboratory on the second floor of a building) so that they yielded interference on the ordex of the electronic noine in the system.

\section{Scan Elements and configuration:}

The piezoelectric elements used in this microscope were fabricated by Burleigh Inztruments, Inc. A three element PZT Aligner/Translator model PZ-81 was used to generate the scans across the surface and to control the tip position in "closed-loop" experiments. A basic PrT Pusher model PZ-40 was employed for coarse positioning of the tip and sample. Both piezoelectric devices were constructed of "hard" piezoelectric ceramic, providing relatively small deflections and very lineax, non-hysteretic, behavior. The roll-off frequency for smallwoignal response is at $5 \mathrm{kgz}$ for both devices. These elements were 
configured in two ways, parallel (illustration 36) and coaxial (1llustration 37). The parallel configuration was used for most of the work discussed here. The coaxial configuration was developed to provide lower drift rates after microscope assembly and simplify sample handling .

The parallel configuration used two MACOR ${ }^{\oplus}$ ceramic slabs as mounts for the piezolectric drive elements. MACOR was chosen because of its near-zero coefficient of thermal expansion at room temperature and lossy acoustic properties. One $3^{\prime \prime} \times 3^{\prime \prime} \times 1 / 2^{\prime \prime}$ MACOR ${ }^{\circ}$ slab held the scanning PZ' in the center and was rigidly mounted to the top of the vibration isolation table. The coarse adjustment single element PZT was mounted in one corner of the slab. These components were mounted on the reference (fixed) slab to reduce motion caused by residual stresses in power cables. The second ceramic slab was positioned on this reference frame by 6 ball-bearing contacts. Coarse positioning was accomplished in the plane of the 8 lab by three 80 threads-per-inch (tpi) screws. The gross separation between the tip and sample was changed by tilting the movable slab by lengthening the coarse adjust screw or the coarse adjust PZT. Tilting the slab produced a mechanical disadvantage of $3: 1$ with respect to the length of a 80 tpi screw and the extension of the coarse PZT.

The coaxial configuration used a single mount to hold the entire microscope assembly. The reference MACOR slab from the parallel configuration was used without modification. In the coaxial configuration, the coarse PZT is held within the scanning PZT. A brass fitting with a micrometer mount was screwed onto the threaded end of the 
coarse PZT. The micrometer was in turn mounted on the back side of the scanning PZT with an aluminum mount. The vertical position of the coarse PZT within the scanning PZI could be adjusted with the micrometer. The sample in this configuration is held in a ceramic fitting in a cap which screws onto the end of the scanning PZT. Whe miniaturization resulting from this design reduced translational drift rates by one order of magnitude.

rip and sample Nounting:

The tips and samples for the microscope were designed to work as plug-in parts in the parallel configuration. The tips were $1.2 \mathrm{~cm}$ in total length with a plug of about $0.4 \mathrm{~cm}$ of 18 gauge wire. The plug mated with a gold receptacle attached to the scanning PZT by a fiberglass mount plate. The samples were mounted on top of a gold receptacle identical to the tip mount. The mounting ourface was circular with $0.10^{\circ}$ diameter. By witching the basic mounting connector, samples on an 18 gauge post could also be used.

When the configuration was changed to the coaxial configuration, some mechanical changes were implemented in the tip and sample mounting. These changes eliminated the springy gold receptacles which may well have been contributing to start-up mechanical drift. Flat amples with thicknesses comparable to a glass microscope slide could be mounted to the MACOR holder within the sample cap. Samples of this type were held in place by a low contact pressure fit by a copper-beryllium spring. Thin metal samples could be used by placing a glass microscope slide of the same aize on the back of the sample. Post-type (18 gauge) samples 
and tips were held in place by a collar assembly. This provided a more rigid mounting point for both. Tips were mounted in the microscope by affixing the collar holding the tip to the end of the coarse pzT via a high-power magnet.

Electronics:

The scanning electronics were designed to take input deflections from an external source in the form of analog inputs in $x, y$, and $z$. The input signals were converted into drive signals which produced translation of the tip with respect to the sample. The scanning PzT consisted of three independent elements mounted to a $I^{\prime \prime}$ diameter drive ring. The elements were mounted at $120^{\circ}$ separation. Ey proper scaling and combination of the input signals, the three elements could be used to achieve vertical translation $(z)$ and horizontal translation ( $x$ and y). A symetric application to all three drive elements produced the vertical translation. Two asymetric combinations produced two orthogonal tilting modes in the plane of the tip mount. These tilto become deflections at the end of the $1.2 \mathrm{~cm}$ long tip. Illustration 38 shows the location of the individual piezoelectric elements, the symmetric drive mode, and one of the asymetric modes. An analog computer was constructed from operational amplifiera to perform the conversion from input $x, y$, and $z$ to the drive signals for elements 1, 2, and 3. Bigh voltage operational amplifiers, Burr Brown 3582J, were used in the final stage to produce high-speed signals of up to $250 \mathrm{~V}$. A schematic of the analog computer and high voltage drive circuitry is given in appendix $D$ (figure D3). 
Electronics to monitor the tunneling junction and control the position of the tip are also shown in appendix D. Current measurement was accomplished by a dual operational amplifier. Half of the amplifier converts the nA tunneling current into a mV signal while the other half drives a guard shield for the tip's electronic lead. A triaxial wire is used as described in chapter 1 for the current measurements. The monitoring circuitry produced a suitable signal for external acquisition and compared the tunneling current signal to a reference signal. The difference between these signals was converted into an applied deflection of the appropriate sign to the tip position. An integrating circuit of adjustable rise-time was used to reduce noise in the tip height control circuit.

Computer and Software Interface:

The final major electrical hardware component is the control computer and its hardware interface to the scanning and monitoring circuitry. The computer controlled the experiment by signals to the electronics of both añalog and digital form. A Metrabyte DDA-06 board was used for these signals. The analog control signals from this interface board were used for $x$ and $y$ deflections and for the control signal to the coarse PZT. To prevent contamination of junction control signals by scan signals, a separate Metrabyte DAC-02 interface board provided the analog signals for fixed-current level and junction bias. signals from the experiment in analog form were acquired by a Metrabyte DAS-50 analog-to-digital converBion board. An IBM-compatible DELL model 
310, with $80386 \mathrm{CPU}, 80387$ math coprocessor and $8 \mathrm{Mb}$ of RAM, was used for the experiments.

The software to run the experiment was written as part of the research project. Software for a STM must address several issues and also be capable of handling the large data seto which result from acquiring the image. Several computer programs were written to accomplish the tasks of acquiring and analyzing the data. For an IBMtype computer running MS-DOS, the small program approach is the most efficient due to the memory model employed. A menu group within the Microsoft Windows 3.0 environment was created to facilitate access to the microscope software. The windows environment provided a pseudomultitasking environment where several programe related to the operation of the microscope could be run simultaneously. Communication of data between programs was accomplished by reading and writing temporary files on a RAM disk. Programs to accomplish microscope control, data acquisition, and data viewing will be outlined below. The actual code for these programs is quite substantial and is provided as supplemsntary material rather than being included here as an appendix.

The basic microscope control program was coded in Microsoft QuickBasic 4.0. BASIC was used for the system control because of the ease of operation of interface boards in this programing language. This program offers a menu of choices for current/bias control, coarse approach, setup of scans over the surface, and two display routines. Each choice produced another menu or dialog to set up relevant parameters. The scan setup allowed the choice of operation mode (closed-loop or open-loop), time for the scan, and the iuput channel(s) 
to record during the scan. The scans were $256 \times 256$ positions with an adjustable number of samples at each position between 1 and 16 points. Data was "smoothed" by linear averaging at each point rather than by using fast Fourier transforms and frequency filters. Data were displayed as 16-color images coded for height or other appropriate junction parameters. The topographic data could also be displayed in a throe-dimensional line-scan representation. A menu built into the 16color image display allowed zooming and sub-scans of the default imaging area. Image sizes could be adjusted from $3 \mathrm{~nm} \times 3 \mathrm{~nm}$ to $120 \mathrm{~nm} \times 120 \mathrm{~nm}$ with this program. The program obtained the display data by reading scratch files on the RAM diak written by the data retrieval program. The data for each position, with multiple samples at each position, were stored in the on-board memory of the DAS-50 interface card. The interface board contained $1 \mathrm{mb}$ of local storage which relieved system $i / 0$ demands during high speed acquisition. A Microsoft FORTRAN 4.1 program was written to retrieve this data. The program read multiple data sets automatically and added the data for each position together to get a final array(s) of $256 \times 256$ points representing the average of several "scans". The data array(s) were saved as a scratch file for use by other analysis programs with a header to specify important parameters of the scan. This program also computed and stored the data for the 16-color inlages displayed by the main control program. Typical execution time to retrieve a data set of 4 samples per data point, add the samples together, calculate the 16 color image and write the raw data and the image data to temporary files, was 10 seconds. 
In addition to the control program which provided quick and simple viewing for the purpose of quality control, a program was written to do more extensive data analysis including local filtering, plane subtraction, image display, and export of one-dimensional slices of the two-dimensional data sets. This program was a mix of Microsoft QuickBasic and FORTRAN routines. The main routine was written in BASIC, while FORTRAN was used for mathematical processing of the data. Algorithms in BASIC for performing local averaging ran 5-10 times slower than analogous code in FORTRAN. The start-up menu presented data import and export options and two display techniques. Display options were similar to the control program; however, more flexibility in processing was provided. Function key menus within each display routine allowed user-input scaling, local averaging, setup and run of a plane subtraction routine, image export to binary or plotter command files, and export of one-dimensional slices of the data. One-dimensional slices could be averaged over a number scan lines, and the direction of the slice was fully adjustable in 2 dimensions. This program was used to prepare data for export to specialized graphics rendering hardware by local filtering and plane subtraction. Data files to be exported were saved to temporary files that were read by one of two application visualization system (AVS) field preparation programs. FORTRAN and in later versions, $c$, were the languages used for the AVS data preparation programs. One program was designed to comprass the data at each point to 7 bits or 8 bits (c version) based on the maximum and minimum values in the image. The other program allowed interactive operation and displayed a histogram of the image data values. The user 
selected a midpoint for the data and a window width for the data compression. Data compression to byte width allowed the image data to be understood by other computer hardware types, making it possible for a personal computer running ms-DOs to send data to a graphics supercomputer running the UNIX operating system.

Eigh quality three-dimensioval visualization of the STM images was achieved on a stardent GS-2000 computer. The graphical output of this system is shown in the color plates in this thesis. All processing of data was done in AVs, a flexible graphical visualization environment. For the topographic data, Avs was used to convert the data into a threedimensional surface which could be manipulated and viewed in real time. Data processing networks were developed within the AVS Network Editor to read data, filter data, and generate three-dimensional objects and twodimensional color maps (images). Data filtering consisted of linear subsampling of the data and cropping the data. Data which had been subsampled by a factor of 2 to 4 could be used for interactive, threedimensional visualization. Texture-mapping was used to map a color image onto the three-dimensional topographic daca. This graphical technique places color values at the vertices describing the threedimensional object. This vjoualization technique allows insightful viewing and presentation of simultaneous images of topography and conductance. Color prints of the results were generated on a kodak XI7700C color printer.

Iip construction: 
Three basic tip types were developed for use with this microscope; silver microcrystal, platinum foil, and tungsten/tungsten oxide. of these, the platinum foil and tungsten based tips provided resolution down to atomic aized features. The silver microcrystals were used for imaging surface features of larger size. The silver crystal tips were grown from a concentrated silver nitrate solution in exactly the same method as the seed crystals for the silver growth experiments were grown. The body of the tip was sealed in glass to prevent silver deposition except at the exposed platinum disk of $125 \mu \mathrm{m}$. Platinum tips were prepared by cutting platinum foil with a pair of sharp scissors. Each cut was about 2-3 m long and tapered to a narrow sheared end. These small foil wedges were mounted on a 18 gauge copper wire with silver epoxy or nickel paint. The silver epoxy provided slightly better adhesion, at the cost of an annealing step. Tungsten tips were fabricated by etching in 3M NaOE under galvanostatic conditions. The end of an 18 gauge tungsten wire was protected by a short teflon tube 77 . Etching to a neck above this sheath was accomplished by a d.c. current of 50 to $70 \mathrm{~mA}$, at voltage limit $25 \mathrm{v}$. The neck was broken, leaving a sharp tip, with a square wave current of $\pm 5 \mathrm{~mA}$ with voltage magnitude 2 $\mathrm{V}$ and dwell time $3 \mathrm{~ms}$. This finishing step leaves a doped thin layer of tungsten oxide over the end of the tip 78 . The tungsten oxide prepared in this manner is a non-stoichiometric bronze, $(\mathrm{B}, \mathrm{Na})_{x<1} \mathrm{WO}_{3}{ }^{79}$, hereafter referred to as "Wox". All tips were checked for gross shape with a $100 x$ microscope. Usually, tips which appeared very blunt did not work in the microscope, making gross checks worthwhile. 


\section{Sample Preparation:}

The samples prepared for this work were mounted on gold sockets. The evaporated gold films were deposited on glass microscope slides to thicknesses of 1000 in the Majda 80 research lab. The glass microscope slides were broken into small shards, which were mounted on the socket with epoxy. A strip of nickel paint was used to provide electrical contact between the gold film on the top of the glass microscope alide and the gold socket. After assembly, the gold samples were cleaned by rinsing with $n$-hexane. Platinum foil samples were spot welded onto an 18 gauge platinum wire. An entirely platinun part allowed flame cleaning and quenching in concentrated BCl. After the acid clean, the platinum assembly was heated and quenched in ultrapure water to remove residual sodium. The wire was plugged into a gold socket during microscope assembly. Bighly oriented pyrolytic graphite 81 (BOPG) samples were attached to the top of a gold socket with nickel paint. The samples were cleaved with adhesive tape to provide a flat and fresh surface for imaging.

system Calibration:

The microscope was calibrated for both lateral drift and scan size by some simple experiments. For the parallel configuration, the scan size changed with changing tip length, so calibrations were required when the tip length changed significantly. Templates were used for tip cutting to reduce length variations to the order of 58 . Scan size was calibrated by imaging freshly cleaved gOPG. Fast calibration images with minimal drift distortion were acquired with the microscope 
operating in open-loop mode. A calibration image showing atomic-scale contours is shown in color plate 1. Lateral dirift of the samples varied depending on the microscope configuration and the time elapsed from assembly. Lateral drift was measured by taking a series of images at known time intervals. For the parallel configuration, initial drift rates ranges from very high to 5-10 $\mathrm{mm}$ per minute. Drifts were classified as very high when two sequential $100 \times 100 \mathrm{~nm}$ images failod to show any common features. The initial drift rates for the zoaxial configuration were about an order of magnitude lower. After 6-12 hours of operation the parallel configuration approached the lataral stability of the coaxial configuration. Low drift rates for the microscope were in the range of $1-2 \AA$ per minute.

Vertical checks of the microscope response were conducted by observing single and multiple steps on the basal plane of BOPG. Tunneling current calibration was done with dumny tunneling junctions of known resistance. The conductance signal was corrected for instrumental response by a simple nulling scheme. The high frequency perturbation used yor the bias modulation conductance measuxement produced a modest instrumental pickup. The bulk of this signal is due to capacitive coupling which is $90^{\circ}$ out-of-phase with respect to the tunneling current modulation. The detection phase angle of the lock-in amplifier was adjusted to place the capacitive coupling on the imaginary detection axis. The phase adjustment was conducted with the tip just out of turneling range. Wi.th the lock-in properly nulled, the tip was brought within tunneling range and the real component was used as the tunneling 
conductance. For a correct null, the imaginary signal was independent of the tunneling current magnitude.

\title{
Resultanand Discuseion:
}

The results presented in this section cover a range of tip types, sample types, and imaging techniques. The data were acquired over the 10-month development period of the microscope. Since the instrument was constructed in the laboratory, a considerable portion of the time for the project was spent on developing the software and hardware for the STM. The studieg reported here are a range of result used in the design modification and certification of the capabilities of the microscope. The significant contributions by the work to date are in the areas of imaging with novel tip materials, and in the application of Bimultaneour imaging and visualization.

\section{Gold surfaces and Gold With cadmium sulfide:}

\author{
Evaporated gold surfaces were one of the first sample types that \\ were tried in our microscope. These films are relatively simple to \\ prepare and have been documented elsewhere ${ }^{80}$. STM images of gold \\ evaporated films have been previously reported 74 . One major drawback of \\ gold arfaces exposed to air is their tendency to adsorb contaminants \\ (primarily hydrocarbons and sulfur compounds) from the laboratory \\ enviroment. This posed no significant problems in initial imaging \\ experiments, since the major concern was the resolution of gross
}


topographic features. For films exposed to the laboratory environment for up to a couple of days, the STM behaved in a stable manner. Apparently, thin filins of air-based contaminants do not degrade the behavior of the tunneling junction. After a few days of exposure to ambient conditions, the quality of the tunneling junction prepared with the gold film was significantly degraded. This was manifested in an unstable vertical tip position over the surface. Fluctuations of $50 \mathrm{~nm}$ to $200 \mathrm{~nm}$ were often observed under these conditions. stable junctions were (re-)attained after rinsing these surfaces with n-hexane; indicating that the destabilizing contaminanto were largely hydrocarbons soluble in n-hexane. The results reported here were obtained on films cleaned in this manner before microscope aseembly.

Illustration 39 shows a pseudo-threemdimensional representation of an image of a gold surface. This picture was constructed by one of the data display routines running on the sTM control computer and plotted on a gewlett-Packard BP7475A plotter. The first objective of the microscope was to resolve murface features of a few nanometers in size and larger. As can be seen, the rolling hill topography of evaporated gold films is well suited for testing this ability. silver crystal tips prepared by growing smal]. (approximately $100 \mu \mathrm{m}$ across) crystals from concentrated silver nitrate provided good quality images on these length-scales. These results compare reasonably with other resulte of gold films in air or condensed media. Color plate 2 shows a threedimensional surface of this data generated by a graphics supercomputer. Thene tips are suitable for the study of relatively large surface features. 
An image acquired of the same film with a platinum foil tip is shown in color plate 3. In general, the platinum tipa were better at recognizing smaller surface features in $x, y$, and $z$. The resolution of the two tip materials illustrates one of the problems with the operation of an SMM. Poor resolution STM images are observed when the effective tunneling area becomes large. This is because the image is a convolution of the contours of the tip and ample local density of states. Blunt tip ha a slowly varying lateral local denaity of states, so an image acquired with a blunt tip ahows poox lateral resolution. When the tip shape is blurred by dynamic surface processes, the tip appears blunt and resolution again decreases. The silver microcryotal tips could have blunt regions reaulting from rounding of the facet edges on a microscopjc scale. It is also possible that the tips could have resolution problems due to the low activation barrier to surface diffusion (see chapter 1). Usually, high-melting or even refractory metals or alloys are used as tip materials52,57-62. Silver crystal, or "soft", tips are useful primarily in imaging rather large features on surfaces. One unique advantage of these tips their is easy preparation and their reusability. If a bilver crystal tip is too blunt for use, the crystal at the end can be removed and regrown in a few minutes.

surface averaged, or one-dimensional, tunneling epectroscopy was used to study the band structure of gold films and gold with a thick Eila of electrodeposited cds. The tips used for the spectroscopy were platinum foil tips. The spectroscopy measurements were conducted by the application of a $3-30 \mathrm{mV}$ a.c. bias perturbation at a frequency of $3 \mathrm{kHz}$. 
The frequency of the perturbation was oignificantly faster than the response speed of the vertical control feedback loop. This ensured that the d.c. tunneling current could be held constant through feedback on the vertical position while the spectroscopic measurements occurred. Under constant tunneling current conditions, the bias perturbation produces a current response proportional to the tunneling junction a.c. conductance, (illustration 40). This is another method to measure the quantity $(\mathrm{di} / \mathrm{dV}) \mathrm{V}=\mathrm{const.}$. Many workers 60 measure this quantity by measuring current-voltage curves at constant separation and use numerical differentiation to obtain $(d i / d v) v=c o n s t . \cdot$

The tunneling junction a.c. conductance was normalized by the d.c. conductance to remove contributions to the spectra from first order area and transmission factors. This is similar to the approach of Jaklevic and Raiser ${ }^{82}$ in their measurement of Au and Pd surface-electronic structure. Their approach aintains a constant d.c. conductance while changing the applied bias through adjustments of the d.c. tunneling current set point. The normalized conductance for a bare Au surface is shown in illustration 41 . The physical quantity measured by tunneling spectroscopy is composed of Beveral components. Along with the traditional interpretation of a signal proportional to the local density of the occupied and unoccupied states, the normalized conductance signal can vary from tip-to-tip due to higher order area contributions and the normalized conductance shows a dependence on the local urface work function.

$$
\text { One major contribution to the tunneling signal is due to the }
$$
number density of states involved in the tunneling process. When both 
the sample and tip are metals, the dominant current should result from the tunneling of an electron from the negative metal to the positive metal. For a positive sample bias, electrons near the Fermi level of the tip will elastically tunnel into the sample's unoccupied states. For a negative sample bias, electrons will tunnel from the Fermi level of the sample into the tip conduction states. The highest sccupied electronic energy states in the negative material (either tip or sample) will provide the predominant tunneling signal because these states have the lowest tunneling barrier. Information regarding the number density of lower electronic energy occupied atates is clouded because of the higher tunneling barrier. As a result, only sharp changes in the number density of occupied states at a particular energy may be observed, along a varying background.

The changes in the number density of unoccupied states are significantly more apparent in the normalized conductance. This is a result of the approximately constant number of occupied states at the Fermi level. AB the energetic position of the highest energy occupied level changes, the number density of unoccupied states near this energy is probed. There is good discussion of the issue and some of the trade-offs involved in the reviews by Bamers 60 and by Griffith and Rochanski62. The major conclusion is that pronounced changes in the conductance as a function of bias are indicative of tunneling junction local density of states changes. The shape of the spectra, however, are difficult to correlate with the exact electronic state spectra of the individual materials composing the tunneling junction unless an accurate surface band structure of either the tip or sample is known a priori. 
When a semiconductor becomes part of the tunneling junction, the presence of a forbidden gap enhances the information obtained regarding the occupied states. It is possible to observe changes in the number density of occupied states below the Fermi level of the semiconductor when the Fermi level lies above the valence band edge. spectroscopic masourements of semiconductor samples with a metal probe tip reveal the positions of the conduction and valence band73,82-85. The valence band appearb prominently in the normalized conductance spectrum because of the high number of occupied states that can produce electrons that tunnel into the tip's unoccupied states. After the initial rise in conductance due to the onset of tunneling from the valence band, the most pronounced features would arise from changes in the density of unoccupied states in the tip, all other things being equal.

Illustration 42 shows the normalized conductance signal for a thin filin of cds on a gold surface. The cds film was deposited electrochemically by reducing thiosulfate in the presence of $\mathrm{cd}^{2+}$. The presence of small "isolated" Cds grains on the surface was verified by overtone RAMAN 86 Bpectroscopy. The vertical stability of the tunneling junction with this film was very poor. No stable, reproducible images of the surface were recorded. However, the ourface-averaged conductance Bignals were Belf-consistent and reproducible. Surface-averaged conductance was measured by scanning the tip over the surface with the conductance detection channel set at a time constant of 3 seconds. When comparing the spectra, illustrations 41 and 42 , there are major differences which can be attributed to the addition of a cds filin and band structure with a forbidden gap to the system. The band gap of bulk 
Cds is $2.42 \mathrm{eV}$, cds particles of emall size display wider band gaps 87,88. The approximately 3 eV gap between the regions labeled as valence and conduction bands is sensible for this ourface deposit. These experinents with gold evaporated films allowed simple checks of drift and tip preparation schemes. The work in one-dimensional spectroscopy laid the foundation for instrumental design for the spatially resolved spectroscopic measurements. The results here are consistent with the results obtained by previous work on gold surfaces and other semiconductor surfaces. Ruk and silverman 90 report similar spectra to our result for the bare gold ourface, both our and their results differ in appearance on the positive sample side from the work by Kaiser and Jaklevic 80 . However, Kaiser and Jaklevic point out that the feature they observe is probably due to a surface state, so the inconsistency may be explained by cample preparation differences. Although direct measurements of CdS band structure have not been reported, work by everal groups on semiconductor substrates has observed iasd-gap type features $73,82-85$.

\section{Dlatinum surfaces:}

Platinum surfaces were imaged in air with platinum foil or tungsten based tips. The gross surface structure and the presence of local work function changes on surface "facets" were studied. Simultaneous imaging techniques produced new information regarding the properties of polycrystalline platinum surfaces exposed to air. A high resolution topograph of a typical platinum surface is shown in color 
plate 4. Steps, terraces, and a canyon provide a typical microscopic view of the platinum surface. However, the information about a realistic surface which can be obtained from this type of data, is limited. For example, the presence of airborne contaminants is not revealed. It is possible that this metal surface is free of surface contamination, but not likely. This is a major drawback of experiments that measure only topographic information using the STM. Topographic data obtained on typical surfaces does not usually contain sufficient information to determine the molecular or chemical identities of features on the surface.

This type of drawback exists for many commercially available sTM systems. This was one of motivations for developing an STM/s instrument within our lab. The instrument designed and constructed as part of this research is tailored for experiments related to discerning certain types of chemical differences on realistic surfaces. Our approach uses the simultaneous measurement of multiple tunnel-junction observables as a means of performing this type of recognition. Color plate 5 shows two renderings of the ame topographic data which illustrates this idea. The top picture is a three-dimensional representation of the topography of a platinum aurface, imaged in air; while the bottom picture is a combination of this topographic data with color-coded data from the a.c. conductance at each point on the surface. The bottom picture is obtained by texture mapping the conductance image onto the threedimensional topography. The additional information regarding relative conductance differences between several surface regions is then easy to visualize. 
Theoretical expressions (like equation 24) for the tunneling conductance signal under conditions of constant current reveal that two major components have contributions which can cause the relative changes in conductance shown in color plate 5 . One possibility is that the local density of electronic states at this sample bias is different from one surface plane to another (the first term in the numerator in equation 24). This is the traditional interpretation of sTs images, namely that spatially vaxying conductance signals are attributed to changes in the local density of electronic states. Another possible explanation of the changes in relative conductance over the surface is a localized change in surface dipole, affecting local tunneling barrier heights (as mentioned earlier, $A\left(V_{B}\right) / B\left(V_{B}\right)$ in equation 24 is related to the local barrier). Under appropriate normalization for tunnel junction d.c. conductance, the small-signal conductance is linearly dependent on local barrier changes. The dependence of the normalized a.c. conductance on the number density of states is significantly less than linear, as evidenced by the small changes in the quantity when studying semiconducting samples. In the presence of local barrier changes, therefore, Iocal changes in the number density of states will be difficult to detect because of their small contribution to normalized conductance signals.

In light of this, we attribute the relative variation in conductance of 308 in color plate 5 to local work function changes. Differentiating equation 23 and employing a trapezoidal barrier, this relative conductance change correlates to a variation of about 1.2 ev in the tunneling barrier. A platinum surface exposed to airborne 
contaminants could readily acquire surface species capable of producing surface dipole changes of this magnitude91,92. Adsorbates are indicated in this case since the variation in work function of clean platinum surface planes $(<111\rangle$ to $\langle 100\rangle)$ are about a factor of 5 to 10 too small. surface work function changes observed on platinum surfaces exposed to aromatic adsorbates are on the same order as the changes observed here 92 .

More carefully controlled experimental conditions are required to establish the chemical nature of the adsorbate and verify these conclusions. However, this application of simultaneous imaging to obtain surface chemical information shows promise. This example highlights the utility of multiple imaging techniques at a single junction bias. Surface conductance changes can be directly used to differentiate "bumps" and surface features on the basis of local work function changes. Promising work in our laboratory is continuing in the area of recognition of semiconductor clusters on rough metal surfaces.

\section{Bighly Oriented Pyrelytic Graphite Samples:}

The imaging described in the previous section was performed at one bias. Under those conditions, the conductance signal is a useful diagnostic; however, its utility as a "spectroscopy" tool is only realized when the simultaneous inages are acquired at a range of biases. Data on a highly oriented pyrolytic graphite (HOPG) surface exposed to air were acquired over the sample bias range of -0.5 to $0.4 \mathrm{eV}$. These images were acquired over the course of about 30 minutss and captured a 
region of the surface with small steps separated by terraces. Color plate 6 shows one simultaneous image with topography and texture-mapped conductance at one bias.

Stepped regions like this are uncommon on freshly cleaved HOPG surfaces. Usually, approach of the tip and imaging revealed perfectly flat terraces from $500 \mathrm{~nm}$ to $1.0 \mu \mathrm{m}$ across. Massive defects, see color plate 7, and steps as in color plate 6 were found during active searches of the surface for such features. These surface features turned up over the course of systematic searches for surface defects that lasted for a few months of microscope operation and testing. The defect region in color plate 7 appears to be a result of a twist boundary. There are two major domains to the right and left. At their intersection, step and terrace lines slew off in a range of directions, of which only a fraction are consistent with the hexagonal oymetry of the surface. Quantitative spectroscopic information for the simple step and terrace system in color plate 6 can be obtained by analysis of the relative conductance at each surface position. This is accomplished by an analysis program that takes the two-dimensional image data and extracts the scan line values. This program can use a single scan line or average over several lines to increase the gignal to noise ratio. A plot of the vertical and conductance signal for one bias is shown in illustration 43 . The vertical scale, primary y axis, is significantly magnified with respect to the surface position on the $x$ axis. This plot is repregentative of the data; there are terrace regions with slight planar misorientation and steps between the terraces. 
The conductance signal at the step is significancly higher than the terrace conductance signal. To convert the set of images into specific spectroscopic information for particular aurface features, the following data processing was performed. For each image, data like that shown in iliustration 43 were extracted. Then, the conductance at the step region and the conductance over the terrace region were normalized by the d.c. conductance. Finally, the data are compared for the terxaces and the steps; illustration 44 displays the normalized conductance data obtained by analyzing all the images. The "v" shape of the two sets of values indicates that the nemalized conductance has a roughly linear dependance on sample bias over this range of bias. This Iinear change in normalized conductance indicates that the major difference between the step and the terrace lies in the local work function. The ratio between local atep work function and terrace work function is obtained by dividing the slope of the step data by the slope of terrace data. Assuming that bulk surface values for the work function are close to the terrace value, the local work function at the step is lower by $1 \pm 0.5 \mathrm{~V}$ (958 confidence values from linear fits to the data).

The sign of the local work function change agrees with theoretical models of electron density at a step in a jellium metal93-95. Near a step in the surface, electronic dezsity spills outward parallel to the plane of the surface, creating a net negative charge at the base of the step and a net positive charge at the top of the step 96 . From illustration 43 , we see the spatial extent of the lower local work function is on the order of a few nanometers, which indicates a modestly 
delocalized perturbation to the surface dipole. The component of this charge separation at the step that lies normal to the surface is aligned against the jellium surface dipole, reducing the work function locally around the step 95 . However, the calculations by Thompson and Buntington 96 for steps on a sodium surface suggeat a locally induced potential change around $0.1 \mathrm{eV}$, about an order of magnitude less than observed here. Besocke et al.97 present measurements of work functions for stepped metal surfaces of tungsten, silver and platinum. Their observations are consistent in magnitude with the calculation by Thompson and Buntington. They do point out that the variation observed in step induced work function varies oignificantly with the metal. We propose that the large inter-plane spacing is producing higher than expected work function changes at the steps. Since graphite is a rather unique metal, this could still be a reasonable explanation.

Another possible explanation is that the jellium model is not appropriate to describe this experimental result. In this case, we would need to apply a theory of a surface and ateps from a more molecular, localized standpoint. However, the behavior over energy (illustration 44) tends to rule out energetically localized gtztes. A final possible explanation is the presence of chemical inhomogeneity at the step, like the platinum surface. This has been eliminated as a strong contender because evidence gathered while acquiring all of the data in illustration 44 suggests that sheets were stripped off the surface during the course of the experiment. This effectively presented a fresh, uncontaminated surface with steps at the start of oeveral of the scans. Additionally, other experiments in our laboratory have found 
that stepped BOPG surfaces are quite resistant to chemical attack, even oxidation 98 .

The differences between the terrace and step oignal in illustration 44 is quite striking. There is a more subtle difference between the relative conductance signals between the terraces themselves. The difference is guite obvious in color plate 6 , where one terrace has a bluish cast while the other is yellowish. The bluish color is indicative of low relative conductance and a relatively higher work function or lower number density of surface electronic states. For graphite, a material where the interactions between the sheets are relatively small, stacking faults may be common. It is quite probable that a stacking fault producing a sequence of ...abcaba would produce a surface that was slightly different electronically than an unfaulted surface layer ...abcabc. This hypothesis is supported by the significant change demonstrated in graphite band structure calculations 99 when a neighboring layer is allowed to interact with a graphite sheet. The experimental percentage difference between the two terrace types in the conductance is approximately 58. These two terrace types were observed multiple times during the systematic search of the BOPG samples for defects. Only two relative conductance levels were observed in these studies, supporting the faulted ve unfaulted surface model. 
In this chapter, the surfaces and Iosults have spanned tho range from reproducing other work to the application of simultaneous imaging techniques to platinum and HOPG. The availability of oilver microcrystal tips opens possibilities for unique quality control by insitu tip modification via crystal growth. simultaneous imaging of platinum surfaces has provided a glimpse at eurface region differences which are nomirally not apparent to STM. spectroscopic Bimultaneous imaging techniques have hown local electronic changes at steps on HOPG. Finally, terrace stacking faults have been observed, again detection of subtle surface differences not apparent to traditional scanning tunneling microscopy. 


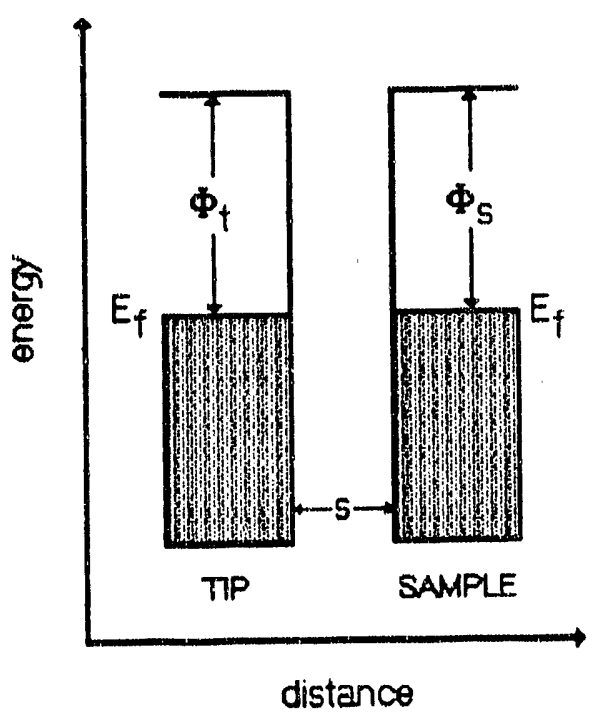

a)

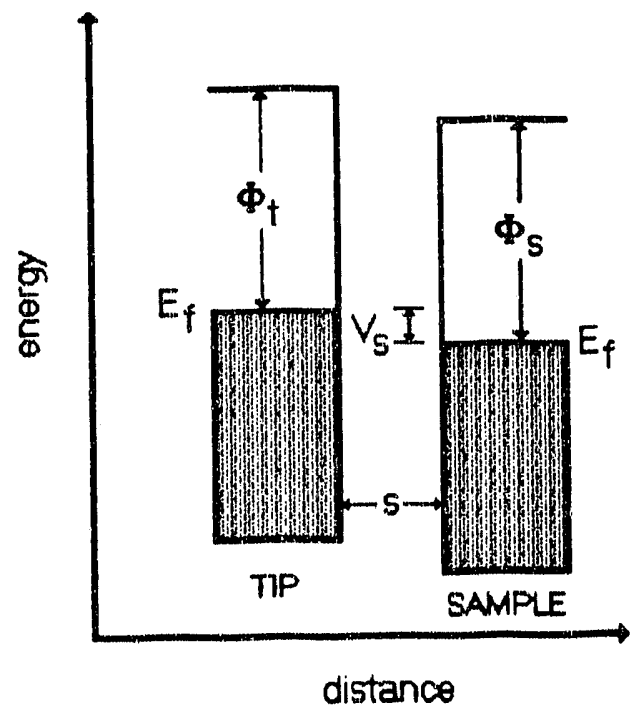

b)

illustration 34: a) schematic of band structure of a homogeneous junction with no applied bias. Shaded areas denote occupied stater; for metals, the overlap of the conduction band with the valence band results in delocalized electronic states near the Fermi energy, $\mathbb{z}_{f}$. For small B, overlap of delocalized electronic states between the materials will allow electron tunneling from tip to sample or vice verba. b) If the sample is at a positive bias $\left(V_{B}\right)$ with respect to the tip the net electric field will drive net flow of electrong from tip to sample. since the tunneling probability is roughly exponentially dependant on the height of the potential barrier, the highest energy electrons (near $\mathbf{E}_{f}$ of the ti.p) will be the major contributors to the net flow of electrons in this case. This results in a roughly local probe of the unoccupied states in the sample around the energy $E_{f(B)}+e V_{B}$. 


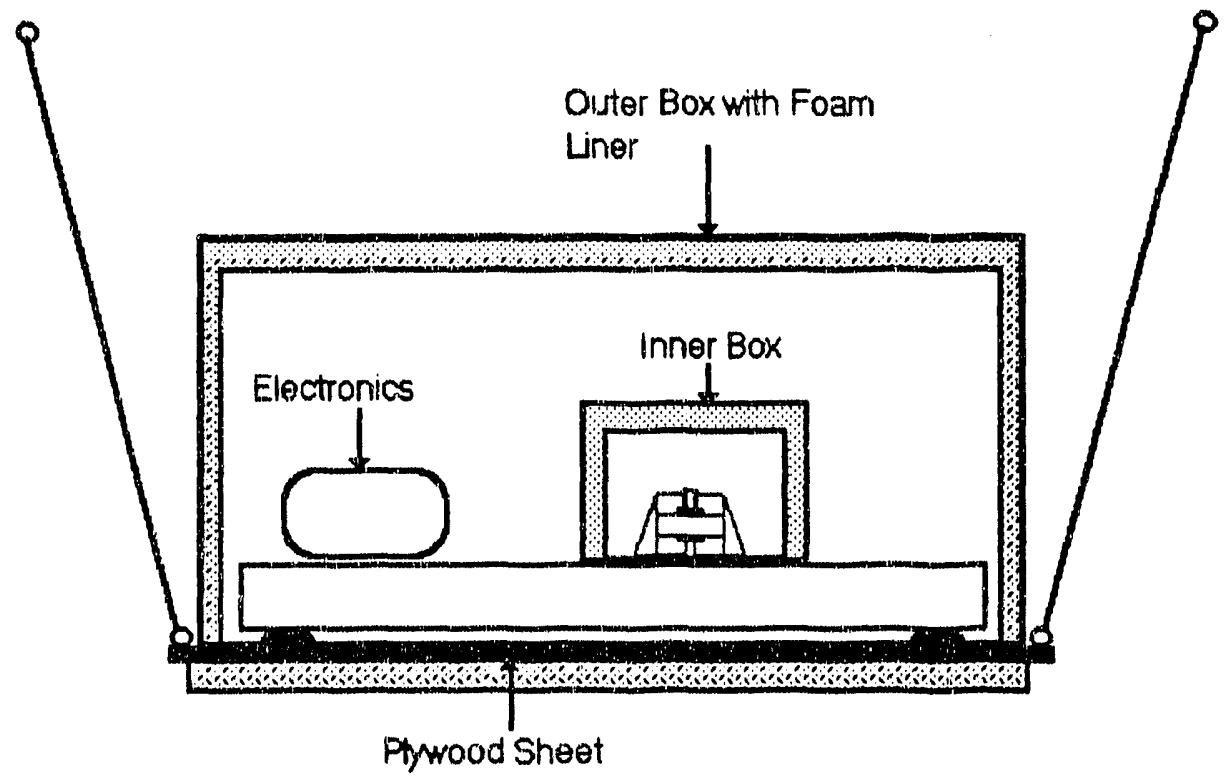

illustration 35: physical mount for microscope apparatus. The plywood sheet is suspended by elastic cords. An acoustic/thermal shielding box rests on the plywood sheet. Invide the box, a Newport $3^{\circ} \times 2^{\prime}$ optical table sits on 4 ar-filled bladders. The microscope electronice and local acoustic shielding hox are mounted to the top of the optical table. 


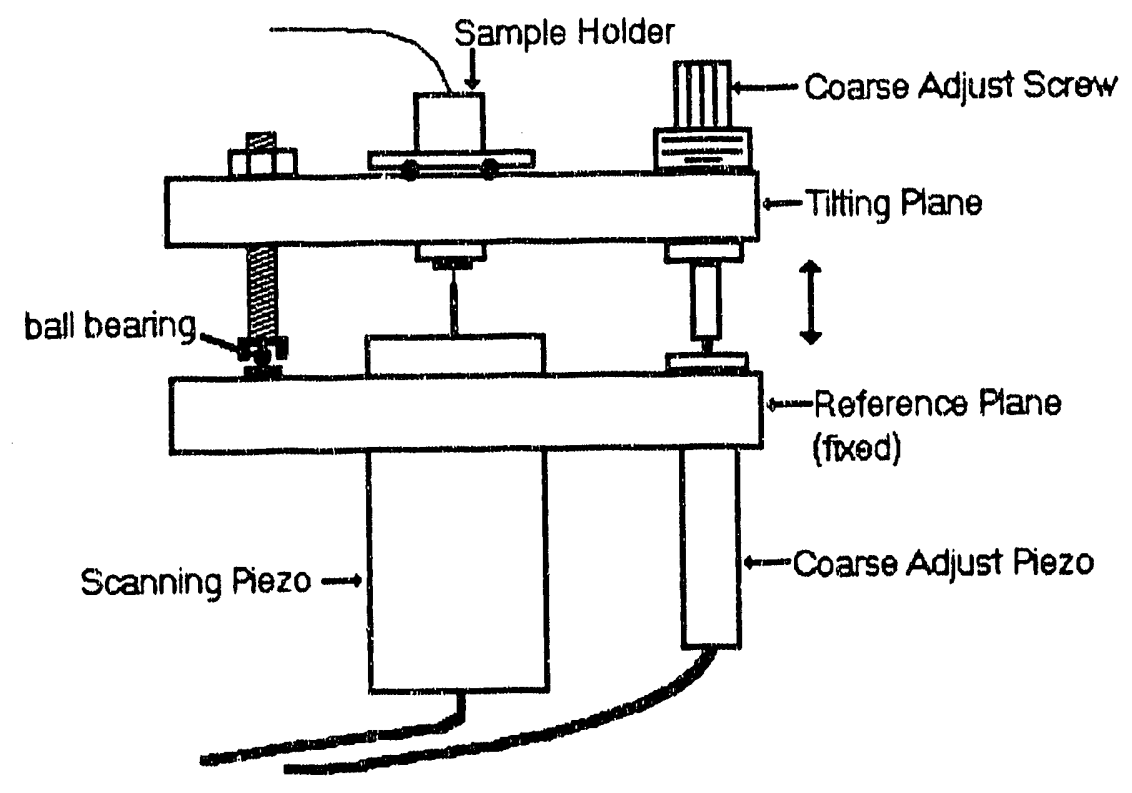

illuatration 36: Parallel configuration in a simplified side view. In all, 6 contact points by ball bearings were used to position the tilting plane. Two of the three for vertical control are shown here. There are two bolts used for gross separation control. Once locked, these bolts were not moved. Coarse approach was accomplished by shortening an 80 thread-per-inch screw, producing rotation of the tilt plane at the other ball bearing point contacts. The wires associated with the piezoelectric elements and the tip were rigidly mounted to the optical table. The sample wire is a light-weight wire to prevent transmisaion of residual stress and vibrations. 


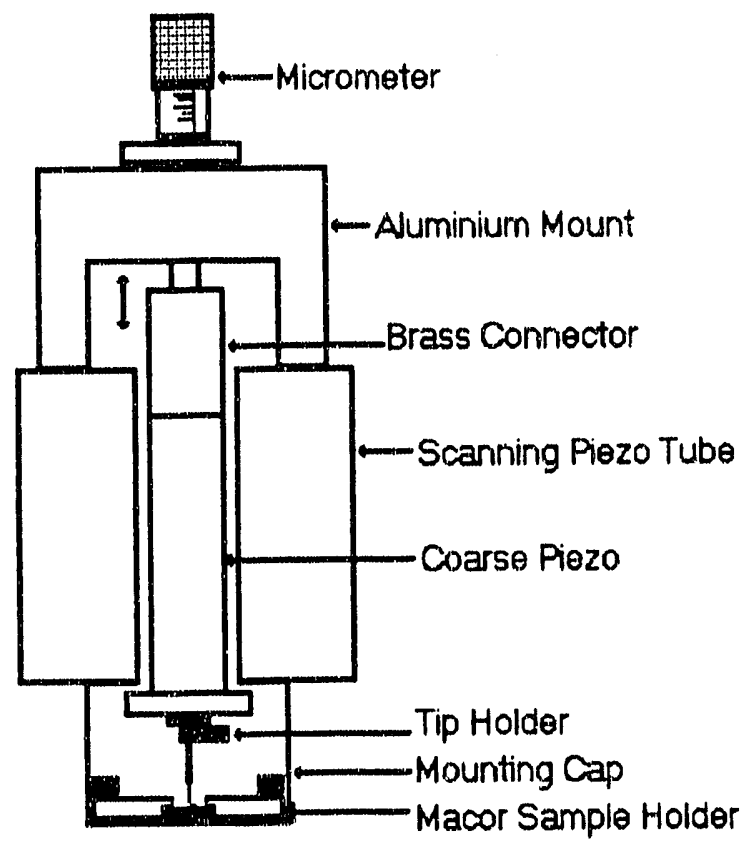

illustration 37: Cross-section of coaxial configuration. The Bcanning piezo element (Burleigh $\mathrm{PZ}-81$ ) has a central bore large enough to accomodate the coarse PZT pusher. A brass fitting on the top of the coarse piezo element is connected to a rotationless micrometer spindle. Coarse mechanical approach is accomplished by the micrometer which moves the coarse piezo within the scanning element. The tip holder is affixed to the end of the coarbe PZT with a magnet. Samples may be replaced from the bottom aide of the MACOR holder without removing the Bample cap. 


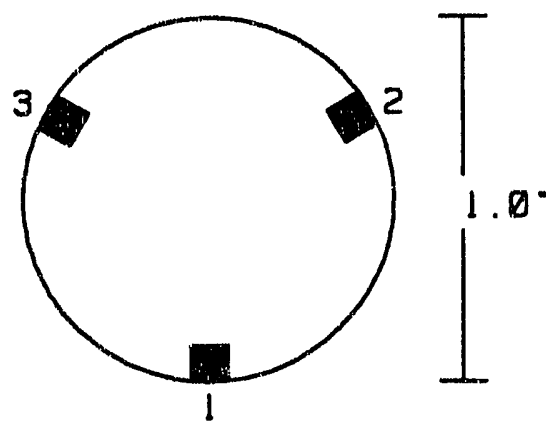

TOP VIEV
Z TRANSLATIION: $\quad$ X TRANSLATION:
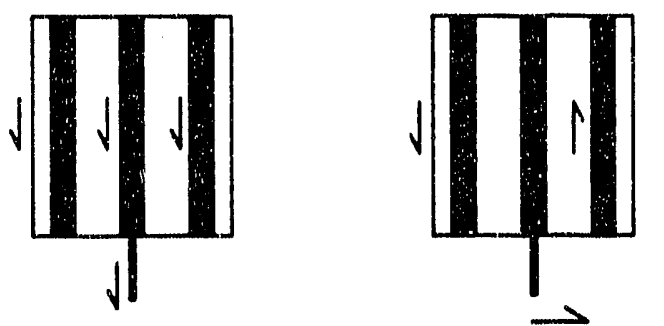

SIDE VIEVS

illustration 38: Configuration of piezoelectric elements in the Burleigh scanning PZT. The side views show the symetric mode that produces $z$ translation of the tip and the asymetric mode that produces $x$ translation of the tip. The transformations were identical to the orthogonal modes of the appropriate symmetry point group, $\mathrm{C}_{2 \mathrm{~V}}$. 


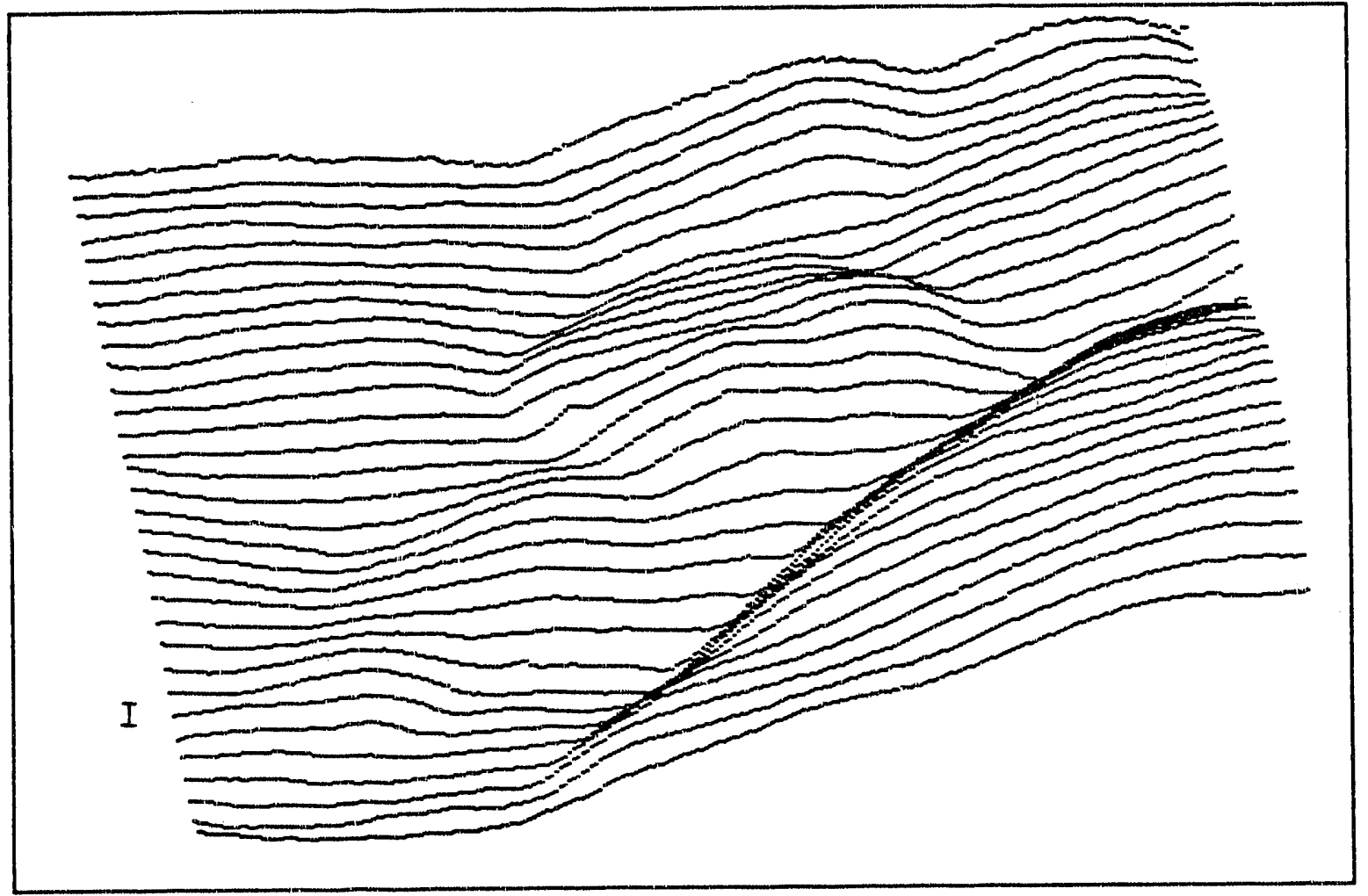

illustration 39: Line representation of a gold film as viewed by a silver microcrystal tip. The imaging area is $100 \mathrm{~nm} \times 100 \mathrm{~nm}$. The scale bar to the left is $5 \mathrm{~nm}$ high. Every fourth acan line is shown here to reduce clutter. On a monitor screen, every other scan line produces an acceptable picture. Scan parameters for this image; scan time 2 minutes, sample bias $0.25 \mathrm{~V}$, tunneling current $0.2 \mathrm{nA}$, peak-tovalley height $80 \mathrm{~nm}$. Two local average passes were run on the data before generating the figure. 


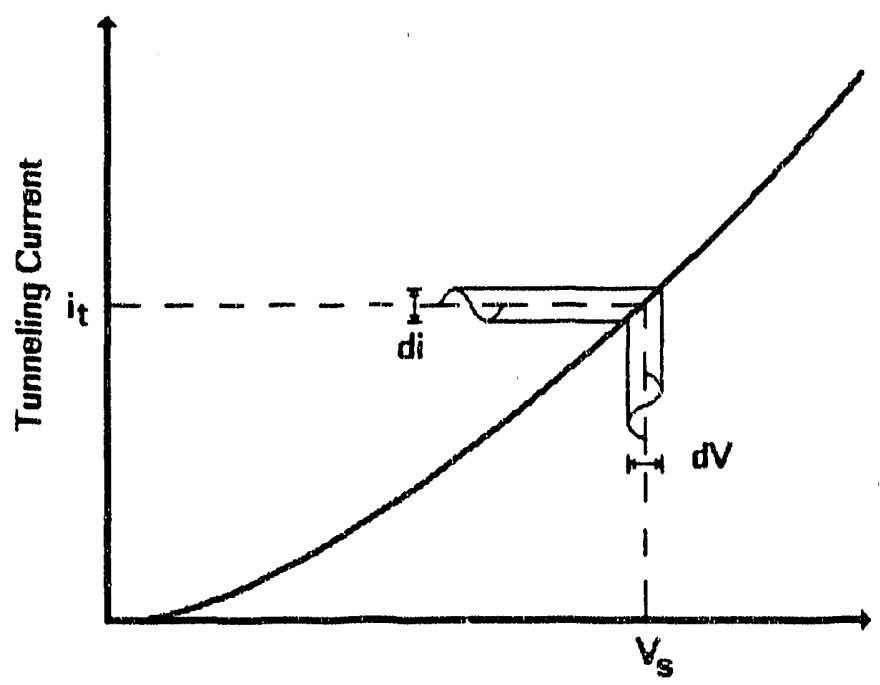

Sample Bias

illustration 40: sketch of conductance measurements. A constant bias is applied, $v_{B}$; with active feedback, $i_{t}$, is maintained. A small bias perturbation dV is applied and the response di is measured by a lock-in amplifier. As long as $d V$ is small compared to $v_{8}$ the quantity $(\mathrm{di} / \mathrm{dV})_{V=v_{B}}$ is obtained. Typically, we use $d V=3-30 \mathrm{mV}$ and $v_{B}=0.1$ -2.2 . 
Pt/air/Au Junction Conductance vs. Bias

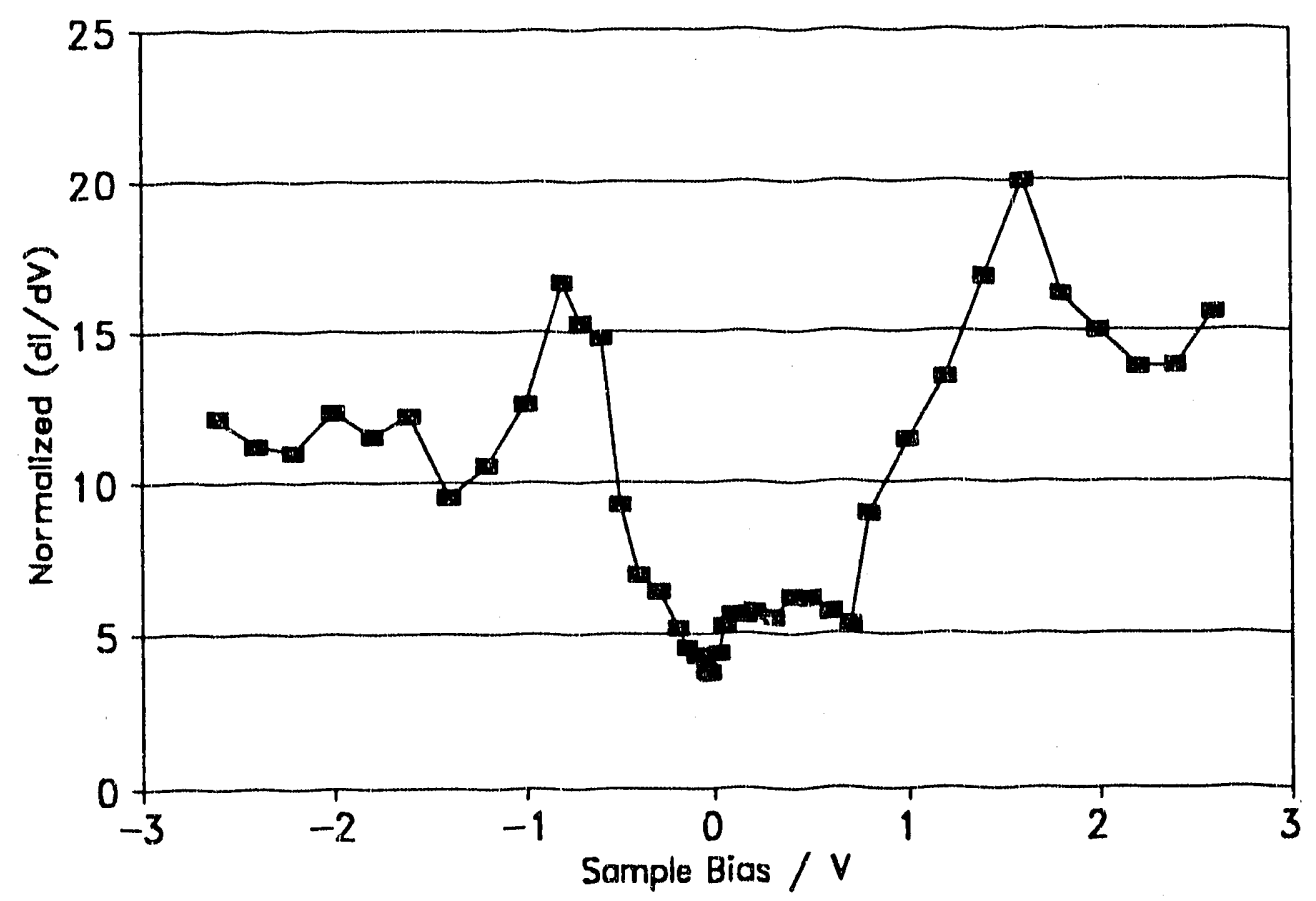

illustration 41: Tunneling junction conductance spectrum of a bare gold surface acquired at room temperature. The normalization is obtained by multiplying the lock-in response by the absolute value of the bias and dividing by the tunneling current. 
Pt/air/CdS/Au Junction Normalized

Conductance vs. Bias

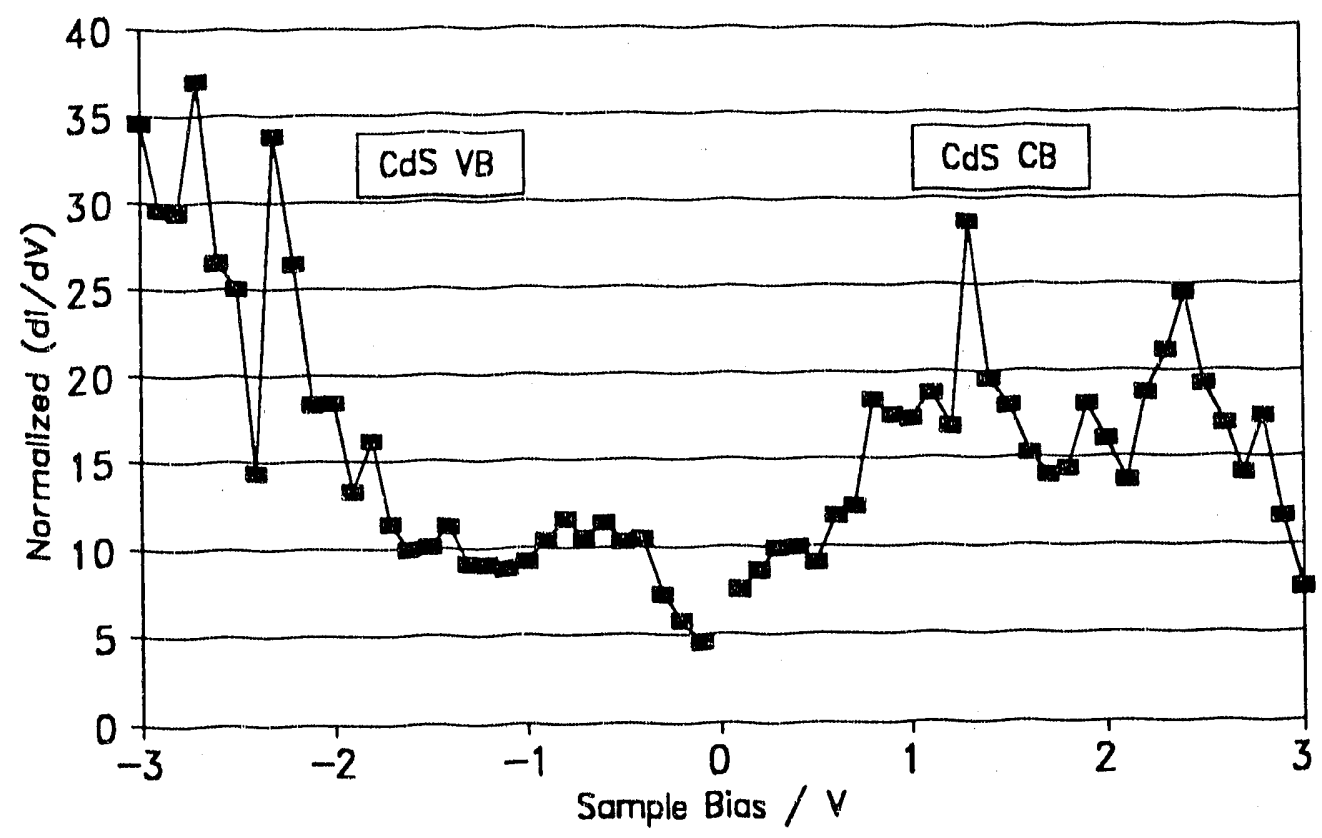

illustration 42: Tunneling junction spectrum of gold with a thin film of cds. Normalization produces the same units as in illustration 41. Comparing to illustration 41 , the new features are the wider, flatter band at positive bias and the sharp signal increase at sample bias $-2.3 \mathrm{~V}$. Both features are consistent with the addition of material with band atructure similar to cadmium eulfide. The very negative pinning of the cds valence band (VB) with respect to the Fermi level of gold has also been observed elsewhere ${ }^{89}$. 


\section{Linescans for Topography and Conductance}

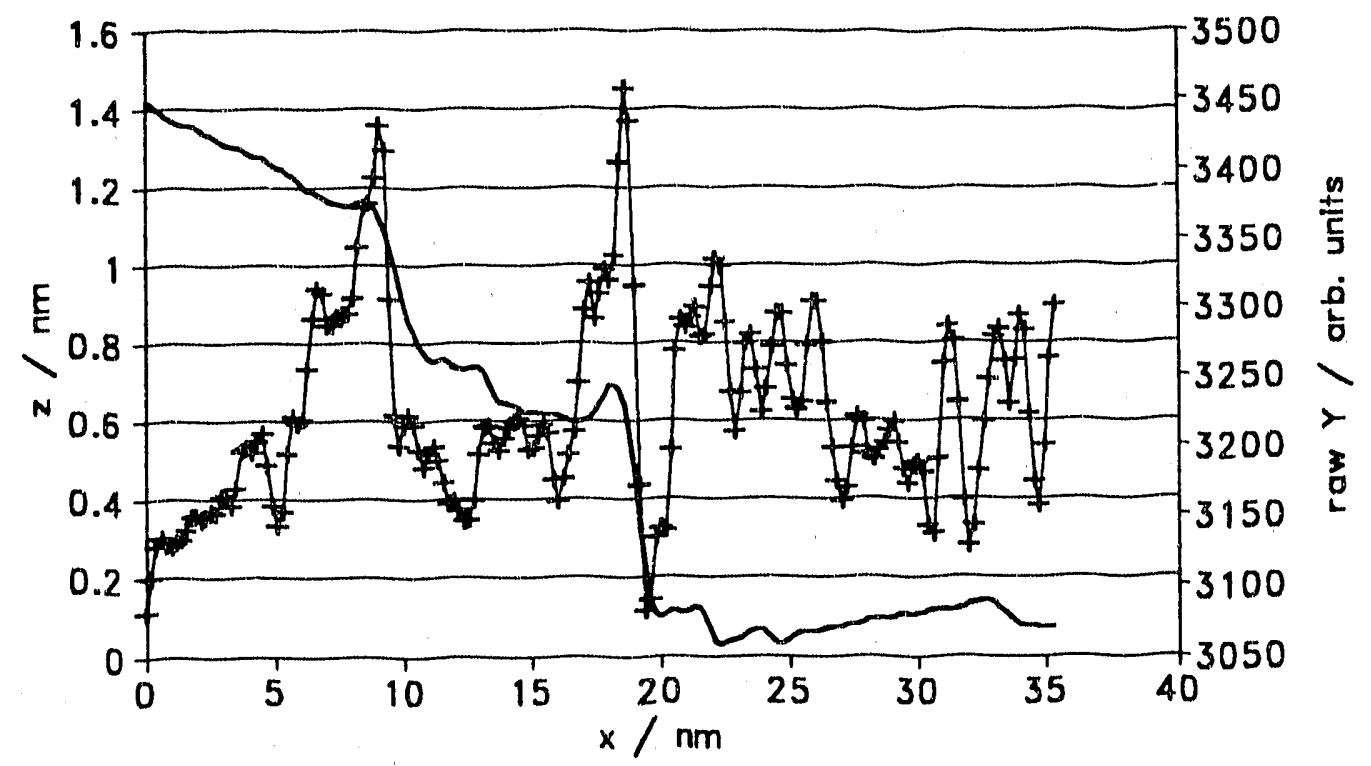

- topography + conductance

illustration 43: Topography and conductance aignals averaged over 20 lines for a step and terrace region of BOPG. This data is extracted from a simultaneous image acquired at sample bias $-0.1 \mathrm{~V}$ and tunneling current $1 \mathrm{nA}$. The topography data is exaggerated by about 15 times with respect to the horizontal (distance) axis. One single-layer step and one two-layer step is visible; the two-layer step appears to separate two different terrace types. The actual misorientation angle between the terraces at left and at right is about $2^{\circ}$. The differences between step and terrace conductance are apparent as well as the conductance difference. 
Normalized Conductance vs. Bias

W/WOx/C/air/C junction 25 degrees C.

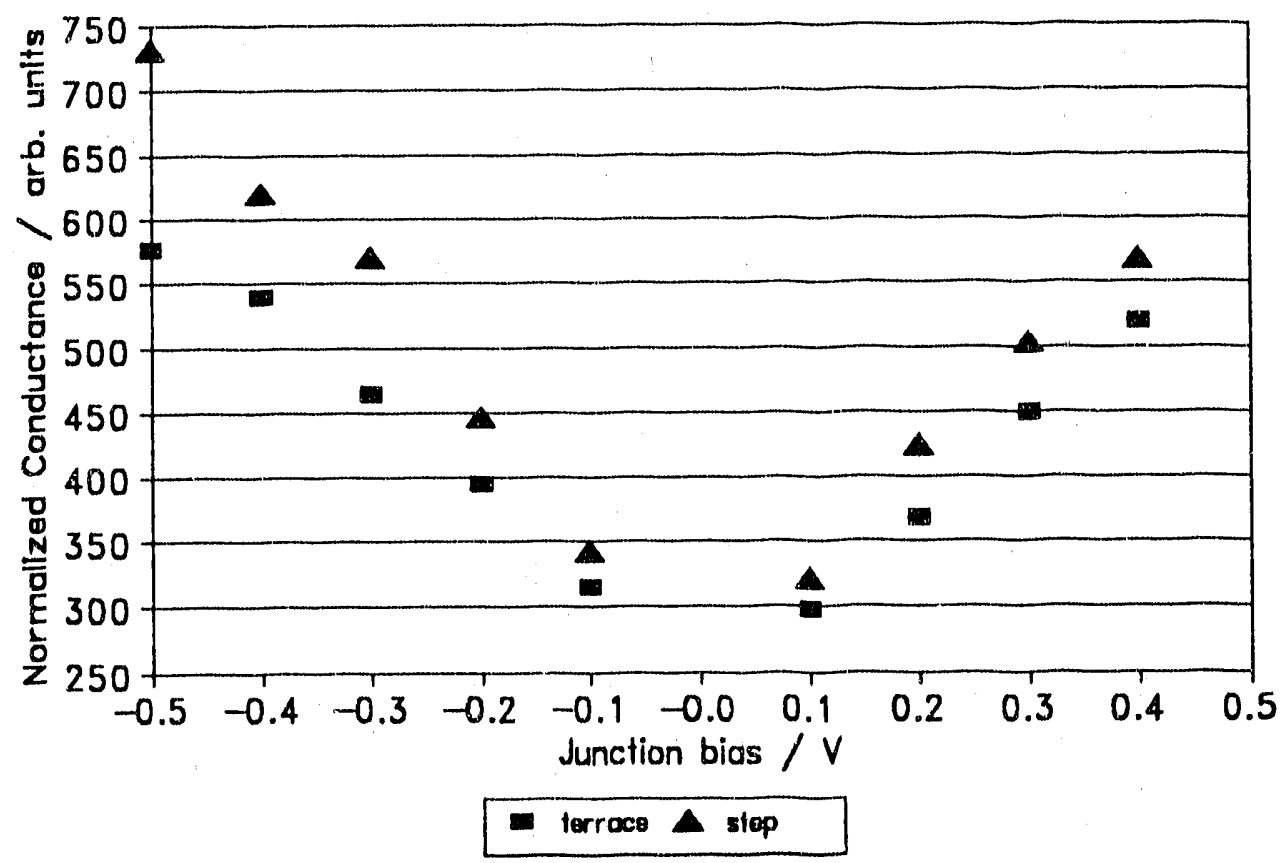

illustration 44: Voltage behavior of normalized conductance over a omall bias window. The almost linear increase in normalized conductance with bias is indicative of constant barrier height and relatively amall variation in local electronic atate density. Steps of 1 to 2 HOPG Cspacings are compared to terraces. The majority of the difference appears to result from local work function differenses. The minor, but observable difference in slopes for the terrace and the step data are consibtent with a $1 \pm 0.5 \mathrm{~V}$ lower work function at the steps. 
Color Plate 1: Open-loop mode calibration image of highly oriented pyrolytic graphite (HOPG) acquired with a wox tip. The observed corrugations are a result of current variation during the scan. Due to drift during image acquisition, the corrugations are distorted olightly from their ideal hexagonal shape. This is not readily apparent from the image here; however, it places significant bars on the absolute calibration of the image size, $2.6 \mathrm{~nm} \times 2.6 \mathrm{~nm}$ ( $\pm 3 \mathrm{~nm}$ per side). By using the tip length, geometry of tip holder and magnitude of signal to the scan element, the image is calculated to be $2.5 \mathrm{~nm} \times 2.5 \mathrm{~nm}$. The data has a grayscale image of the current texture-mapped onto the threedimensional physical map of the current. The data has been downsized by a factor of 2 , the geometric data is illuminated with one light and Gouraud shading is used in the rendering. 


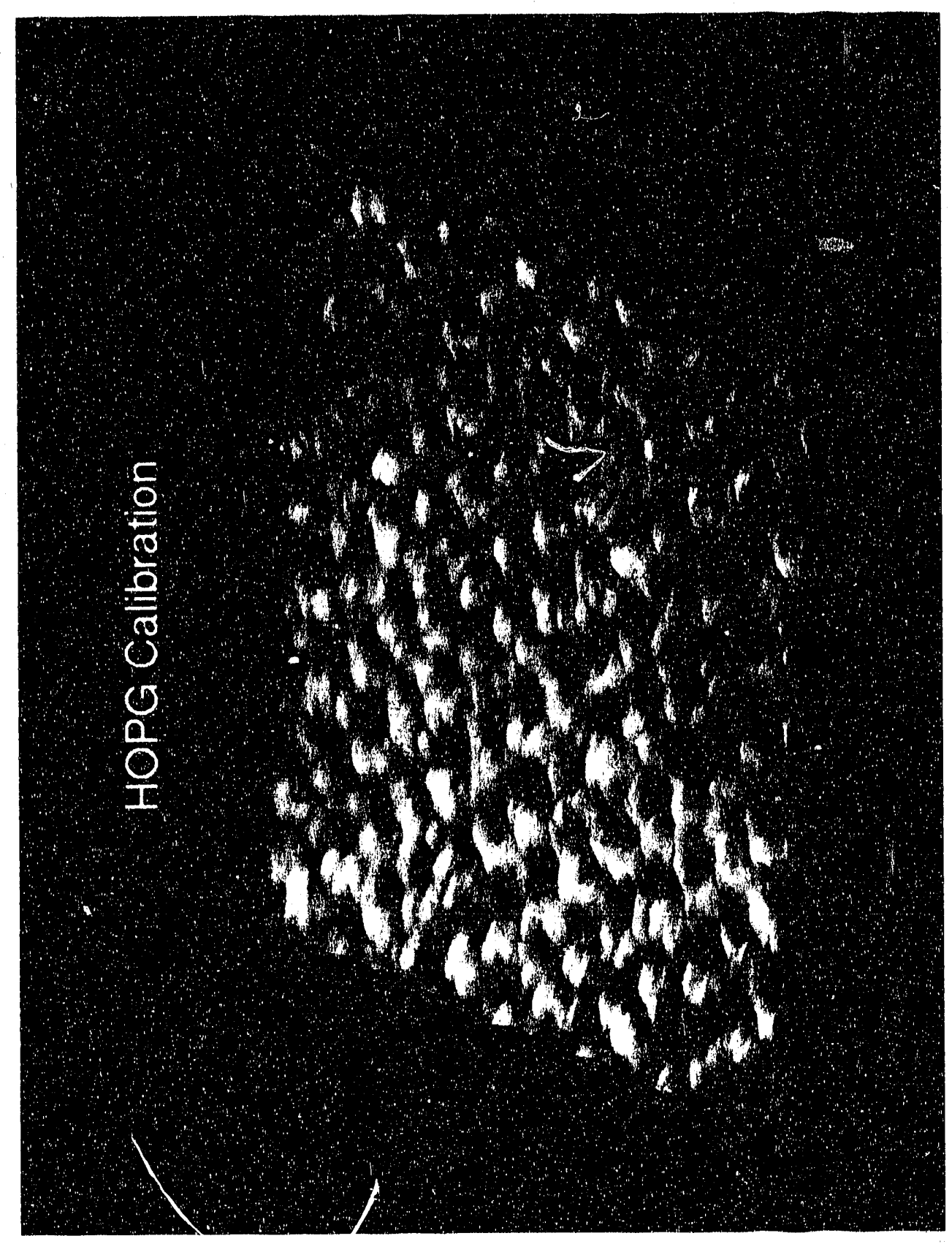




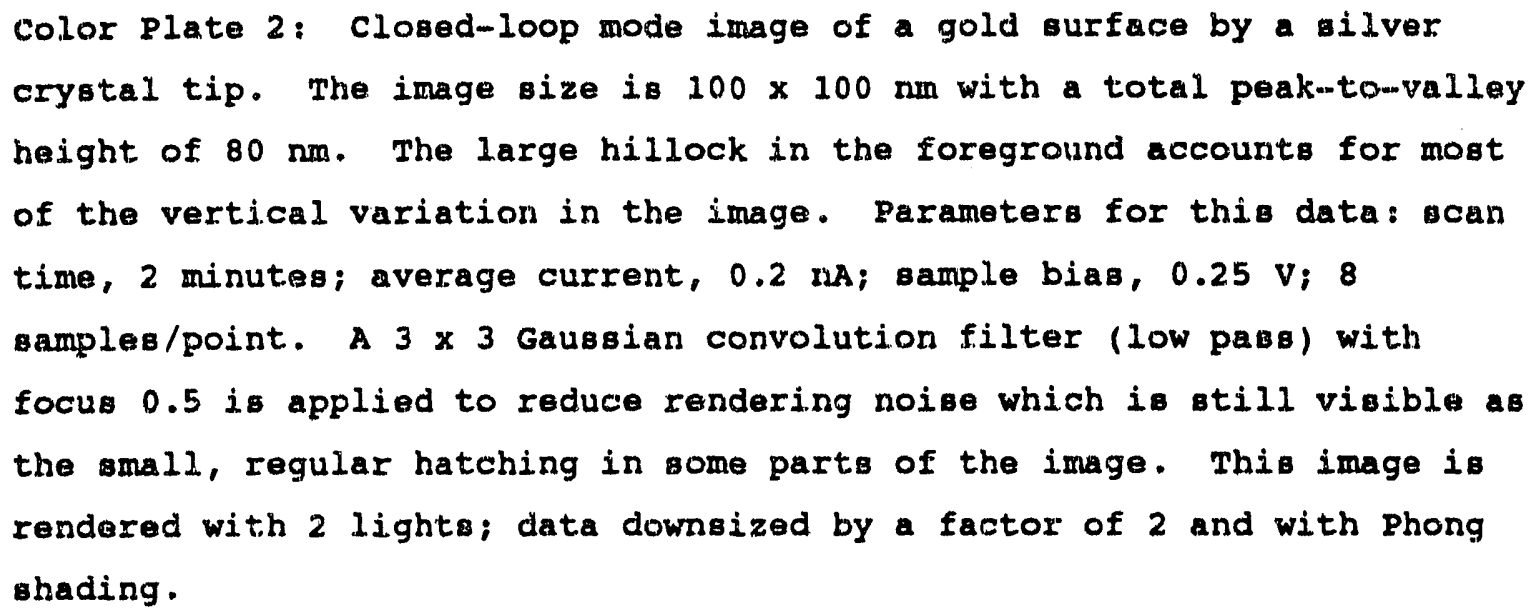




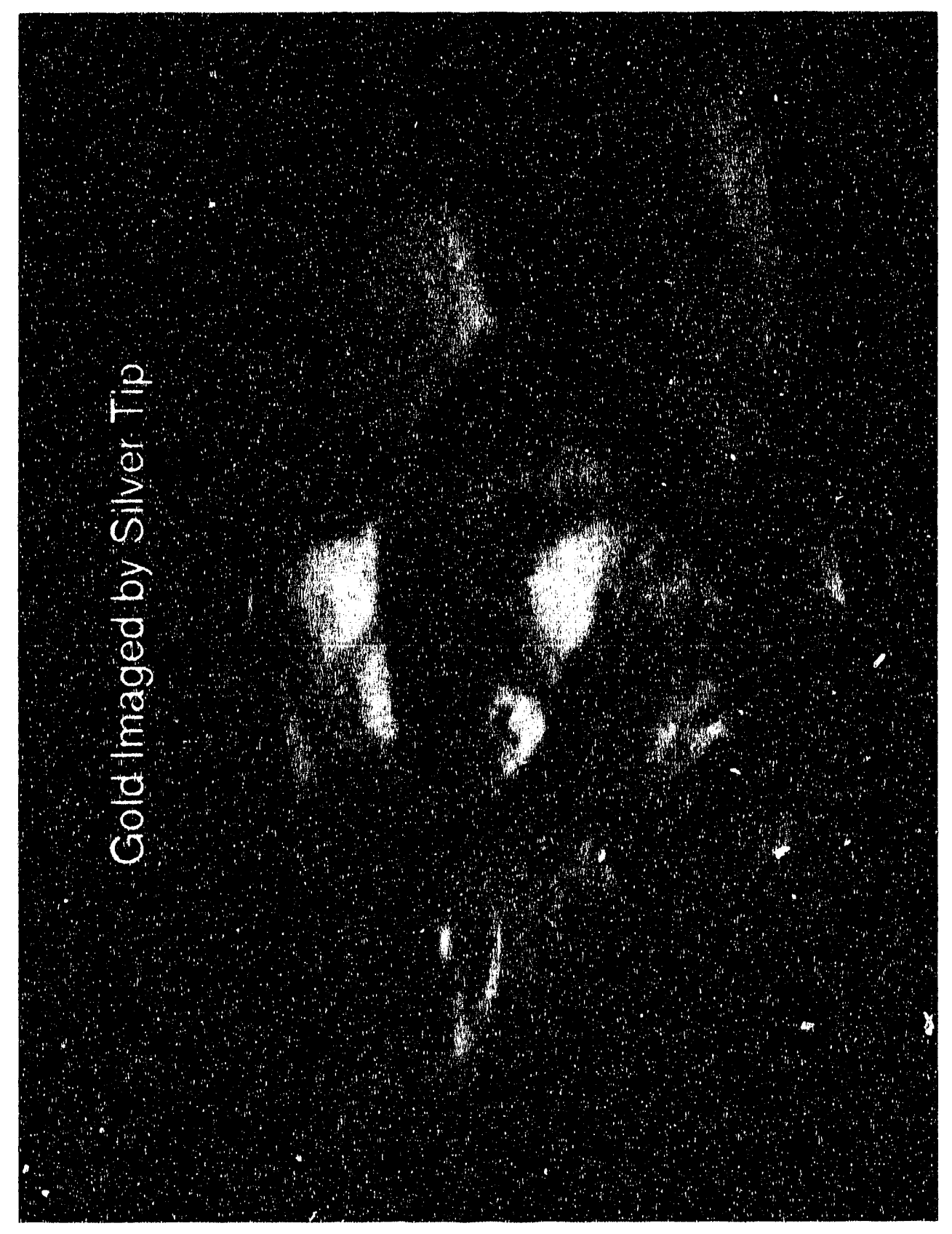


Color Plate 3: Closed-loop mode image of a gold surface acquired with a platinum tip. The image size is 100 x $100 \mathrm{~nm}$ with a total peak-tovalley height of $5 \mathrm{~nm}$. The vertical enhancement between this data and the previous image (color plate 2) is 6.4 times. Parameters for this data: scan time, 30 seconds; average current, $1.0 \mathrm{nA}$; sample bias, -0.25 V: 4 samples/point. A 3 × 3 Gaussian convolution filter (low pass) with focus 0.5 is applied to reduce rendering noise. This image is rendered with 2 lights; data downsized by factor of 2 and with Phong shading. 


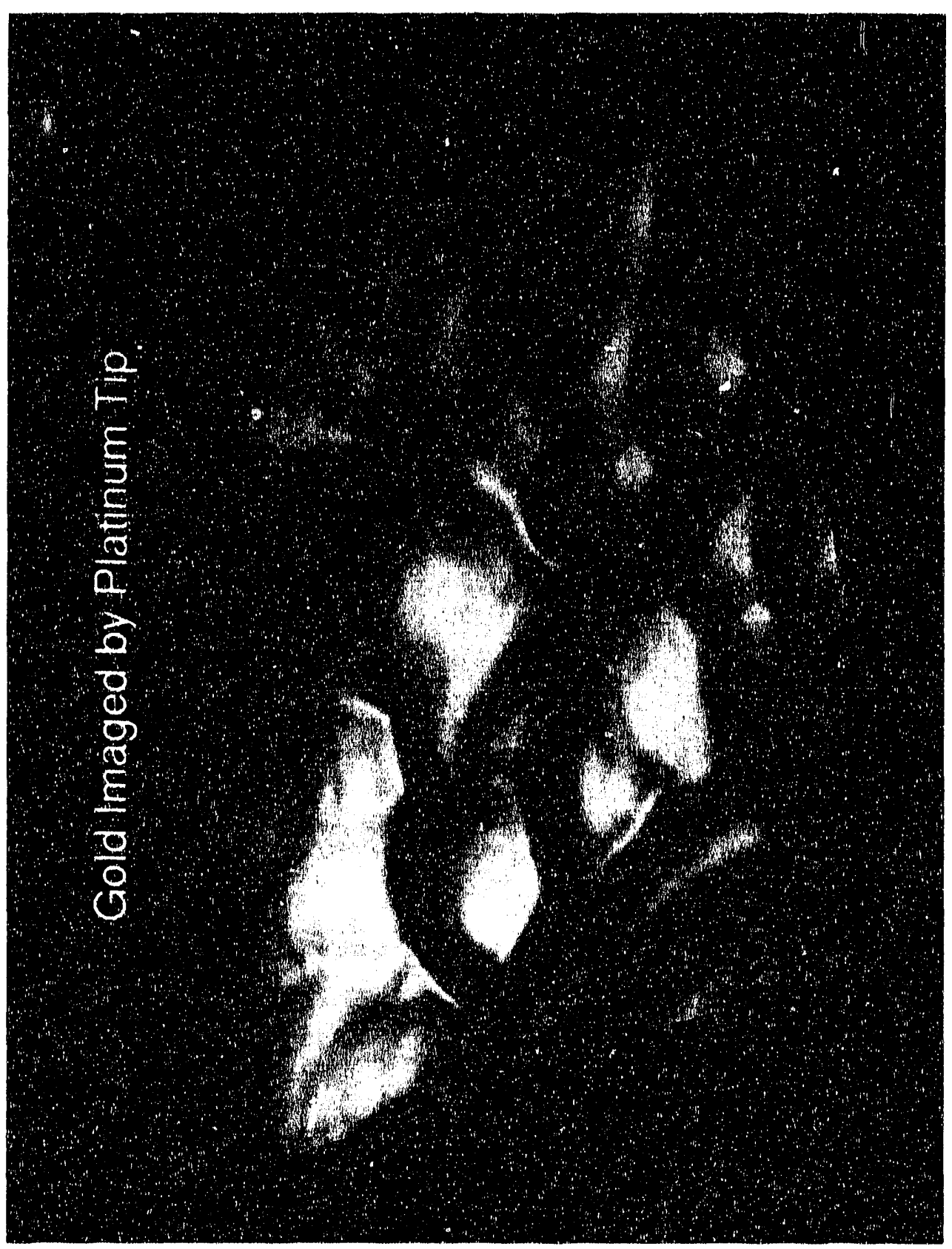


Color Plate 4: Closed-loop mode image of a platinum surface acquired by a platinum tip. The image size is $120 \times 120 \mathrm{~nm}$ with a total peak-tovalley height of $14 \mathrm{~mm}$. Parameters for this data: scan tima, 1 minute; average current, $1.0 \mathrm{nA}$; sample bias, $0.25 \mathrm{~V}$; 8 samples/point. A $3 \times 3$ Gausian convolution filtar (low pass) with focus 1.0 is applied to reduce rendering noise. This image is rendered with 2 lights; data downsized by a factor of 2 and with Phong shading. 


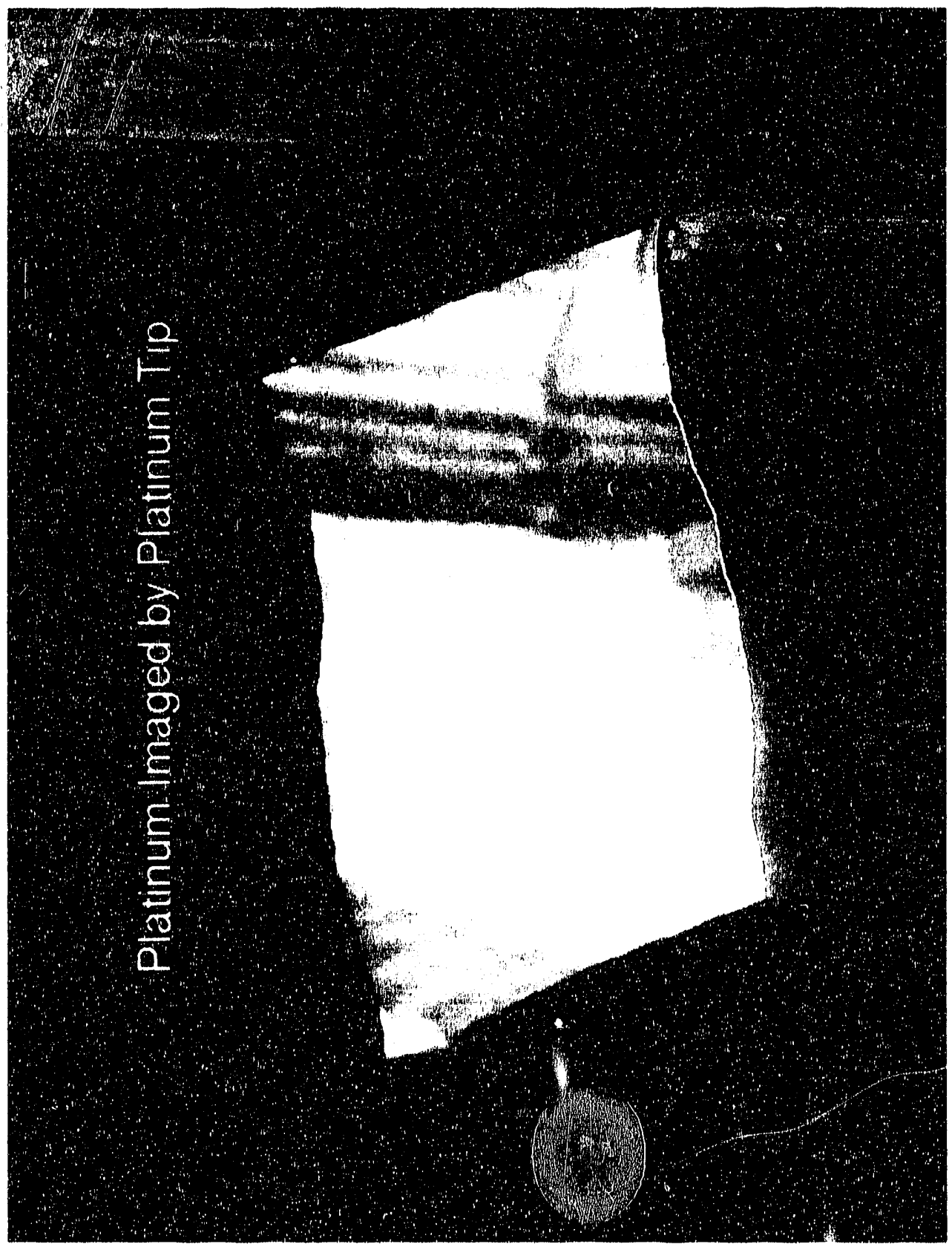


Color plate 5: Top object. closed-loop mode image of a platinum surface acquired by a tungsten tip. The image size is $100 \times 100 \mathrm{~nm}$ with a total peak-to-valley height of $82 \mathrm{~nm}$. Parameters for this data: scan time, 5 minutes; average current, $3 \mathrm{nA}$; sample bias, $0.2 \mathrm{~V}$; 8 samples/point; bias perturbation $10 \mathrm{mV}$ at $14 \mathrm{kHz}$. A 3 x 3 Gaussian convolution filter (low pass) with focus 1.0 is applied to reduce rendering noise. Bottom object. Topographic data as in the top object with texture map of the simultaneously acquired conductance signal. The conductance signal is color coded, yellow for high conductance and blue for low conductance. This means that the regions which appear bluish have a higher work function. This scene is rendered with 2 lights; data downsized by a factor of 4 and with Phong shading. 


\section{Platinum Surface With and Without}

\section{Conductance Texture}

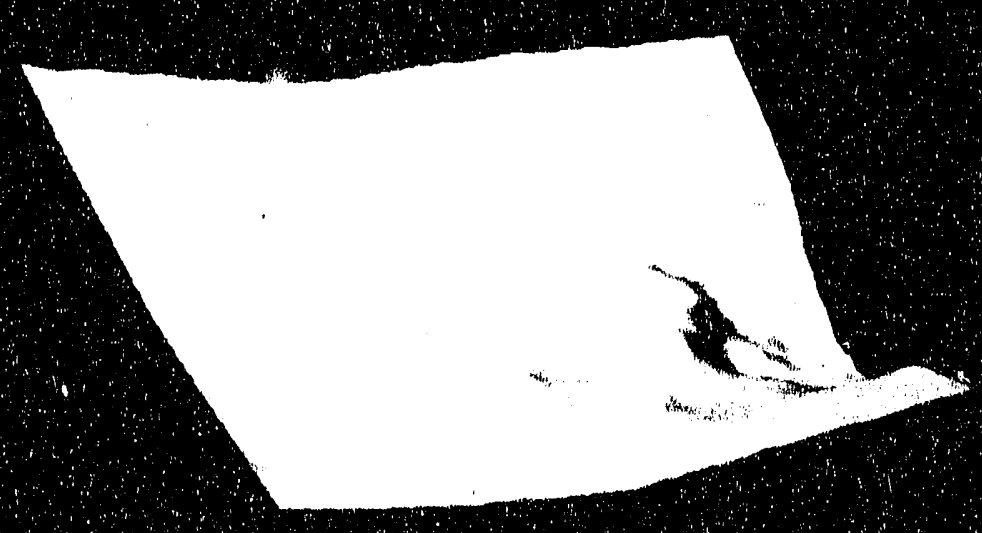


Color Plate 6: closed-loop mode topographic data and conductance texture map of HOPG acquired with a wox tip. AB in the previous color plate the conductance is color coded with yellow for tigh conductance and blue for low conductance. The image aize is $50 \times 50 \mathrm{~nm}$ with a total peak-tovalley height of $5 \mathrm{~nm}$. parameters for this data: scan time, 5 minutes; average current, $1 \mathrm{nA}$; sample bias, $-0.3 \mathrm{~V}$; 8 samples/point; bias perturbation $10 \mathrm{mV}$ at $14 \mathrm{kHz}$. A $3 \times 3 \mathrm{Ga}$ "ssian convolucion filter (low pass) with focus 1.0 is applied to reduce rendering noise. This image is rendered with 2 lights; data dornsized by a factor of 2 and with phong shading. 


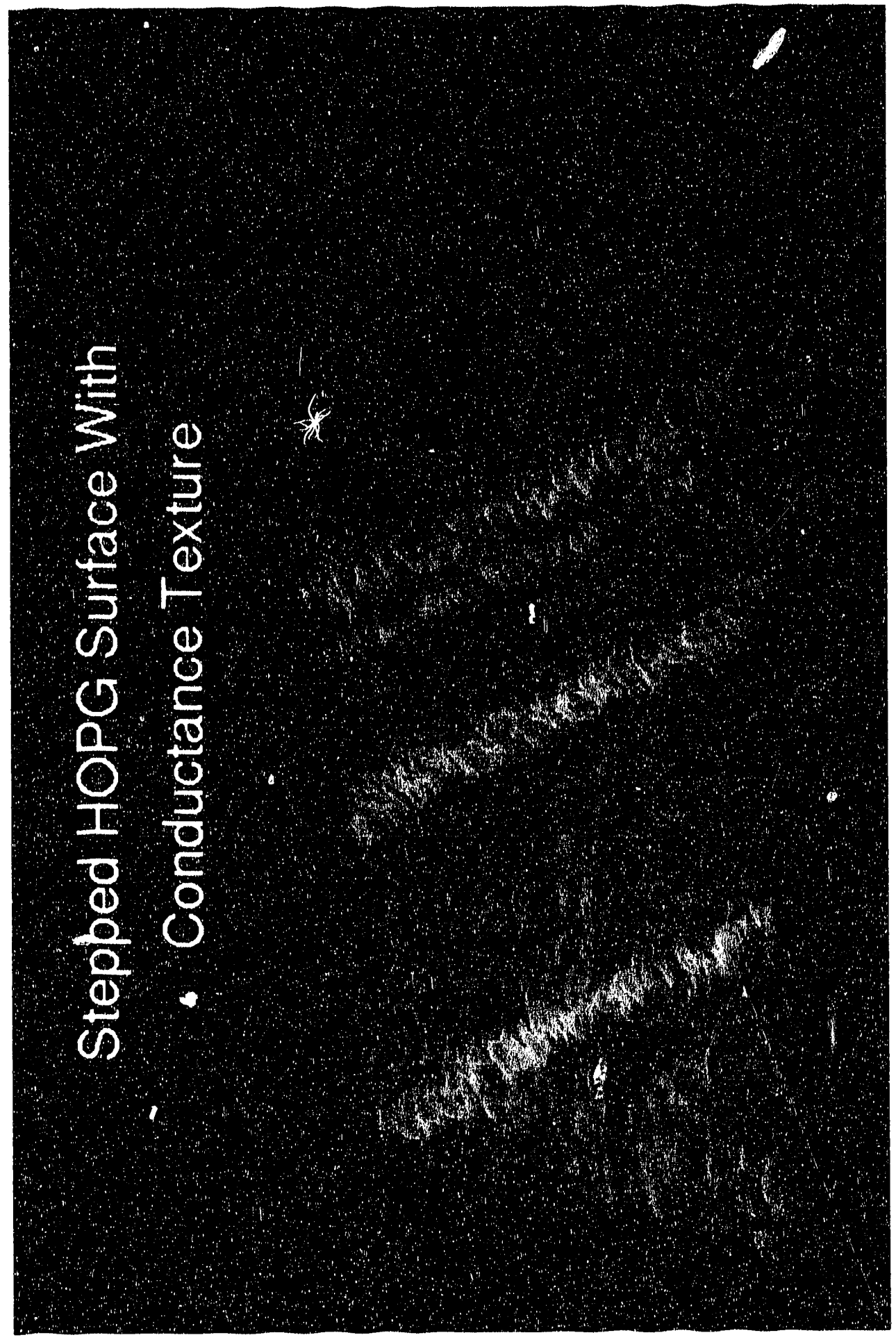


Color Plate 7: closed-100p mode image of a HOPG surface acquired with a wox tip. The image size is $50 \times 50 \mathrm{~nm}$ with a total peak-to-valley height of $6 \mathrm{~nm}$. This is a snapshot of a defect between two flat sheets of graphite. The large buckle/step features which run off in three directions from the center of the region vary in height from 2 to $4 \mathrm{~nm}$. Parameters for this data: scan time, 2 minutes; average current, 1.0 nA; sample bias, $0.2 \mathrm{~V} ; 8$ samples/point. A 3 x 3 Gaussian convolution filter (low pass) with focus 0.5 is applied to reduce rendering noise. This image is rendered with 2 liçhts; data downsized by a factor of 2 and with Phong shading. 


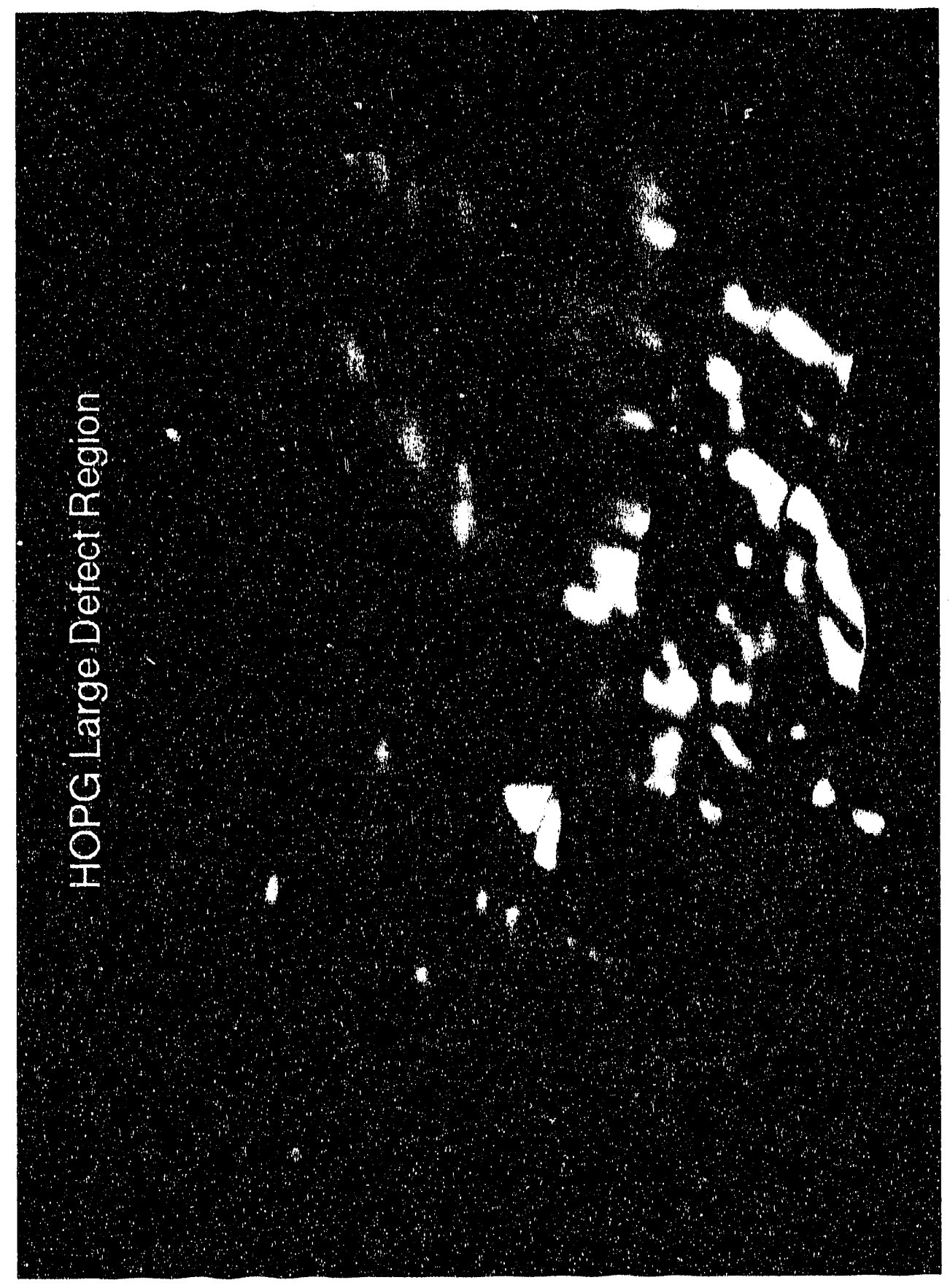


CEAPTER 4

SUMMARY

This chapter sumarizes the results and discussions presented in the previous chapters. Where possible without sacrificing brevity, quantitative results will be reiterated here; for more detail, please consult the appropriate chapter.

The synthesis and characterization of low-defect density silver/agueous silver nitrate interfaces were performed. Bowever, lowactivity surfaces similar to those reported previously by the researchers at the Bulgarian Academy of sciences were not observed. Instead, we found that capillary growth techniques produced low-index silver surfaces which were either inactive or which demonstrated activity predominantly at one defect site. Inactive silver burfaces have not been reported previously. study of the inert and slightiy active surfaces allowed determination of quantities which have previously been experimentally problematic. on the inert surfaces, impedance spectroscopy was used to study water dipole reorientation at the interface and surface adsorption of silver ad-species. The enthalpy of reorganization of water dipoles at the interface was calculated to be $24.5 \mathrm{~kJ} / \mathrm{mol}$. The typical occupation of adsorbed silver atoms is around 1 atom per 400 surface atoms at room temperature. Our observed nucleation rates obtained for the firat time at inert surfaces and the previously-reported nucleation rates for silver clusters have been 
interpreted using a model which incorporates site-dependent surface free energy terms and the stabilization of clusters at defoct sites. We find that the "typical" surface defect studied by this work and previous work stabilizes a critical cluster by a small percentage of the total surface energy of the low-index silver surface. Bowever, this 58 change produces an enhansement of up to 8 orders of magnitude in nucleation rate.

Unlike the researchers at the Bulgarian Academy of Sciences, we were apparently able to successfully prepare silver surfaces that had a mixture of active and inactive regions. The active regions contained a single defect which dominated the reactivity of that region. These defects were apparently formed at the crystal wall/capillary wall interface. After a few experiments, their presence was easy to detect. because of their impact on the total observed growth rate. Growth on these small active regions proceeded as a deterministic series of small growth events with distinctive shapes. The rate determining step in the nucleation and growth process of these events was the surface selfdiffusion of silver atoms at the interface. We determined surface diffusion coefficients for this surface moiety at two temperatures; at $T=20^{\circ} \mathrm{C}$, we found $D_{g}=(1.2 \pm 0.3) \times 10^{-7} \mathrm{~cm}^{2} \mathrm{~s}^{-1}$, and at $\mathrm{T}=50^{\circ} \mathrm{C}$, $D_{g}=(2.5 \pm 0.9) \times 10^{-7} \mathrm{~cm}^{2} \mathrm{~s}^{-1}$. One interesting direction for further study on this system would be the use of defect site location information as a guide to the study of the defect surface structure. surface sites with different cluster nucleation rates (for which there is some evidence, such as our wall defects and the sites on the "inert" 
surfaces propured by Budeveki ot al,) could be used to correlate aurface otructure with energetics and reactivity.

The computational modeling of growing surfaces was one of the more interesting parts of this thesis work. We were able to mow that computer modeling of the growth of a circular layer onto an elliptical substrate was feasible, and the resulto of these simulations wore used to develop positional analysis routines. These routines were largely acceasul in determining the nucleation site of a growth ovent given the time dependance of the growth reaction rate. For a macroscopic cryatal surface within a mall cepillary tube, the calculations showed that it is possible to locate a microscopic nucleation site with fair procision. Iypically, the mean deviation from the calculated site and the actual site was between 400 and $900 \mathrm{~nm}$. This approach is one way to bridge the gap betwean macroncopically sbserved reactivity araj microscopic probes that can tudy surface otructure. Experimentally observed growth events were modeled in a similar manner. Onedimensional hopping simulations were used to study hopping tatistice under long diffusion lengths and low concentrations from an equilibrium concentration region. A linear relation was discovered between the inverse of the simulation length and the scalirg of the apparent diffusion coefficient. This relation allowed smaller simulations, accessible to simple complaters, to accuratel.y model diffusion over macroscopic silver crystal surfaces.

ror our scanning tunneling microscope/spectrometer, the surfaces and results have spanned the range from reproducing other work to the application of simultaneous imaging technigues to platinum and EOPG. In 
this work, we applied silver cryotal growth techniques to develop a reusable tip. The availability of these eilver microcrystal tips opens possibilities for unique quality control by in-situ tip modification via cryetal growth. One extremely challenging part of working in auch a popular field as SIM is performing work which stands out as unique. We feel that by constructing our own microscope, we have been able to optimize our tool for simultaneous acquisition of topographic and conductance data. The optimization process itself proved a considerable takk during which we encountered artifacts inherent to these inetrumento that many novice users of commercially available eystems may not consider.

simultaneous imaging and visualization of combined topographic and conductance data of platinum nurfacen has provided a glimpae at auface region differences which are nominally not apparent to typical smM instruments. These ourface region differences appear as changes in local work function. These relative work function changes are around 308 over the entire surface. Spectroscopic simultaneous imaging techniques have shown local electronic changer, at steps on Bopg. The observed experimental local work function change is $1 \pm 0.5 \mathrm{v}$; this quantity io larger than expected. Bopefully, further work of this typo will lead to a better understanding of the lectronic properties of steps at surfaces and interfaces. On BOPG, terrace atacking fauits havo also been observed; again, a detection of oubtle surface differences not apparent to traditional scanning tunneling microscopy. Application of complementary techniques such as scanning conductance measurements to 
sTM-based instruments appears to be a very promising addition to the tools for study of surfacen and interfacen. 


\section{BIBLIOGRAPEY}

1. G. A. Somarjai, "Chemistry In Two Dimensions: surfaces" (Cornell University Prose, Ithaca, 1981)

2. D. King and D. P. Woodruff (Eds.), "The chemical Physics of solid Surfaces and Heterogeneous Catalysis", vol. 5, (Elsvier, New York, 1988) 3. W. K. Burton, N. Cabrera, and F. C. Frank, Phil. Trans. Roy. Soc. A $243,299-358(1951)$

4. B. Iewis, Nucleation and Growth Theory, in B. R. Pamplin (ed.), "Crystal Growth" (Pergamon Press, Oxford, 1980)

5. S. Fletcher and T. Iwin, Electrochim. Acta 28(2), 237-243, (1983)

6. A. Milchev, Electrochim. Acta 28(7), 947-953 (1963)

7. R. I. Deutacher and S. Fletcher, J, Electroanal. Chem. 23y, 17-54 $(1988)$

8. J. D. Porter, Doctoral Thesis, Carlton University, (1981)

9. M. B. J. Hottenhuis, A. I. M. van der Berg, and J. P. van der Eerden, Electrochim. Acta 33(11), 1519-1538 (1988)

10. V. Bostanov, W. Obretenov, G. Staikov, and E. Budevski, J. Electroanal. Chem. 246, 303-312 (1983)

11. J. W. Gibbs, "on The Equilibrium of Heterogeneous surfacea; collected WorkB", (IJongman's Green, New York, 1928)

12. E. Bauer, Appl. Surf. Sci. 11/12, 479-494 (1982)

13. T. N. Taylor, R. E. Muenchausen, and M. A. Boffbauer, surf. Sci. 243 $65-82(1991)$ 
14. A. J. Bard, R. Parsons, and J. Jordan (eds.), "standard Potentials in Agueous solution" (Marcel Dekker, New York, 1985)

15. C. Cachet, M. Froment, M. Reddam, and R. Wiart, Electrochim. Acta 21. $879-888(1976)$

16. J. Verrecken and R. Winand, Electrochim. Acta 22, 401-409 (1977)

17. C Cachet, G. Gabrielli, F. Eurt, M. Keddam, and R. Wiart, Electrochion. Acta 28(7), 899-908 (1983)

18. M. B. J. Bottenhuis, M. A. B. Hickers, J. W. Gerritson and J. P. van der Eerden, Surf. Sci. 206, 259-278 (1988)

19. C. Franke, G. Piazza, and D. M. Kolb, Electrochim. Acta 34(1), 67-73 (1989)

20. R. S. Roluinson, J. Vac. Sci. Technol. A 8(1), 511-514 (1990)

21. R. Raschev and E. Budevski, Contemp. Phys, 8(5), 489-516 (1967)

22. C. N. Nanev and D. Vladikova, Electrochim. Acta 23, 325-329 (1978)

23. C. N. Nanev, Electrochim. Acta 25, $1383-1387$ (1980)

24. W. Obretenov, V. Bostanov, and V. Popov, J. Electroanal. Chem. 132, $273-276(1982)$

25. V. Bostanov, W Obretenov, G. Staikov, and E. Budeveki, J. Electroanal chem. 146, 303-312 (1983)

26. R. G. Baxradas, P. T. Boag, J. D. Porter, T. J. Vandernoot, E. Burlevski, V. Bostanov, w. Obretenov, and T. Koesev, J. Electroanal. Chem. $154,1-12(1983)$

27. W. Obretenov, V. Bostanov, and E. Budevaki, J. Electroanal. Chem. $170,51-62(1984)$

28. E. Budevaki, w. Obretenov, V. Bostanov, G. Staikov, J. Doneit, X. Juttner, and W. J. Lorenz, Electrochim Acta 34(8), 1023-1029 (1989) 
29. v. Bostanov and w, Obretenov, Electrochim. Acta 34(8) 1193-1196 (1989)

30. J. R. Macdonald, "Impedance Spectroscopy", (Wiley, New York, 1987) 31. A. J. Bard and I. R. Faulkner, "Electrochemical Methods", (Wiley, New York, 1980)

32. T. P. Van Doren, workbook: "Grounding and Shielding Electronic Instrumentation" (Department of Electrical Engineering, Univ. of Missouri-Rolla, 1985 )

33. P. Horowitz and w. Eill, "The Art of Electronics", second edition, (Cambridge University Pres8, Cambridge, 1989)

34. w. F. Smith, "Principles of Materiale Science and Engineering", (KcGraw-Hil1, New York, 1986), P. 266

35. D. C. Grahame, Chem. Rev, 41, 441-501 (1947)

36. R. C. Weast, ed, "CRC Eandbook of Chemistry and Physics", 63rd edition, (CRC Press, Boca Raton, 1982), E-E1

37. A discussion of chemical oscillators can be found in; $B$. Degn, J. Chem. Ed. 49, 302-306 (1972), a historical discussion of the Belousovzhabotinski. reaction is given by; A. T. Winfree, J. Chem. Ed. 61, 661$663(1984)$

38. P. W. Atkins, "Physical Chamistry", second edition, (Froman, san Francisco, 1982), chapter 26

39. M. R. Mruzik and G. M. Pound, J. Phys. F 11, 1403-1422 (1981)

40. J. D. Doll and B. R. MCDowell, J. Chem. Phys. I7(1), 479-483 (1982)

41. G. Ehrlich and K. Stolt, Ann. Rev. Phys. Chem. 31, 603-637 (1980)

42. T. Tsong, Surf. Sci. Rep. $8(3-4), 127-209$ (1988)

43. P. A. Thiel and T. E. Madley, surf. Sci. Rep. $Z(6-8), 211-385$ (1987) 
44. R. G. Barradas and J. D. Porter in R. Weil and R. G. Barradas (eds), "Electrocrystallization", (The Electrochemical Society, Pennington, $1987)$, P. 57

45. M. Avrami, J. Cham. Phys. 8, 212-224 (1940)

46. M. Avrami, J. Chem. Phys. 2, 177-184 (1941)

47. M. Fleischmann and B. R. Thrisk in P. Delahay and C. Tobias (eds) "Advances in Electrochemistry and Electrochemical Engineering", vol. 3, (Wiley Interscience, New York, 1963)

48. R. G. Barradas and T. J. Vandernoot, J. Electroanal, Chem. 142, 107$119(1982)$

49. R. G. Barradas and T. J. Vandernoot, J. Electroanal. Chem. 176, 151167 (1984)

50. A. Sinith and S. Fletcher, Electrochim. Acta 25, 889-897 (1980)

51. G. Binnig, C. F. Quate, and Ch. Gerber, Phys. Rev. Lett. 56, 930-933 $(1986)$

52. G. Binnig, B. Rohrer, Ch. Garber, and E. Weibel, Appl. Phys. Lett. 40, $178-180(1982)$

53. D. A. Reed and G. Ehrlich, Surf. Sci. 102, 588-609 (1981)

54. D. A. Reed and G. Ehrlich, Surf. Sci. 105, 603-628 (1981)

55. M. Bowkex and D. A. King, surf. Sci. Z1, 583-598 (1978)

56. W. H. Press, B. P. Flannery, S. A. Teukolsky, and W. T. Vetterling, "Numerical Recipies: The Art of scientific Computing", (Cambridge University PresB, Cambridge, 1986), p. 195

57. G. Binnig and 8 . Rohrer, IBM J. Res. Dev. 30, 355-369 (1986)

58. J. A. Golovchenko, science 232, 48-53 (1986)

59. T. E. Feuchtwang and P. H. Cutler, Phys. Scr. 35 132-140 (1987) 
60. R. J. Bamers, Ann. Rev. Phys. Chem. 4l, 531-559 (1989)

61. 日. Strecker and G. Persch, Appl. Surf. Sci. 46(1-4), 441-445 (1990)

62. J. E. Griffith and G. P. Kochanski, Ann. Rev. Matr. Sci. 20, 219-224 (1990)

63. P. K. Hansma, "Tunneling Spectroscopy: Capabilities, Applications, and New Techniques", (Plenum Press, New York, 1982)

64. E. I. Wolf, "Principles of Electron Tunneling spectroscopy", International series of Monographs on Physics 21, (Oxford University Pres8, New York, 1985)

65. I. Esaki, Phys. Rev. 109, 603-604 (1958)

66. J. C. Fisher and I. Giaever, J. Appl. Phys. 32, 172-177 (1961)

67. R. B. Good and E. W. Müller, Eandbuch der Physik 21, 176-231 (1956)

68. J. Tersoff and D. R. Bamann, Phys. Rev. B 31(2), 805-813 (1985)

69. J. Bardeen, Phys. Rev. Lert. 6, 57-59 (1961)

70. C. J: Chen, J. Vac. Sci. Technol. A 6(2), 319-322 (1988)

71. D. M. Eiglex, C. P. Lutz, and W. E. Rudge, Nature 352, 600-603

(1991)

72. J. A. Stroßcio, R. M. Feenstra, and A. P. Fein, Phys. Rev. Lett. 57 , $2579-2582(1986)$

73. N. D. Lang, Phys. Rev. B 34(8), 5947-5950 (1986)

74. J. Schneir, R. Sornenfe]d, O. Marti, P. K. Hansma, J. E. Demuth, and R. J. Bamers, J. Appl. Phys. 63(3), 717-721 (1988)

75. H. Strecker, C. Stahl, and B. Starke, J. Vac. Sci. Tochnol. A (1), $618-620(1990)$

76. T. Kansma, personal communication 
77. R. Nicolades, Y. Liang, W. E. Packard, Z. -W. Fu, B. A. Blackstead, K. K. Chin, J. D. Dow, J. K. Furduna, w. M. Bu, R. C. Jaklevic, W. J. Raiser, A. R. Pelton, M. V. Zeller, and J. Bellina Jr., J. Vac. Sci. rechnol. $6(2), 445-447(1988)$

78. J. Garnaes, F. Kragh, K. A. Morch, and J. R. Thölén, J. Vac. Seil. Technol. A $8(1), 441-444$ (1990)

79. J. McBardy and P. Stonehart, in J. O'M. Bockris (Ed.), International Review of science, Physical Chemistry, series 2, vol. 6 , "Electrochemistry", (1976), pp, 171-229

80. C. A. Goss, D. B. Charych, and M. Majda, submitted to Analytical Notes

81. A. Moore, Union Carbide

82. W. J. Kaiser and R. C. Jaklevic, IBM J. Res. Dev. 3l(4) 411,-416 (1986)

83. A. Humbert, F Salvan, and C. Mouttet, Surf. Sci. 181, 307-312 (1987)

84. R. S. Becker, T. Rlitsner, and J. S. Vickers, Phys. Rev. B $38(5)$, $3537-3540(1988)$

85. Ph. Avoris, J. Phys. Chem. 24, 2246-2256 (1990)

86. J. Shiang, personal communication

87. L. E. Brus, J. Chem. Phys. 80, 4403-4409 (1984)

88. I. E. Brus, J. Chem. Phys. 20, 2555-2560 (1986)

89. V. I. Colvin, porsonal communication

90. Y. Ruk and P. J. Silverman, J. Vac. Sci. Technol. A(1), 289-292 (1990)

91. Reference 1, page 248

92. J. I. Gland and G. A. Somorjai, Surf. Sci. 38, 157-186 (1975) 
93. N. D. Lang and W. Kohn, Phyo. Rev. B I(12), 4555-4568 (1970)

94. N. D. Lang and W. Kohn, Phys. Rev. B 3(4), 1215-1223 (1971)

95. for a general discussion see: A. Zangwill, "Physics at surfaces", (Cambridge University Prass, Cambridge, 1988), pp. 57-63

96. M. D. Thompson and B. B. Buntington, surf. Sci. 116, 522-37 (1982)

97. K. Besocke, B. Krahl-Urban, and B. Wagner, surf. Sci. 68, 39-46 (1977)

98. Y.-W. Yu and J. D. Porter, unpublished result

99. G. S. Painter and D. E. Ellis, Phys. Rev. B 1(12), 4747-4752 (1970) 
source code for silver growth experiments:

GROWSAMP BAS source code:

WRRSPCE BAS gource code:

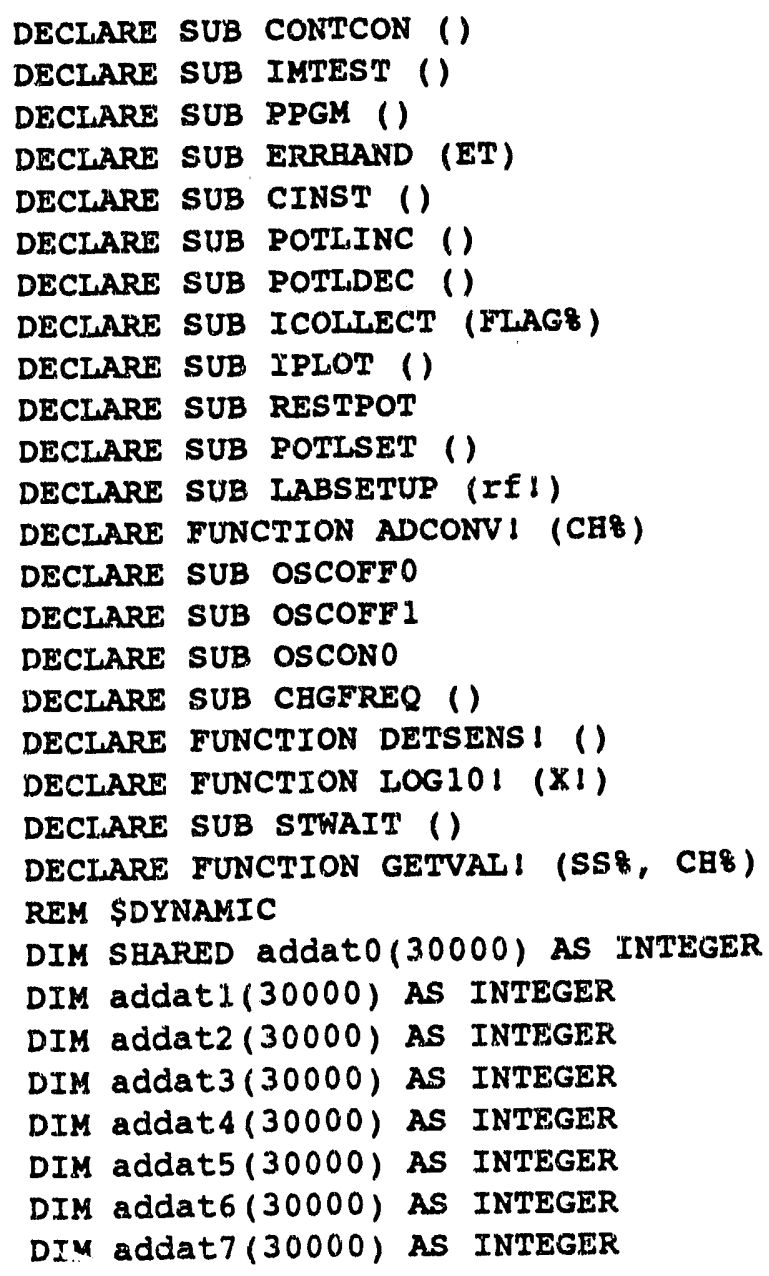


'Impedence scans and double pulse experiments can be conducted.

'the main module is the option module while the impedence scan program

'is in another module, IKTEST.

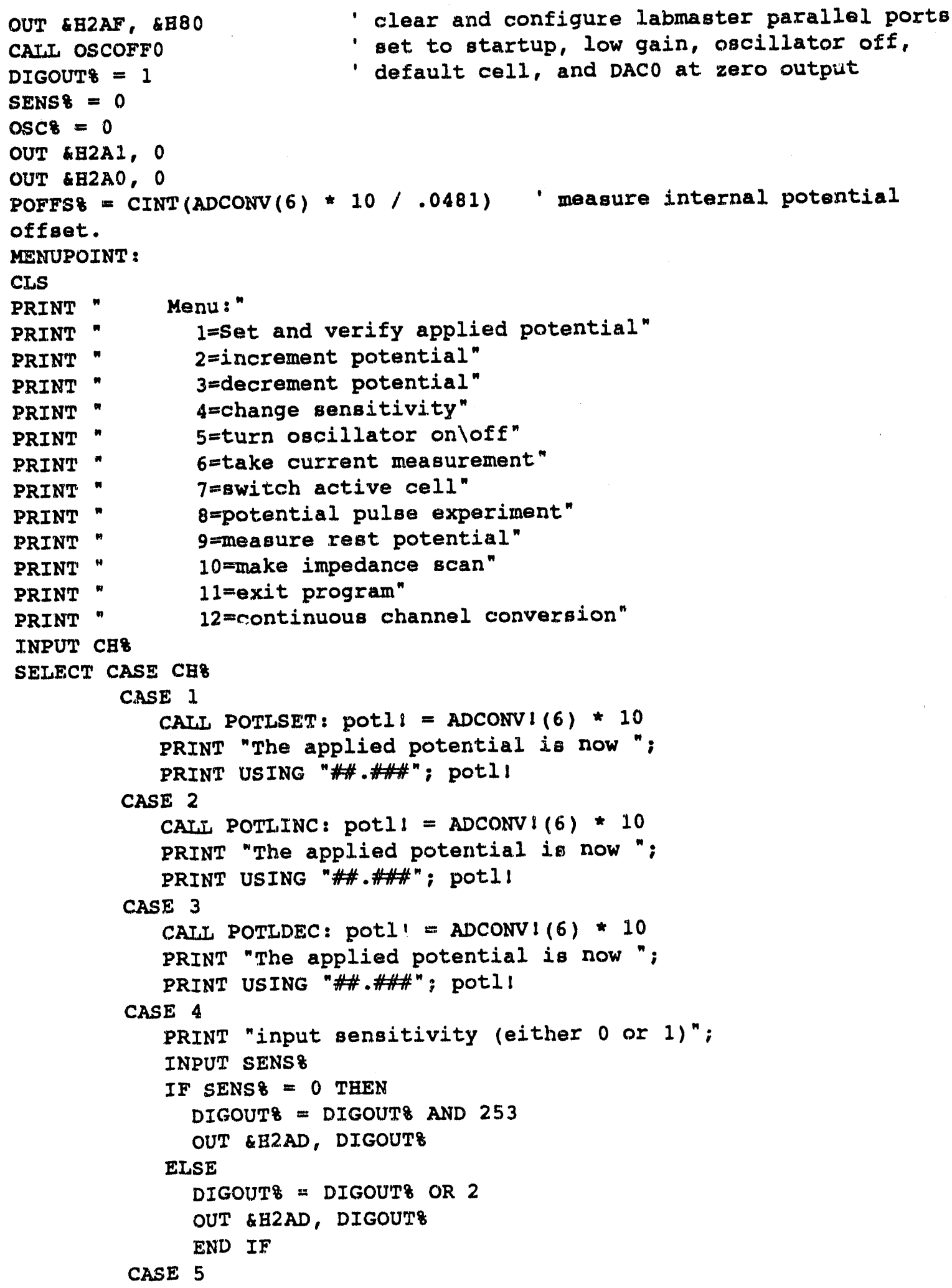




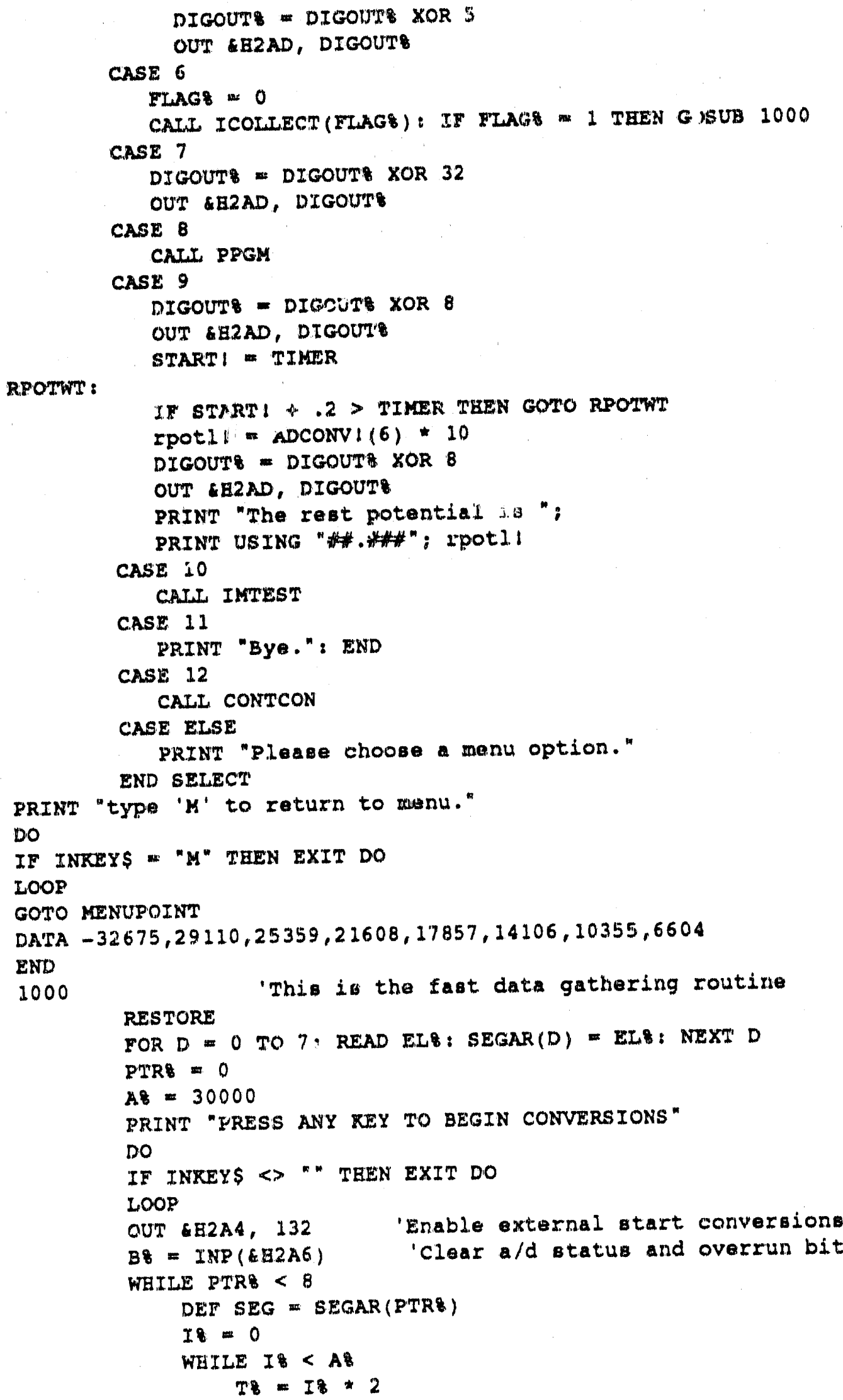

R.POTWT: 
$\operatorname{INP}(\mathrm{BH} 2 \mathrm{AC})$

If $\operatorname{INP}(6 E 2 A 4)>65$ THEN PRINT I8; " POINT MISSED.": B=

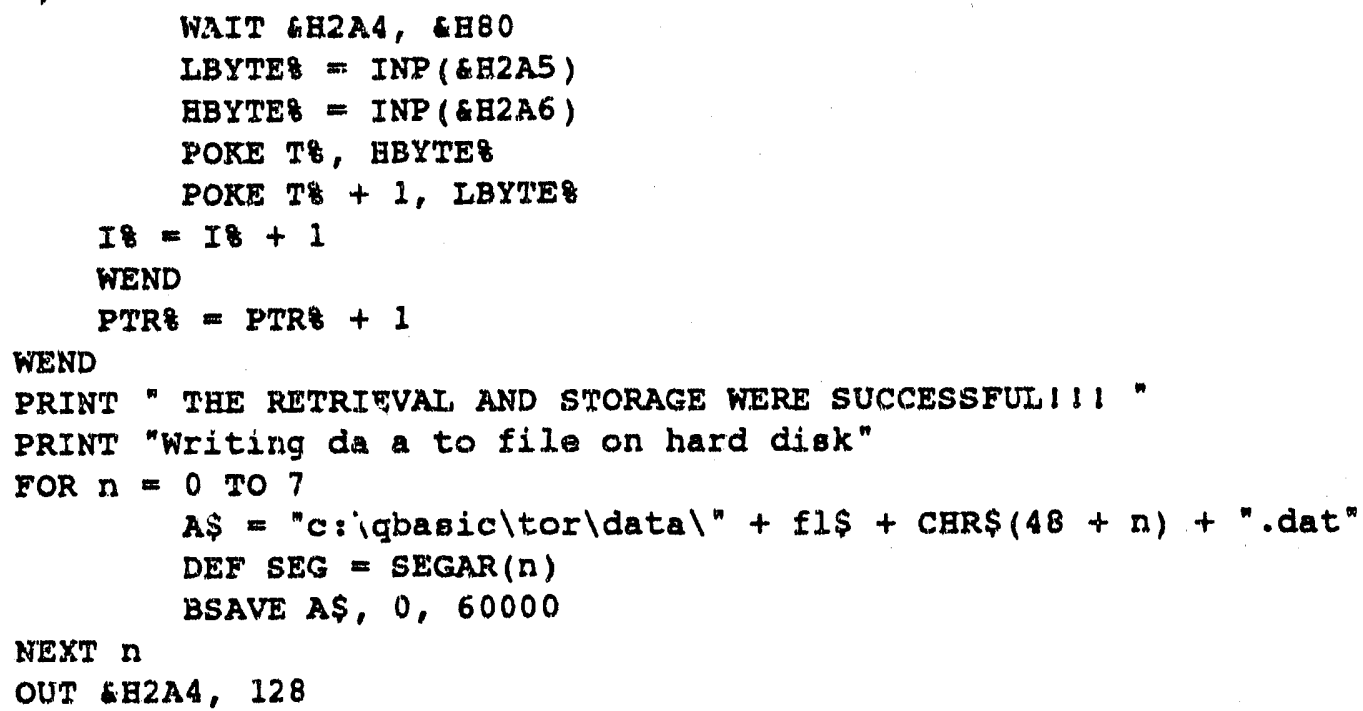

RETURN

REM SSTATIC

DEFSNG $\mathrm{A}-\mathrm{Z}$

SUB CINST

PRINT "This program can record up to 240000 points of data at user opecified rates."

PRINT "Data can be stored in single files up to 30000 points. total of. 8 files"

FRINT "can be taken at any given tine. The filename is user specified and final digit"

PRIiTt "is appended on the filenane (from 0 to 7 ). Selection of sampling rates is by "

PRINT "powers of ten only. If you want sampling at $10 \mathrm{kBz}$, then you ran't choose a file"

PRINT "length or number of files. The default is 30000 points per file and 8 files."

END SUB

DEFINT A-Z

SUB CONTCON

INPUT "CEANNEL TO CONVERT"; CH8

DO WEILE INKEYS $<$ " $Q$ "

CONTVAI! = ADCONV $(\mathrm{CB} 8)$

RRINT CONTVAL'

FOR II6 = I TO 500: II = I 16 ^ 2: NEXI II\&

LOOP

END SUB

DEFSNG $A-Z$

SUB ERRRAND (ERTYPE)

SELECT CASE ERTYPE

CASE 1

BEEP : BEEP 
PRINT "OOPS, sorry I'm not that fazt right now." PRINT "DO you wiBh to continue $(y / n)$ "; : INPUT AS IF UCASES(AS) $=$ "N" THEN END

\section{CASE LELSE}

BEEP : BEEP : BEEP

PRINT "UNKNOWN ERROR, execution stopped."

CLOSE \#1

END

END SELEC $f$

END SUB

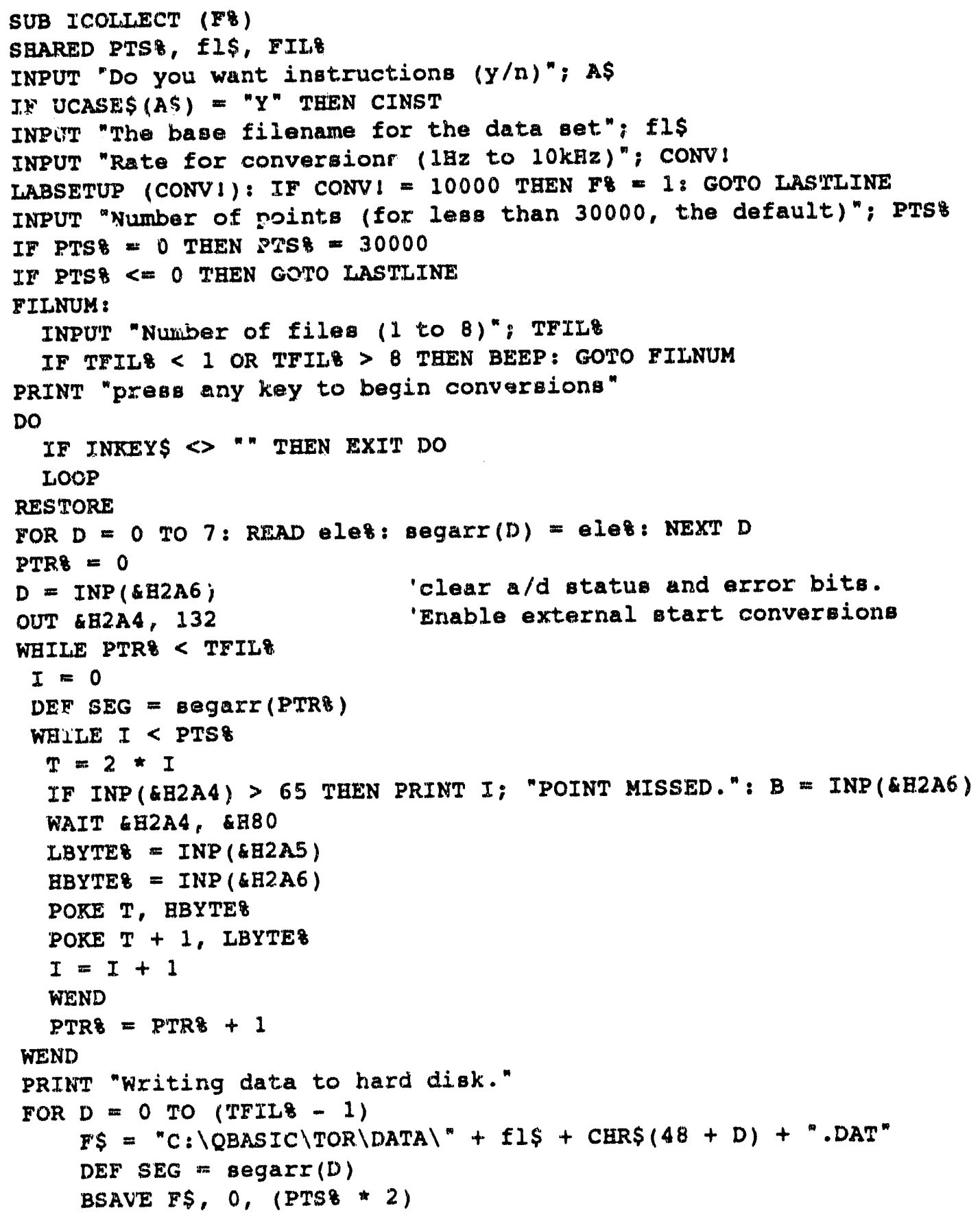


NEXT D

OUT \&B2A4, 128

IASTLINE:

END SUB

SUB IAASETUP (If!)

$A D D R=\$ 2 A O$

OUT ADDR + 4, 128 'Disable auto-increment, load enable.

OUT ADDR + 9, 23 'Set data ptr to Master Mode Register.

OUT ADDR + 8, 0 'set MMR for BCD div, 8-bit bus, comparators and

OUT ADDR + 8, 228 'TOD disabled, low byte written first.

OUT ADDR + 9,5 'set data ptr to counter 5 command reg.

OUT ADDR + 8,49 Mode D, TC toggeled output, BCD count down.

FREQ: PRINT If !

SELECT CASE If !

CASE 1

User has chosen 1 az sampling race.

OUT ADDR + 8,15 'select F5 as counter source input.

CASE 10 'User has chosen $10 \mathrm{kz}$ sampling rate.

OUT ADDR + 8, 14 'select F4 as counter source input.

CASE 100 'You get the idea.

OUT $A D D R+8,13$ 'F3

CASE 1000

OUT $A D D R+8,12$ 'F2

CASE 10000

OUT ADDR + 8, 11 F1 $1.00 \mathrm{MHz}$ Oscililator.

CASE ELSE

PRINT "Ok bozo, try factors of 10 up to $10 \mathrm{kBz}$ ":

INPUT If

GOTO FREQ

END SELECT

OUT ADDR + 8, 153 'Load counter register with decimal 99,

OUT ADDR $+8,0 \quad 153 \mathrm{BCD}=99$ deciunal.

OUT $A D D R+9,112$ 'Load counter 5 and arm

OUT ADDR $+5,7$ 'Write channel number to board.

END SUB

SUBB POTLDEC

TOT' = DPOT: - 1

HDC8 $=I N T(D P O T \& / 256)$

LDCQ $=$ DPOT\& $-($ HDCE * 256)

OUT \&H2A1, BDCE

OUT $\angle E 2 A O, I D C 8$

END SUB

SUB POTLINC

DPOT: $=$ DPOT: +1

BDC\& $=\operatorname{INT}(D P O T / 256)$

LDC8 = DPOT8 - (EDC8 * 256)

OUT \&H2A1, $\mathrm{ADC} 8$

OUT \&B2AO, LDC\&

END SUE

SUB POTLSET 


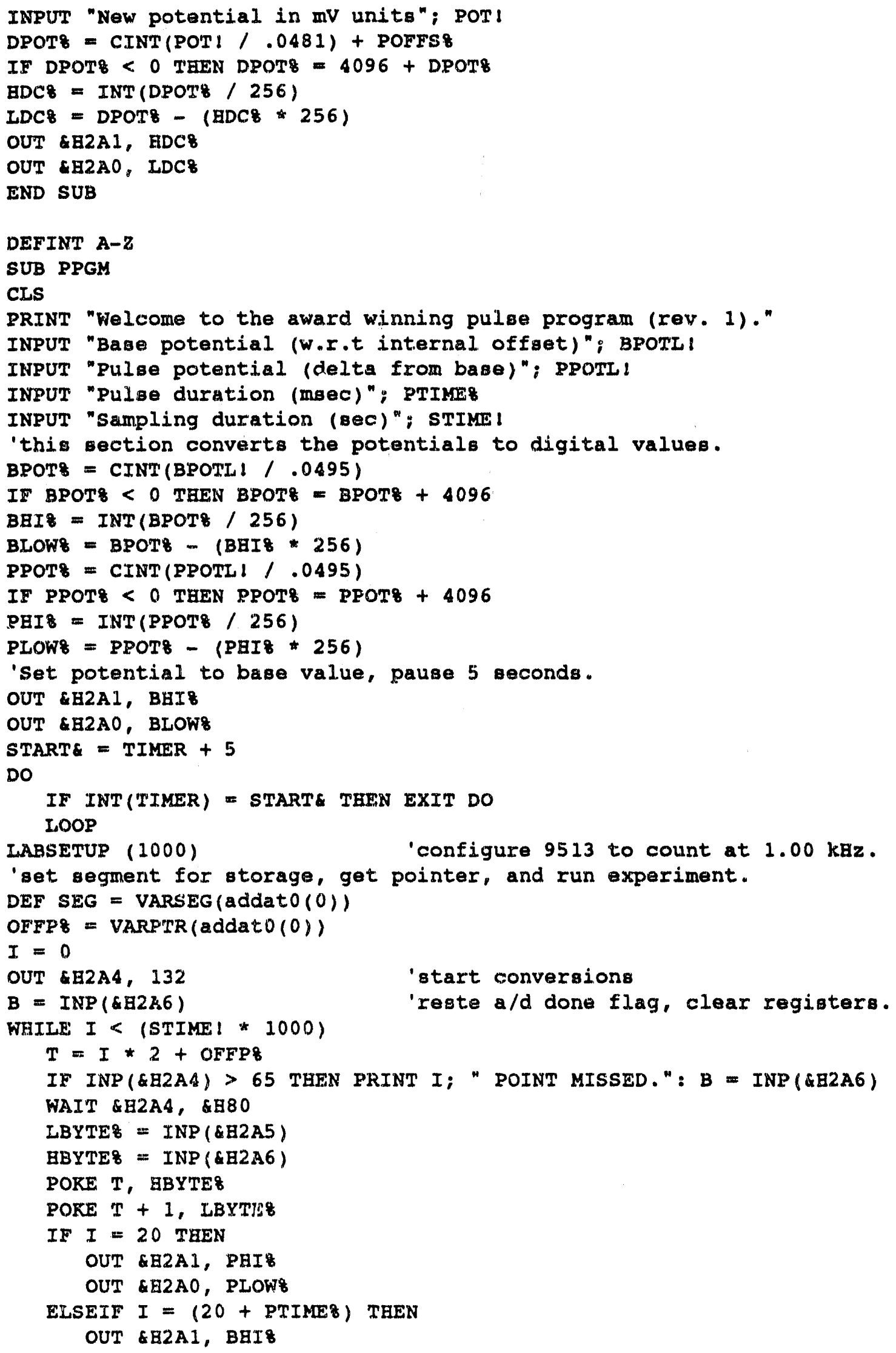




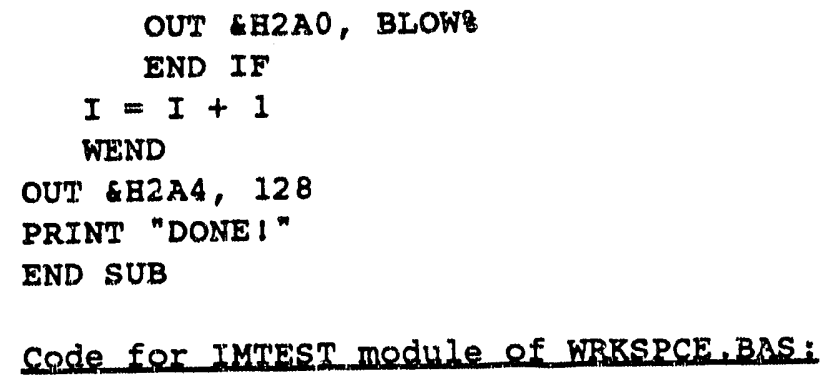




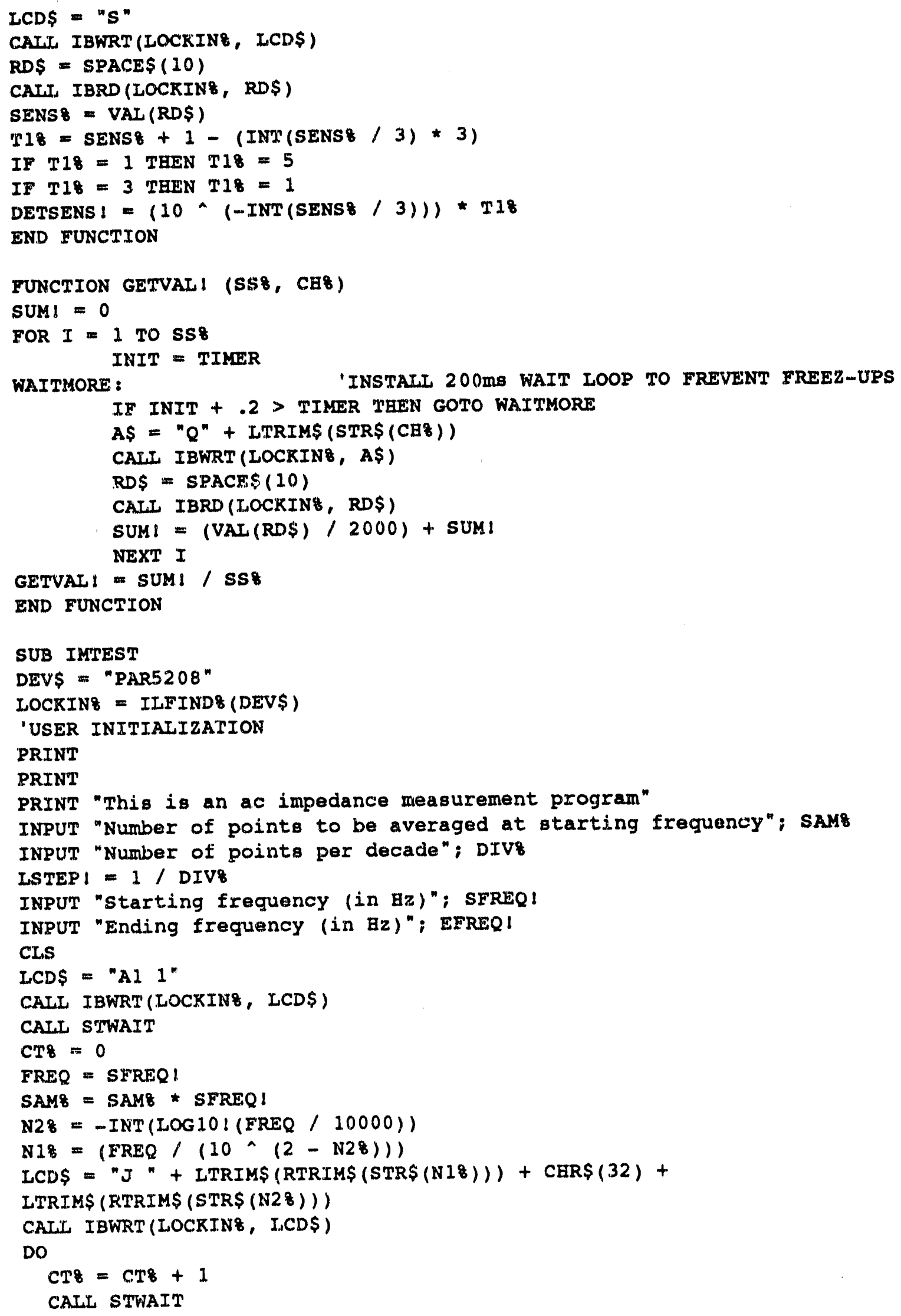




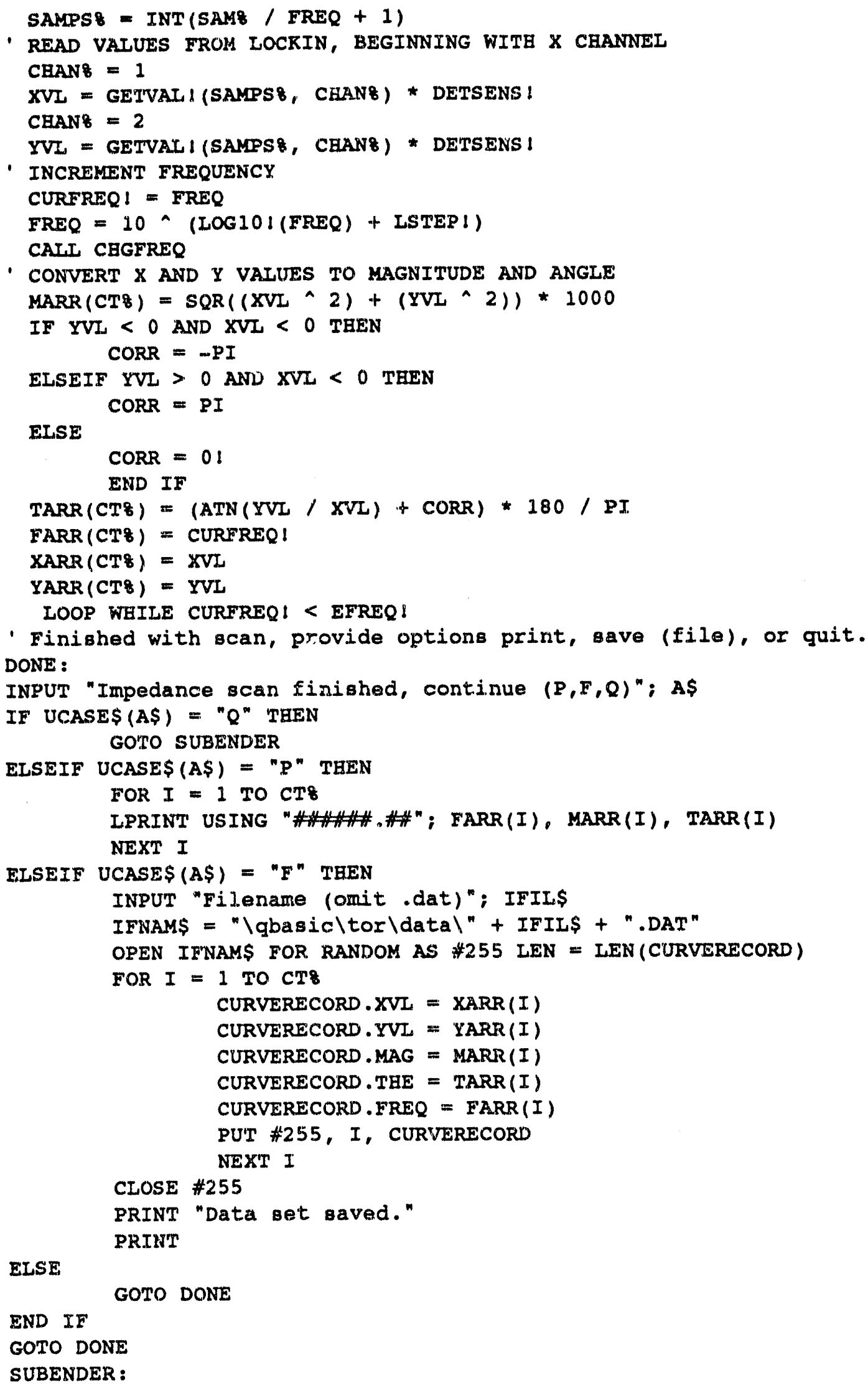




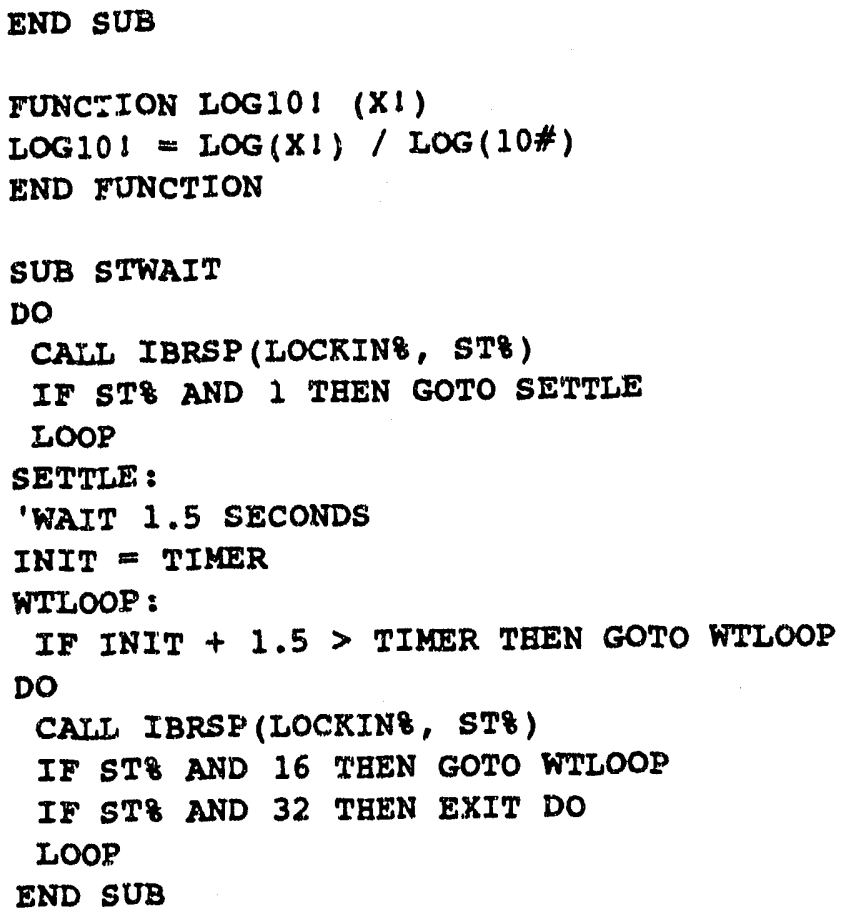




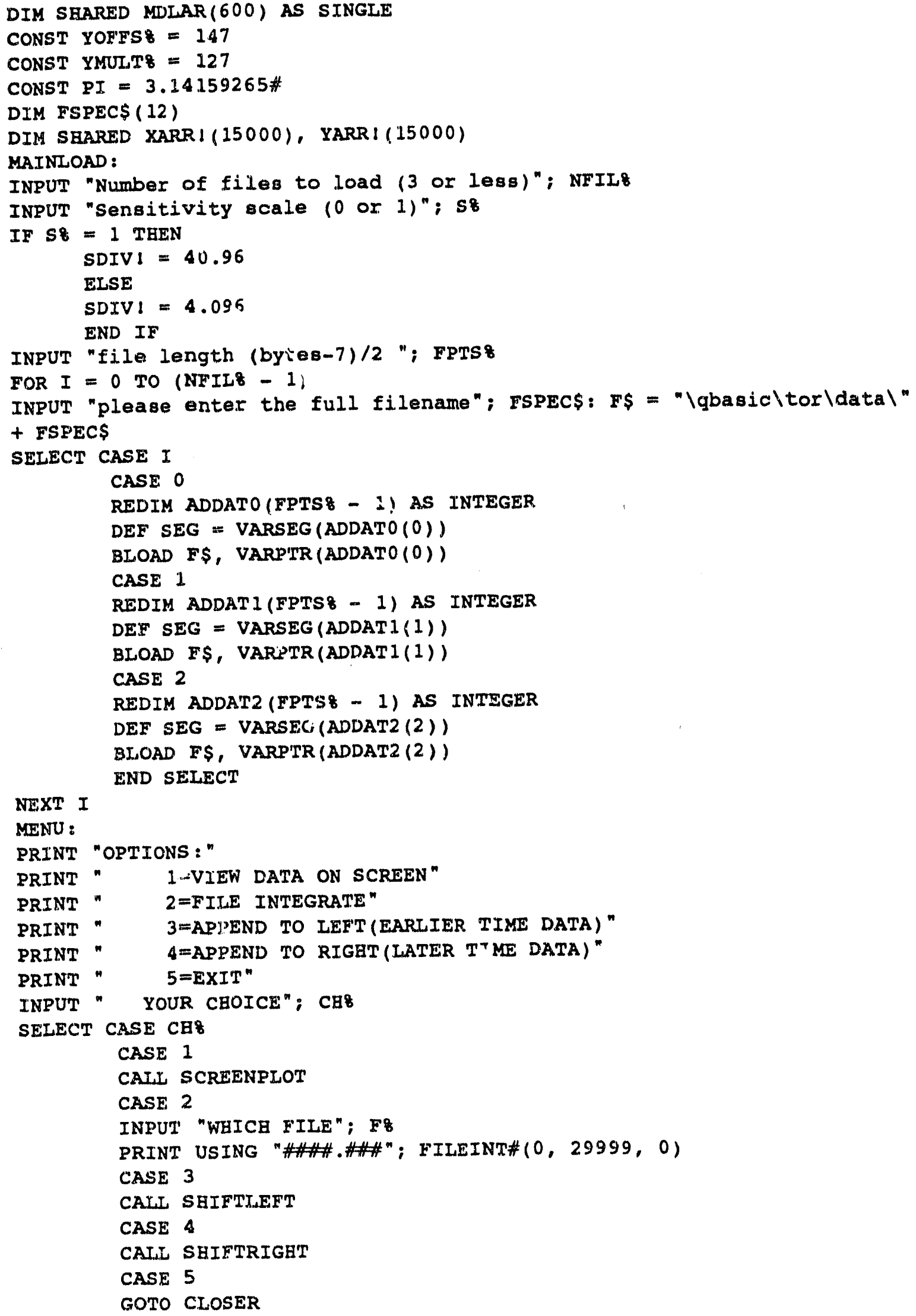




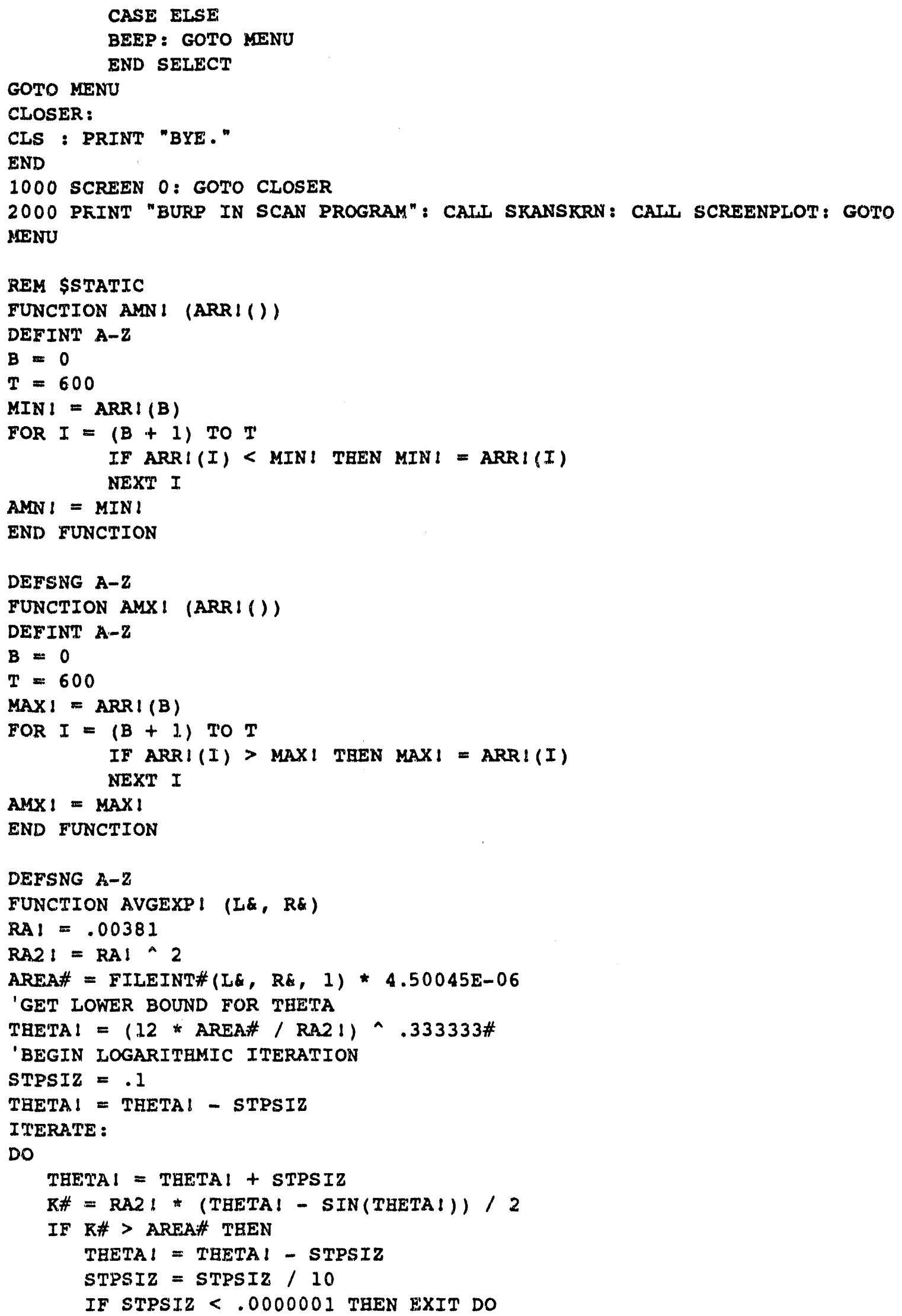




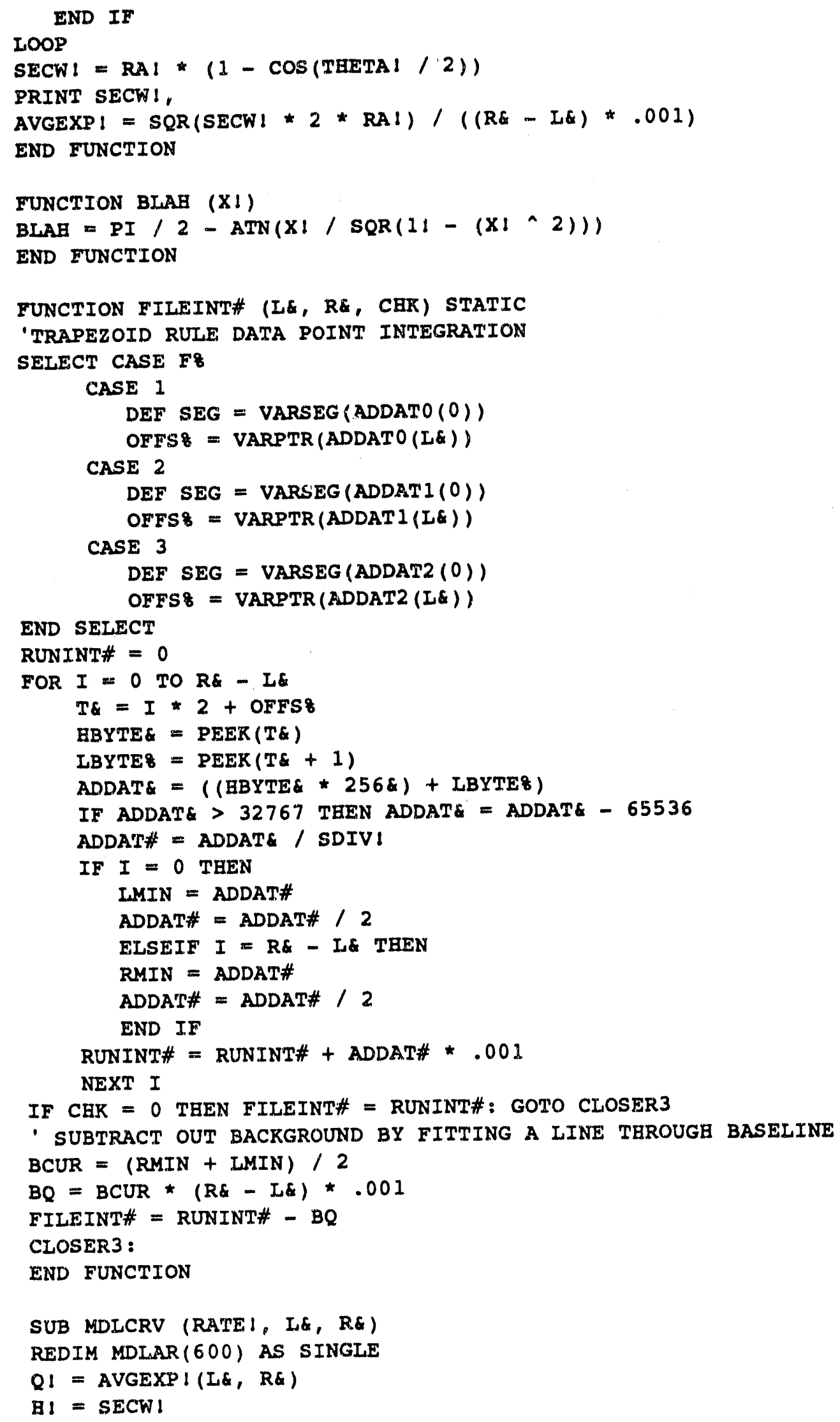




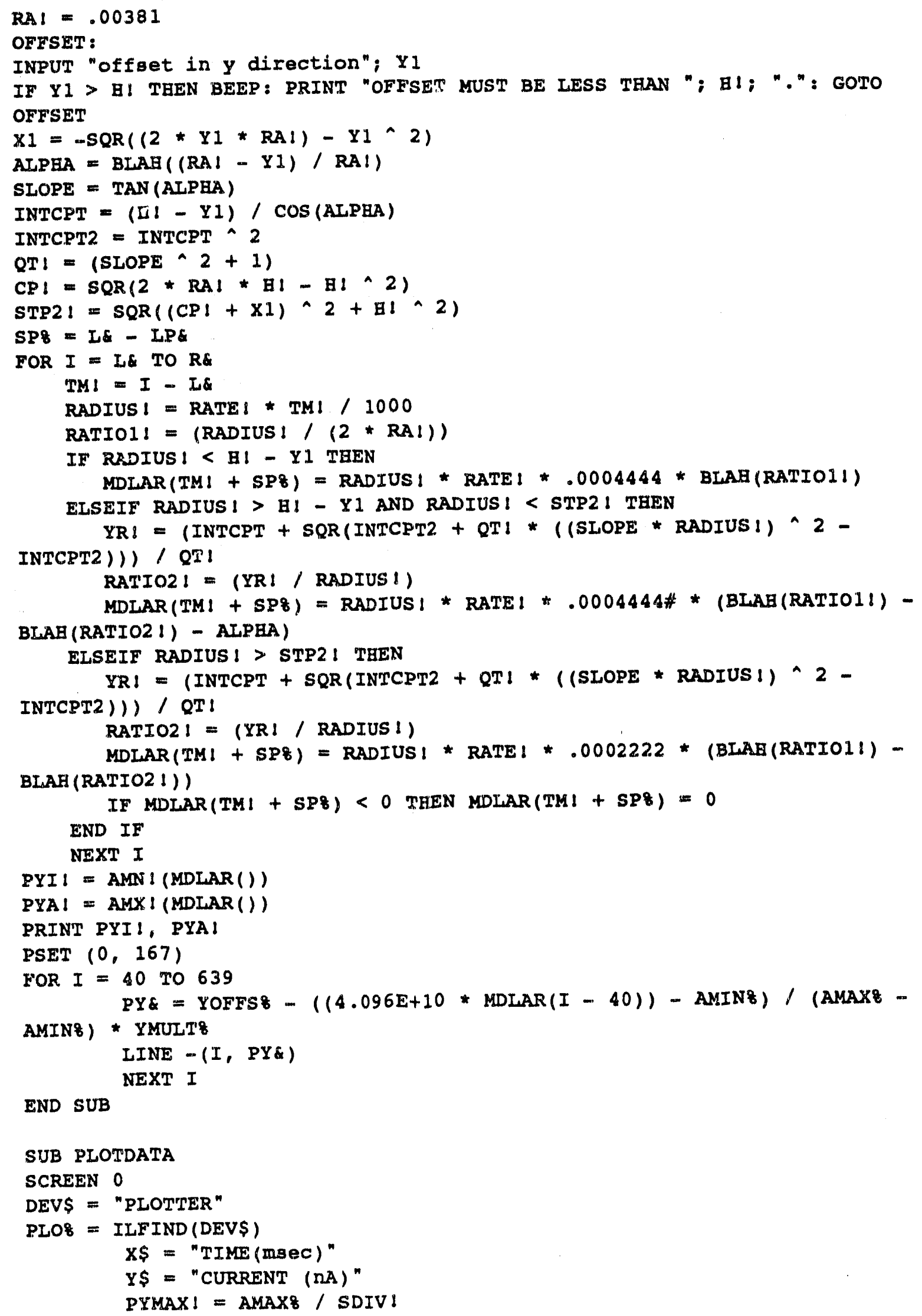

END SUB

SUB PLOTDATA

SCREEN 0

DEVS = "PLOTTER"

PLO\& = ILFIND (DEV\$)

$X S=$ "TIME (msec)"

$Y \$=$ "CURRENT (nA)"

PYMAX! = AMAX\& / SDIV! 


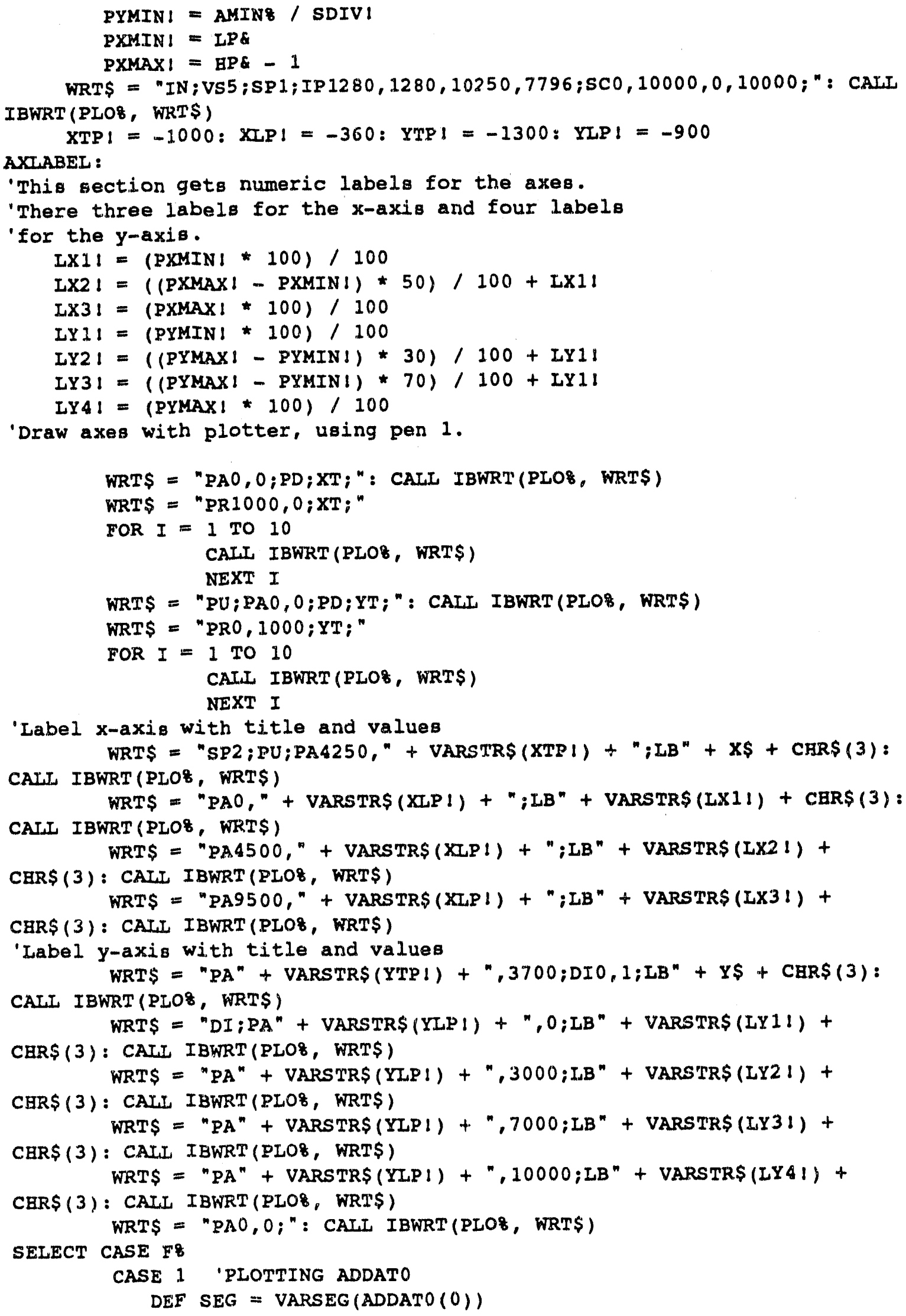




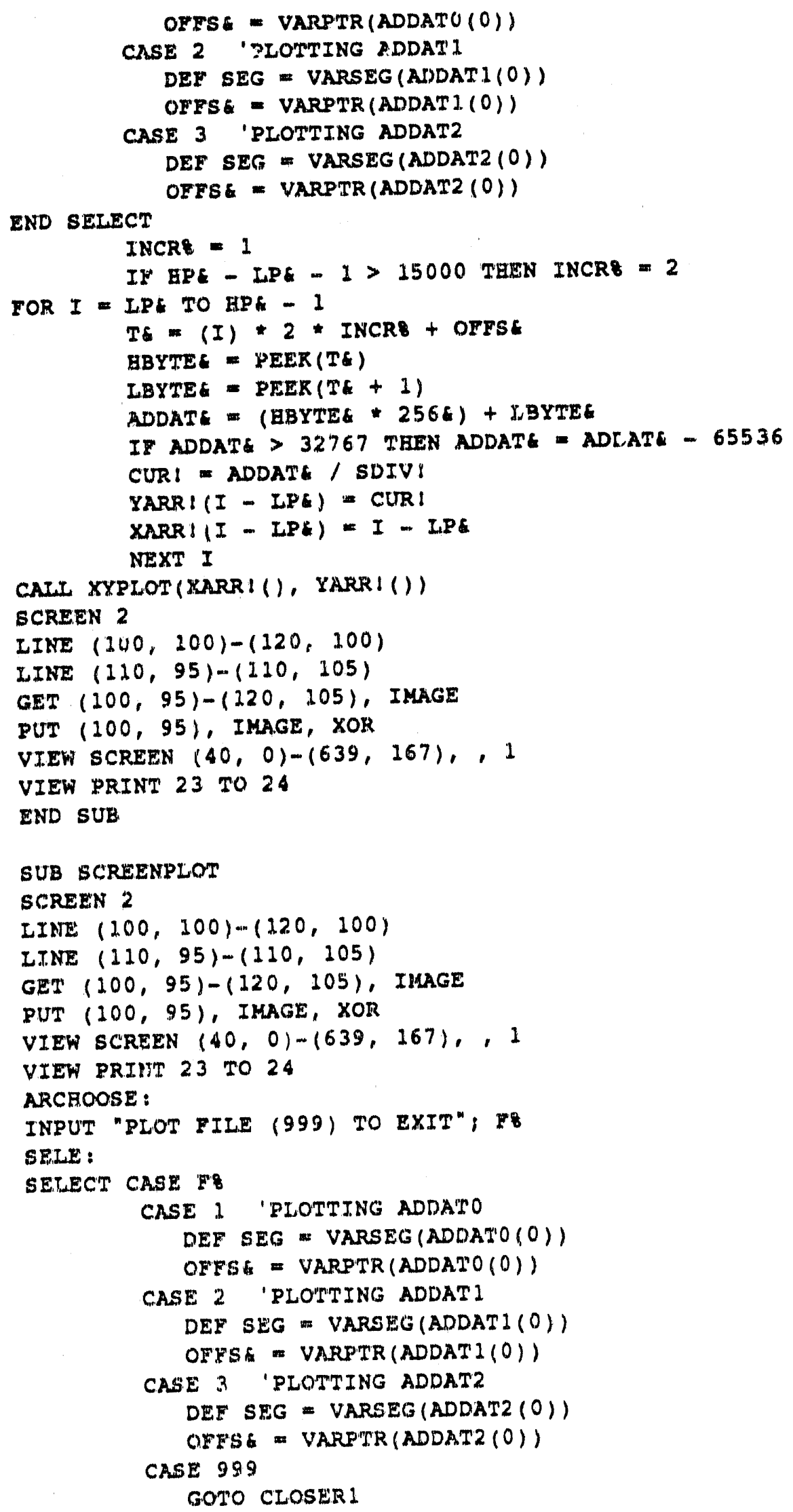


CASE EISE

INPUT "SELECT FILE (1-3) OR 999 TO EXIT"; F\&: GOTO SELE END SELECT

INPUT "POINTS PER PIXEL"; PPPE

INPUT "STARTING POINT"; SPE

IPE $=$ SPE -1

BPE = LPE + (PPPE *600)

IF AP\& > FPTS\& - 1 THEN EP\& = FPTS\& -1

CONVERT:

AMAX8 $=1$

AMINE $=5000$

FOR I = LP\& TO (BP\& - 1) STEP PPP

$T \&=2 * I+$ OFFS $\&$

BBYTE\& $=\operatorname{PEER}(T \&)$

IBYTE\& = PEER (TE + 1)

ADDATE = (BBYTE\& * 256) + IBYTES

IF ADDAT\& > 32767 THEN ADDAT\& = ADDAT\& - 65536

$A D C 8=\operatorname{CINT}($ ADDATE)

PLOTAR( (I - LPE) / PPPE) = ADCE

IF $A D C 8>A M A X 8$ TEEN AMAX\& $=A D C 8+1$

IF $A D C 8<$ AMIN\& TEEN AMIN\& = ADC\&

NEXT I

CEOICE:

'PLOT DATA ARRAY ON SCREEN

FOR $I=40$ TO 639

PYE = YOFFS8 - (PLOTAR $(I$ - 40) - AMIN8) / (AMAX8 - AMIN8) *

YMULT:

PSET (I, PY\&)

NEXT I

INPUT "CONTINUE SERIES, SCAN OR EXIT $(X, S, E, P) " ; A S$

IF UCASE\$(AS) = " $Y "$ THEN

$I P G=$ BPE

HPG $=$ HPG $+($ PPP $\star 600)$

CLS 1: GOTO CONVERT

ELSEIF UCASES(AS) = "S" THEN

CAIL SKANSRRN: GOTO CBOICE

ELSEIF UCASE\$(AS) = " $P$ " TEEN

CALI PLOTDATA: GOTO CBOICE

END IF

CIS 1

GOTO ARCBOOSE

CIOSER 1:

SCREEN O: CIS

END SUB

SUB SHIFTLEFT

FOR $I=0$ TO FPTS -1

$\operatorname{ADDAT2}(I)=\operatorname{ADDAT} 1(I)$

$\operatorname{ADDATI}(I)=\operatorname{ADDATO}(I)$

NEXT $I$

INPUT "please entex the full filename": Fspecs: Fs = "Igbasicltorldatal" + FSFEC\$

INPUT "file length (bytes-7)/2 "; FPTSs 


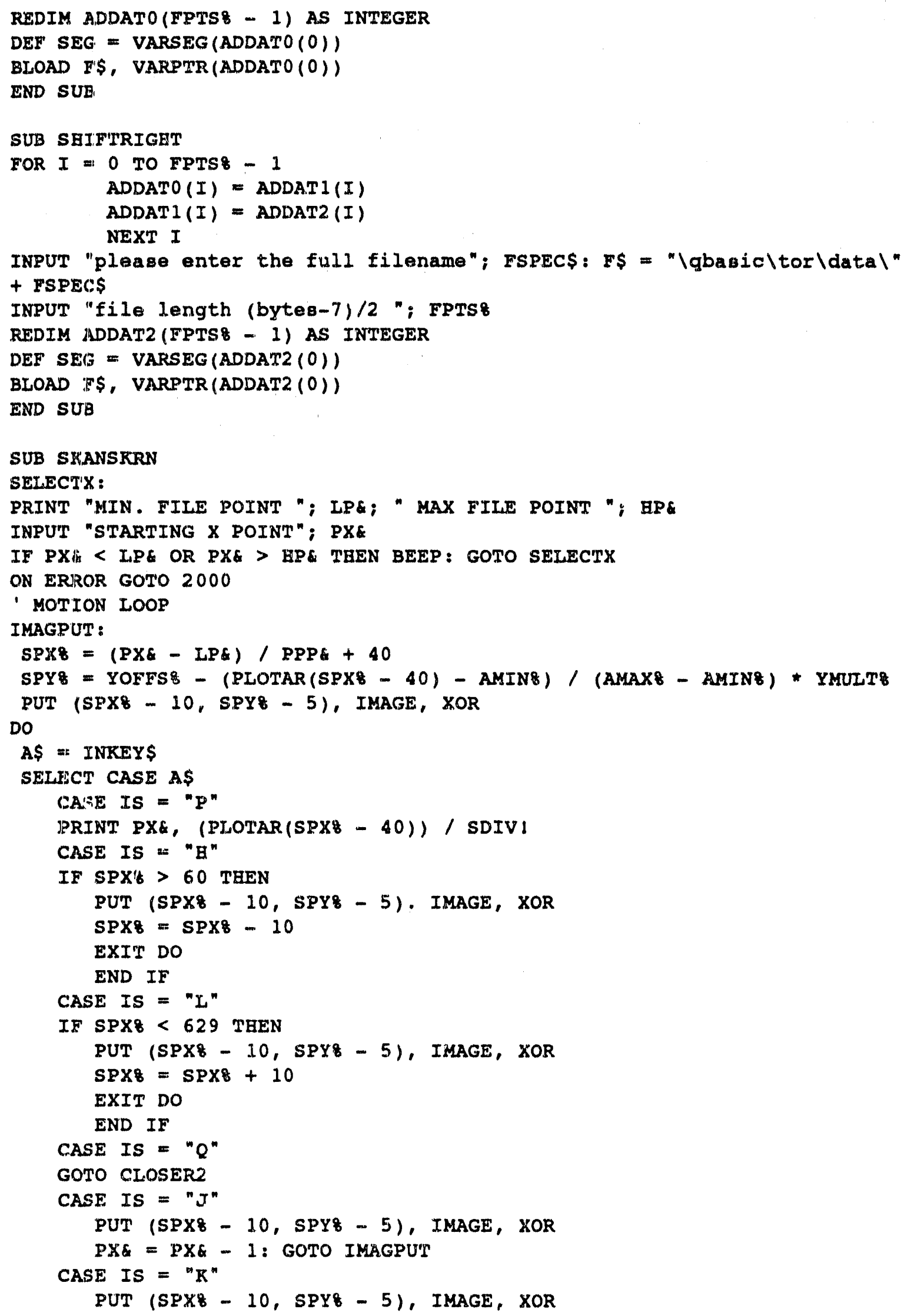




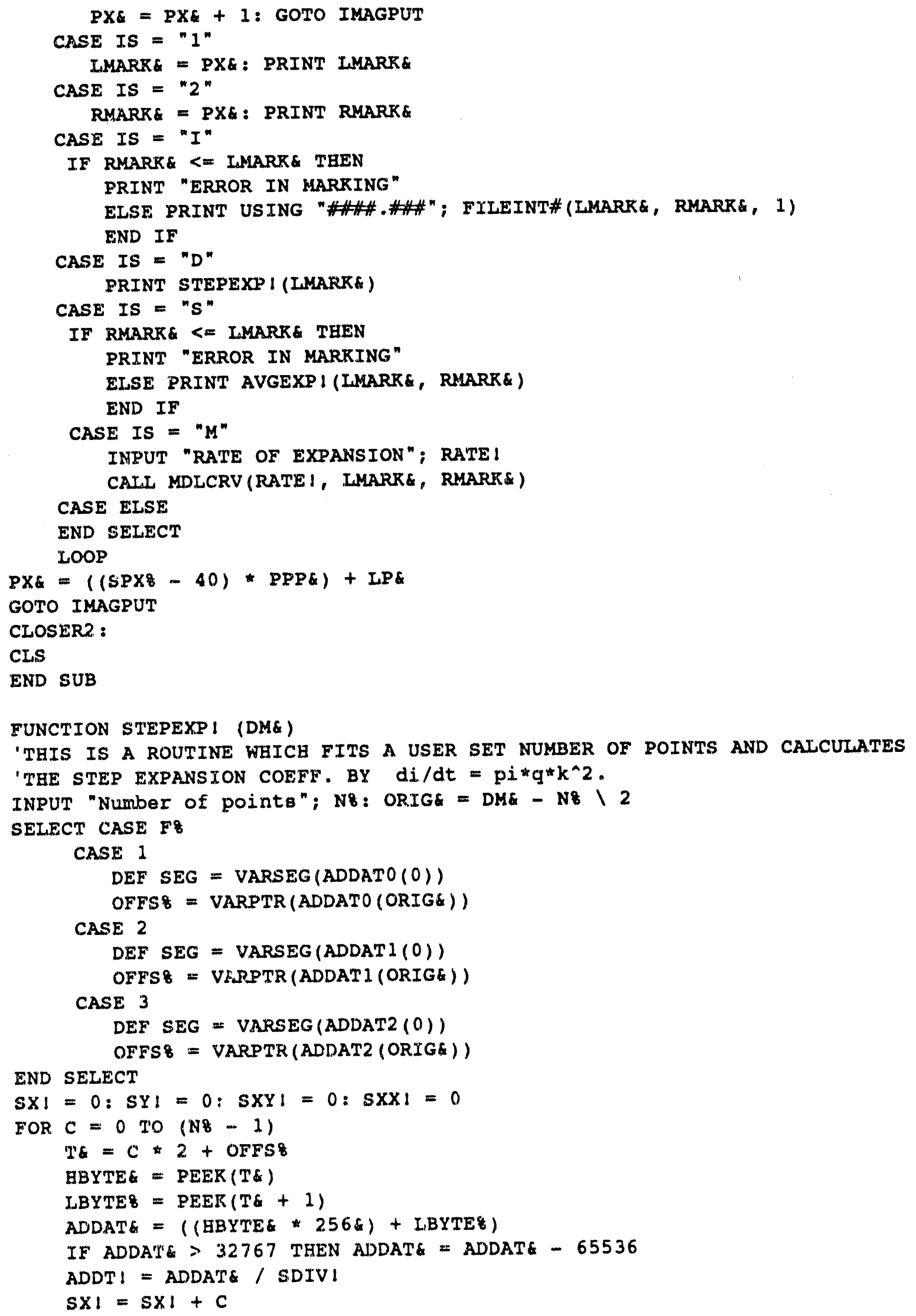




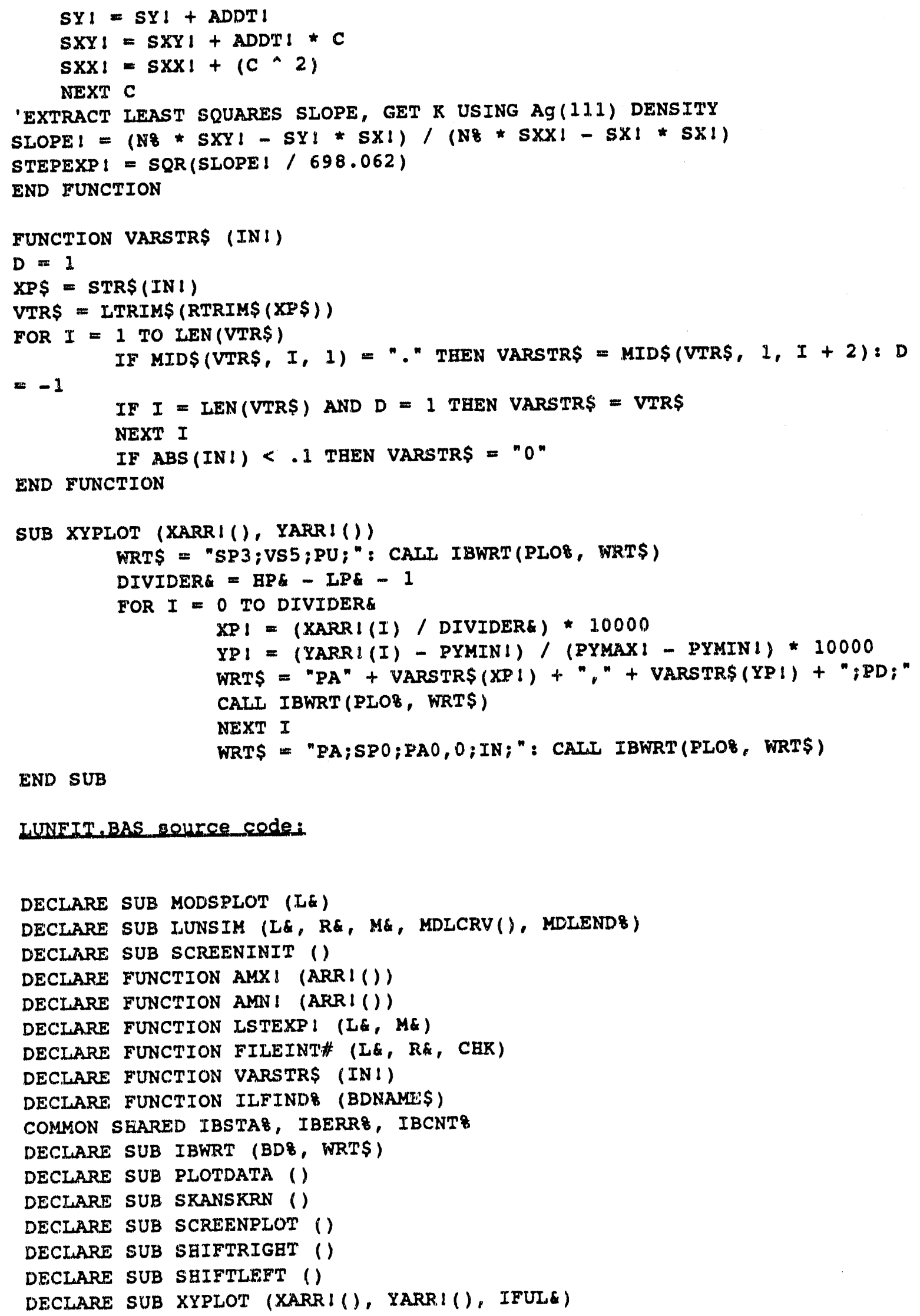




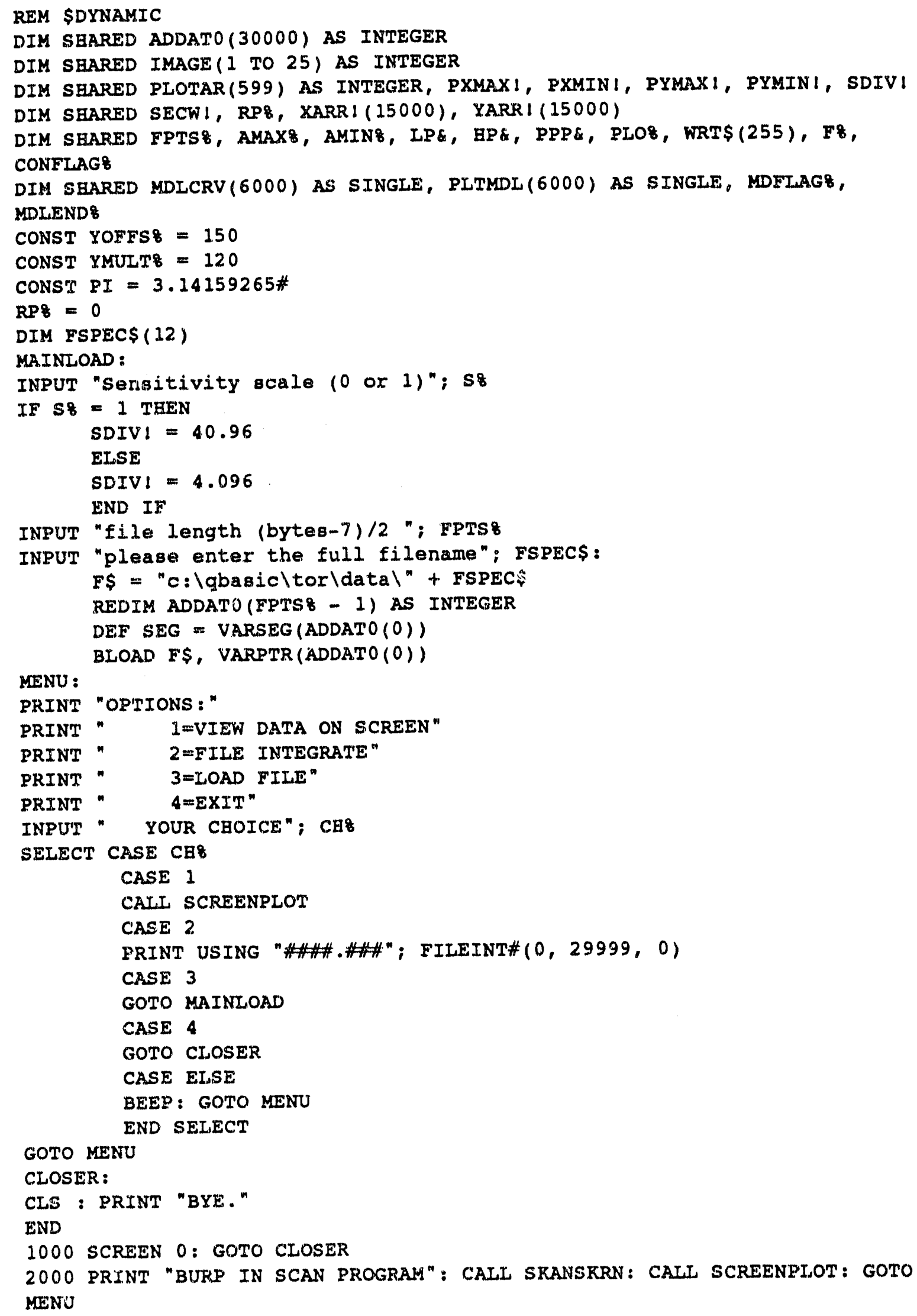




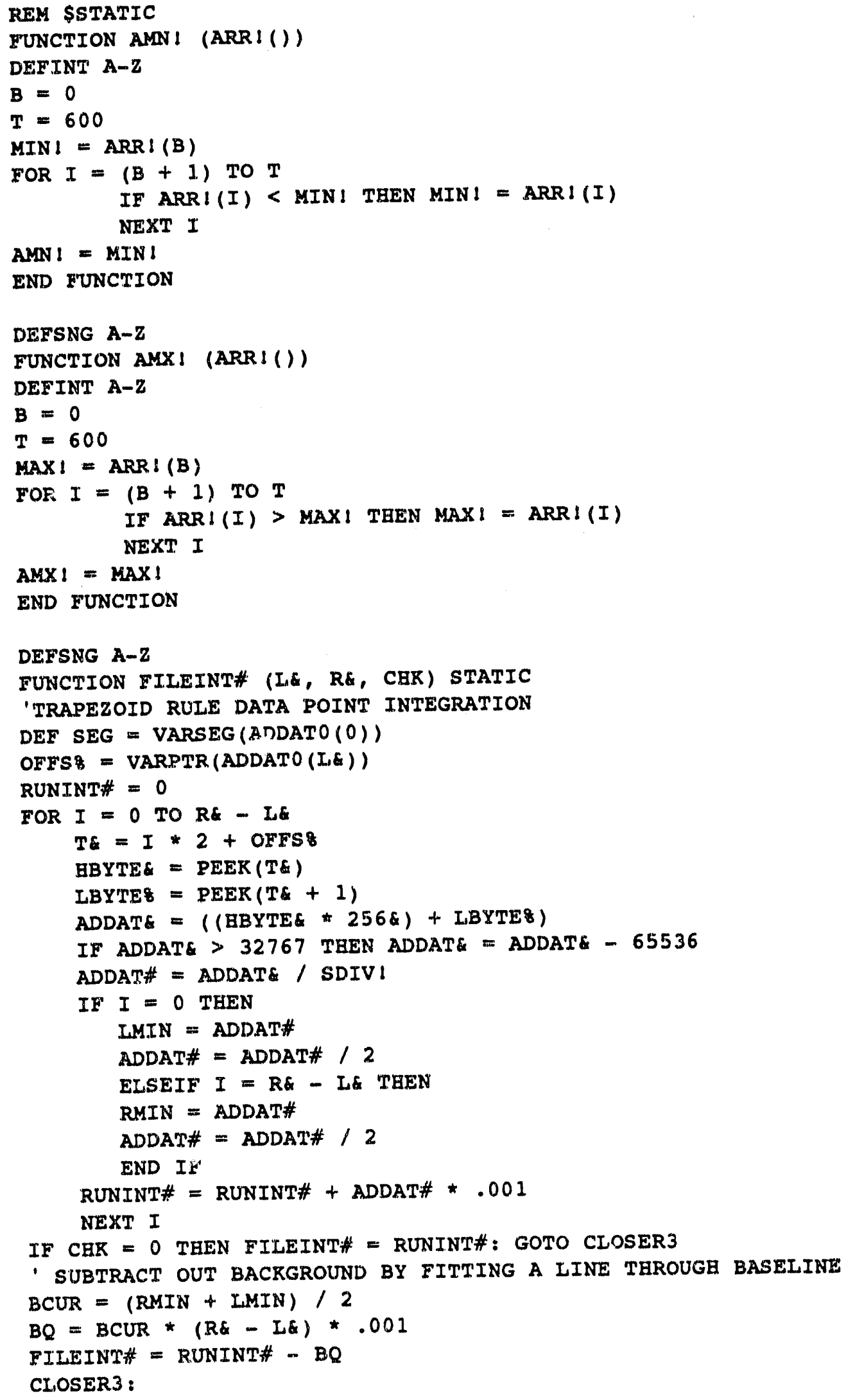


END FUNCTION

FUNCTION LSTEXP! (I\&, M\&)

- THIS IS A ROUTINE WHICH FITS A USER SET NUMBER OF POINTS AND CALCULATES 'THE STEP EXPANSION COEFF. BY $d i / d t=p i * q * k^{\wedge} 2$.

DEF SEG = VARSEG $(A D D A T O(0))$

OFFS $=$ VARPTR (ADDATO (L\&))

$S X 1=0: S Y !=0: S X Y !=0: S X X 1=0$

$\mathrm{N} 8=0$

FOR $C=0$ TO (M\& - L\& - 1)

$T \&=C * 2+$ OFFS

ABYTE\& $=$ PEEK (T\&)

LBYTE\& $=$ PEEK $(T \&+1)$

ADDAT\& $=($ (BBYTE\& * 256\&) + LBYTE\& )

IF ADDAT\& > 32767 THEN ADDAT = ADDATE - 65536

ADDT ! = ADDAT\& / SDIVI

$\mathrm{SXI}=\mathrm{SXI}+\mathrm{C}$

$S Y 1=S Y 1+A D D T !$

$S X Y \&=S X Y 1+A D D T 1 * C$

$\operatorname{sxx} 1=\operatorname{sxx} 1+\left(c^{\wedge} 2\right)$

$\mathrm{NB}=\mathrm{NB}+1$

NEXT $C$

'EXTRACT LEAST SQUARES SLOPE, GET R USING Ag(111) DENSITY

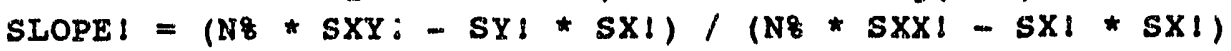

LSTEXP $!=$ SQR (SLOPE $! / 698.062)$

END FUNCTION

SUB MODSRINOT (I\&)

'THIS SUBROUTINE TAKES THE MODEL ARRAY FORM LUNSIM AND PUTS IT INTO

'THE SCREEN BASED PLOTTING ARRAY "PLTMDL"

STARTPTE $=((($ I\& - LP\& $) \backslash$ PPPQ $) * 10)$

$C=0$

FOR I = STARTPTE TO STARTPT\& + MDLEND\& । PPPE

$\operatorname{PLTMDL}(I)=$ MDLCRV $(C)$ * IE+09 * SDIV!

$c=c+$ PPP\&

NEXT I

' PLOT TEE ENTIRE MODEI ARRAY

PY $=$ YOFFS $-(0$ - AMIN\& $) /($ AMAX8 - AMIN\& $)$ * YMULT\&

$\operatorname{PSET}(40$, PYG)

FOR $J=0$ TO 5999 STEP 10

$I=J / 10+40$

PY\& = YOFFS $-($ PLTMDL $(J)$ - AMIN\&) / (AMAX\& - AMIN\&) * YMULT\&

IINE - (I, PY\&)

NEXT J

INPUT "ERASE MODEL CURVE(S)"; B\$

IF UCASE\$(B\$) = "Y" THEN

SCREEN 0

CALI SCREENINIT

FOR I $=40$ TO 639

PY\& = YOFFSZ - (PLOTAR(I - 40) - AMINZ) $/($ AMAXZ - AMINZ) *

YMUL'T웅

PSET (I, PY\&)

NEXT I 


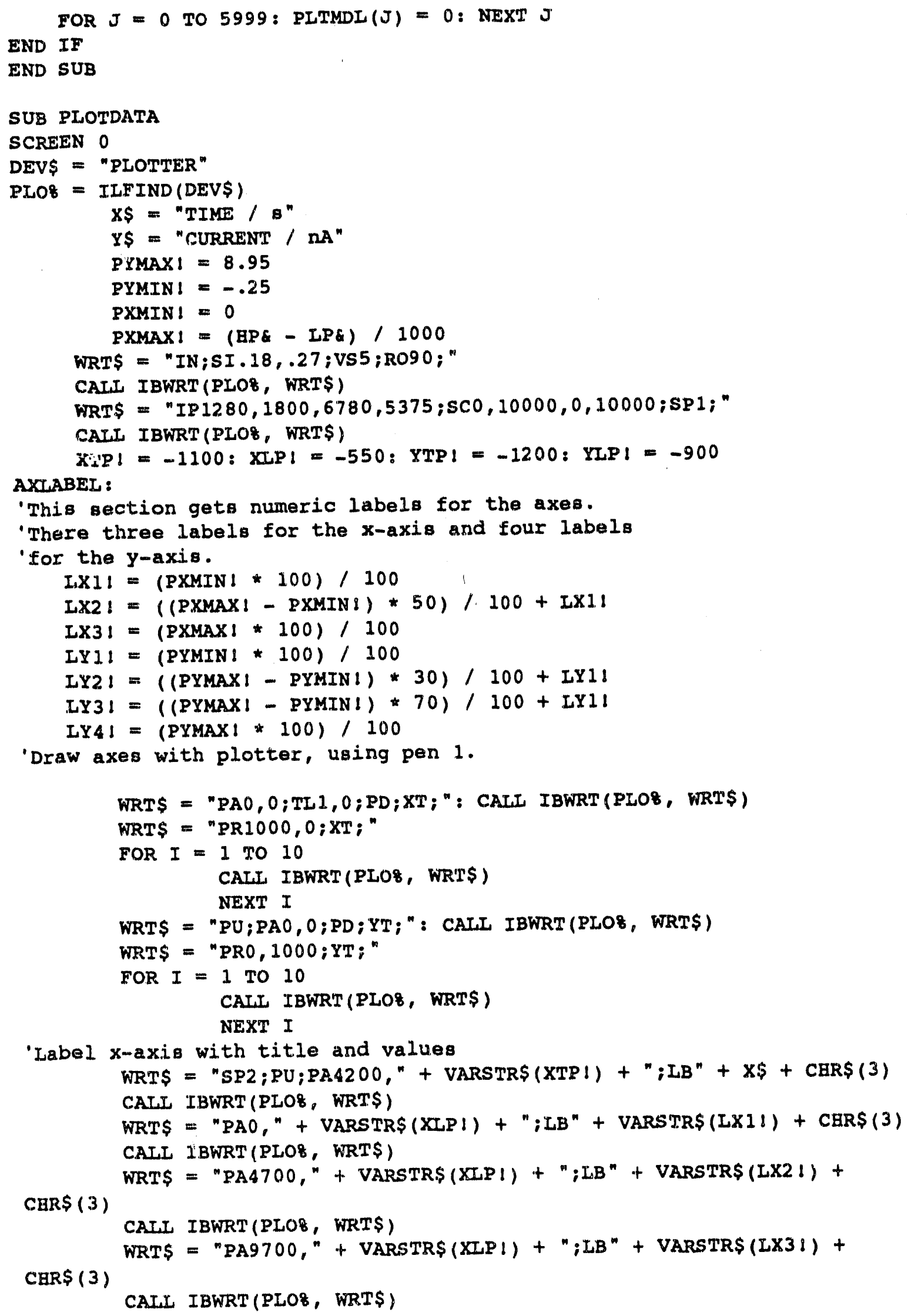




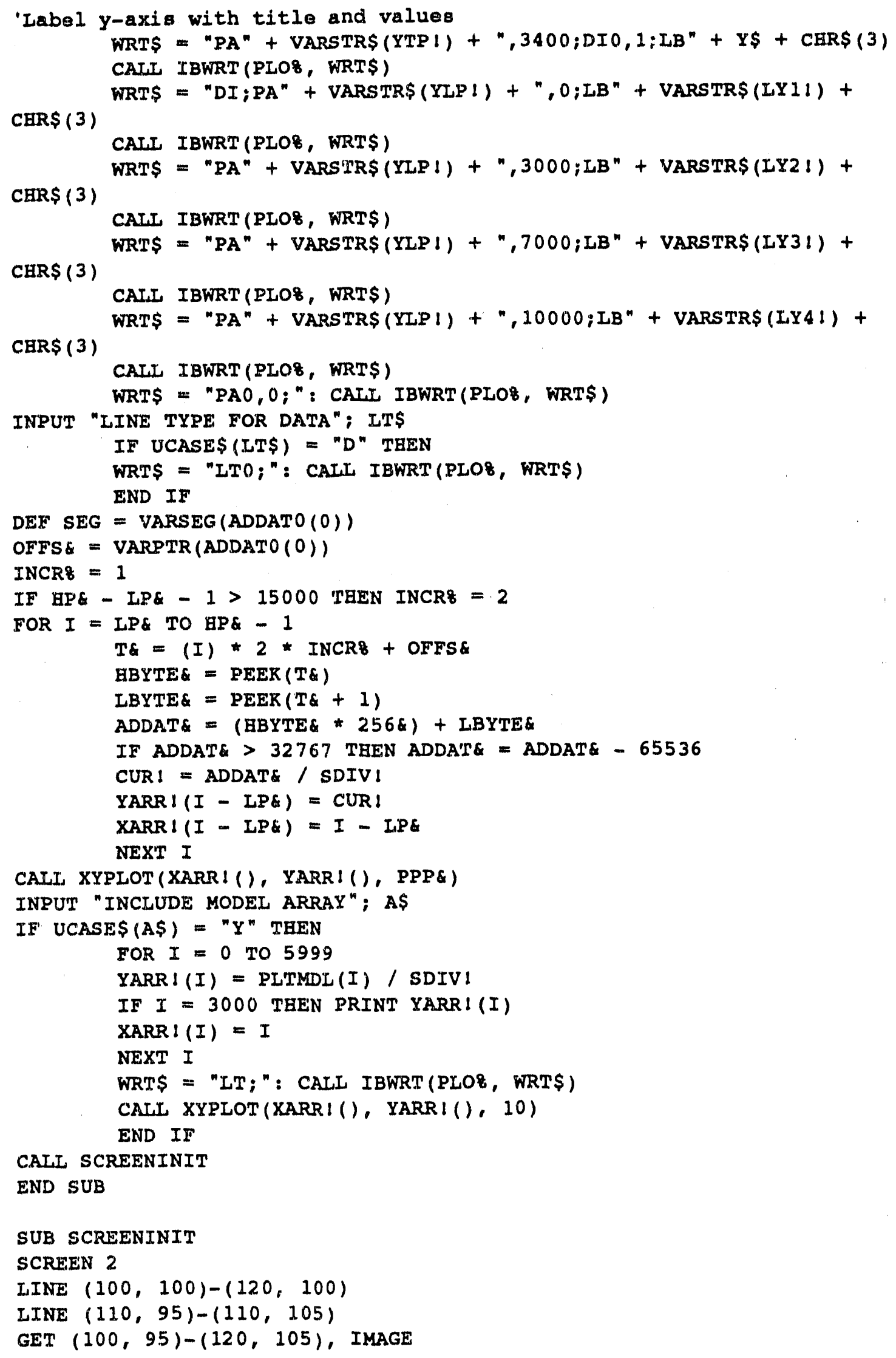


PUT $(100,95)$, IMAGE, XOR

VIEW SCREEN $(40,0)-(639,167), 1$

VIEW PRINT 23 TO 24

END SUB

SUB SCREENPLOT

ARCBOOSE :

INPUT "PLOT FILE (999) TO EXIT"; F\&

SELE:

SEINECT CASE FE

CASE 1

DEF SEG = VARSEG $(\operatorname{ADDATO}(0))$

OFFSE $=\operatorname{VARPTR}(A D D A T O(0))$

CASE 999

GOTO CLOSERI

CASE ELSE

INPUT "SELECT FILE 1 OR 999 TO EXIT"; F\&: GOTO SELE

END SELECT

INPUT "POINTS PER PIXEL"; PPP\&

INPUT "STARTING POINT"; SP\&

LPG $=$ SPQ - I

BP\& $=$ LP\& $+($ PPPE *600)

IF GP\& > FPTS 8 - 1 TEEN BP\& = TRTS -1

CONVERT:

$R P Z=0$

PPPE $=($ BP\& - LP\& $) / 600$

$\operatorname{AMAXZ}=1$

AMINO $=5000$

FOR I = LP\& TO (BP\& - 1) STEP PPP\&

$T \&=2 * I+$ OFFS \&

BBYTE\& $=$ PEER (T\&)

LBYTE $=\operatorname{PEER}(T \&+1)$

ADDATE $=($ BBYTE\& * 256) + LBYTE\&

IF ADDAT\& > 32767 THEN ADDAT\& = ADDAT\& - 65536

$A D C 8=\operatorname{CINT}(A D D A T \&)$

PLOTAR ( I - LPE) / PPP\&) = ADC\&

IF $A D C 8>$ AMPXO TEEN AMAXZ $=A D C 8+1$

IF ADC8 < AMINO THEN AMINZ = ADC8

NEXT I

CHOICE :

'PLOT DATA ARRAY ON SCREEN

CALI SCREENINIT

IF RPQ = 1 THEN GOTO CONVERT

FOR $I=40$ TO 639

PY\& = YOFFSZ - (PLOTAR $(I-40)-$ AMIN8) $/($ AMAX8 - AMIN8 $)$ *

YMULTR

PSET (I, PY\&)

NEXT I

INPUT "CONTINUE SERIES, SCAN OR EXIT (A,Y,S,E,P)"; AS

IF UCASE $\$(A \$)=$ " $Y$ " THEN

$I P \&=B P \&$

$\mathrm{BP \&}=\mathrm{BP \&}+(\mathrm{PPP} \& * 600)$

IF BPG > 29999 THEN PRINT "OUT OF RANGE": GOTO CHOICE 


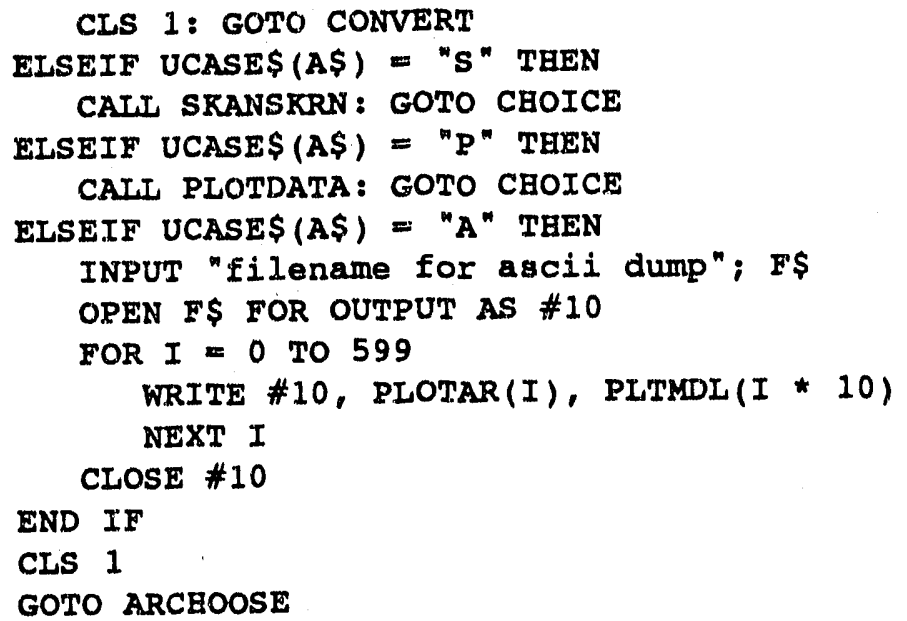




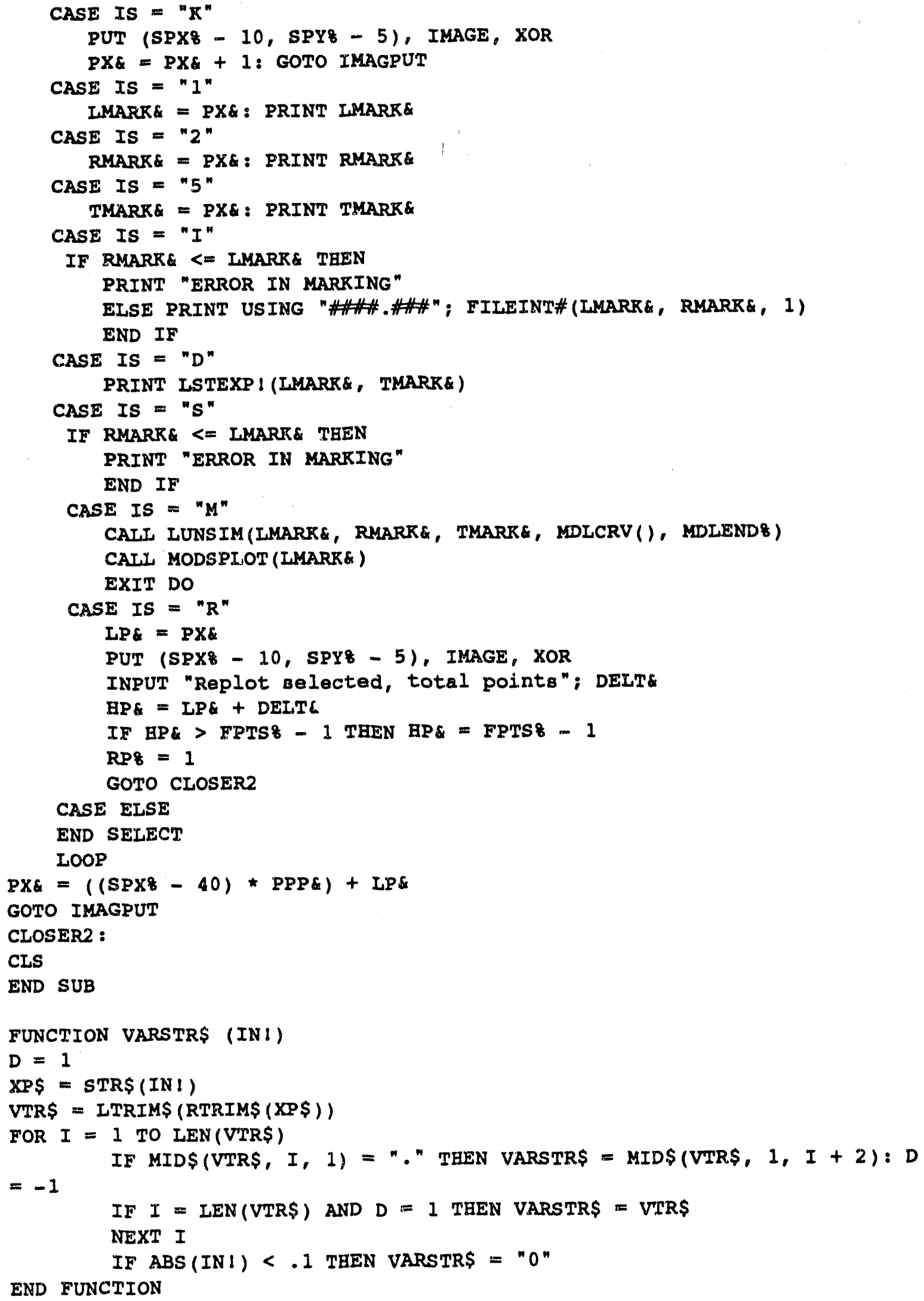


SUB XYPLOT (XARR! (), YARRI(), IFUL\&)

WRT\$ = "SP $3 ;$ VS5;PU;": CAIL IBWRT (PLO8, WRT\$)

DIVIDERE $=($ BP\& - IP\& - 1) * (IFUL\& / PPP\& $)$

FOR $I=0$ TO DIVIDERE

$X P \mid=(X A R R !(I) /$ DIVIDERA $) * 10000$

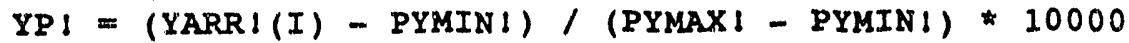

WRT $\$=" P A "+\operatorname{VARSTR} \$(X P 1)+"$ " + VARSTR\$(YPI) + ";PD;

CALI IBWRT (PLOZ, WRT\$)

NEXT I

END SUB

WRT\$ = "PA;SPO;PAO , ; " : CAIJ IBWRT(PLO8, WRT\$)

Code for IUNSIM module of IUNEIT.BAS:

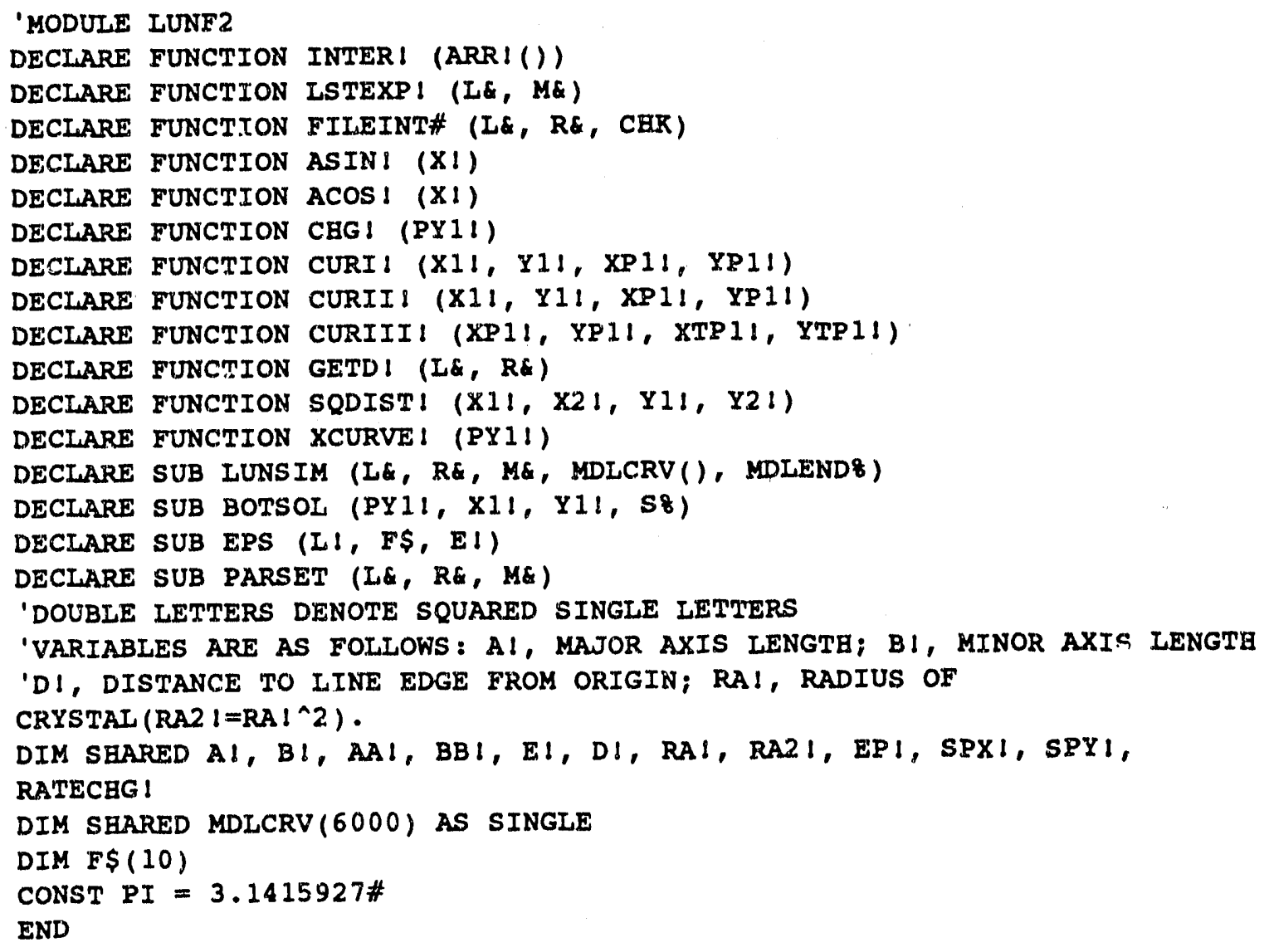




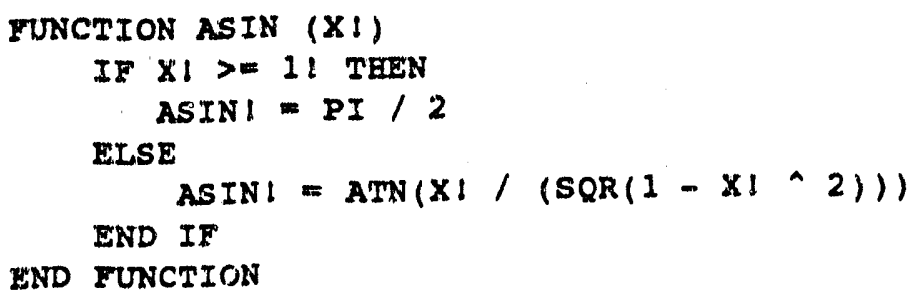




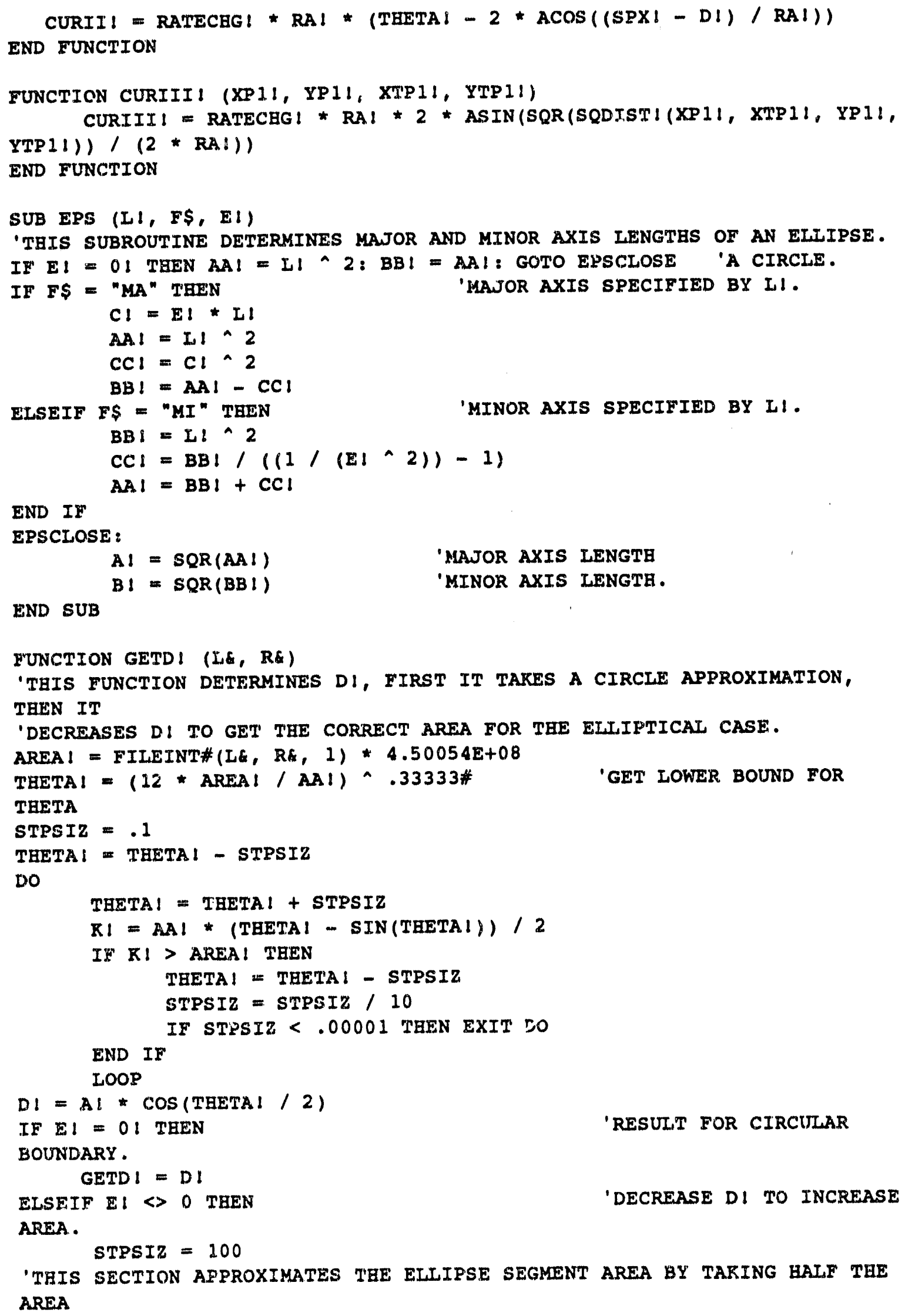


'AND SUBTRACTING TEE AREA OF A TRAPEZOID AND TWO SMALI APPROX. CIRCULLAR 'SEGMENTS. THE TRAPEZOID AREA CEANGES WITH DI, BUT CIRC. SEGS ARE ASSUMED NOT

'TO CHANGE DRAMATICATIY WITH A SHALL DI CHANGE. THIS WILL PRODUCE SIGNIFICANT

'ERROR FOR LARGE EI.

DO

$D !=0 !-S T P S I Z$

SECTEl $=P I / 2-A \operatorname{Cos}(D / / A l)$

SECAR! = AA! * (SECTH! - SIN(SECTB!))

SECAR!

$R !=P I / 2 * A l * B l-(1+\operatorname{SQR}(1-(D) * 2) / A A l)) * B ! * D l-$

IF $K$ ! > AREA! THEN

$D I=D I+$ STPSIZ

SIPSIZ $=$ STPSIZ / 10

END IF

IF STPSIZ $<.01$ THEN EXIT DO

LOOP

GETD 1 = DI APPROX. RESULT FOR

ELIIP. BND.

END IF

END FUNCTION

FUNCTION INTER! (ARR!())

SUMI $=0$

YOR I $=0$ TO 600

SUM! $=$ SUM! + ARR! I $) * .001$

NEXT I

INTERI $=$ SUMI

END FUNCTION

SUB LUNSIM (L\&, R\&, M\&, MDLCRV (), MDLEND\&)

FLAG8 $=0$

START:

CALE PARSET(LE, RE, ME)

INPUT "OFFSET FROM MAX IN UNITS OF nM "; OPXI: SPX! = Al - OPX!

SPY I $=\operatorname{SQR}(B B \mid *(1-\operatorname{SPX}|\wedge 2 / A A|))$

PYI1 = SPY 1: PYP 11 = SPY 1: EP! $=1000$

$\operatorname{MDLCRV}(0)=01$

$\mathrm{X} 11=0: \mathrm{Y} 11=0: \mathrm{XP} 11=0: \mathrm{YP} 11=0$

$B !=A !-D !$

'COMPUTE SPACING IN nM PER $0.1 \mathrm{~ms}$ POINT USING INPUT EXPANSION RATE

SCALE ! = LSTEXP! (L\&, MQ) * 10001

INPUT "NUMBER OF SIMULATION POINTS IS SELECTED BERE"; FUL\&

FOR $I=0$ TO 5999 STEP FUL\&

$R A 1=I *$ SCALE!

$R A 2$ ! = RAI ^ 2

IF RA! < = B! - OPX! THEN 'CURRENT REGIEME 1

CALL BOTSOL (PY11, X11, Y11, 1) 'GET INC Y SOLUTION

CALI, BOTSOL (PYP11, XP11, YP11, -1)'GET DEC Y SOLUTION

$\operatorname{MDLCRV}(I)=\operatorname{CURI} 1(X 11, \mathrm{X} 11, \mathrm{XP} 11, \mathrm{YP} 11)$

PY11 = Y11: PYP 11 = YP 11

ELSEIF RAI > BI - OPXI THEN

'INTERACTING WITE LINE ALSO 


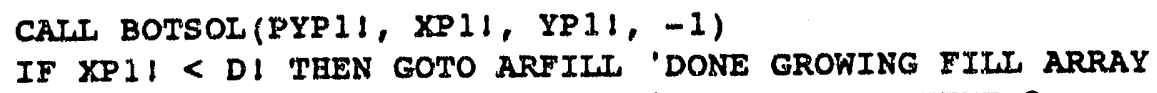




\section{APPENDIX B}

Derivation of equation 2 chapter 1:

$\square$ Consider growth of a new phase 3 from a supersaturated phase 2 onto a substrate phase 1;

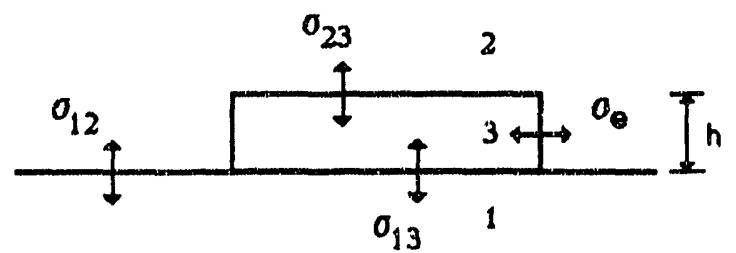

relevant surface tensions $\sigma_{12}, \sigma_{23}, \sigma_{13}$ and $\sigma_{e}$ are as labeled.

The new phase consiots of m particles with "atomic" volume v. The formation of the new phase is an activated process which goes through a critical cluster containing $\mathrm{m}^{\star}$ particles. A balance of free energies determines $\mathrm{m}^{\star}$.

a The total free energy for overpotentials, $\eta$, with respect to the equilibrium potential to form a cluster of m particles of like upon like is a sum of volume, surface, edge, and entropy terms;

$$
\Delta G(m)=(m v)\left[\frac{-z e \eta}{v}\right]+\left(\frac{m v}{h}\right) \sigma_{13}+2(m \pi v h)^{1 / 2} \sigma_{e}+G_{C}
$$

$G_{c}$ is the surface precursor entropy term. For like upon like, $o_{12}$ and $\sigma_{23}$ terms do not appear, $\sigma_{13}$ is kept to account for stabilization of the 
new phase by a defect at the nucleation site. The use of the overpotential to describe the free energy for volume formation reduces the need for inclusion of ionic solvation $108 \mathrm{~s}$ and other issues which control the equilibrium potential. The entropy term has been derived by Lothe and Pound to be $G_{C}=k \ln \left(\Gamma_{A g^{O}}\right)$, however, this term is a source of controveray. For this derivation, the functional details of $\mathrm{G}_{C}$ are less important, as long as it is independent of $\mathrm{m}$.

$\square$ Differentiating $\Delta G(m)$ by $m$ and solving for the critical cluster size by setting

$$
\left(\frac{\delta \Delta G(m)}{\delta m}\right)=0
$$

gives:

$$
m^{*}=\frac{(\pi v h) \sigma_{e}^{2}}{\left[z e \eta-\frac{v}{h} \sigma_{13}\right]^{2}}
$$

$\square$ substituting $\Delta G\left(\mathrm{~m}^{*}\right)=\Delta \mathrm{G}^{+}$;

$$
\Delta G^{t}=\frac{(\pi v h) \sigma_{e}^{2}}{\left[z e \eta-\frac{v}{h} \sigma_{13}\right]}+G_{c}
$$

Drate of nucleation $A$, assuming $\Delta G^{\dagger}$ is the activation energy;

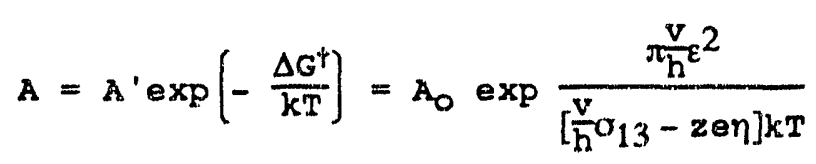


to get the final result, we have substituted a one dimonsionsi edge energy $\varepsilon=\sigma_{e} h$, for the "edge" tension. 
ASYST code to generate model curves in sets of 100 . For ASYST users, the loading order is first RSIM4.TXT, then CrNS.TXT.

CRNS. TXT (short for "create-n-gave"):

I THIS TEXT, WAEN LOADED AFTER RSIM4.TXT OR EQUIVALENT LULLOWS FOR THE $\backslash$ GENERATION OF MULTIPLE CURRENT TIME TRANSIENTS FROM RANDOM POINTS ( WITHIN AN ELLIPTICAL ELECTRODE. RUNNING PROCEDURE IS AS FOILOWS:

(1) LOAD RSIM4.TXT (OR EQUIVALENT)

(1) LOAD TEIS FILE

(3) RUN TEIS FILE BY EXECUTING CREATE.N.SAVE

I THIS PROGRAM NOMINALIY GENERATES AND STORES 100 TRANSIENTS AND THEIR 1 LOCATIONS IN A USER SPECIFIED FILE

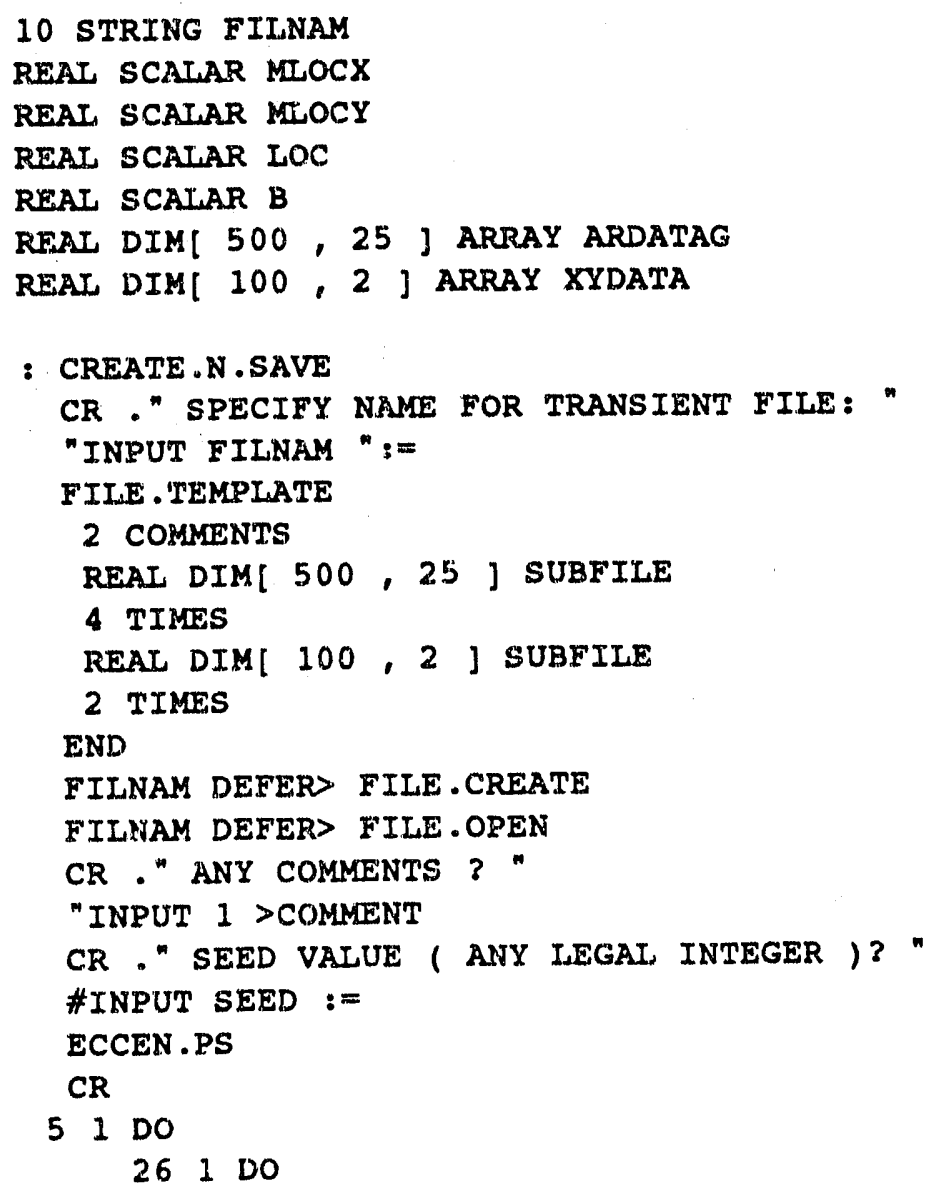




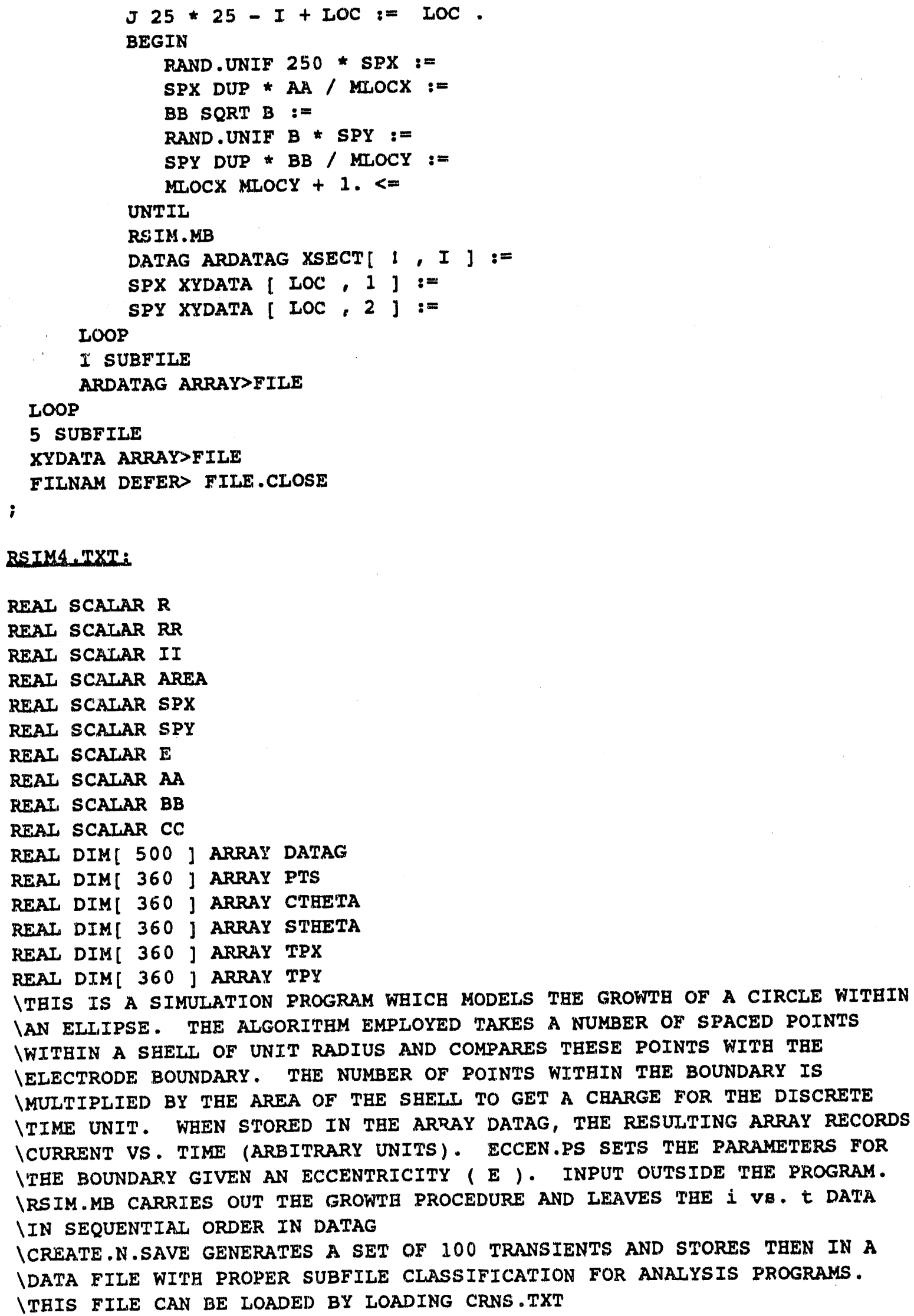

RSTMA TXT: 


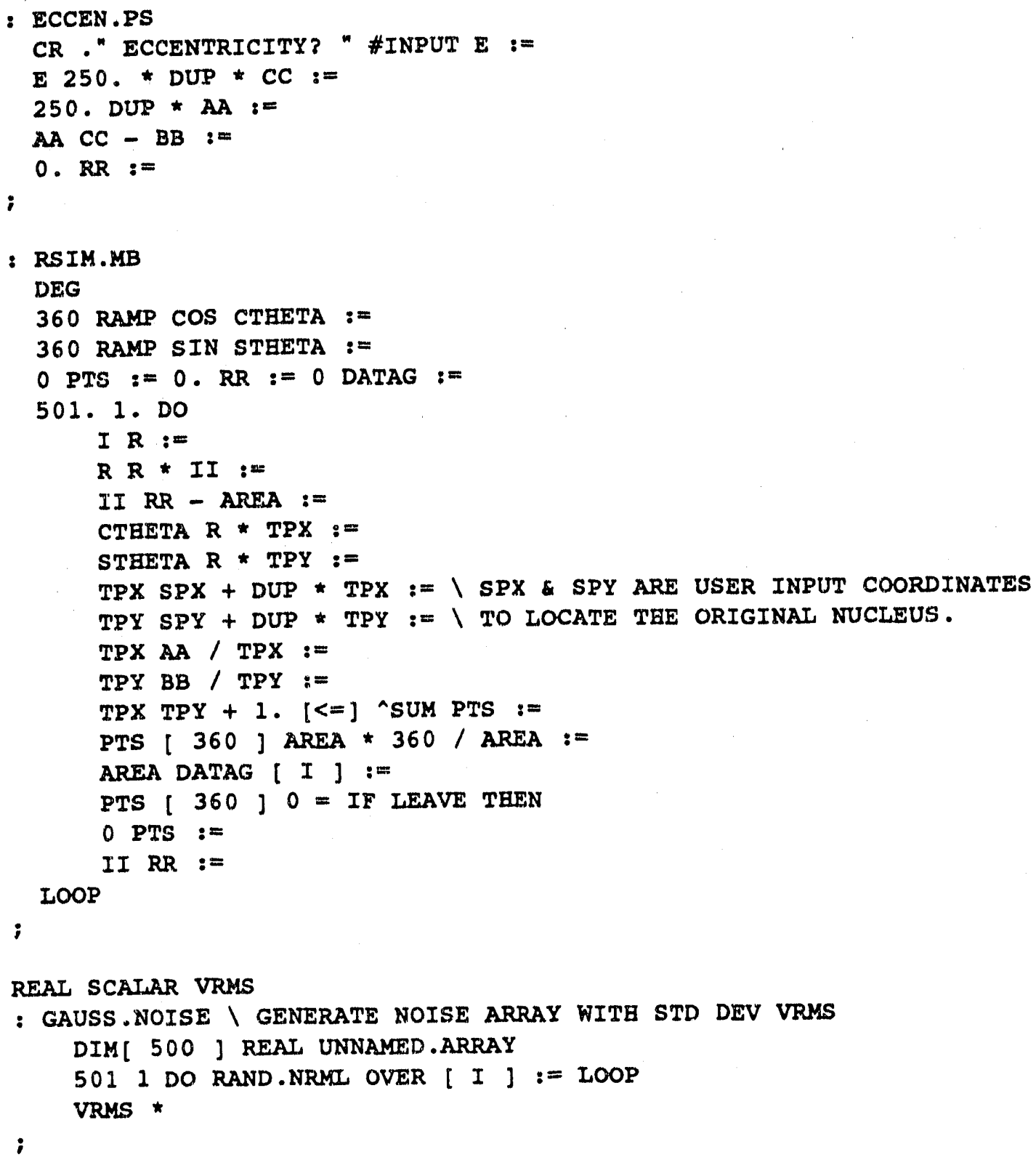

Code which demongtrates a way of extracting critical points from simulated data, RROTM.TXT:

। THIS FILE WILL PROCESS SIMULATED TRANSIENTS GENERATED BY CRNS.TXT OR 1 EQUIVALENT. AII VARIABLES NEEDED ARE DECLARED WITEIN THIS TEXT.

ITI AND T2 ARE STORED IN ORDER IN SUBFILE 5 OF DATA FILE.

$1 \mathrm{~T} 1$ IS DERIVED FROM THE FIRST DEVIATION EROM LINEAR BEEAVIOR.

1 T2 IS DERIVED BY A FIT THROUGB TAE END OF THE CURVE. 


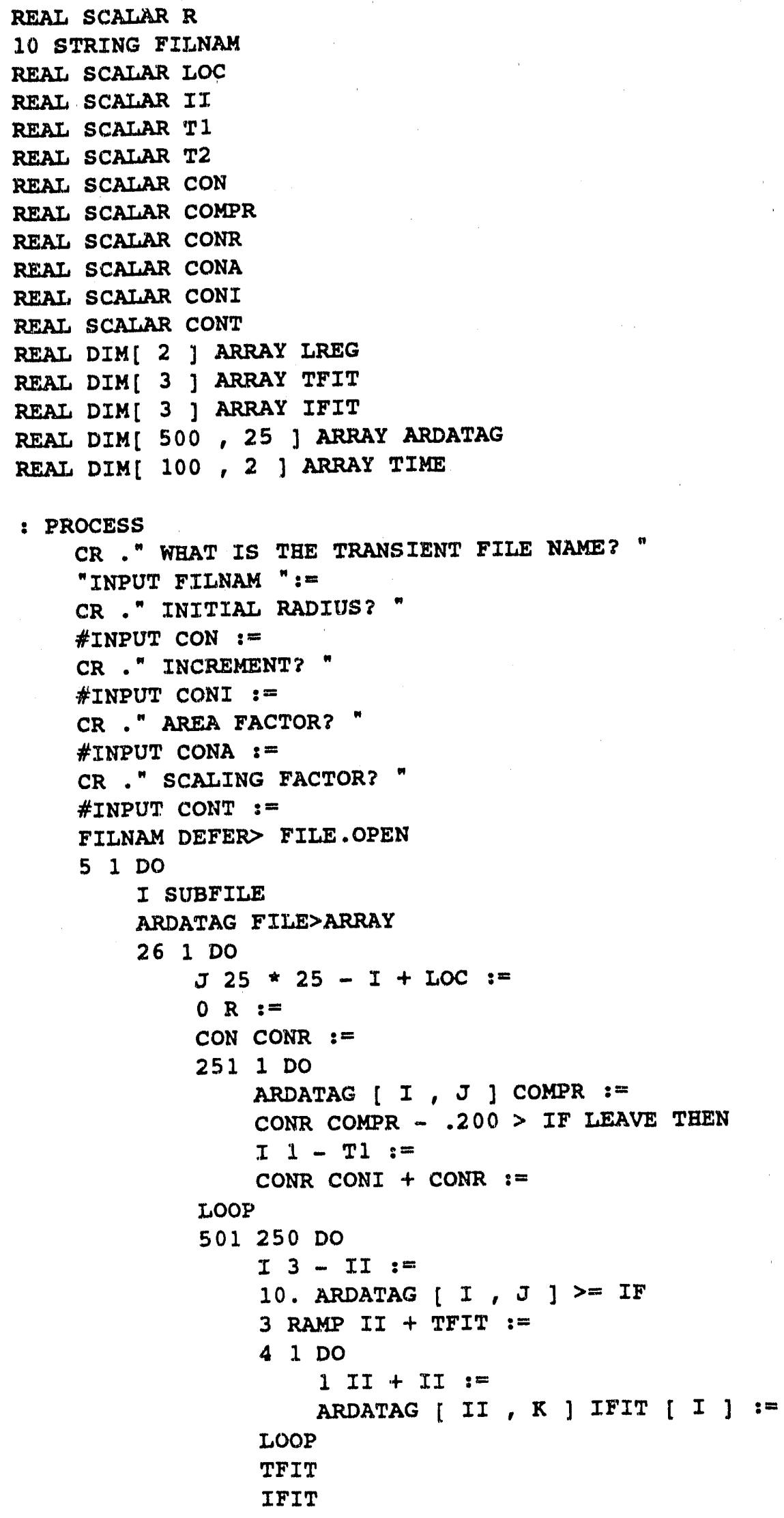




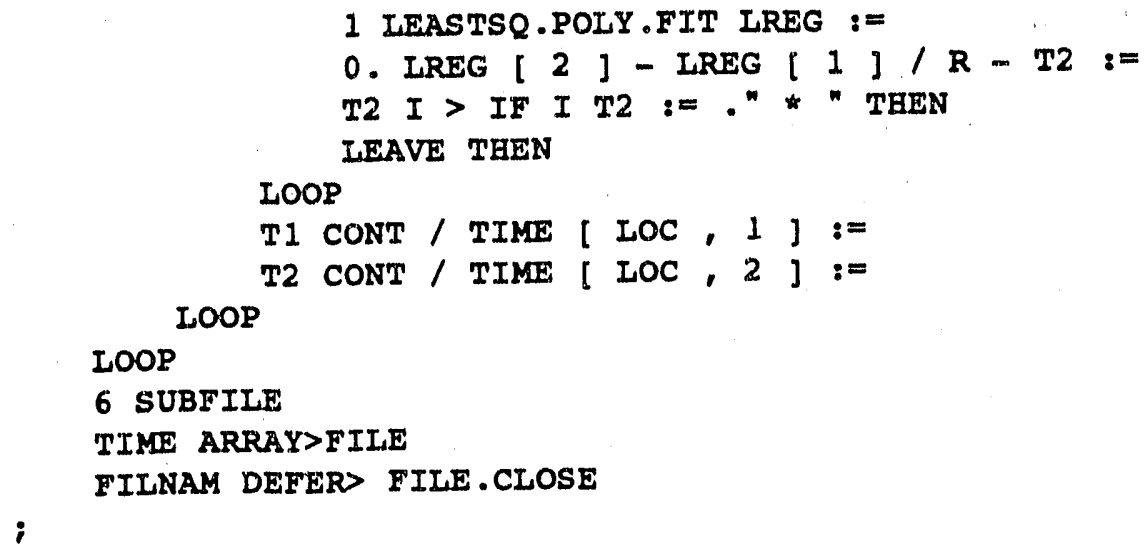

3) CALI XYCONV2 WHICB WILL CONVERT TEE TIMES INTO COORDINATES

ITEE ALGORITHM USED DEFINES AN ELIIPSE UPON WHICA THE NUCLEUS LIES BY ISUBTRACTING THE FIRST TIME( IN RADIAL UNITS ) FROM THE MAJOR AND MINOR \AXES. THEN, THE NORMAL TO THE ORIGINAL ELLIPSE OF LENGTH T2 WHICA LIES ION THE CALCULATED ELIIPSE IS DETERMINED. THIS POINT OF INTERSECTION IS ITHE LOCATION OF THE INITIAL NUCLEUS.

REAI SCALAR R

REAI, SCALAR E

REAL SCAIAAR AA

REAL SCALAR BB

REAL SCATAAR CC

REAL SCALAR A

REAL SCALAR B

REAL SCATAR C

REAI SCAIAAR PX

REAL SCALAR PY

REAL SCALAR R1

REAL SCALAR T2

PEAL SCALAR XPARA

REAC SCALAR YPARA

REAI SCALAR AAP

REAL SCALAR BBP

REALI SCALAR P

REAL SCALAAR THETA

REAL DIM[ 100 ] ARRAY APX

REAL DIH[ 100 ] ARRAY APY

REAL DIM[ 100 ] ARRAY RRI

REAI DIM[ 100 ] ARRAY RR2 


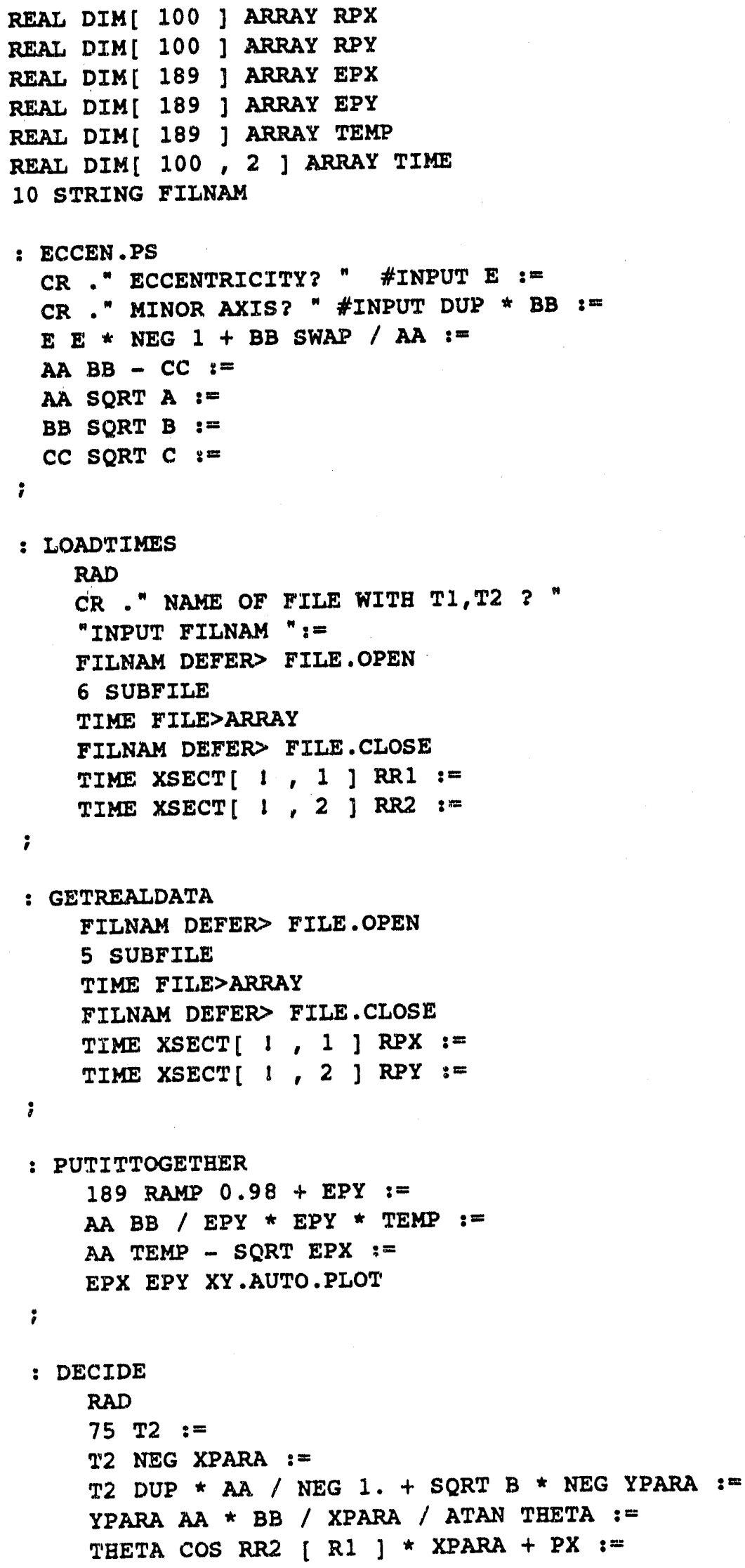




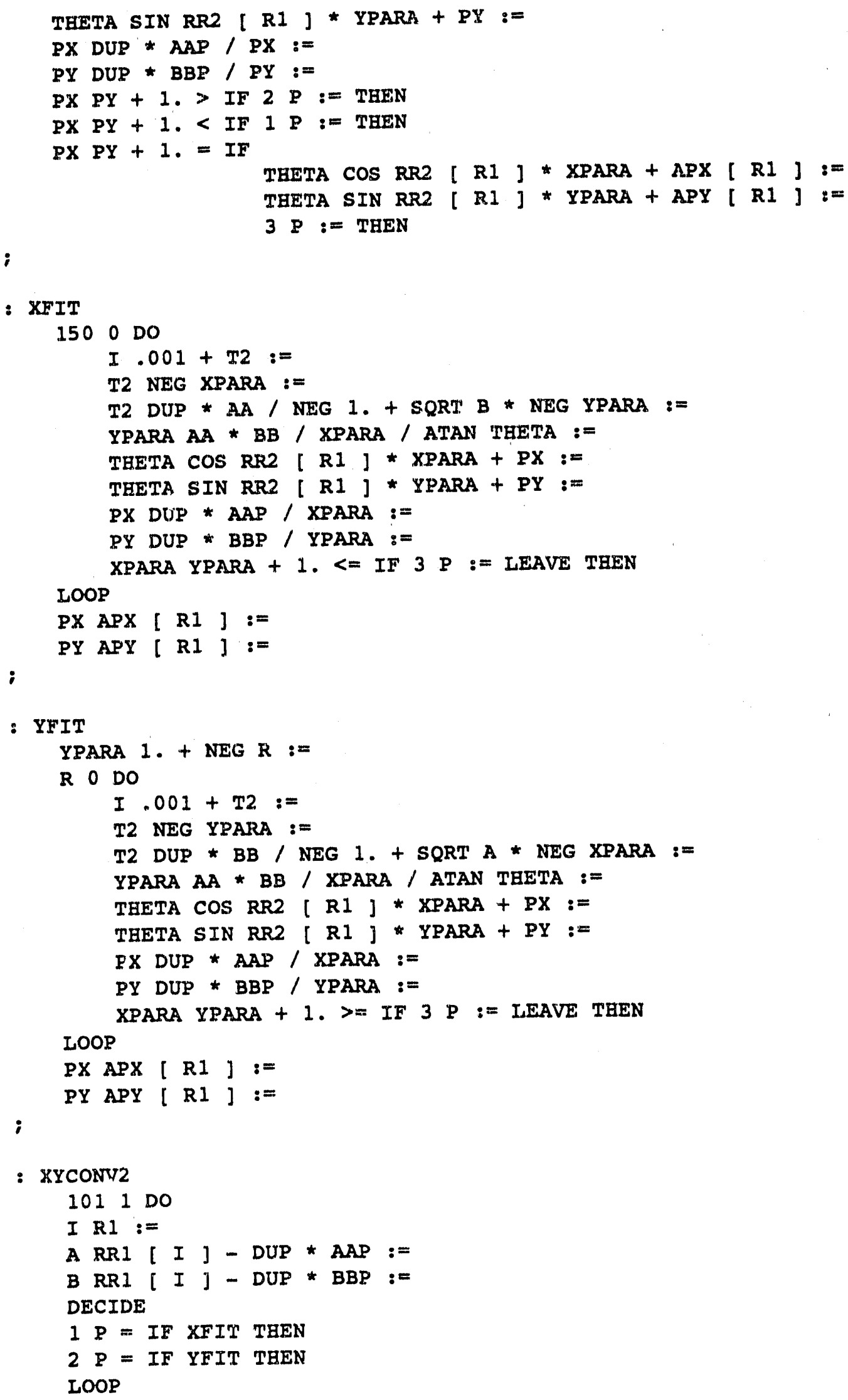


CR ." DONE. "

; 


\section{APPENDIX D}

scanning tunneling microscope electronics schematics. There axe a total of 4 circuit cards depicted here. The content of the third card is split into two figures here for clarity. This division is also a logical one because the functionality of board 3 part $\mathrm{B}$ will be incorporated into a fifth card which will allow plug-in changes to the feedback mechanism. Captions for the figures will precede the pages which contain the figures themselves.

Figure DI: I'his circuit amplifies and buffers digital oignals from the computer for use in the experiment. The majority of the output lines are used for switching relays elsewhere within the electronica; however, the control signal for the sample-and-hold circuitry is generated here as well. The outpute from the computer interface card, the Metrabyte DDA-06, are not etrong enough to drive typical reed relays. High-gain Darlington transistors $2 \mathrm{~N} 6039$ amplify the current signal from the DDA-06 digital signals to sutable levels. At this revision, there is an excess of drive lines with respect to the total number used, this allows painless expansion to include additional digital controls at a later date. 


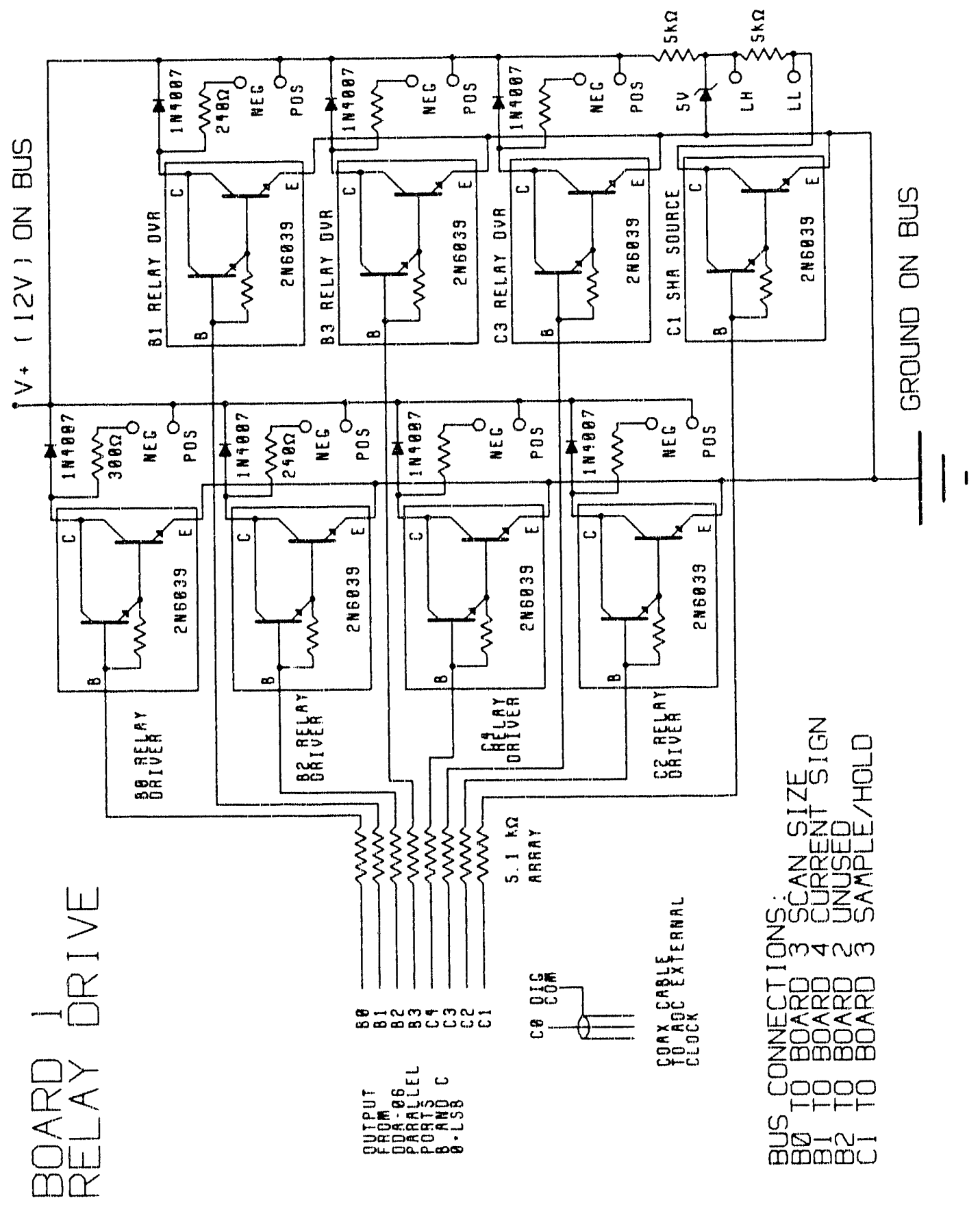


Figure D2: These three circuit modules all reside on the same card. One purpose of the card is to provide analog control signals to the experiment in the form of coarse PZT control and sample bias. Coarse PzT control signals are generated by an active 2-pole Bessel filter with a 10 second rise-time. The sample bias is generated by adding the control signal from the computer and the signal from the lock-in amplifier. This circuit is designed to begin rolling off oignals at $12 \mathrm{kBz}$. The narrow bandwidth here reduces total noise throughput to the experiment, but also limits the available window for spectroscopic measurements. The other function of this card is to provide a module for subtracting off a constant portion of the vertical control signal. The difference between the control signal and the input analog aignal. from the computer is multiplied by ten, allowing sensitivity of $1 \mathrm{~nm} / \mathrm{V}$. This quantity translates into $5 \mathrm{pm}$ per bit after the analog-to-digital conversion. 


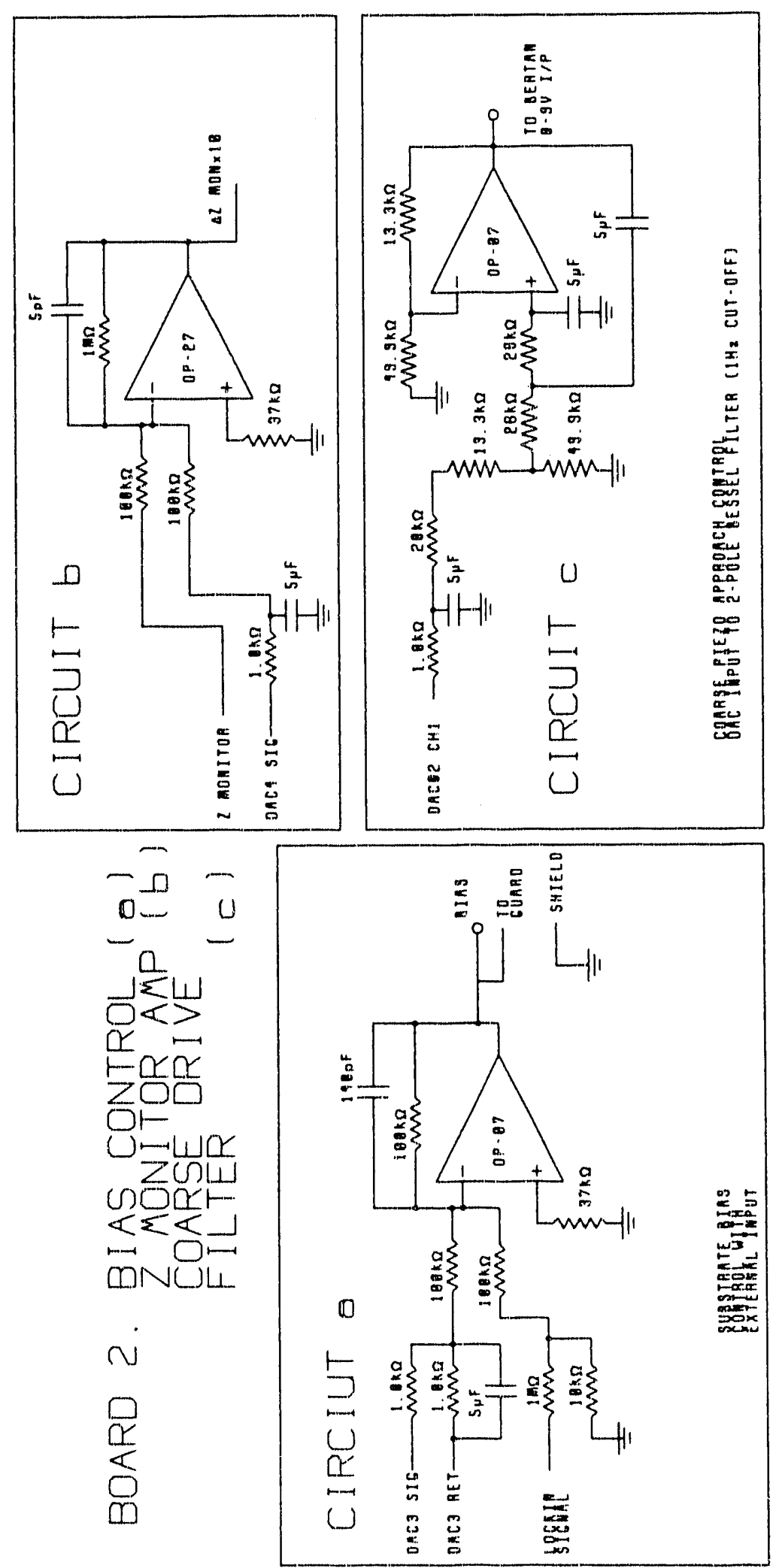


Figure D3: This circuit is the first part of the total circuit on board 3. This part takes analog inputs from the computer via the DDA-06 interface card and converts them into the drive signale for the scanning piezoelectric elements. Scan signals in $x$ and $y$ directions are scaled and multiplied by -1 in the first stage of the analog computer, on the upper left of the figure. The scaled signals and their complements are combined with the vertical control signal in the three summing amplifiers (OP-27 operational amplifiers). Each suming amplifier sends output to its own high-voltage amplifier which scales the signal 25 times. Since piezoelectric elements contract with increasing voltage, the most positive signal to the elements comes from the power supply, and the "return" comes from the output of the high-voltage amplifier. With this configuration, the maximum signal corresponde to almost zero voltage applied across the element, resulting in maximum extension. The dual-amplifier circuit at the bottom of this figuxe is an active 4-pole Bessel filter with a rise-time of 1 second. This filter is to block computer noise on the gignal which determines the tunneling current feedback level. The 4-pole Bessel filter provides very steep roll-off compared to RC filters and is very effective. 


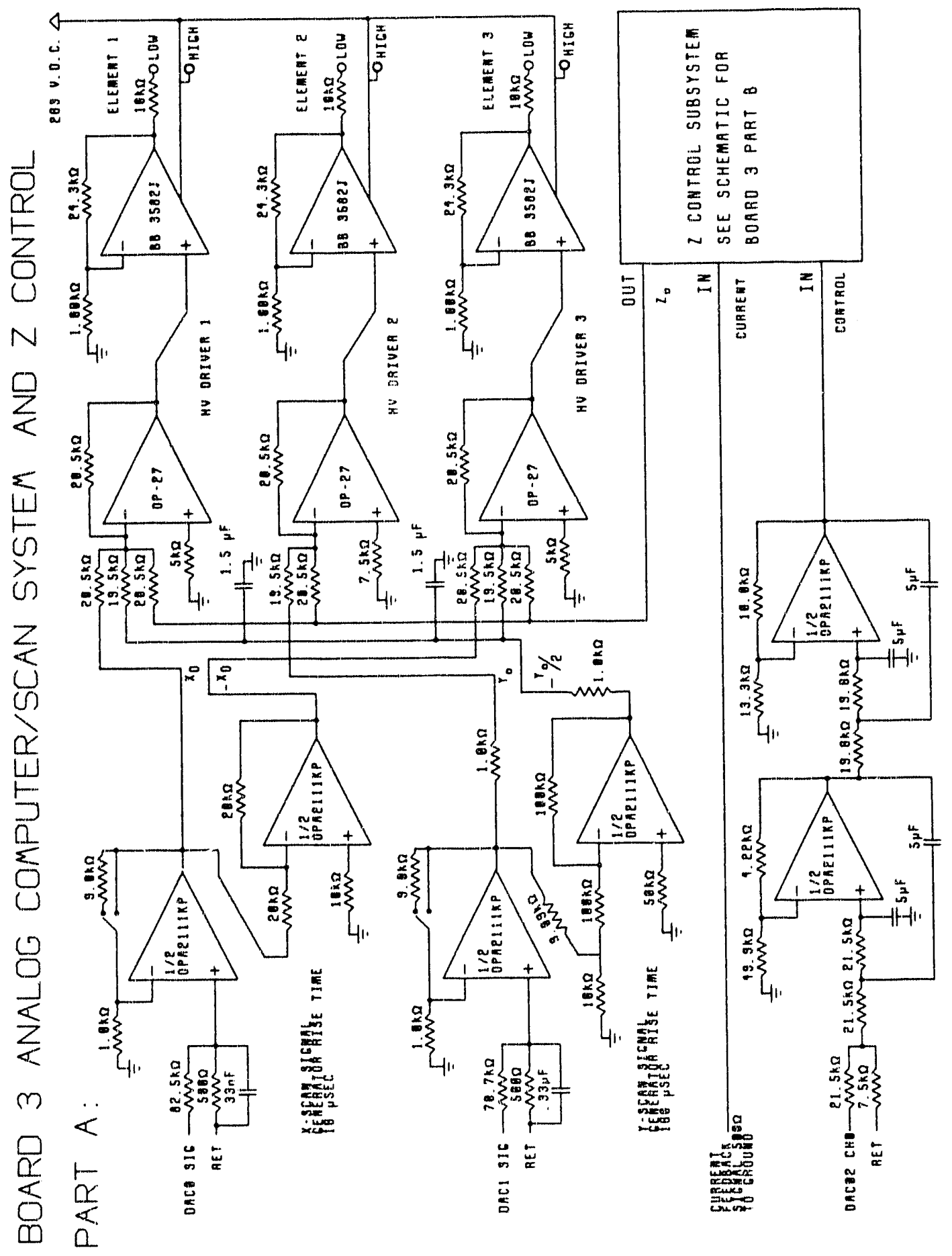


Figure D4: part B of the circuit on board 3. This is the circuitry that is responsible for vertical control via feedback. The tunneling current and control signals are compared with a high-speed comparator. The comparator is powered by voltage regulators to prevent high-apeed oscillations in the supply rails. An integrator follows the comparator, this component is the alow link in the feedback loop to ensure stability. Output from the integrator produces vertical deflections of $10 \mathrm{~nm}$ per volt. A twin-T notch filter centered at $5.8 \mathrm{kHz}(-60 \mathrm{~dB})$ after the integrator counteracts a resonance in the scanning PzT. The comparator/integrator feedback system allows for better loop response speed control while keeping good small signal compensation. simple integrator circuits tend to lose the small aignal response reguired to maintain junction stability when long riso-times are used. The sample/hold amplifier is usually kept in follower mode, allowing transmission of the integrator signal. The amplifier is set in hold mode for operation in fixed height mode. The last amplifier adds the output from the integrator to a constant offset, which places the scanning elements at nominally half-extension, allowing for travel to produce acan deflections. 

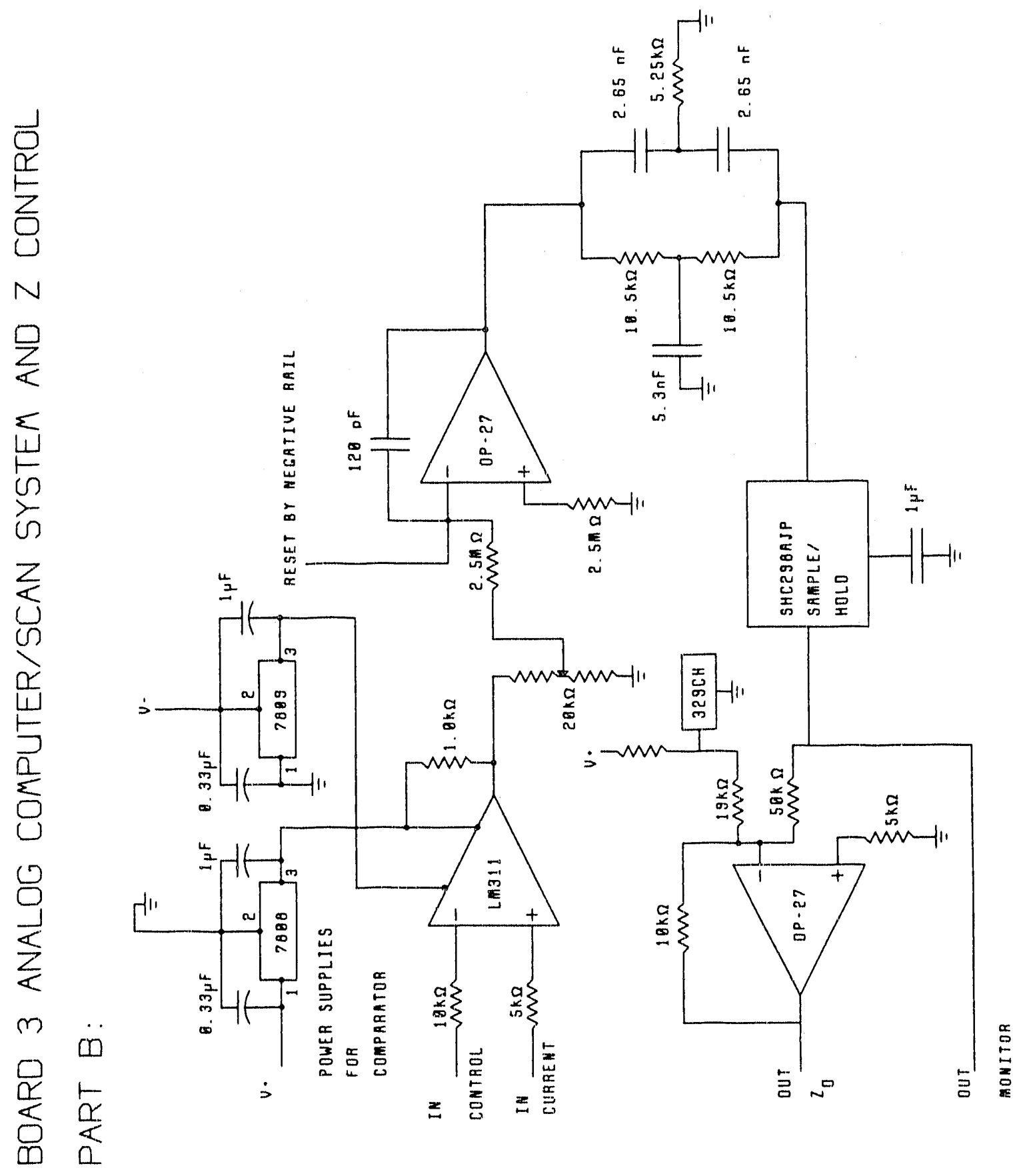
Figure D5: This board houses the current-to-voltage conversion and tunneling current signal processing circuitry. A dual operational amplifier is used for current-to-voltage conversion and buffering the guard line in the eurrent lead. The output from the current-to-voltage converter is passed through an a.c. bandpass filter which allows detection of rapidly changing components of the tunneling current. This can be used for detection of small aignal conductance or current corrugations from fixed height scans. The current-to-voltage converter also sends to a signal to an optional inverter. The optional inverter is used to keep the proper sign of the tunneling current signal going to the feedback circuitry. The output of the optional inverter goes both to the feedback circuitry and external monitors of the $d . c$. current. 


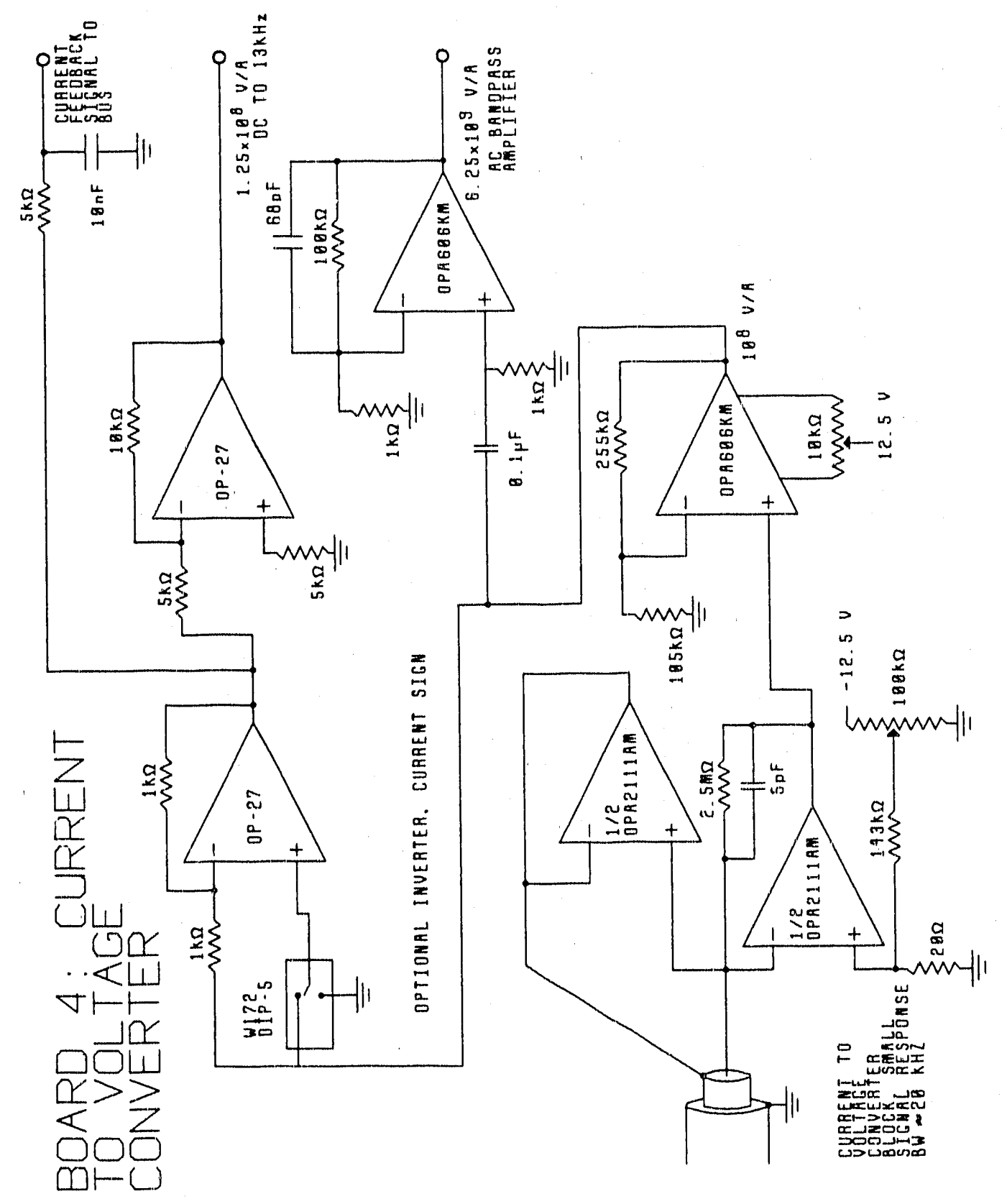



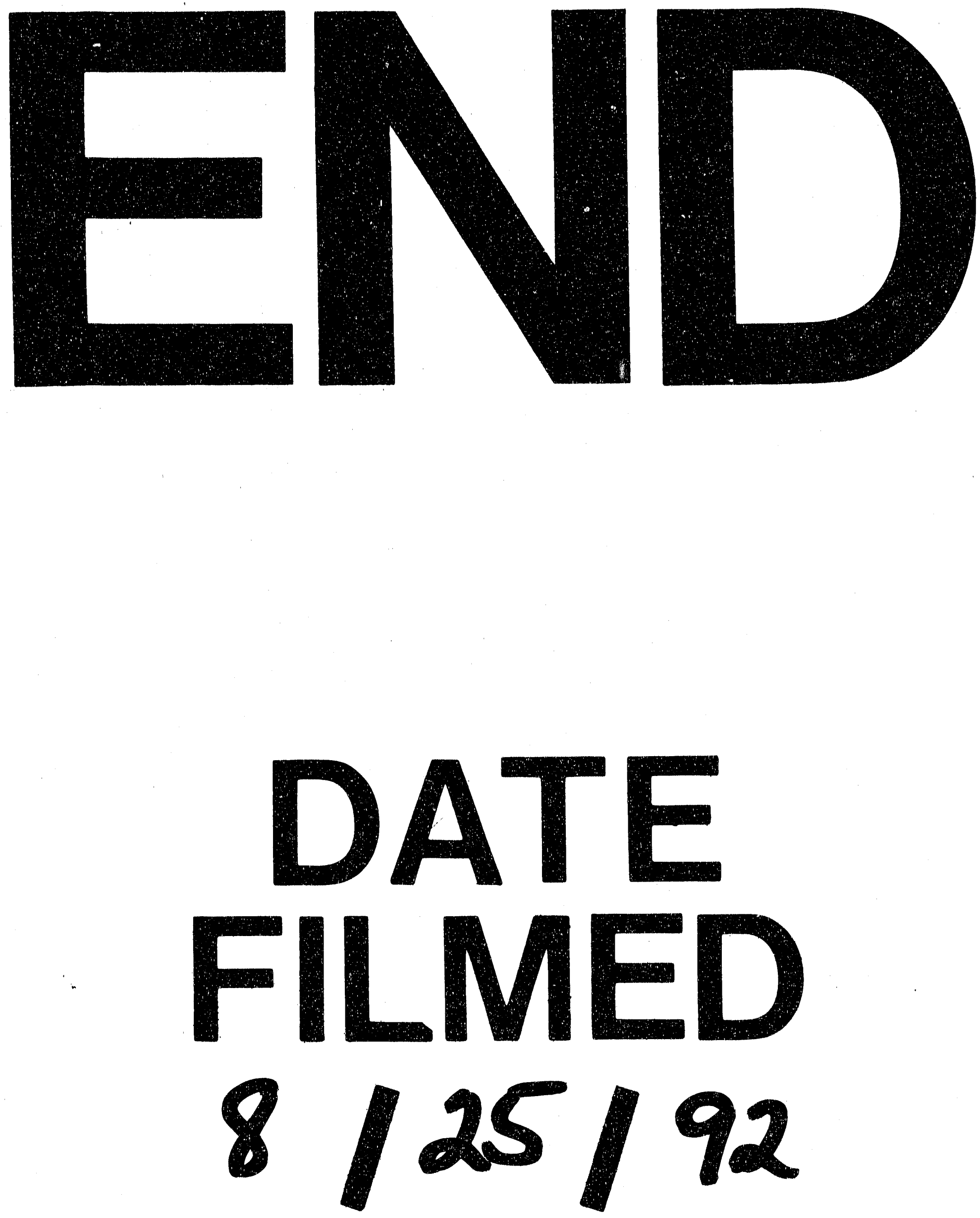
